\title{
PREDICTING UNDERGROUND TUNNEL HAZARDS USING MACHINE LEARNING TECHNIQUES
}

NING LI

Doctor of Philosophy

Universidad Politécnica de Madrid

September 2017 



\section{PREDICTING UNDERGROUND \\ TUNNEL HAZARDS USING MACHINE \\ LEARNING TECHNIQUES}
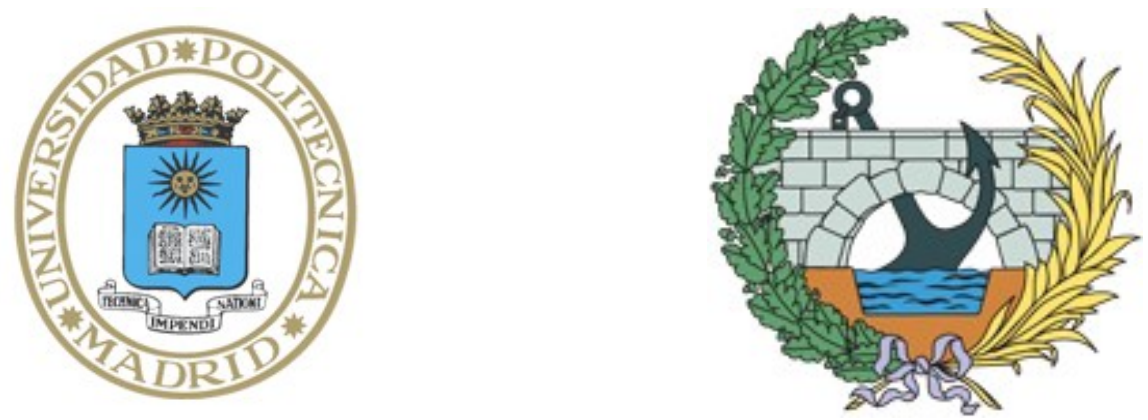

Ning Li

Director: Rafael Jiménez Rodríguez

Laboratorio de Geotechnia

E.T.S.I Caminos, Canales y Puertos

Universidad Politécnica de Madrid

This dissertation is submitted for the degree of Doctor of Philosophy

Madrid, 2017 

Título de la Tesis:

Predicting underground tunnel hazards using machine learning techniques

Autor: Ning Li

Director: Rafael Jiménez Rodríguez

Tribunal nombrado por el Mgfco. y Excmo. Sr. Rector de la Universidad Politécnica de Madrid, el día de de

\section{TRIBUNAL CALIFICADOR}

Presidente:

Vocal $1^{\circ}$ :

Vocal $2^{\circ}$ :

Vocal $3^{\circ}$ :

Secretario:

Realizado el acto de defensa y lectura de la tesis el día de de en Madrid, los miembros del Tribunal acuerdan otorgar la calificación de: 



\section{ABSTRACT}

This dissertation employs and develops machine learning techniques to be used in underground tunnel hazards analyses, with a particular emphasis on (i) the establishment and selection of linear classifier and Bayesian networks models for longterm rock burst prediction; (ii) the development of a Rock Engineering System approach incorporating Artificial Neural Networks (ANN) for short-term rock burst damage estimation; (iii) the comparison of different machine learning algorithms for prediction of rock tunnel squeezing.

(1) For long-term rock burst prediction, we present a linear logistic regression classifier model to predict the occurrence of rock burst probabilistically. Our approach provides estimates of rock burst with simple and easy applicable expressions, which can be employed for risk analyses. For further prediction work, and to cope with incomplete databases, Bayesian networks are presented. We propose and compare three different basic structures, and the Tree augmented Naïve Bayes classifier (TAN) is employed as the optimal structure.

(2) For short-term rock burst prediction, we present an approach based on the Rock Engineering Systems paradigm and on the ANN algorithm, which are used to establish a rock burst damage scale index (RDSI). A threshold of RDSI values is proposed to discriminate the RDSI into three scales. The RDSI can predict the damage scale reliably, hence simplifying the short-term rock burst damage prediction on site. 
(3) For tunnel squeezing prediction, we propose six different machine learning algorithms to train, validate, compare and evaluate three simple models. The joint use of a model based on $Q, H$ and $K$ as input parameters, together with the Random Forests algorithm, is proposed as the optimal solution due to their stability and predictive ability in the training and validation work, hence being a useful tool for prediction of rock tunnel squeezing. 


\section{RESUMEN}

Esta tesis emplea y desarrolla técnicas de aprendizaje automático para ser utilizadas en el análisis de riesgos de túneles subterráneos, con particular énfasis en: (i) el establecimiento y selección de modelos de clasificadores lineales y redes Bayesianas para la predicción del 'estallido de rocas' (rock burst) a largo plazo; ii) el desarrollo de un Sistema de Ingeniería de Rocas (RES, según sus siglas en inglés) que emplee las redes neuronales artificiales (ANN) para la estimación a corto plazo del daño causado por el rock burst; (iii) la comparación de diferentes algoritmos de aprendizaje automático para la predicción del fenómeno fluencia excesiva (squeezing) en túneles construidos en roca.

(1) Para la predicción del rock burst a largo plazo, se presenta un modelo clasificador de regresión logística lineal para predecir su occurencia de forma probabilística. Este enfoque proporciona estimaciones con expresiones simples y fáciles de aplicar, que además pueden emplearse para el análisis de riesgos. En este sentido, las redes Bayesianas se presentan como una metodología apropiada para ser usada en futuros trabajos de predicción, así como también para hacer frente a bases de datos incompletas. Se proponen y se comparan tres diferentes estructuras básicas, y se emplea como estructura óptima el clasificador Bayesiano Tree augmented Nä̈ve Bayes (TAN).

(2) Para la predicción del rock burst a corto plazo, se presenta un enfoque basado en el paradigma de los Sistemas de Ingeniería de Rocas (RES) y en las redes 
neuronales (ANN), que se utilizan para establecer un Índice de Escala de Daños asociados al 'Estallido de Rocas' (Rock burst damage scale index, o RDSI). Se propone un umbral de valores RDSI para discriminar entre tres posibles escalas de daño. El RDSI puede predecir la escala de daños de forma fiable, simplificando así la predicción de daños a corto plazo en el sitio.

(3) Para la predicción del fenómeno del squeezing en túneles, se proponen seis algoritmos de aprendizaje automático diferentes para entrenar, validar, comparar y evaluar tres modelos simples. El uso conjunto de un modelo basado en $Q, H$ y $K$ como parámetros de entrada, junto con el algoritmo de "Bosques Aleatorios" (Random Forests, o RF), se propone como solución óptima, debido a su estabilidad y capacidad predictiva en el trabajo de capacitación y validación, por lo que se convierte en una herramienta útil para predicción del fenómeno squeezing en túneles en roca. 


\section{ACKNOWLEGEMENTS}

I would like to thank my supervisor Rafael Jimenez sincerely, for his instructive guidance and ongoing encouragement of my study and life in Madrid during the past four years. His scientific attitude and perspective enlighten me in the research work with a free and open atmosphere in the laboratory. Without his patient instruction, this dissertation could not been finished.

I would also like to express my great appreciation to the faculty, students, and staff in E.T.S.I Caminos, Canales y Puertos. I would like to thank Prof. Claudio Olalla Marañon, Prof. José María Goicolea and other professors for their enlightened course particularly. Also, I want to thank Angel Alvarez and Valentin Bella for the help of enrollment. Thanks are also due to all the members in Laboratorio de Geotecnia — Dr. Salvador Senent, Prof. Rubén Galindo, Jose Gregorio Gutierrez Chacon, Congke Yi, and other folks - for their help in my research and life. For the doctoral students already graduated in the lab, such as Xianda Feng, Peng Zeng and Miriam Cabrera, I also show gratitude for their patient help in the past years.

Thanks to the China Scholarship Council (CSC) for affording the PhD scholarship and to Fundación José Entrecanales for providing insurance coverage.

I am also grateful to my master supervisor, Prof. Linming Dou, from China University of Mining and Technology, for his constant encouragement and care about my study abroad.

Many good friends, such as Bo Qu, Xidong Wang, Dr. Kunyang Fan and Chao Chang, 
make my life in Madrid unforgettable and colorful. I am very appreciate to their friendship.

Finally, I show my greatest appreciation to my family for their love and support. 


\section{CONTENTS}

ABSTRACT

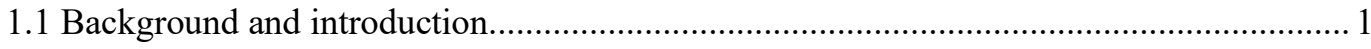

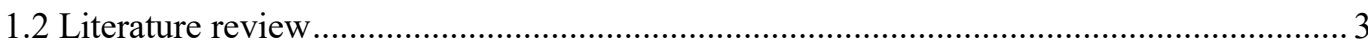

1.2.1 Previous methods to analyze underground tunnel hazards ...................................... 3

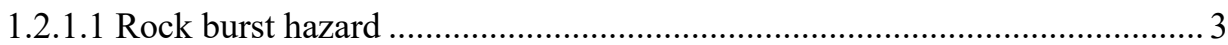

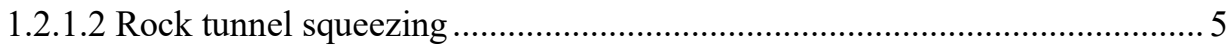

1.2.2 Applications of machine learning techniques ....................................................

1.2.2.1 Introduction to machine learning techniques ............................................. 7

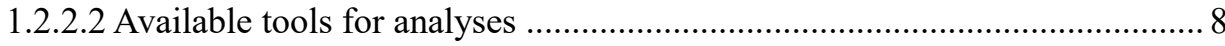

1.2.2.3 Previous applications of machine learning algorithms in the geotechnical

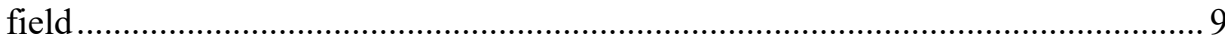

1.2.2.4 Applications in rock burst prediction ......................................................... 9

1.2.2.5 Applications in rock tunnel squeezing prediction ....................................... 10

1.2.3 Limitations and need for improvement.............................................................. 10

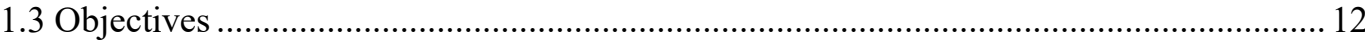

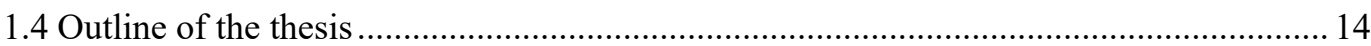

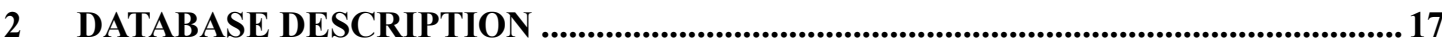

2.1 Database for long-term rock burst prediction .............................................................. 17

2.2 Database for short-term rock burst prediction ............................................................. 21 
2.3 Database for tunnel squeezing prediction............................................................ 24

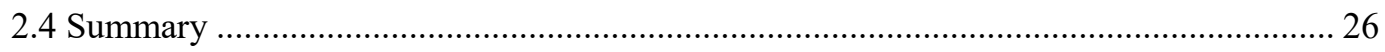

3 A LINEAR CLASSIFIER FOR LONG-TERM PROBABILISTIC PREDICTION OF ROCK BURST HAZARD ............................................................................................. 27

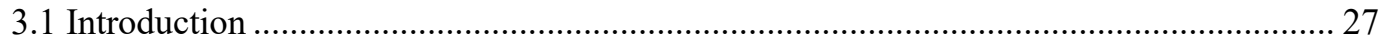

3.2 Parameter selection for long-term rock burst prediction ............................................. 28

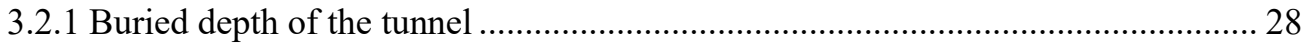

3.2.2 Maximum tangential stress of the surrounding rock ...................................... 28

3.2.3 Uniaxial compressive and tensile strength .................................................. 29

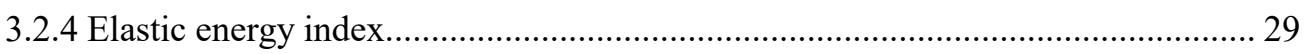

3.2.5 Burst energy release index.................................................................. 30

3.2.6 Duration of dynamic fracture ................................................................ 30

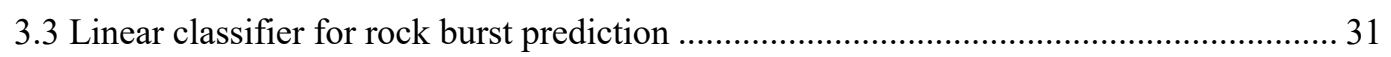

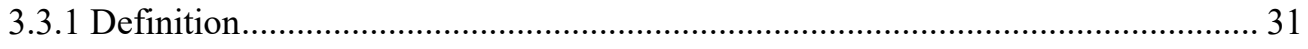

3.3.2 Logistic function..................................................................................... 31

3.3.3 Application in rock burst prediction ...................................................... 32

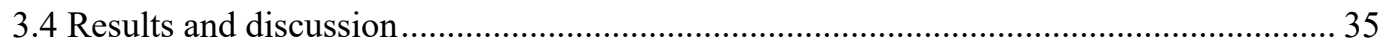

3.4.1 Model training and probability calculation...................................................... 35

3.4.2 Goodness of fit and predictive performance............................................... 41

3.4.2.1 Deviance and Akaike's information criterion ...................................... 41

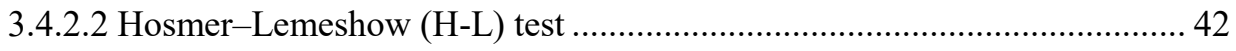

3.4.2.3 Confusion matrices ........................................................................ 43

3.4.2.4 Relative operating characteristic curve .............................................. 44

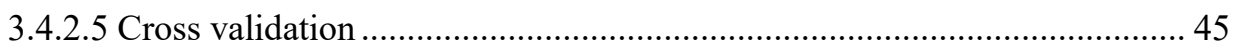

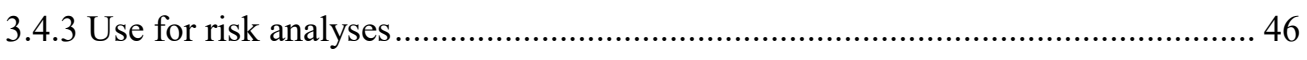

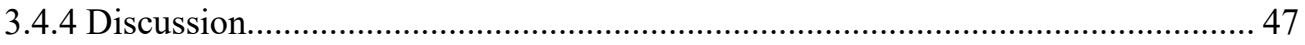

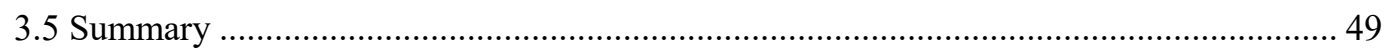

4 PREDICTING ROCK BURST HAZARD WITH INCOMPLETE DATA USING

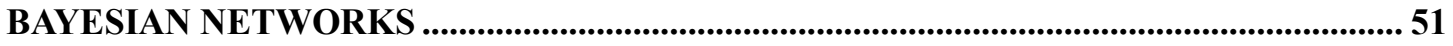

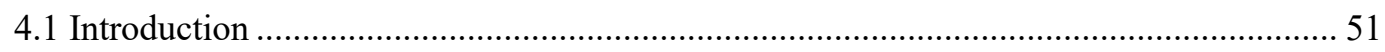

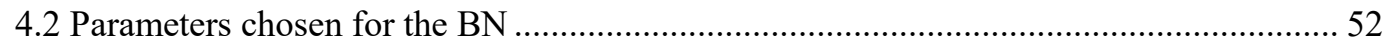

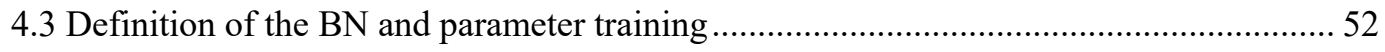




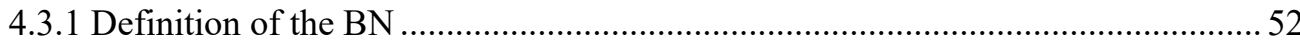

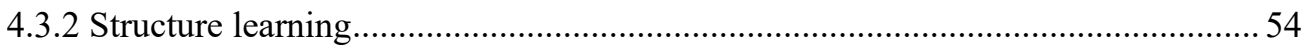

4.3.3 Discretizing the continuous parameters ......................................................... 58

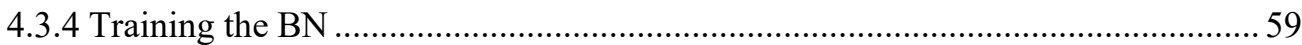

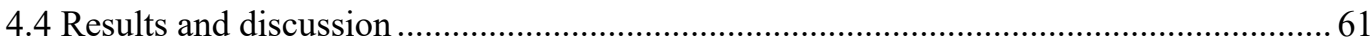

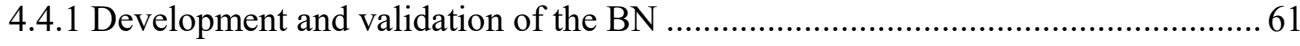

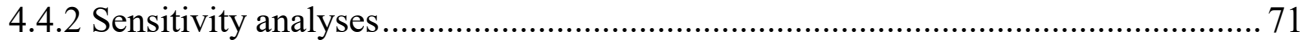

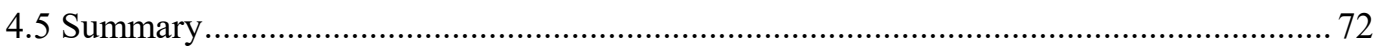

5 EVALUATING SHORT-TERM ROCK BURST DAMAGE USING ROCK ENGINEERING SYSTEMS .........................................................................................75

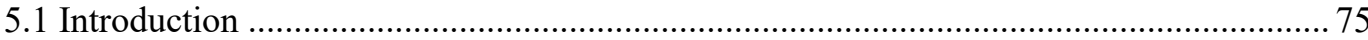

5.2 Description of parameters and of the prediction system.......................................... 76

5.2.1 Description of selected parameters ........................................................ 76

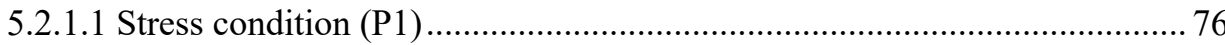

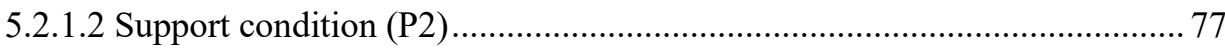

5.2.1.3 Excavation environment (P3) ….......................................................... 77

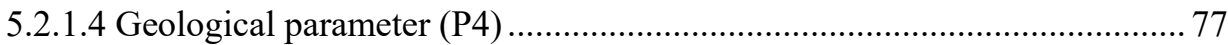

5.2.1.5 Seismic related data (P5 and P7) ...................................................... 78

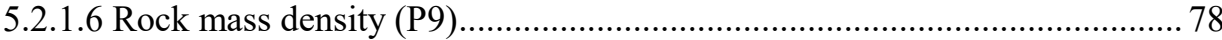

5.2.1.7 Rock burst damages scale (RDS) (P14) ............................................. 79

5.2.2 Rock burst damage prediction system ....................................................... 80

5.3. The Rock Engineering Systems (RES) approach ............................................. 81

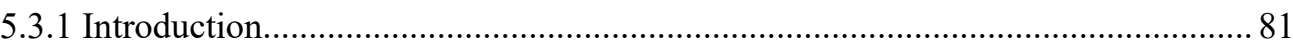

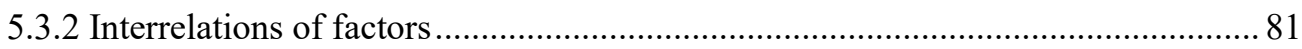

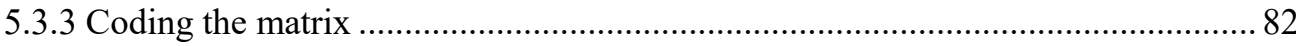

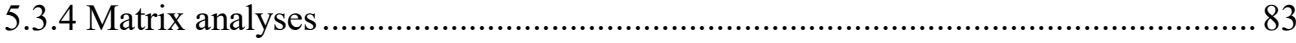

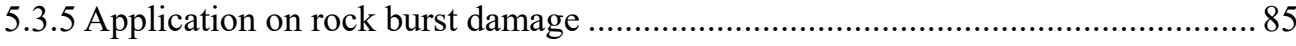

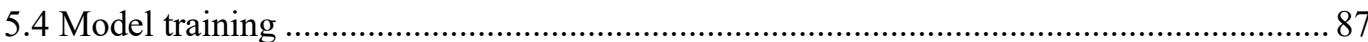

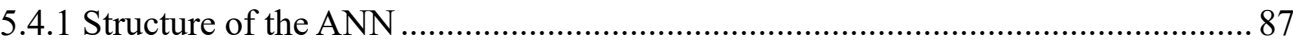

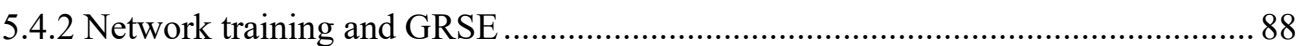

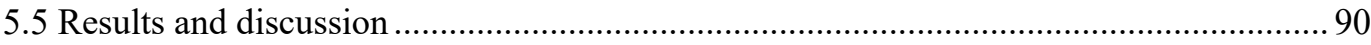

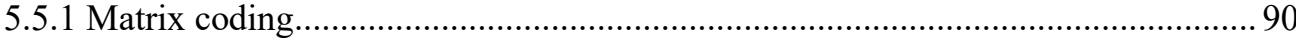


5.5.2 Rock burst damage scale index (RDSI) ....................................................... 92

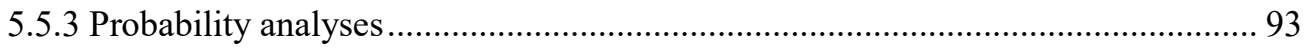

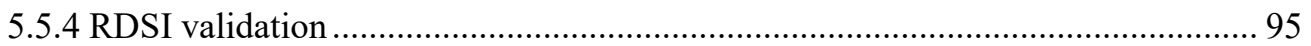

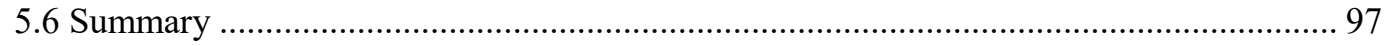

6 PREDICTING TUNNEL SQUEEZING WITH MACHINE LEARNING

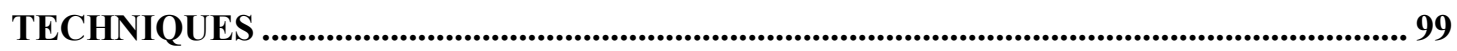

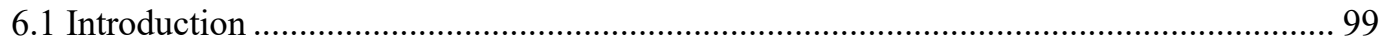

6.2 Selection of parameters and model construction ...................................................... 100

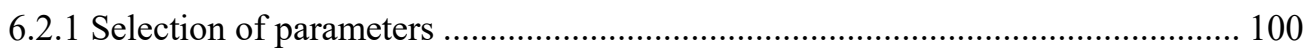

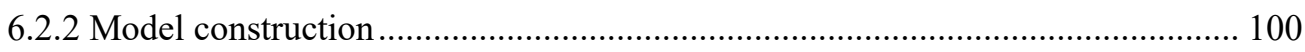

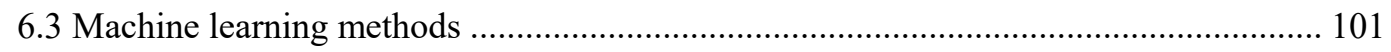

6.3.1 Flowchart of the analyses ................................................................................. 101

6.3.2 Gradient Boosting Machines (GBM) ............................................................. 103

6.3.3 Artificial Neural Networks (ANN) ................................................................... 104

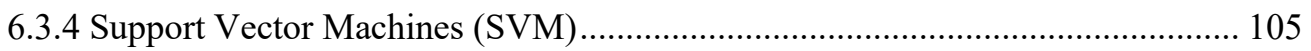

6.3.5 Random Forests (RF),.................................................................................... 106

6.3.6 Multivariable Adaptive Regression Splines (MARS) ......................................... 107

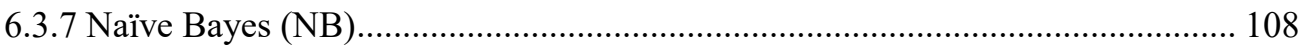

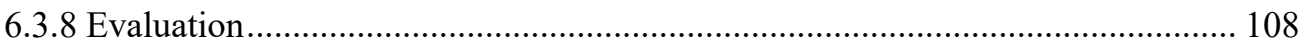

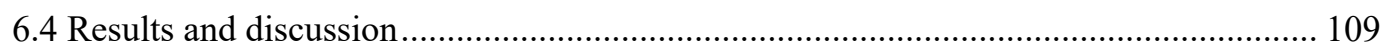

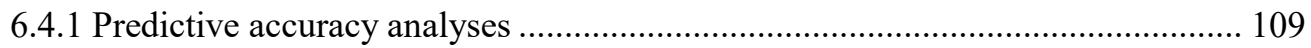

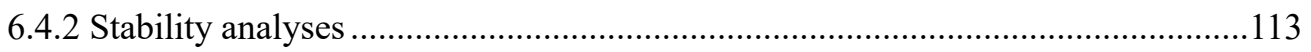

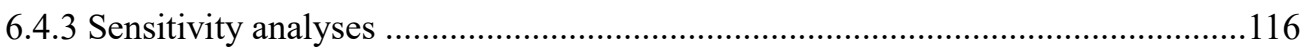

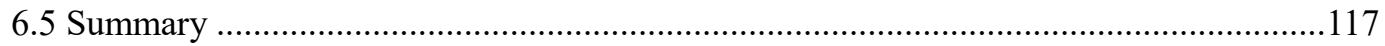

7 CONCLUSIONS AND FUTURE WORK ..............................................................................119

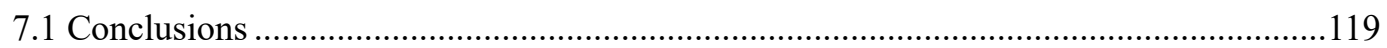

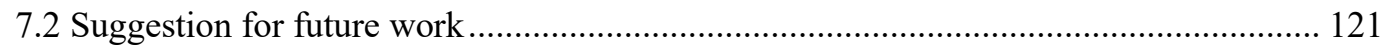

REFERENCES ............................................................................................................................. 123

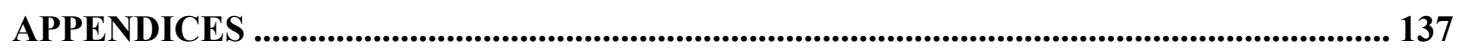

Appendix A Database of 135 case histories of rock burst for long-term prediction.............. 138

Appendix B Database of 254 case histories of rock burst damage scales for short-term 


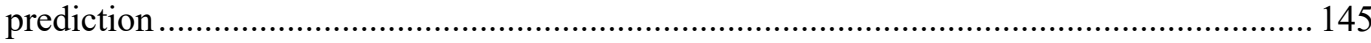

Appendix C Database of 166 case histories of rock tunnel squeezing .................................. 156

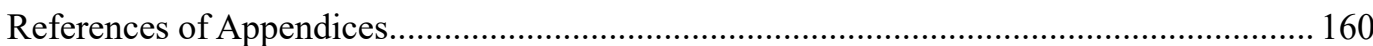





\section{LIST OF TABLES}

Table 1.1 A summary of previous empirical criteria for rock burst prediction ................................. 4

Table 1.2 A summary of previous empirical criteria for rock tunnel squeezing prediction............... 7

Table 2.1 Parameters in the database for long-term rock burst prediction...................................... 18

Table 2.2 Descriptive statistics of the input parameters for case histories within the long-term rock burst database

Table 2.3 Pearson Correlation coefficients (p-values) between the parameters collected in the rock burst database (The p-value is expressed in probability levels so that smaller p-levels indicate a more significant relationship)

Table 2.4 Parameters descriptions, types and selection 22

Table 2.5 Descriptive statistics of the input parameters for case histories within the short-term rock burst database

Table 2.6 Descriptive statistics of the input parameters for case histories within the squeezing database

Table 3.1 Possible models using parameters collected

Table 3.2 Iterative parameter estimates for the linear classifier Model B. .38

Table 3.3 Summary of deviances and AIC for Models A and B

Table 3.4 The H-L test for Model A.

Table 3.5 The H-L test for Model B.

Table 3.6 Confusion matrix of "Rock burst" prediction with Model A .............................................4 44

Table 3.7 Confusion matrix of "Rock burst" prediction with Model B .............................................4

Table 3.8 9-fold cross-validation results of Model B

Table 3.9 Comparison of error rates with four different empirical methods and with the linear classifier of Model B with all data and with cross-validation.

Table 4.1 Summary of the intervals applied to the input parameters of the BN 59

Table 4.2 Confusion matrices of node "Rock burst" using three different classifiers 63

Table 4.3 Comparison of structures and influence on the predictive performance........ 64 


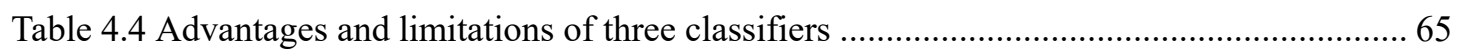

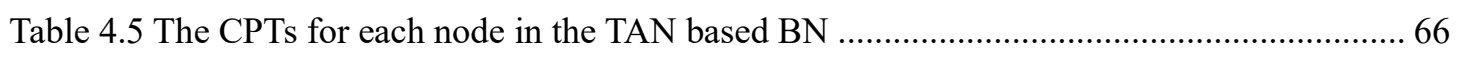

Table 4.6 Comparison of Accuracy values with four different methods and our BNs ................... 67

Table 4.7 The confusion matrices of the 8 groups of data employed by the cross-validation (The

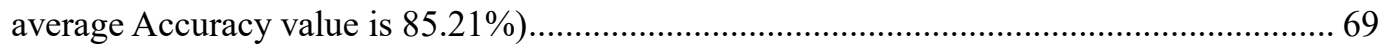

Table 4.8 Results of validation with new rock burst case histories ............................................... 70

Table 4.9 Results of sensitivity analyses for node "Rock burst" in the BN model......................... 72

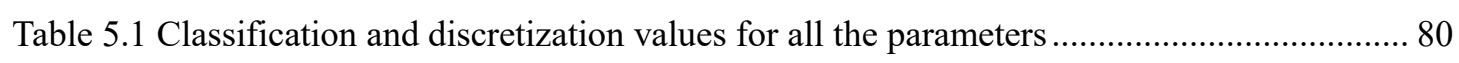

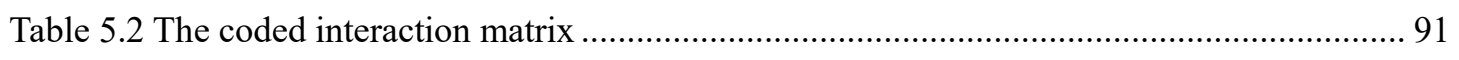

Table 5.3 Weight of each parameter for the rock burst damage prediction .................................... 92

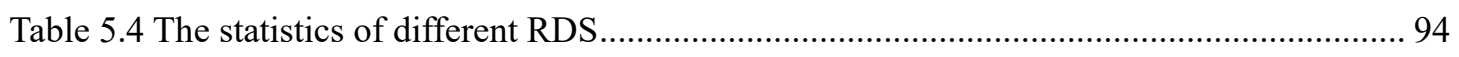

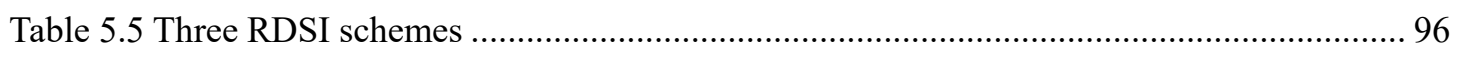

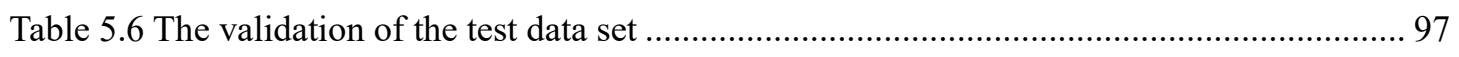

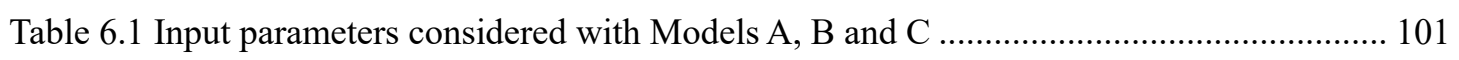

Table 6.2 Tuning parameters, values and optimal parameters for each model............................. 109

Table 6.3 Results of the training performance with different machine learning methods for the

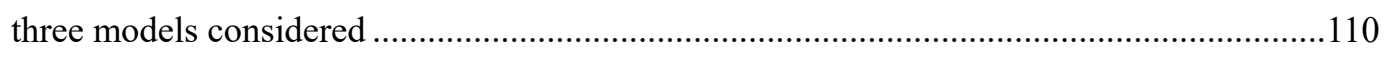

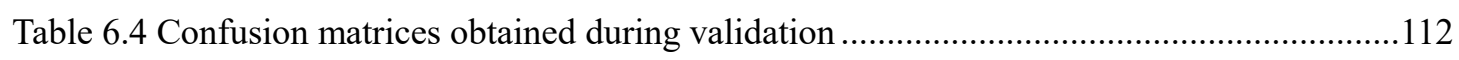

Table 6.5 Results of the validation performance with different machine learning methods for the three models considered.

Table 6.6 Performance coordinates and ranks of different machine learning techniques for tunnel squeezing prediction

Table 6.7 Results of the parameter importance or sensitivity analyses using the algorithm with three predictive models. 


\section{LIST OF FIGURES}

Figure 2.1 Boxplot of parameters employed for rock burst prediction ('N' represents no rock burst and ' $\mathrm{Y}$ ' represents rock burst)

Figure 2.2 Histograms of the five parameters considered to predict the rock burst hazard .21

Figure 2.3 Boxplot of all the parameters for different levels of damage classification (R2 to R5) 23

Figure 2.4 Boxplot of parameters selected for tunnel squeezing predction ("N" indicates case histories with no squeezing, and "Y" indicates squeezing) ..................................................25

Figure 2.5 Interaction matrix of the variables in the tunnel squeezing database ............................26

Figure 3.1 The single loading-unloading cycle of uniaxial compression and definition of $\mathrm{W}_{\mathrm{et}} \ldots . .30$

Figure 3.2 The logistic function (Sigmoid function) ……............................................................. 32

Figure 3.3 Equi-probability lines for rock burst prediction with Model A ..................................... 40

Figure $3.450 \%$ of probability plane for rock burst prediction with Model B .................................40

Figure 3.5 Equi-probability planes (viewed under a certain angle) for rock burst prediction with

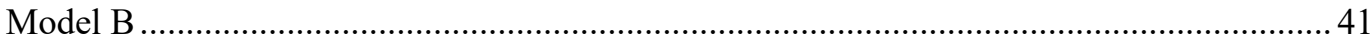

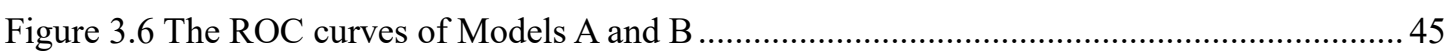

Figure 4.1 A simple Bayesian network with six parameters (based on Pearl (1985)).....................53

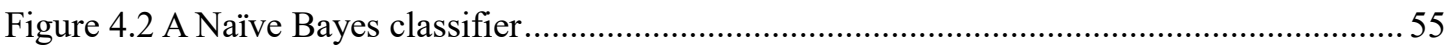

Figure 4.3 A Tree augmented Nä̈ve Bayes classifier .....................................................................56

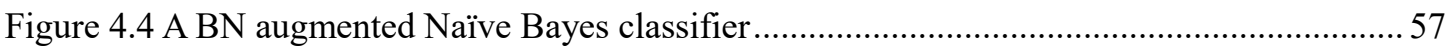

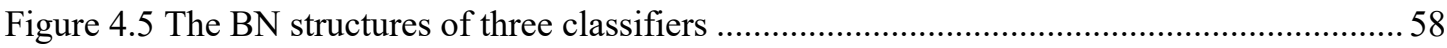

Figure 4.6 The BN after the EM algorithm is applied to the input parameters for training,

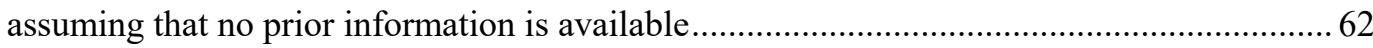

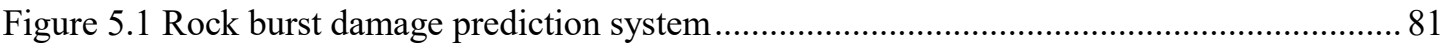

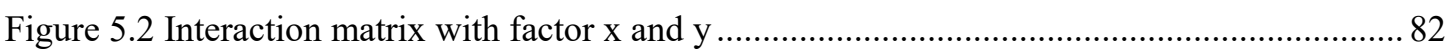

Figure 5.3 A $2 \times 2$ interaction matrix with leading diagonal terms, rock discontinuity and rock stress

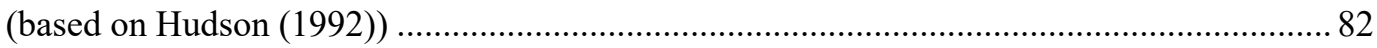

Figure 5.4 Interaction matrix and related C and E (based on Hudson (1992)) ..............................84 
Figure 5.5 The (C, E) plots with $m$ influencing factors (based on Hudson (1992))

Figure 5.6 The (C, E) plot with lines of equal parameter interaction intensity and dominance (based on Hudson (1992))

Figure 5.7 The RES matrix for RDS prediction 86

Figure 5.8 The structure of common ANN.

Figure 5.9 The cause and effect for the selected parameters

Figure 5.10 Distribution of RDSI (from R2 to R5): values for different levels of rock burst damage, when low different damage scales are considered

Figure 5.11 Distribution of RDSI (from R2\&3 to R5): values for different levels of rock burst damage, when low different damage scales are not considered and combined.....

Figure 5.12 PDF of the three damage scales

Figure 5.13 Curves of scales likelihood of rock burst damage for a given RDSI

Figure 6.1 Flowchart of the procedure for squeezing prediction in rock tunnels using different machine learning techniques

Figure 6.2 An example of a separable problem in a two dimensional space from Cortes and Vapnik (1995).

Figure 6.3 Boxplot distribution of results obtained during the train process (Accuracy and Kappa metrics with different machine learning techniques).

Figure 6.4 3D distribution of relative performances, with different algorithms, during training and validation (For instance, the point named GBM represents the training set performance and GBM' represents the performance with the validation data set) 114

Figure 6.5 2D distribution of relative performances with different algorithms. 115 


\section{LIST OF APPENDICES}

Appendix A Database of 135 case histories of rock burst for long-term prediction 138

Appendix B Database of 254 case histories of rock burst damage scales for short-term prediction 145

Appendix C Database of 166 case histories of rock tunnel squeezing 156 



\section{INTRODUCTION}

\subsection{Background and introduction}

Rock burst and tunnel squeezing are two frequent difficulties encountered during construction of tunnels and other underground facilities. Rock burst is a dynamic process caused by a sudden and violent release of elastic energy accumulated in rock and coal masses which, given its 'explosive' nature, can lead to significant risks. The reason is that it produces ejection of rock fragments, which could lead to casualties, to failure and deformation of the supporting structures, and to damage of equipment (see e.g. Brauner 1994; Ortlepp and Stacey 1994; Kaiser et al. 1996; Huang and Wang 1999; Dou et al. 2012). Its economic consequences in the civil and mining engineering sectors are significant. For instance, it can impose a threat on human of life, as demonstrated by more than 13000 accidents, with more than 16000 casualties, that have been reported to have occurred in metal mining in China between 2001 and 2007 (Zhou et al. 2012). And it can also affect the schedule and budget of projects, as illustrated by the 
experience of the two Jinping-II hydropower tunnels, where the cumulative lengths of sections with rock burst occurrence added to (approx.) $18.5 \%$ and 16.3\% (Shan and Yan 2010). Rock burst problems are also common in coal mining. For instance, a serious rock burst accident occurred in Sunjiawan coal mine in Fuxin (Liaoning province; February 14, 2005), leading to a significant seismic event with Richter magnitude of 2.5 associated to a large gas emission (Dou et al. 2009); or in Qianqiu coal mine in Henan province (November 3, 2011), with 10 people killed and 75 trapped underground.

Tunnel squeezing, on the other hand, refers to the time-dependent (and large) deformations that typically occur due to plastic flow in tunnels constructed in weak ground, as rock squeezing is essentially associated with creep caused by stresses exceeding a limiting shear strength (Barla 1995). The negative consequences of tunnel squeezing have been repeatedly reported since it was identified for the first time during construction of the Simplon Tunnel in Switzerland (Yassaghi and Salari-Rad 2005), and they often require mitigation measures that range from special excavation and support measures (Schubert 1996; Dalgıç 2002); to reexcavation of sections, or even to reconstruction of failed supports (Barla and Pelizza 2000; Hoek and Guevara 2009; Gao et al. 2015).

Being able to anticipate the likelihood of such hazards, and their associated consequences, is considered as an important (and challenging) topic for research. This thesis is an attempt to contribute in that direction. Therefore, we first review traditional methods for prediction of these two underground tunnel hazards - rock burst and squeezing - and then we attempt to further develop methods to predict those hazards using machine learning techniques, using databases collected for such task. 


\subsection{Literature review}

This section reviews the development of analytical and empirical methods to predict rock burst and rock tunnel squeezing hazards, and their contributions, applicability and limitations are discussed. And, to serve as a foundation of the future discussion, the machine learning techniques that will be employed in the following chapters of this thesis are also introduced.

\subsubsection{Previous methods to analyze underground tunnel hazards}

\subsubsection{Rock burst hazard}

Predicting the (likely) occurrence of rock burst in one specific project using limited information is one of main challenges that still exists in relation to rock burst, as the complexity of the phenomena involved has made it difficult to predict rock burst in real cases. The prediction of rock burst can be divided into two categories: long-term and short-term predictions (Peng et al. 2010). Long-term prediction aims to assess preliminarily, based on simple information that is commonly available during the initial stages of a project, the likelihood of rock burst occurring during the future development of the project; the objective is that such prediction can serve as guidance for decision making in relation to excavation and control methods. Short-term prediction aims to predict the location, the time, and the damage level of rock burst occurrence based on data — such as information about drilling bits, micro seismic monitoring, and acoustic emission - collected during construction of the actual underground facility.

Several theories — such as the 'strength theory', the 'rigidity theory' and the 'energy theory', etc. - were proposed since the 1950's to explain the mechanism leading to rock burst occurrence. After that, new 'burst liability' theories that employ the elastic 
energy index, the burst energy release index and the duration of dynamic fracture to predict rock burst were developed (Dou et al. 2006). Significant advances in the understanding of the processes leading to rock burst have been achieved since the seminal work of Cook (1966). He proposed a theoretical method to predict rock burst, considering that rock burst occurs as a result of the excessive energy during the deformation stage of rock. Then, Zubelewicz and Mroz (1983) simulated the dynamic instability of rock burst, and Xie and Pariseau (1993) used fractal geometry and chaos theory to study the rock burst mechanism. Prevention and control methods for rock burst hazard related to such mechanisms were also developed. For instance, Kaiser et al. (1996) recommended supports against rock burst based on the energy release, and Cai (2013) proposed principles of rock support design under burst-prone ground condition.

In addition, many simple criteria have also been proposed to predict long-term rock burst (see Table 1.1 for a summary of the most common ones). It can be noted that these empirical methods only employ 2 or 3 parameters that are available at the initial stage of the projects, so that they can be applied easily on site.

Table 1.1 A summary of previous empirical criteria for rock burst prediction

\begin{tabular}{ll}
\hline Criteria & References \\
\hline$H_{c r}=0.318 \sigma_{c}(1-\mu) /(3-4 \mu) \gamma$ & Hou and Wang (1989) \\
$H_{c r}=\sigma_{c}(1-\sin \varphi) \lambda\left[-1+(1+E / \lambda)^{l /(1-\sin \varphi)}-E / \lambda\right] / 2 E \lambda \sin \varphi$ & Pan and Li (2002), Pan and Li \\
& $(2005)$ \\
$W_{e t} \geq 2.0$ & Kidybiński (1981), Wang et al. \\
$\sigma_{\theta} / \sigma_{c} \geq 0.3$ & $(1998)$ \\
$\sigma_{c} / \sigma_{t} \leq 40$ & Wang et al. (1998) \\
$\sigma_{\theta} / \sigma_{c}>0.2$ & Wang et al. (1998) \\
\hline
\end{tabular}

Notation: $\sigma_{\theta}$ is the maximum tangential stress of surrounding rock, MPa; $\sigma_{c}$ is the uniaxial compressive strength of rock, $\mathrm{MPa} ; \sigma_{t}$ is the uniaxial tensile strength of rock, $\mathrm{MPa} ; \mu$ is the Poisson's ratio; $\gamma$ is the weight of the rock mass; $\varphi$ is the internal friction angle of rock; $\lambda$ is the softening modulus (value of elastic modulus after the peak value of stress in the stress-strain curve), MPa; E is the elastic modulus, MPa; $W_{e t}$ is elastic energy index. 
With the development of dynamic systems to record data on site continuously — such as micro seismic, electromagnetic and acoustic emission monitoring devices in underground projects (Cai et al. 2016a; Li et al. 2016)—, large databases of ground condition and of its response during rock burst events were collected, which could be used to analyze short-term rock burst. For instance, a new criterion to assess rock burst hazard using seismic data in coal mines was proposed by Mutke et al. (2015), whereas Zhang et al. (2013) proposed a method for hazard assessment based on seismic energy distribution. Micro seismic precursory characteristics were studied by Cao et al. (2016), and a new geophysical technique, the passive seismic velocity tomography, was applied by Cao et al. (2015) to assess the hazard. Similarly, Lu et al. (2015) analyzed the relationship between the characteristic of the micro seismic data and the actual rock burst events. A theoretical formula between acoustic emission parameters and rock burst occurrence was studied by Zhang et al. (2014). The data of micro seismic and electromagnetic radiation were integrated by $\mathrm{Li}$ et al. (2016) to monitor the rock burst hazard. And a multi-agent information fusion that combined micro seismic, electromagnetic and acoustic data on site for short-term prediction of rock burst has been proposed by Jia et al. (2015).

\subsubsection{Rock tunnel squeezing}

Given its importance in practice, many researchers have attempted to develop methods to predict the hazard associated to rock tunnel squeezing. Fritz (1984) presented an analytical solution for axisymmetric tunnels in elasto-viscoplastic media; whereas Sulem et al. (1987) proposed another solution to characterize tunnel convergences that considered the joint influence of face advance and time-dependent rock mass behavior. Other scholars employed numerical models with advanced time-dependent constitutive 
laws: Debernardi and Barla (2009) employed a stress-hardening elastic-viscous-plastic model with a new viscoplastic constitutive law to incorporate the most important features of convergence behavior observed in tunnels, and a visco-elastio-plastic model considering the visco-elastic (primary) and visco-plastic (secondary) contributions to rock creep was proposed by Sterpi and Gioda (2007). Similarly, Gioda and Cividini (1996) and Shalabi (2005) conducted finite element analyses (FEM) of the time dependent effects that develop when a tunnel is driven in a rock mass with viscous behavior; whereas Zhu et al. (2003) used FEM analyses to analyze tunnel supports under squeezing conditions. Similarly, Gao et al. (2015) employed UDEC to simulate squeezing due to face advance induced stress in a coal mine tunnel.

Such complex analytical and numerical methods have made empirical methods which aim to anticipate squeezing using previous experiences and relatively simple information that is often available at early design stages — to play an important role to predict squeezing in practice (see e.g., Jethwa et al. 1984; Singh et al. 1992; Barla 1995; Goel et al. 1995; Singh et al. 1997; Hoek 2001; Shrestha 2006; Jimenez and Recio 2011; Dwivedi et al. 2013). Some expressions that are commonly used for such task in practice are listed in Table 1.2. 
Table 1.2 A summary of previous empirical criteria for rock tunnel squeezing prediction

\begin{tabular}{ll}
\hline Criteria & References \\
\hline$N_{c}=\sigma_{c m} / \gamma H \leq 2.0$ with $\sigma_{c m}=2 c_{p} \cos \phi_{p} /\left(1-\sin \phi_{p}\right)$ & Jethwa et al. (1984) \\
$H \geq 270 N^{0.33} \cdot B^{-0.1}$ with $N=(Q)_{S R F=1}$ & Goel et al. (1995) \\
$\sigma_{c m} / \gamma H \leq 1.0$ & Barla (1995) \\
$\sigma_{c m} / p_{0}=\sigma_{c m} / \gamma H \leq 0.35$ & Hoek (2001) \\
$H \geq 350 Q^{1 / 3}$ & Singh et al. (1992) \\
$H \geq 424.4 Q^{0.32}$ & Jimenez and Recio (2011) \\
$\varepsilon=\left(0.0191 \sigma_{v} Q^{-0.2}\right) /(K+1)+0.0025 \geq 1 \%$ with $\sigma_{v}$ & \\
$=0.027 H$ & Dwivedi et al. (2013) \\
\hline
\end{tabular}

Notation: $N_{c}($ or $\alpha)$ is the competency factor (also called "strength stress ratio $(S S R)$ "); $\sigma_{c m}$ is the rock mass uniaxial compressive strength, MPa; $\gamma$ is the rock mass specific weight, $\mathrm{MN} / \mathrm{m}^{3} ; H$ is the overburden or depth of tunnel, $\mathrm{m} ; c_{p}$ is the rock mass peak cohesion, MPa; $\phi_{p}$ is the rock mass peak friction angle, degree; $Q$ is Rock tunneling quality index; $N$ is the rock mass number (or stress-free $Q) ; S R F$ is the stress reduction factor; $B$ is the tunnel span or diameter, $\mathrm{m} ; \varepsilon$ is the percentage strain (ratio of tunnel closure to tunnel diameter); $p_{0}$ is the in situ vertical stress at tunnel depth, MPa; $\sigma_{v}$ is the vertical in situ stress, MPa; $K$ is the support stiffness, MPa.

\subsubsection{Applications of machine learning techniques}

\subsubsection{Introduction to machine learning techniques}

Two broad families of models - generative and discriminative - are commonly employed in machine learning applications. Their difference can be explained in the context of the theorem proposed by Bayes and Price (1763) which can be employed to obtain information about one parameter, $x$, after an additional observation, $y$, becomes available. It can be expressed as:

$$
P(x \mid y)=\frac{P(y \mid x) P(x)}{P(y)}
$$

Generative models are a class of models for learning the joint probability, $P(x, y)$, (with $x$ being the inputs and $y$ the output) in which Bayes rules are used to calculate $P(y \mid x)$, and then to pick the most likely output of $y$ (Ng and Jordan 2002). Popular models among this type of models are the Naïve Bayes algorithm (NB), Bayesian Networks 
(BNs) or Hidden Markov models (HMM).

Discriminative models, also called conditional models, are a class of models in which the conditional probability distribution $P(y \mid x)$ is 'learnt'; in other words, they produce a direct map from inputs $x$ to the class labels $y$ (Ng and Jordan 2002). Popular models among them are Logistic Regressions (LR), Support Vector Machines (SVM), Random Forests (RF) or Artificial Neural Networks (ANN).

Discriminative models can yield a superior performance for classification and regression tasks, as they impose no requirements of the joint distribution to be employed. On the other hand, generative models are typically more flexible to express dependencies in complex learning tasks (Ng and Jordan 2002; Singla and Domingos 2005).

\subsubsection{Available tools for analyses}

There are many free and open-source software packages that provide implementations of a variety of machine learning algorithms. WEKA is a workbench (Holmes et al. 1994) that contains a collection of visualization tools and algorithms for data analyses and predictive modeling, together with graphical user interfaces for easy access to these functions. It is written in Java and released under the GPL. There are three graphical user interfaces ("Explorer", "Experimenter" and "KnowledgeFlow”) as well as a standardized command line interface (Hornik et al. 2009). Another popular open-source software is R. It is a software environment for statistical computing and graphics that is supported by the R Foundation for Statistical Computing (Hornik 2012). The R language is widely used among statisticians and data analysts, and many user-created packages (available at the Comprehensive R Archive Network (CRAN)) of machine learning techniques can be installed and programed for further use. In Chapter 6, the 
performance of several popular machine learning techniques are compared within the $\mathrm{R}$ environment, when they are used to predict hazards associated to geotechnical problems.

There are other software packages like Shogun (Sonnenburg et al. 2010), MLPACK (Curtin et al. 2013), TensorFlow (Abadi et al. 2016) and MLlib (Meng et al. 2016) that can also be employed for machine learning analyses. However, they are not used in this dissertation and, for that reason, they are not further reviewed herein.

1.2.2.3 Previous applications of machine learning algorithms in the geotechnical field Machine learning (ML) methods have been successfully applied to complex problems in geotechnical engineering. The quality of a slate mine was estimated by Matías et al. (2004) using gradient boosting combined with SVM; landslide susceptibility was studied by Micheletti et al. (2014) using AdaBoost (a previous form of Gradient boosting machines (GBM)) combined with SVM and RF; Yang and Zhang (1998) and Naghadehi et al. (2013) combined ANN with the Rock Engineering Systems approach to assess the stability of underground excavations and of slopes in open-pit mining; the blasting vibration characteristic parameters were predicted using RF (Dong et al. 2011); and the RF algorithm was also employed to estimate ground settlements induced by tunnel construction (Zhou et al. 2017); Adoko et al. (2013a) employed Multivariable Adaptive Regression Splines (MRAS) and ANN to predict the tunnel convergence; Zhang and Goh (2016) predicted pile drivability using MARS; and Liu and Cheng (2016) analyzed soil slopes using MARS-based Monte Carlo simulation.

\subsubsection{Applications in rock burst prediction}

Machine learning methods have often been applied for rock burst prediction since the 
seminal work of Feng and Wang (1994) using ANN. For instance, Zhang et al. (2011) employed a Particle Swarm Optimization-BP Neural Network; Zhou et al. (2012) and Peng et al. (2014) proposed a rock burst classification based on SVM; Li and Liu (2015) employed the RF approach; Liu et al. (2013) employed cloud models with attribution weight; and Zhou et al. (2016b) employed stochastic gradient boosting for predicting rock burst damage in burst-prone mines. Others have employed fuzzy technologies (see e.g., Liu et al. (2008), Guo and Jiang (2009), Yu et al. (2009) and Adoko et al. (2013a)) to infer rock burst and its risks; and Bai et al. (2009) developed a Fisher discriminant analysis model (FDA) for rock burst prediction in deep rock engineering.

\subsubsection{Applications in rock tunnel squeezing prediction}

Machine learning could also be useful to address the rock tunnel squeezing problem. As an example, Jimenez and Recio (2011) and Shafiei et al. (2012) employed different classifiers — in particular, a linear classifier and SVM — to predict rock squeezing based on tunnel depth and rock quality; whereas Feng and Jimenez (2015) explored the use of BNs - an efficient and powerful technique, that allows the user to make predictions even in the presence of incomplete data - for the same task.

\subsubsection{Limitations and need for improvement}

The predictive methods discussed above have some limitations in relation to the prediction of the two challenges addressed in this thesis - rock burst and tunnel squeezing-, which makes it worthwhile to attempt new ways to tackle such problems.

For instance, one shortcoming associated to the analytical and numerical methods discussed above is that, although they have been often shown to have "backward" predictive capabilities (i.e., they can be fitted to reproduce the occurrence of an 
observed behavior), their "forward" predictive capabilities have not yet been validated so well, due to the difficulty to assess or fit the model parameters at an early stage of the project, when enough good quality data or performance observations are not yet available. In addition, the complexity of the models involved makes their use to often be beyond the capabilities of many design offices (Hoek and Marinos 2009). Therefore, there is a need for better empirical models that can be used with the limited amount of data that is typically available at the early stages of one project.

Similarly, many of the methods discussed above (and, in particular, the empirical methods listed in Tables 1.1 and 1.2) have the limitation that they are deterministic, hence providing little information about the uncertainties associated to their predictions. In other words, they cannot be used in the context of risk analyses. Therefore, there is a need to develop new methods that probabilistically estimate the likely occurrence (or not) of the rock burst and squeezing problems discussed herein. And, similarly, those traditional methods for rock burst and squeezing predictions are meant to be employed under a 'complete state of information', or when information about all the input parameters that 'feed' the model is available. Since that is often not the case when projects are at an early stage of development, there is a need to develop new methods that can still make prediction under incomplete states of information, being able to update (and, hopefully, improve) such predictions as more information becomes available.

Similarly, and because we are using empirical methods, whose predictive capabilities significantly depend on the quality of the data employed for their development, there is a need to develop even more extensive data sets of good quality case histories associated to the problems under analyses. In that sense, we have made a significant effort to 
further extend the existing databases (e.g., for squeezing prediction) and, to build new ones (e.g., for rock burst prediction), so that they can be employed for the analyses conducted herein and, in future analyses conducted by other researchers. (Note that the databases have been made freely available to other researchers, so that they can use them in their work.)

Finally, it could be noted that machine learning methods overcome some (or many) of the limitations discussed above. However, as shown in Sections 1.2.2.4 and 1.2.2.5, their use to address the problems of interest to this thesis is still limited, and further work needs to be conducted towards that direction. For instance, some of them need further validation or testing, with a more in-depth analysis of their specific capabilities and predictive performance.

Therefore, in this thesis, we conduct new research to further advance the state of knowledge in the fields of rock burst prediction and of squeezing prediction, focusing on four aspects that could improve the above mentioned analytical, numerical, empirical and machine learning methods; in particular, we contribute with (i) new approaches that could predict underground tunnel hazards probabilistically, so that they can be applied for risk analyses and updated with new databases; (ii) new approaches that can deal with incomplete data to fully employ them in the analyses; (iii) the development of simple equations or indices based on machine learning methods that can be easily applicable on site; and (iv) the comparison of different machine learning techniques for an in-depth assessment of their accuracy and stability performance.

\subsection{Objectives}

The objective of the research presented in this dissertation is to use empirical observations of tunneling problems (related to rock burst and to squeezing) to develop 
simple approaches for long-term and short-term prediction of such hazards in real projects. Ease of use with preliminary data is important to our approach, so that such methods can be used in practice; and this is the reason why the methods are developed using simple input information that is commonly available at the early stage of projects. The specific objectives of the dissertation can be summarized as follows:

(1) A linear classifier for long-term probabilistic prediction of rock burst hazard: We aim to propose a new simple and easily applicable approach to predict rock burst based on the use of linear classifiers. Our aim is to predict the probability of rock burst in the long-term using simple parameters that are commonly available at the early stages of projects. Such new tool could be employed for risk analyses of rock burst at the initial stage of the projects.

(2) Prediction of rock burst with incomplete data using Bayesian Networks: Bayesian Networks are a useful tool to take advantage of the knowledge included within a given database, and they can be employed to predict underground hazards such as rock burst. In addition, we want to analyze how the BN structure affects the results, as this aspect is not yet fully understood. To that end, several BN classifiers are established and compared to probabilistically predict the occurrence of rock burst.

(3) Evaluating short-term rock burst damage using Rock Engineering Systems: We aim to present a damage index — the so called rock burst damage index, or RBDI — that could be used to estimate the short-term rock burst hazard. The RBDI is developed using the Rock Engineering Systems (RES) methodology, hence allowing us to consider the different interrelations between the parameters that affect the rock burst behavior. 
(4) Predicting tunnel squeezing with machine learning techniques: We aim to train, validate, compare and evaluate different machine learning techniques to predict tunnel squeezing. The optimal model, combined with the optimal algorithm, is to be proposed to better predict the likely occurrence of rock tunnel squeezing preliminarily.

\subsection{Outline of the thesis}

The dissertation is divided into seven chapters.

In Chapter 2, three databases employed for the analyses conducted in the dissertation are introduced.

In Chapter 3, we present a linear classifier to predict the probability of long-term rock burst occurrence. First, several models with two or three parameters are trained and validated using the extensive database of rock burst case histories compiled from the literature. Then, the optimal 2D and 3D models are analyzed and the probability lines and planes are illustrated. The final proposed model is compared with other empirical models, showing that our new model has the best predictive ability, and that it could be used in risk analyses.

In Chapter 4, we present a novel application of Bayesian networks to predict rock burst occurrence with three different classifiers. Using the database developed in Chapter 2, the ability of this technique to deal with incomplete data is emphasized, so that our collected database could be fully employed to train the $\mathrm{BN}$ using the Expectation Maximization (EM) algorithm; then, the learned Tree augmented Naïve Bayes (TAN) classifier can be employed to predict the probability of rock burst for a given set of evidences or cases.

In Chapter 5, we propose a new rock burst damage index to estimate short-term rock 
burst hazards. A large database including 13 parameters related to rock burst is employed, and several parameters are selected to establish the training set for the ANN algorithm. The Rock Engineering Systems approach is extended to develop a new rock burst hazard index (RDSI), which can be employed to estimate the likely scales of rock burst damage.

In Chapter 6, we investigate the performance of several common machine learning tools that have not yet been employed to predict rock tunnel squeezing. To that end, a database of 166 tunneling case histories compiled from the literature is employed, and several models are analyzed in terms of their predicative accuracy and stability.

Chapter 7 presents a summary of the major findings from the dissertation and some suggestions for future work. 



\section{DATABASE DESCRIPTION}

In this chapter, three databases employed in the dissertation are introduced. The databases correspond to different underground tunnel hazards that are analyzed in later chapters of the dissertation: long-term and short-term rock burst; and tunnel squeezing. Each database contains a set of good-quality case histories for the model training and validation. Several parameters are included in each case history, and in some occasions, the information about some of them is incomplete. The three databases are discussed in the following sections, and the complete databases are listed in the Appendices.

\subsection{Database for long-term rock burst prediction}

In order to propose alternative approaches to estimate the likelihood of long-term rock burst, we compiled a database of 135 case histories from different types of underground excavations from all over the world, in which the occurrence (or not) of rock burst has been recorded. Out of the 135 case histories within the database, 83 correspond to rock burst cases and 52 to non-rock burst cases. 
The database is developed based on observations reported by Zhou et al. (2012), Zhao et al. (2007), Guo et al. (2008) and some unpublished technical reports. Note that, although the literature seems to agree in that the mechanisms leading to rock burst are the same in coal and non-coal cases, the consequences could be different due to their specific features. However, due to the limited size of the available database, and because we are mainly interested in the occurrence of rock burst (and not so much on its consequences), and in agreement with previous researchers (see e.g. Feng and Wang (1994) and Gao (2015)), we choose to treat both types of rock burst together in our analyses.

Each record (i.e., case history) in the database contains five fields that correspond to the five main aspects that, based on the literature review conducted, could mainly influence rock burst. To be able to conveniently establish a classifier in later chapters, those five aspects are divided into two types — 'environmental' and 'rock intrinsic' based on their characteristics. The parameters considered, their descriptions and types are listed in Table 2.1. Note, however, that information about such parameters or fields might be incomplete, so that not all case histories contain information about all parameters. This is the reason why, in the discussion of the analyses conducted, the number of case histories employed might be different for the different models considered. (See the different models developed in Chapter 3.)

Table 2.1 Parameters in the database for long-term rock burst prediction

\begin{tabular}{|c|c|c|}
\hline Parameter & Physical description & Types \\
\hline $\mathrm{H}$ & Buried depth of the tunnel & Environmental \\
\hline MTS & $\begin{array}{l}\text { Maximum tangential stress of the } \\
\text { surrounding rock }\end{array}$ & Environmental \\
\hline UCS & Uniaxial compressive strength of the rock & Rock intrinsic \\
\hline UTS & Uniaxial tensile strength of the rock & Rock intrinsic \\
\hline $\mathrm{W}_{\text {et }}$ & Elastic energy index & Rock intrinsic \\
\hline
\end{tabular}


The Boxplot of the collected database is given in Figure 2.1 and statistics of parameters are shown in Table 2.2. Black solid points in Figure 2.1 indicate the "outliers" (or extreme cases) of each distribution, the horizontal bold lines inside the boxes represent its median values, and the bottom and top lines of each box indicate the first and third quartiles. Table 2.3 lists the correlations coefficients (and the corresponding P-values) among the parameters; it can be observed that only the UCS-UTS and UCS-Wet relationships have correlation coefficients higher than 0.6. The histograms of the five parameters are shown in Figure 2.2. The complete database of case histories employed for long term rock burst prediction is listed in Appendix A.

Table 2.2 Descriptive statistics of the input parameters for case histories within the long-term rock burst database

\begin{tabular}{lllllll}
\hline Parameter & Available & Missing & Min & Max & Mean & $\begin{array}{l}\text { Standard } \\
\text { deviation }\end{array}$ \\
\hline H [m] & 119 & 16 & 100 & 1140 & 705.97 & 274.53 \\
MTS [MPa] & 100 & 35 & 2.6 & 167.2 & 56.28 & 33.21 \\
UCS [MPa] & 134 & 1 & 2.9 & 263 & 97.32 & 54.69 \\
UTS [MPa] & 123 & 12 & 0.38 & 19.2 & 5.68 & 3.58 \\
W $_{\text {et }}$ & 117 & 18 & 1.1 & 9.3 & 4.41 & 2.05 \\
\hline
\end{tabular}

Table 2.3 Pearson Correlation coefficients (p-values) between the parameters collected in the rock burst database (The p-value is expressed in probability levels so that smaller p-levels indicate a more significant relationship)

\begin{tabular}{llllll}
\hline & $\mathrm{H}$ & MTS & UCS & UTS & $\mathrm{W}_{\mathrm{et}}$ \\
\hline $\mathrm{H}$ & 1 & $0.161(0.140)$ & $-0.245(0.008)$ & $-0.193(0.046)$ & $-0.169(0.091)$ \\
MTS & $0.161(0.140)$ & 1 & $0.216(0.031)$ & $0.207(0.039)$ & $0.293(0.003)$ \\
UCS & $-0.245(0.008)$ & $0.216(0.031)$ & 1 & $\mathbf{0 . 6 0 6 ( \mathbf { 0 . 0 0 0 } )}$ & $\mathbf{0 . 6 8 9 ( \mathbf { 0 . 0 0 0 } )}$ \\
UTS & $-0.193(0.046)$ & $0.207(0.039)$ & $\mathbf{0 . 6 0 6}(\mathbf{0 . 0 0 0})$ & 1 & $0.308(0.002)$ \\
W $_{\text {et }}$ & $-0.169(0.091)$ & $0.293(0.003)$ & $\mathbf{0 . 6 8 9 ( \mathbf { 0 . 0 0 0 } )}$ & $0.308(0.002)$ & 1 \\
\hline
\end{tabular}



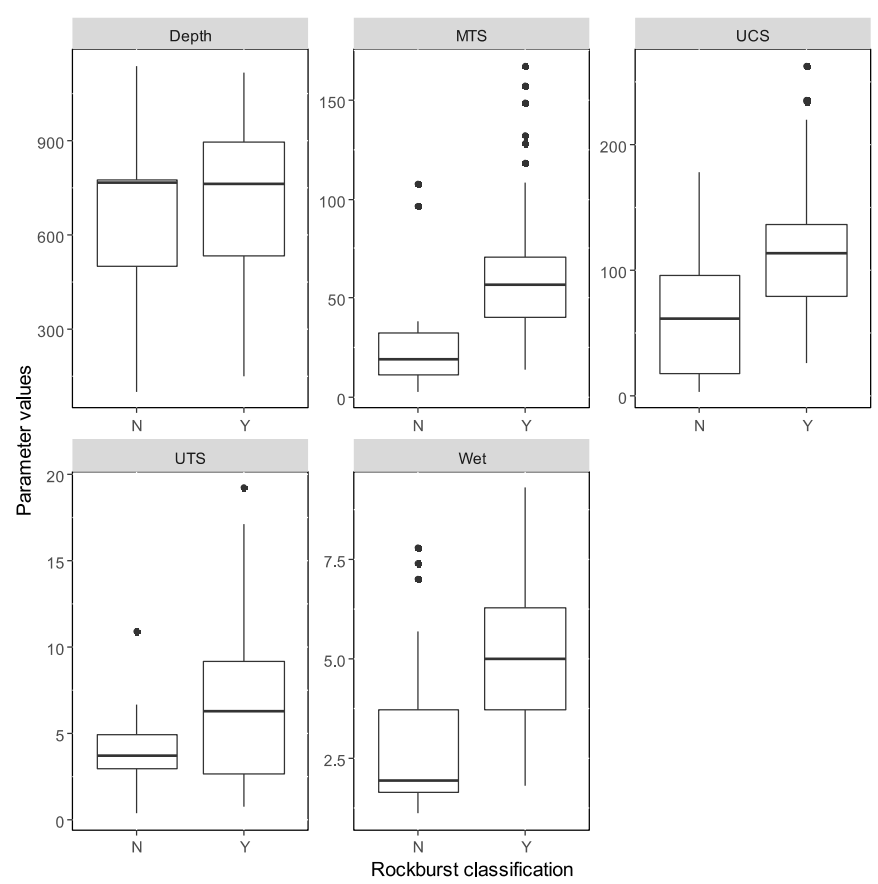

Figure 2.1 Boxplot of parameters employed for rock burst prediction (' $N$ ' represents no rock burst and ' $Y$ ' represents rock burst) 

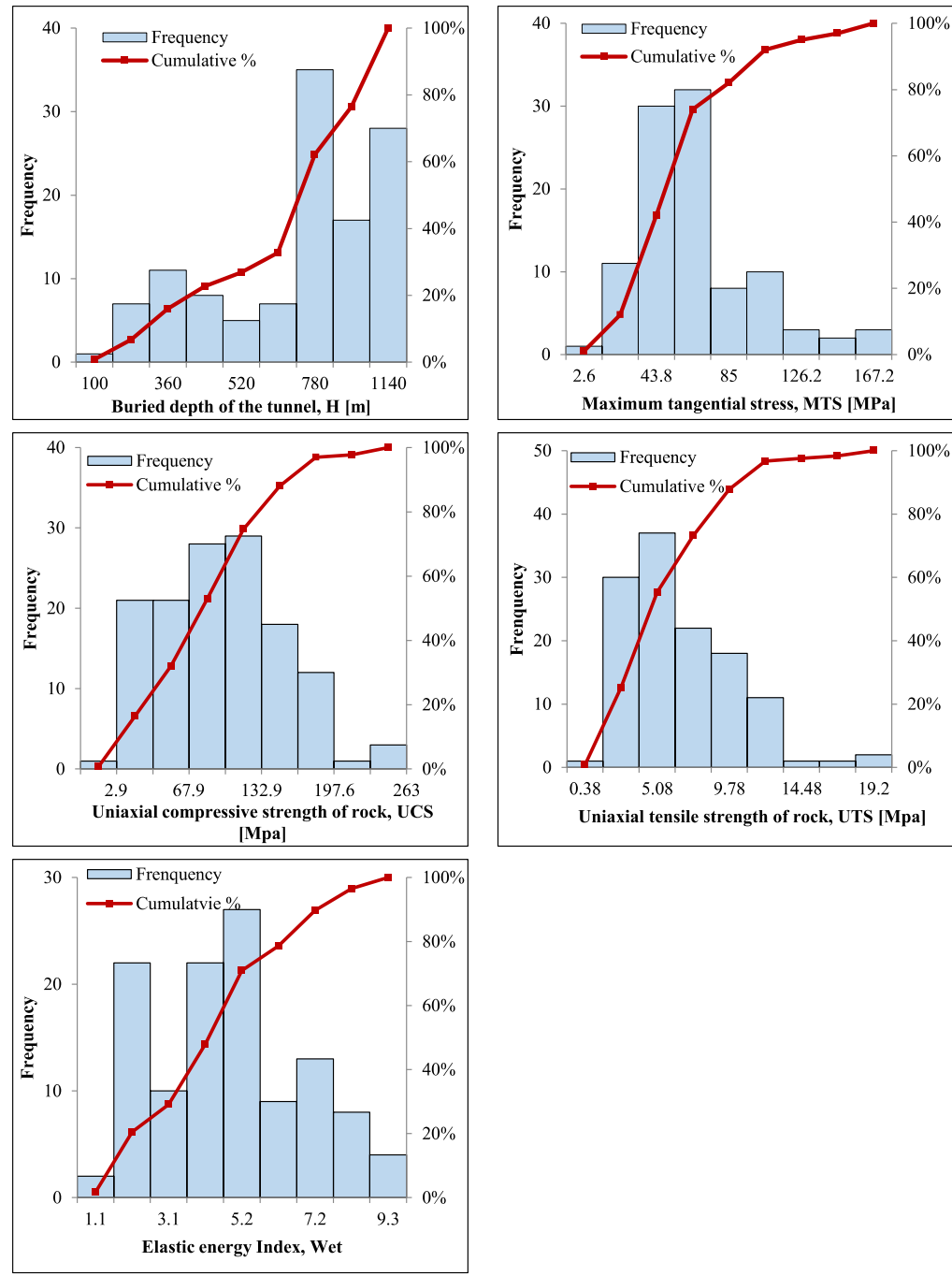

Figure 2.2 Histograms of the five parameters considered to predict the rock burst hazard

\subsection{Database for short-term rock burst prediction}

Reports of rock burst events that occurred in 13 Australian and Canadian underground rock mines have been collected by Heal (2010). 254 case histories of rock burst with different scales of intensity (R2, R3, R4 and R5) resulting from 83 recorded seismic events are collected to be later employed for the prediction work. The database contains records with fields corresponding to 15 parameters that could describe the stress condition, geological features, support, and seismic event and damage scale for each case history. The list of the 15 parameters is presented in Table 2.4. 
Table 2.4 Parameters descriptions, types and selection

\begin{tabular}{|c|c|c|c|c|}
\hline \multirow[b]{2}{*}{ Parameter } & \multirow[b]{2}{*}{ Description } & \multirow[b]{2}{*}{ Types } & \multicolumn{2}{|l|}{ Parameter selection } \\
\hline & & & $\begin{array}{l}\text { Heal (2010) and } \\
\text { Zhou et al. (2016c) }\end{array}$ & This work \\
\hline P1 & $\begin{array}{l}\text { Ratio of total maximum principal stress to } \\
\text { UCS }\end{array}$ & Cause & $\bullet$ & $\bullet$ \\
\hline $\mathrm{P} 2$ & $\begin{array}{l}\text { Energy capacity of the ground system that } \\
\text { reflects the type of the support system }\end{array}$ & Cause & $\bullet$ & $\bullet$ \\
\hline P3 & Excavation span & Cause & $\bullet$ & $\bullet$ \\
\hline P4 & $\begin{array}{l}\text { Geology factor that considers faults, folds, } \\
\text { shear exists }\end{array}$ & Cause & $\bullet$ & $\bullet$ \\
\hline P5 & Seismic event magnitude & Cause & & $\bullet$ \\
\hline P6 & $\begin{array}{l}\text { Distance from the damage location to the } \\
\text { hypocenter of the seismic event }\end{array}$ & Effect & & \\
\hline P7 & Approximate peak particle velocity (PPV) & Cause & & $\bullet$ \\
\hline P8 & The thickness of failed rock & Effect & & \\
\hline P9 & Rock mass density & Cause & & $\bullet$ \\
\hline P10 & Damage occurs to the walls or to the backs & Effect & & \\
\hline P11 & $\begin{array}{l}\text { Ejection distance for locations where } \\
\text { damage was to the back }\end{array}$ & Effect & & \\
\hline P12 & $\begin{array}{l}\text { Estimate of energy demand that cause } \\
\text { ground support damage at the rock burst } \\
\text { location }\end{array}$ & Effect & & \\
\hline $\mathrm{P} 13$ & Detailed support types & Cause & & \\
\hline P14 & $\begin{array}{l}\text { Rock burst damage scale (RDS) estimated } \\
\text { by the ejected rock quantity }\end{array}$ & Effect & & $\bullet$ \\
\hline P15 & $\begin{array}{l}\text { Support damage scale (SDS) estimated by } \\
\text { the deformation of support }\end{array}$ & Effect & & \\
\hline
\end{tabular}

Out of such parameters, which can be broadly classified as 'cause' and 'effect' factors, we selected a subset of them which are more closely related to the prediction of the consequences associated to different rock burst events. In particular, seven 'cause' parameters and one 'consequence' parameter — the rock burst damage scale proposed by Kaiser et al. (1992) — are employed to establish the index for rock burst damage prediction. Table 2.4 also lists our selected parameters and compares them with two previous works that only considered five parameters. A more detailed explanation of such factors or parameters is described in Chapter 5.

Figure 2.3 illustrates the distributions of the 7 selected input parameters (P1, P2, P3, P4, 
P5, P7 and P9) with 4 different damage scales (R2, R3, R4 and R5). (The detailed definition of these scales is presented in Chapter 5.) It also shows that (except for the support parameter) the values of each parameter tend to increase with the damage scale. The statistics of each parameter are listed in Table 2.5. The complete database of case histories employed is reported in Appendix B.

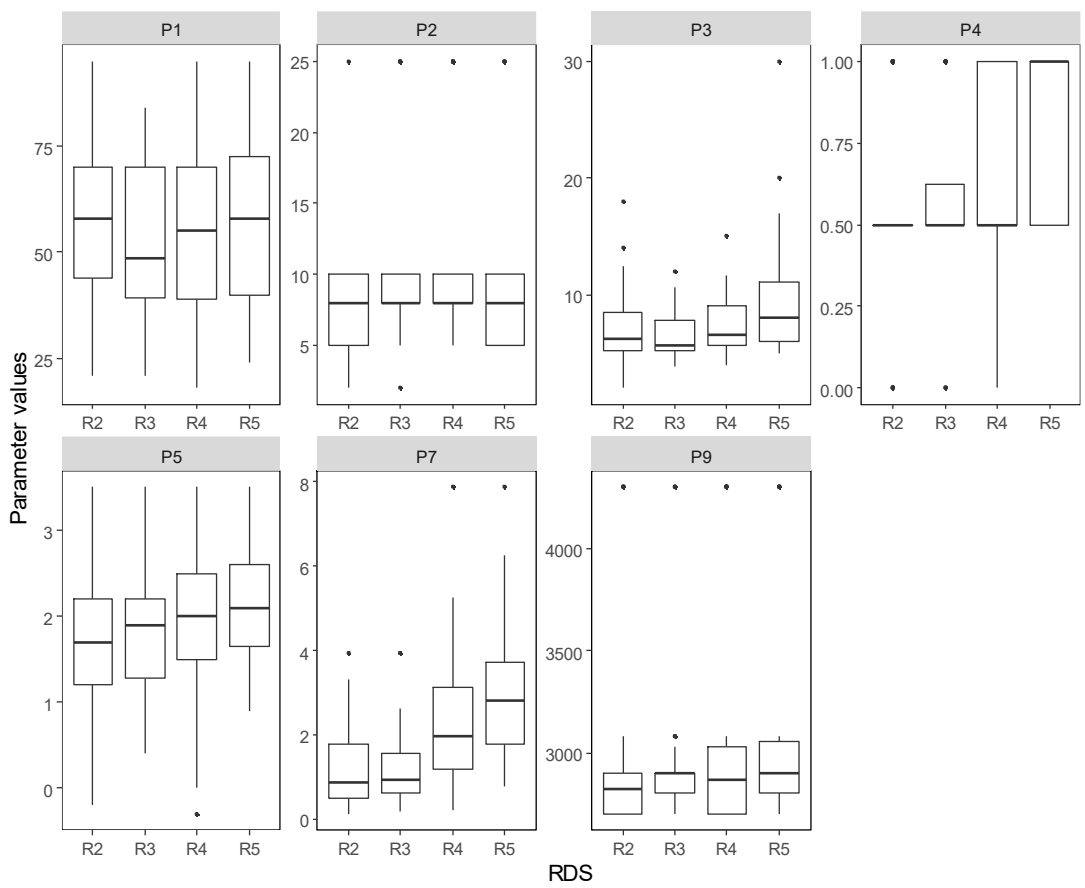

Figure 2.3 Boxplot of all the parameters for different levels of damage classification ( $R 2$ to $R 5)$ 
Table 2.5 Descriptive statistics of the input parameters for case histories within the short-term rock burst database

\begin{tabular}{lllllll}
\hline Parameter & Available & Missing & Min & Max & Mean & Standard deviation \\
\hline P1 & 254 & 0 & 18 & 95 & 54.51 & 17.81 \\
P2 & 254 & 0 & 2 & 25 & 7.88 & 4.22 \\
P3 & 254 & 0 & 2 & 30 & 7.24 & 3.01 \\
P4 & 254 & 0 & 0.5 & 1.5 & 0.90 & 0.26 \\
P5 & 254 & 0 & -0.3 & 3.5 & 1.88 & 0.81 \\
P7 & 254 & 0 & 0.12 & 7.87 & 1.66 & 1.31 \\
P9 & 254 & 0 & 2700 & 4300 & 2966.77 & 444.78 \\
\hline
\end{tabular}

\subsection{Database for tunnel squeezing prediction}

To evaluate the performance of different machine learning techniques considered in squeezing prediction, the database of case histories compiled by Jimenez and Recio (2011) and extended by Feng and Jimenez (2015) is revisited herein. It contains 166 case histories from 30 projects located in 7 countries, among which 109 are squeezing cases and 57 are non-squeezing cases. (The complete database of case histories is reported in Appendix C.)

Figure 2.4 uses a boxplot to illustrate the distributions of the five input parameters considered in the database - Tunnel span or diameter $(D)$, Rock tunneling quality index $(Q)$, Tunnel depth $(H)$, Support stiffness $(K)$ and Stress-strength ratio $(S S R)$ - and differentiating, for each parameter, the squeezing and non-squeezing cases. (Note, however, that some case histories only provide information about some parameters, hence providing incomplete information.) Figure 2.4 illustrates that all the distributions have some "outliers" (or extreme cases), although it seems apparent that, as expected, squeezing problems are more common in deep and large tunnels, specially when 
constructed with more flexible supports. The statistics of each parameter are shown in Table 2.6. Similarly, the diagonal plots in Figure 2.5 show the distribution of each individual parameter (considering together the squeezing and non-squeezing cases); whereas its off diagonal plots show their pairwise relationships, and the red line in the lower off-diagonal is the non-linear fit line of each two parameters.

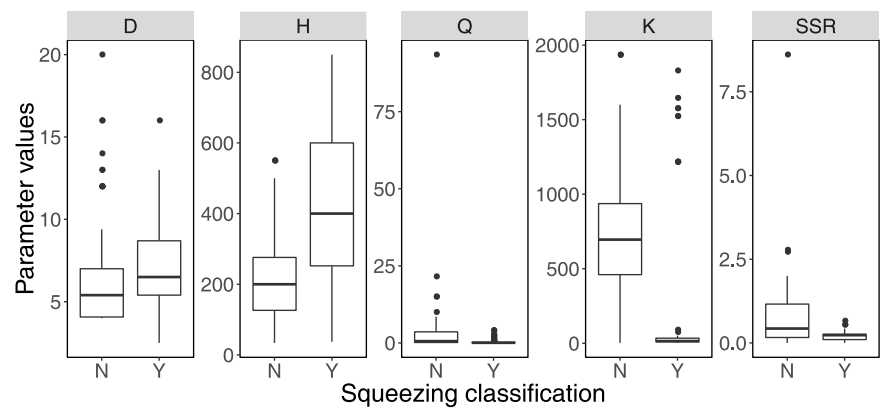

Figure 2.4 Boxplot of parameters selected for tunnel squeezing predction ("N" indicates case histories with no squeezing, and " $Y$ " indicates squeezing)

Table 2.6 Descriptive statistics of the input parameters for case histories within the squeezing database

\begin{tabular}{lllllll}
\hline Parameter & Available & Missing & Min & Max & Mean & $\begin{array}{l}\text { Standard } \\
\text { deviation }\end{array}$ \\
\hline D [m] & 165 & 1 & 2.5 & 20 & 7.22 & 3.19 \\
H [m] & 166 & 0 & 34 & 850 & 342.29 & 207.39 \\
Q & 164 & 2 & 0.001 & 93.5 & 2.21 & 10.49 \\
K [Mpa $]$ & 108 & 58 & 2.53 & 1936 & 359.05 & 573.53 \\
SSR & 95 & 71 & 0.0025 & 8.61 & 0.57 & 1.28 \\
\hline
\end{tabular}




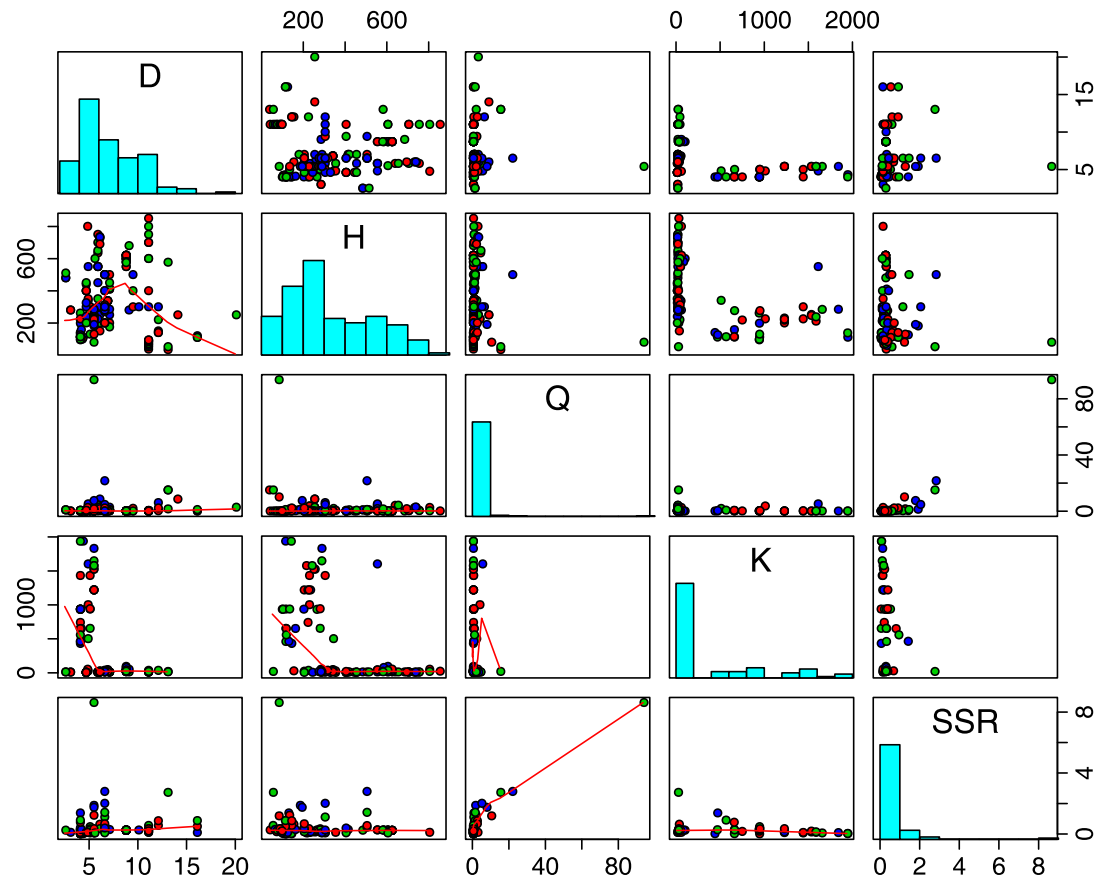

Figure 2.5 Interaction matrix of the variables in the tunnel squeezing database

\subsection{Summary}

This chapter briefly introduces three databases that will be employed for underground hazards prediction work in the following chapters. To that end, the parameters included in case histories recorded for each database are discussed; and information about the distribution of each parameter - i.e., boxplots, statistics, etc. - are demonstrated. These databases collected from the literature could represent a typical set of case histories related to each type of hazard. However, as most of them are collected at the initial stage of the projects, not all the parameters that affect the hazards are always considered, and some of them are probably even not available. So these database can be updated and improved when new case histories become available in the future, hopefully allowing researchers in this field to further improve their predictive tools. 


\section{A LINEAR CLASSIFIER}

\section{FOR LONG-TERM}

\section{PROBABILISTIC}

\section{PREDICTION OF ROCK}

\section{BURST HAZARD}

\subsection{Introduction}

Chapter 1 discusses the empirical methods that have been applied for long-term rock burst prediction. However, they are deterministic so that they cannot be easily employed in the context of risk analyses. In this chapter, we propose a novel empirical method for long-term rock burst prediction that is based on the statistical theory of logistic regression classifier. The classifier is trained and tested using a database of case histories compiled from the literature and technical reports on underground projects, and it allows us to estimate the likelihood of rock burst occurrence based on simple input data that is commonly available at early stages of project development. 


\subsection{Parameter selection for long-term rock burst prediction}

To be able to anticipate rock burst occurrence, we should incorporate (at least) most of the important aspects that influence rock burst. Some of such factors are discussed below.

\subsubsection{Buried depth of the tunnel}

Observations in real cases indicate that rock burst occurs mainly in deep rock engineering and most works consulted during the literature review agree in the observation that tunnel depth is an important factor that can affect rock burst. Therefore, and although in-situ rock stress would probably be a better predictor, the lack of information about in-situ stress in many projects, as well as the difficulties to accurately estimate in-situ rock stress at early stages of a project without expensive and time consuming in-situ tests, make us to select the buried depth of the tunnel as an alternative. (Note also that, as the excavation depth increases, the in-situ stress - which is often estimated by $\lambda H$ with $\lambda$ being the unit weight of the rock mass - also increases.) This observation motivated Hou and Wang (1989) and Pan and Li (2002) to propose empirical equations to anticipate rock burst risk based on a critical depth computed as a function of additional rock mass parameters (see Table 1.1 in Chapter 1).

\subsubsection{Maximum tangential stress of the surrounding rock}

The maximum tangential stress is often used to predict the fracture angle of rock (Aliha and Ayatollahi 2012). Similarly, Ryder (1988) concluded that the fault-slip and shear fracture mode played a dominant role on rock burst-prone conditions in Africa metal mines; whereas Qian (2014) proposed two modes of rock burst dynamic failure: one 'strain mode' resulting from the rock failure and one 'sliding mode' caused by the fault- 
slip and shear fracture events. Qian (2014) also analyzed two rock burst accidents in coal mines in China, stating that the instability due to rock burst occurrence could also be classified as 'fault-slip' and 'shear fracture' modes. Therefore, previous studies clearly illustrate that the maximum tangential stress can significantly influence the occurrence of shear fracture instabilities in tunnels, hence becoming an important parameter for rock burst prediction.

\subsubsection{Uniaxial compressive and tensile strength}

The uniaxial compressive strength and the uniaxial tensile strength are two other parameters that can influence rock burst, and they have often been applied for such task. Both are also commonly available parameters.

\subsubsection{Elastic energy index}

The Elastic energy index, $\mathrm{W}_{\mathrm{et}}$, is defined as the proportion of retained strain energy to that dissipated during a single loading-unloading cycle under uniaxial compression (Kidybiński 1981; Singh 1988). This parameter is related to the rock burst hazards, and it is also one of the most common rock tests conducted in coal mines in China (Cai et al. 2016b). Several criteria to predict rock burst based on $W_{\text {et }}$ have been proposed. Among them, the criterion proposed by Kidybiński (1981), who suggested that a value of $W_{e t} \geq 2.0$ could serve as a threshold to identify likely rock burst conditions, is probably the most commonly employed (see Table 1.1). In addition, $\mathrm{W}_{\text {et }}$ has the advantage that it can be easily measured using rock samples collected on site with a relatively simple servo-controlled laboratory test (Wang and Park 2001) whose procedures are 'normalized'(see e.g. Ulusay and Hudson (2007)); or with direct or indirect in-situ evaluations. (e.g., the "double hole" or "rebound" methods, respectively) (Kidybiński 1981). 


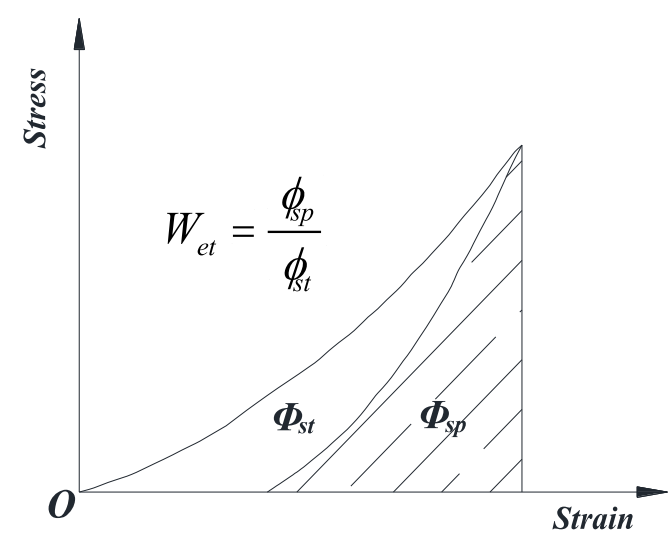

Figure 3.1 The single loading-unloading cycle of uniaxial compression and definition of $W_{\text {et }}$

\subsubsection{Burst energy release index}

The burst energy release index is a measure of the energy released at the time of rock fracture, being therefore related to the elastic energy index discussed above. In fact, the linear relation between them indicates that they can be both similarly related to rock burst (Singh 1988). However, this index was not reported in most of the case histories that we collected in Chapter 2, and we have therefore not considered it in the linear classifier.

\subsubsection{Duration of dynamic fracture}

The duration of dynamic fracture is the time elapsed since a rock specimen reaches its ultimate strength until it is completely destroyed under uniaxial compression in the laboratory (Tuler and Butcher 1968). This parameter can also affect rock burst occurrence and, although it is been employed in rock burst analyses in China, it is often not reported for other projects from the world. For this reason, it was not employed for the training of our linear classifier model.

Although there are other factors — such as in-situ rock stress (mentioned above), water conditions or rock mass integrity — that could affect rock burst, they are not considered 
herein due to the absence of a sufficient number of reliable case histories that provide information about them in the context of rock burst occurrence. When more information about them are available, they could probably be included in future models for a better prediction work.

\subsection{Linear classifier for rock burst prediction}

\subsubsection{Definition}

Let us suppose that the input vector is given by $X=\left(X_{1}, X_{2}, X_{3}, X_{4}, \ldots X_{n}\right)$; and that the output can be expressed as a linear function (Jordan, 2003):

$$
Y=\theta^{T} X+\varepsilon
$$

where $\theta$ is a parameter factor or weight, and $\varepsilon$ is a deterministic error term or bias.

For a two-class classification problem, one can visualize a linear classifier as splitting a two, three or high-dimensional input space with a hyperplane.

The two broad classes of methods mentioned in Chapter 1 -generative and discriminative methods - can be employed to determine the parameters of a linear classifier (Ng and Jordan 2002). Here, we employed a logistic regression approach, which is a type of discriminative model in which a maximum likelihood estimation (MLE) of the parameters in $\theta$ is conducted to 'learn' the model. Details of the formulation are briefly described below.

\subsubsection{Logistic function}

A logistic function or logistic curve is a common function with a "s" shape that can be seen in Figure 3.2. Mathematically, it can be expressed as (Verhulst 1838)

$$
f(x)=\frac{L}{1+e^{-k\left(x-x_{0}\right)}}
$$


where $e$ is the natural logarithm base, $x_{0}$ is the abscissa value of the sigmoid's midpoint, $L$ the curve's maximum value, and $k$ is a parameter that controls the steepness of the curve.

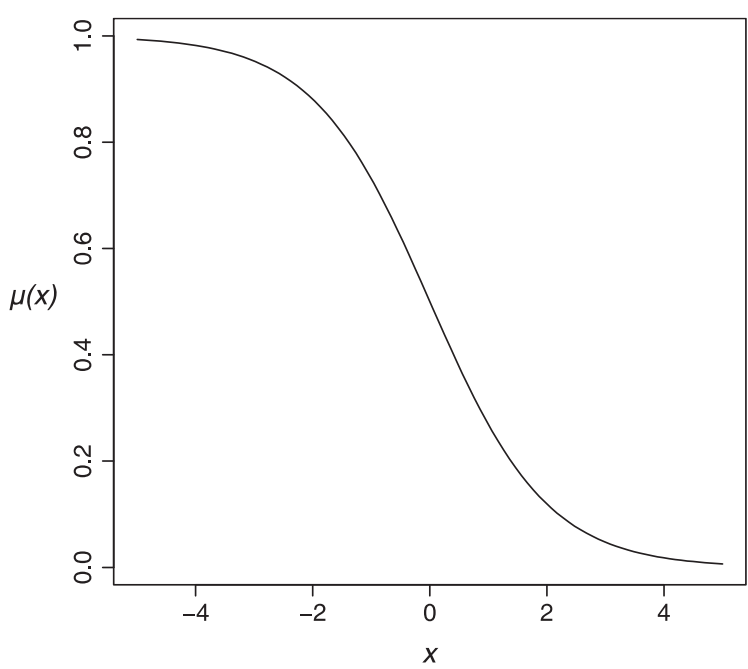

Figure 3.2 The logistic function (Sigmoid function)

The sigmoid function is a special case of the logistic function given by the following equation:

$$
f(x)=\frac{1}{1+e^{-x}}
$$

This formula will be employed to transform values into a probability scale here. In Chapter 5, the Backpropagation algorithm employed in the ANN also employs it to control the range of output values in hidden layers and in the output layer.

\subsubsection{Application in rock burst prediction}

Statistical classifiers aim to assign 'labels' to observations based on their characteristic features; in other words, they aim to identify the "class" or group to which observations belong. In the context of rock burst prediction, our statistical classifiers are trained to assign a discrete-valued random variable, $Y$, that represents the possible rock burst occurrence — "rock burst" or "no rock burst"-, based on the observation of a set of 
variables, $X$, defined in terms of the input parameters discussed above. The main difference with many of the previous efforts to address this problem is that assignment can be conducted probabilistically, using the conditional probability $P(Y \mid X)$, computed as explained below.

Our classifier is a discriminative model (Mitchell 2015), so that it aims to maximize the quality of its predictions for the training set, using the logistic function to construct a 'map' between physical features and rock burst probabilities. In particular, if we use $X$ $=\left(X_{1}, \ldots, X_{n}\right)$ to denote the input vector that includes information about (some of) the parameters that influence rock burst described above - i.e., H, W $\mathrm{et}_{\text {et }}$ MTS, UTS and/or UCS - , then the conditional probability of the class label, $Y$, can be computed based on a set of observations on $X$, as $P(Y=y \mid X=x)$. And, since $Y$ is a Bernoulli-type random variable ( $Y=1$ indicates "rock burst", whereas $Y=0$ indicates "no rock burst"), the conditional probability can be expressed as

$$
p(y \mid x)=f(x)^{y}(1-f(x))^{1-y}
$$

where $f(x)$ is the parameter of the Bernoulli distribution - note that it is a function of input data-, with $f(x)=p(y=1 \mid x)=E(y \mid x)$.

Furthermore, we assume that $f(x)$ can be computed using the logistic function and a linear transformation of the input features, as

$$
f(x)=\frac{1}{1+\exp \left(-\theta^{T} x\right)}
$$

where $\theta$ is the parameter vector of the linear classifier.

To 'learn' the parameter vector, a maximum likelihood estimate approach originally proposed by Ronald Fisher (Pfanzagl 1994) is common. It is a method to estimate the parameters of a statistical model given observations, by finding the parameter values 
that maximize the likelihood of the model producing the observations, given such parameters. In particular, for a random sample with an assumed probability distribution that depends on a set of unknown parameters, $\theta$, a good set of parameter estimates would be that maximizing the probability (or the likelihood) of getting the observed data. If we assume that the random sample is given by $X=\left(X_{1}, X_{2}, \ldots, X_{n}\right)$, and that the probability density function of each $X_{i}$ is $f\left(x_{i} ; \theta\right)$, then the joint probability mass function of $X_{1}, X_{2}, \ldots, X_{n}$ is:

$$
L(\theta)=P\left(X_{1}=x_{1}, X_{2}=x_{2}, \ldots, X_{n}=x_{n}\right)=f\left(x_{1} ; \theta\right) \cdot f\left(x_{2} ; \theta\right) \cdots f\left(x_{n} ; \theta\right)=\prod_{i=1}^{n} f\left(x_{i} ; \theta\right)
$$

In practice, Eq. (3.6) is often more convenient by expression using the natural logarithm of the likelihood function, called the log-likelihood:

$$
\ln L\left(\theta ; x_{1}, x_{2}, \ldots, x_{n}\right)=\prod_{i=1}^{n} \ln f\left(x_{i} ; \theta\right)
$$

Then, in light of the basic idea of maximum likelihood estimation, one can treat $L(\theta)$ or $\ln L(\theta)$ as a function of $\theta$, and find the value of $\theta$ that maximizes it. The least squares algorithm is proposed next to estimate $\theta$.

In our case, assuming independence between observations in the training data set, we can define the likelihood as

$$
p\left(y_{1}, \ldots, y_{N} \mid x_{1}, \ldots, x_{N}, \theta\right)=\prod_{i=1}^{N} f\left(x_{i}\right)^{y_{i}}\left(1-f\left(x_{i}\right)\right)^{1-y_{i}}
$$

and, taking logarithms to facilitate the optimization, we obtain the log-likelihood as

$$
\log p=\log \left(y_{1}, \ldots, y_{N} \mid x_{1}, \ldots, x_{N}, \theta\right)=\sum_{i=1}^{N}\left\{y_{i} \log f\left(x_{i}\right)+\left(1-y_{i}\right) \log \left(1-f\left(x_{i}\right)\right)\right\}
$$

The log-likelihood in Eq. (3.9) could be optimized using a wide variety of methods, but Newton-Raphson methods — which are iterative methods that employ first and second 
order derivatives - and, in particular, the iteratively reweighted least squares (IRLS) algorithm, are commonly employed due to their good performance (Jimenez and Recio 2011). Newton-Raphson algorithms are iterative algorithms in which the $(i+1)$ th estimation of parameters is based on the previous one, and also on the information about the first and second derivatives with respect to the (unknown) parameters. The general expression is given by:

$$
\theta^{(i+1)}=\theta^{i}-H^{-1} \nabla_{\theta} L
$$

where $\theta$ is the parameter to be estimated, $H$ is the matrix with second derivatives of $\theta$ (the Hessian matrix), $\nabla_{\theta}$ is the vector with first derivatives of $\theta$ (the gradient vector), and $L$ is the log-likelihood function. The first and second derivatives can be calculated numerically using Eq. (3.9), and Eq. (3.10) can be iteratively solved (using MATLAB or any other programming language) under a certain convergence tolerance. The final expression is:

$$
\theta^{i+1}=\theta^{i}+\left(X^{T} W_{i} X\right)^{-1} X^{T}\left(y-f\left(x_{i}\right)\right)
$$

where $X$ is the matrix of observations (with an additional column of ones to provide an independent item); $W=\operatorname{diag}\left\{f\left(x_{1}\right)\left(1-f\left(x_{1}\right)\right), \ldots, f\left(x_{N}\right)\left(1-f\left(x_{N}\right)\right)\right\}$ is a diagonal weight matrix that changes from iteration to iteration — hence the algorithm's name$y$ is a $N \times 1$ dimensional vector with the observed outcomes of "rock burst" or "no rock burst" occurrence for each input; and $f\left(x_{i}\right)$ is another $N \times 1$ vector with the Bernoulli's model parameters computed with Eq. (3.5) for each input vector with the current value of the parameter vector, $\theta^{i}$.

\subsection{Results and discussion}

\subsubsection{Model training and probability calculation}


The algorithm described in Section 3.3 has been implemented in MATLAB, and several models with different types of 'environmental' (or 'external') and 'rock intrinsic' parameters have been tested to assess their performance and predictive capabilities.

For simplicity, we only construct models with two or three input parameters out of the five parameters discussed above, so that at least one parameter is chosen from among the 'environmental' parameters (H or MTS) and at least one is chosen from the 'rock intrinsic' parameters ( $\mathrm{W}_{\text {et, }}$ UCS or UTS). The possible models using these parameters, and their training results, are listed in Table 3.1. Results show that as expected, models with three parameters perform better than models with two. Note also that the models that use MTS as 'environmental' parameter obtain lower error rates, but their prediction are biased, which makes us to suggest that other non-biased models be used instead (although they are biased towards the conservative side, with non-rock burst cases being predicted as rock burst, unbiased models are considered preferable in the context of risk analyses). And it can also be noted that models that do not consider $\mathrm{W}_{\text {et }}$ and MTS have consistently higher error rates (above $20 \%$ ).

Next, we discuss two of the models in Table 3.1 that, due to their simplicity and performance, were considered preferable to predict rock burst. The first model (referred to as Model A) considers only two input (predictive) variables: $\mathrm{W}_{\text {et }}$ and $\mathrm{H}$. Model B contains three input variables: $\mathrm{W}_{\text {et, }} \mathrm{UCS}$ and $\mathrm{H}$. 
Table 3.1 Possible models using parameters collected

\begin{tabular}{|c|c|c|c|c|}
\hline \multicolumn{2}{|c|}{ Types of Parameters } & \multirow{2}{*}{ Error cases } & \multirow{2}{*}{ Case number } & \multirow{2}{*}{ Error rate } \\
\hline Environmental & Rock intrinsic & & & \\
\hline $\mathbf{H}$ & $\mathbf{W}_{\text {et }}$ & $10+10$ & 101 & $19.8 \%$ \\
\hline $\mathrm{H}$ & UCS & $20+8$ & 118 & $23.7 \%$ \\
\hline $\mathrm{H}$ & UTS & $25+5$ & 107 & $28.0 \%$ \\
\hline MTS & $\mathrm{W}_{\text {et }}$ & $10+2$ & 100 & $12.0 \%$ \\
\hline MTS & UTS & $10+2$ & 100 & $12.0 \%$ \\
\hline MTS & UCS & $11+2$ & 100 & $13.0 \%$ \\
\hline H, MTS & $\mathrm{W}_{\text {et }}$ & $4+4$ & 85 & $9.4 \%$ \\
\hline H, MTS & UCS & $7+4$ & 85 & $12.9 \%$ \\
\hline H, MTS & UTS & $8+2$ & 85 & $11.8 \%$ \\
\hline $\mathbf{H}$ & $\mathbf{W}_{\text {et }}, \mathbf{U C S}$ & $2+4$ & 99 & $6.1 \%$ \\
\hline MTS & UCS, $W_{\text {et }}$ & $10+2$ & 100 & $12.0 \%$ \\
\hline $\mathrm{H}$ & $\mathrm{W}_{\text {et }}$, UTS & $4+4$ & 89 & $9.0 \%$ \\
\hline $\mathrm{H}$ & UCS, UTS & $23+7$ & 107 & $28.0 \%$ \\
\hline MTS & $\mathrm{W}_{\text {et }}, \mathrm{UTS}$ & $10+3$ & 100 & $13.0 \%$ \\
\hline MTS & UCS, UTS & $9+2$ & 100 & $11.0 \%$ \\
\hline
\end{tabular}

Notation: For the catalogue of error cases, the first figure indicates that observations assigned a rock burst label when they actually presented non-rock burst conditions (false-positive); whereas the second indicates observations for which a non-rock burst label was assigned even though the rock burst occurred in reality (false-negative).

Based on the above discussion about model construction, the linear classier for Model A is developed using the following input observation matrix:

$$
X=\left[1, X_{1}, X_{2}\right]=\left[1, W_{e t}, H\right]
$$

where $X$ is a $N \times 3$-dimensional matrix that includes a first column with a vector of ones to supply an independent term in the regression, and $N$ is the number of observations in the collected case histories. The rock burst observation results from a $N \times 1$ dimensional vector, $Y$, in which a value of 1 represents the occurrence of rock burst and 0 represents otherwise. Similarly, the observation matrix for Model B is a $N \times 4$ dimensional matrix constructed as: 


$$
X=\left[1, X_{1}, X_{2}, X_{3}\right]=\left[1, U C S, W_{e t}, H\right]
$$

The convergence of the IRLS algorithm is fast, and the vector of parameter $\theta$ can be trained in just a few iterations. (Results presented herein are computed with a convergence tolerance of 1E-9.) As an example of the results obtained, Table 3.2 lists the iterative estimates obtained with the IRLS algorithm during training of the parameter vector for Model B. (For Model A, the algorithm converges in just six steps of computation with a final result of $\theta_{1}=-3.5092, \theta_{2}=0.9920$, and $\theta_{3}=0.0013$.)

Table 3.2 Iterative parameter estimates for the linear classifier Model B

\begin{tabular}{lllll}
\hline Iteration & $\theta_{1}$ & $\theta_{2}$ & $\theta_{3}$ & $\theta_{4}$ \\
\hline 0 & 0 & 0 & 0 & 0 \\
1 & -2.8830 & 0.0166 & 0.2271 & 0.0019 \\
2 & -4.8288 & 0.0332 & 0.3437 & 0.0029 \\
3 & -6.7756 & 0.0492 & 0.4899 & 0.0038 \\
4 & -8.2241 & 0.0600 & 0.6191 & 0.0046 \\
5 & -8.7536 & 0.0638 & 0.6747 & 0.0048 \\
6 & -8.8060 & 0.0641 & 0.6811 & 0.0048 \\
7 & -8.8064 & 0.0641 & 0.6812 & 0.0048 \\
8 & -8.8064 & 0.0641 & 0.6812 & 0.0048 \\
\hline
\end{tabular}

Once the classifier has been trained, Eq. (3.5) can been employed to develop the linear classifier for different values of probability (as indicated by $f(x)$ ), as:

$$
\theta^{T} x=\ln \left(\frac{f(x)}{1-f(x)}\right)
$$

And, particularly for Model A, we obtain:

$$
\theta_{1} \cdot 1+\theta_{2} W_{e t}+\theta_{3} H=\ln \left(\frac{f(x)}{1-f(x)}\right)
$$

where the $\theta_{i}$ values are computed with the IRLS algorithm.

As $f(x)$ is a probability, we can obtain lines in the $\mathrm{W}_{\mathrm{et}}-\mathrm{H}$ space with constant rock burst 
occurrence probability. For example, to develop a line with $50 \%$ of rock burst probability (with $f=0.5$ ), we obtain:

$$
H[\mathrm{~m}]=2699.38-763.08 W_{e t}
$$

Similarly, Model B can be transformed to:

$$
\theta_{1} \cdot 1+\theta_{2} U C S+\theta_{3} W_{e t}+\theta_{4} H=\ln \left(\frac{f(x)}{1-f(x)}\right)
$$

where the $\theta_{i}$ values correspond to the converged results listed in Table 3.2.

As a particular example, we can obtain the class-separation surface with a $50 \%$ of rock burst occurrence under the $\mathrm{W}_{\mathrm{et}}-\mathrm{UCS}-\mathrm{H}$ three dimensional space, as

$$
H[\mathrm{~m}]=1834.67-13.35 U C S-141.92 W_{e t}
$$

Figure 3.3 shows the resulting probability lines (for $f(x)=(1 \%, 10 \%, 25 \%, 50 \%, 75 \%$, 90\%, 99\%)) for Model A. And Figure 3.4 shows the class separation plane (corresponding to a $50 \%$ probability of rock burst) of Model B, which divides the input space into two regions: one in which "rock burst" is more likely than "non-rock burst" (the upper half-space); and another in which it is otherwise. Figure 3.5 is another view of the results of Model B shown in Figure 3.4, in which additional probability planes have been added (for rock burst probabilities of $1 \%, 10 \%, 25 \%, 75 \%, 90 \%$, and $99 \%$ ), and in which the angle of view have been selected so that such planes appear as lines in the plot. 


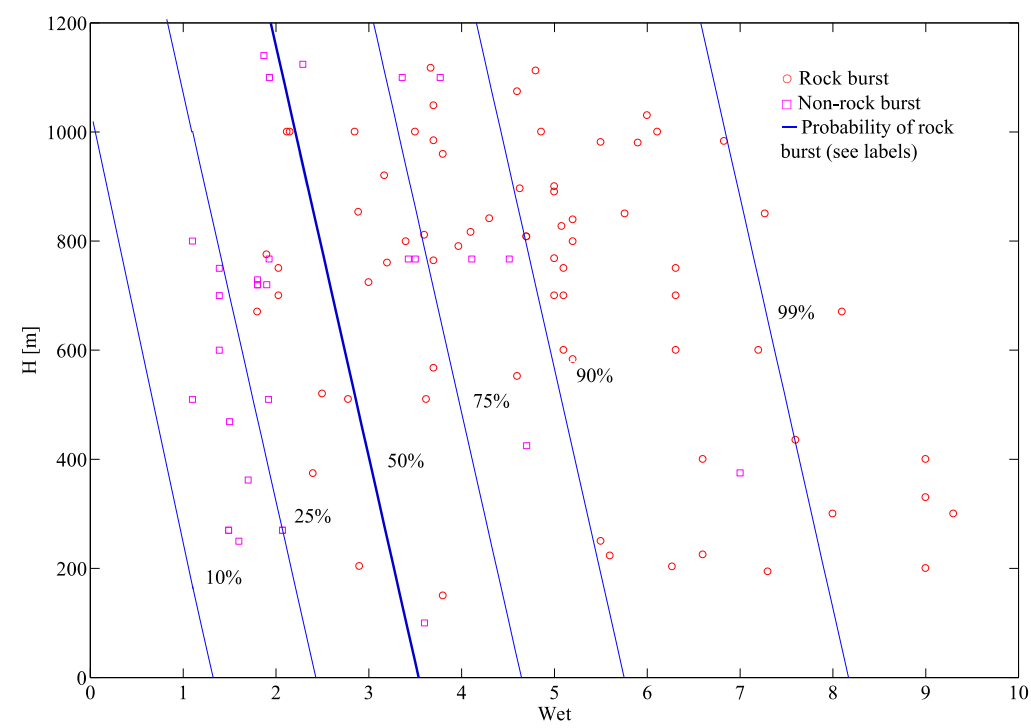

Figure 3.3 Equi-probability lines for rock burst prediction with Model A

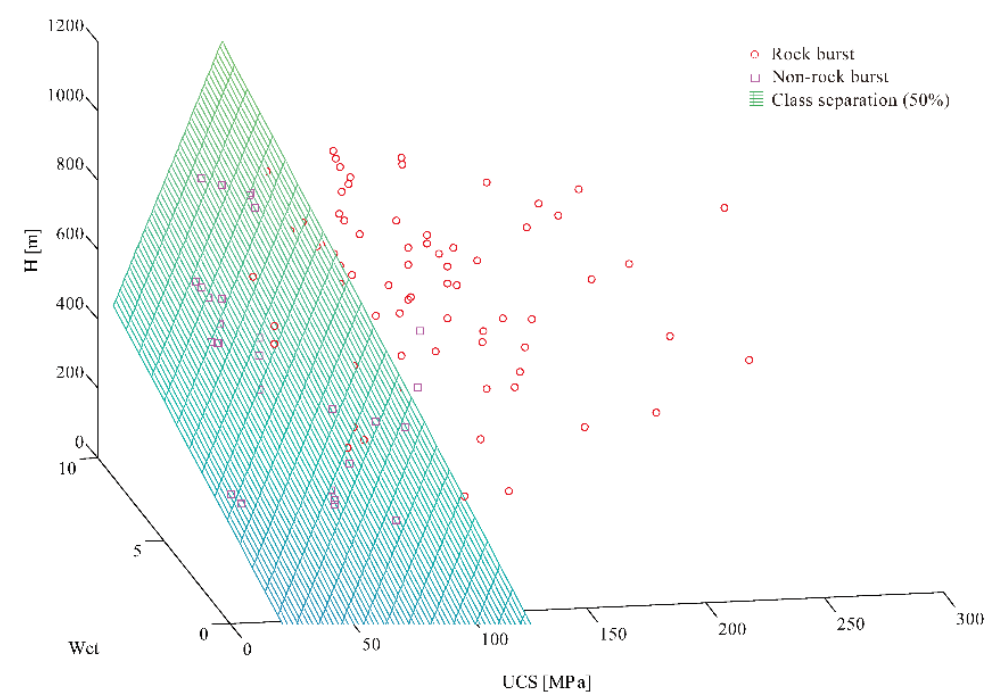

Figure $3.450 \%$ of probability plane for rock burst prediction with Model B 


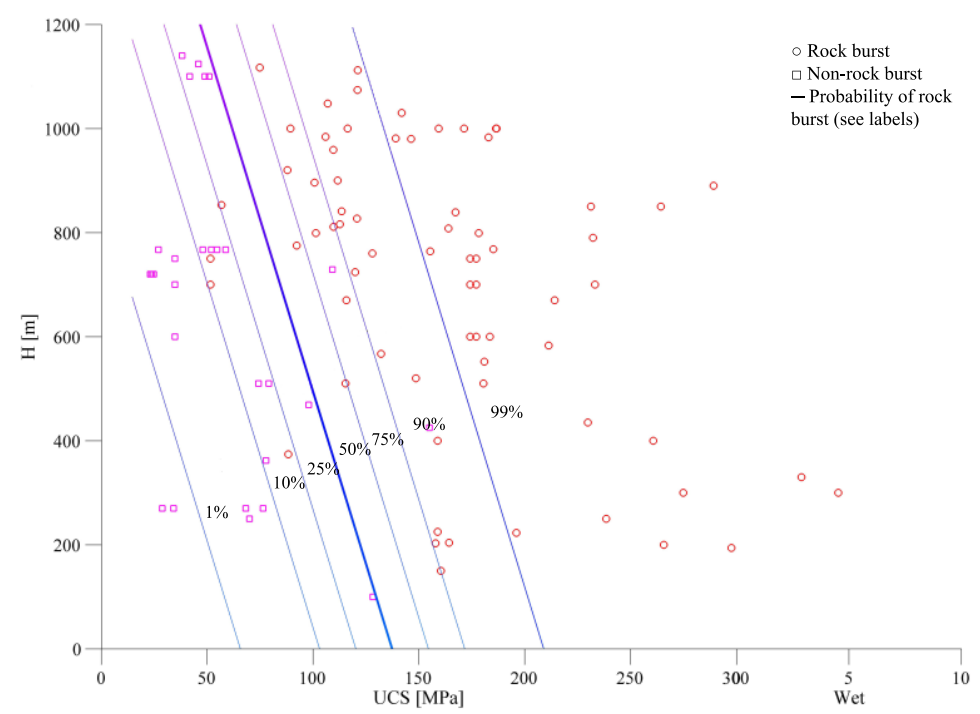

Figure 3.5 Equi-probability planes (viewed under a certain angle) for rock burst prediction with Model B

\subsubsection{Goodness of fit and predictive performance}

Goodness of fit tests aim to understand whether the model predictions accurately reflect the observed data outcomes, so that the performance of a classifier can be evaluated comparing its predictions to available observations (Dreiseitl and Ohno-Machado 2002).

\subsubsection{Deviance and Akaike's information criterion}

Deviance is a goodness of fit test for logistic regression models, based on the likelihoodratio between our fitted model and the saturated one (one in which each observation gets its own parameter). The null deviance shows how well the output is predicted by a model with an intercept only, and the residual deviance shows how well the output is predicted by the model when the predictors are included.

Akaike's information criterion (AIC; Akaike 1974) can also be used for model selection. It represents an objective methodology to select the "best" model, given some observations, among a set of candidate models; ranking the models considering both 
their fit and complexity. (Simply comparing goodness of fit is a poor model selection technique, as it is always possible to improve it increasing the number of model parameters; but too complex models should be penalized to avoid overfitting.)

Table 3.3 shows the results computed for both models. The null deviances of Models A and B are slightly different because their total number of cases histories are different, and both models decrease their deviances when predictors are added. It is also clear that Model B has a lower residual deviance and AIC than Model A, illustrating the effects of including UCS as a predictive parameter.

Table 3.3 Summary of deviances and AIC for Models A and B

\begin{tabular}{llll}
\hline Model & $\begin{array}{l}\text { Null } \\
\text { deviance }\end{array}$ & $\begin{array}{l}\text { Residual } \\
\text { deviance }\end{array}$ & AIC \\
\hline A & 122.882 & 83.141 & 81.474 \\
B & 117.930 & 41.095 & 49.095 \\
\hline
\end{tabular}

\subsubsection{Hosmer-Lemeshow (H-L) test}

The Hosmer-Lemeshow (H-L) test is another statistical test for goodness of fit for logistic regression models. It is often employed in risk prediction models. It evaluates whether the observed proportions of events within subgroups of the population match the expected proportions (Hosmer Jr et al. 2013). (In our proposed model, we divide the case histories into 10 groups for evaluation.) The test results are shown in Tables 3.4 and 3.5. The p-values for the H-L test are 0.04 for Model A and 0.41 for Model B. This suggests that Model A has a worse performance (as its p-value is the less than 0.05), whereas Model B provides better results for more reliable future predictions. 
Table 3.4 The H-L test for Model A

\begin{tabular}{|c|c|c|c|c|c|}
\hline Group & Probability zone & $\begin{array}{l}\text { Observed } \\
\text { No rock } \\
\text { burst } \\
\text { cases }\end{array}$ & $\begin{array}{l}\text { Observed } \\
\text { Rock } \\
\text { burst } \\
\text { cases }\end{array}$ & $\begin{array}{l}\text { Expected } \\
\text { no rock } \\
\text { burst } \\
\text { cases }\end{array}$ & $\begin{array}{l}\text { Expected } \\
\text { rock } \\
\text { burst } \\
\text { cases }\end{array}$ \\
\hline 1 & {$[0.149,0.25]$} & 12 & 0 & 9.58 & 2.42 \\
\hline 2 & $(0.25,0.357]$ & 6 & 3 & 6.07 & 2.93 \\
\hline 3 & $(0.357,0.548]$ & 3 & 7 & 5.52 & 4.48 \\
\hline 4 & $(0.548,0.713]$ & 2 & 8 & 3.49 & 6.51 \\
\hline 5 & $(0.713,0.824]$ & 2 & 8 & 2.21 & 7.79 \\
\hline 6 & $(0.824,0.902]$ & 4 & 6 & 1.41 & 8.59 \\
\hline 7 & $(0.902,0.927]$ & 0 & 10 & 0.83 & 9.16 \\
\hline 8 & $(0.927,0.965]$ & 0 & 10 & 0.58 & 9.42 \\
\hline 9 & $(0.965,0.982]$ & 1 & 9 & 0.24 & 9.76 \\
\hline 10 & $(0.982,0.998]$ & 0 & 10 & 0.06 & 9.94 \\
\hline
\end{tabular}

Table 3.5 The H-L test for Model B

\begin{tabular}{|c|c|c|c|c|c|}
\hline Group & Probability zone & $\begin{array}{l}\text { Observed } \\
\text { No rock } \\
\text { burst } \\
\text { cases }\end{array}$ & $\begin{array}{l}\text { Observed } \\
\text { Rock } \\
\text { burst } \\
\text { cases }\end{array}$ & $\begin{array}{l}\text { Expected } \\
\text { no rock } \\
\text { burst } \\
\text { cases }\end{array}$ & $\begin{array}{l}\text { Expected } \\
\text { rock } \\
\text { burst } \\
\text { cases }\end{array}$ \\
\hline 1 & {$[0.003492,0.04859]$} & 10 & 0 & 9.74 & 0.26 \\
\hline 2 & $(0.04859,0.2093]$ & 7 & 3 & 8.71 & 1.29 \\
\hline 3 & $(0.2093,0.5667]$ & 9 & 1 & 6.52 & 3.48 \\
\hline 4 & $(0.5667,0.8969]$ & 1 & 9 & 1.80 & 8.20 \\
\hline 5 & $(0.8969,0.94]$ & 0 & 10 & 0.78 & 9.22 \\
\hline 6 & $(0.94,0.9867]$ & 1 & 8 & 0.35 & 8.65 \\
\hline 7 & $(0.9867,0.9961]$ & 0 & 10 & 0.06 & 9.94 \\
\hline 8 & $(0.9961,0.9986]$ & 0 & 10 & 0.03 & 9.97 \\
\hline 9 & $(0.9986,0.9999]$ & 0 & 10 & 0.005 & 9.996 \\
\hline 10 & $(0.9999,1]$ & 0 & 10 & 0.0003 & 9.9997 \\
\hline
\end{tabular}

\subsubsection{Confusion matrices}

Rock burst prediction is a two class prediction problem, so that there are four possible situations which can be conveniently recorded as confusion matrices (Fawcett 2006). If the actual outcome is positive (i.e., "rock burst"), and it is classified as positive, it is counted as a true positive (TP); if it is classified as negative (i.e., "non-rock burst"), it is counted as a false negative (FN). If the actual outcome is negative and the prediction is also negative, it can be defined as true negative (TN); if it is classified as positive, it 
is counted as a false positive (FP).

Based on these definitions, and using the class-separation lines (or planes) computed with our trained classifiers in Models A and B, we obtain the performance results illustrated by the confusion matrices listed in Tables 3.6 and 3.7. The error rates for Models A and B are 19.8\% and 6.1\%, respectively. As expected (because it has only two parameters), Model A has a higher error rate, which decreases when additional 'rock intrinsic' parameters are considered (see Table 3.1), showing that considering UCS can significantly improve the predictions. Therefore, Model B is proposed as an improved logistic regression classifier that can provide better estimations, while still using simple input information.

Table 3.6 Confusion matrix of "Rock burst" prediction with Model A

\begin{tabular}{llll}
\hline Predicted & & \\
\cline { 1 - 2 } Yes & No & & \\
\hline $61(\mathrm{TP})$ & $10(\mathrm{FN})$ & Yes & Actual \\
$10(\mathrm{FP})$ & $20(\mathrm{TN})$ & No & \\
\hline
\end{tabular}

Notation: "Actual" means the real rock burst condition recorded in the database, and "predicted" means the predicted results by our proposed model. "Yes" represents rock burst occurrence, and "No" represents non-rock burst.

Table 3.7 Confusion matrix of "Rock burst" prediction with Model B

\begin{tabular}{llll}
\hline Predicted & & & \\
\cline { 1 - 2 } Yes & No & & \\
\hline $67(\mathrm{TP})$ & $4(\mathrm{FN})$ & Yes & Actual \\
$2(\mathrm{FP})$ & $26(\mathrm{TN})$ & No & \\
\hline
\end{tabular}

Notation: "Actual" means the real rock burst condition recorded in the database, and "predicted" means the predicted results by our proposed model. "Yes" represents rock burst occurrence, and "No" represents non-rock burst.

\subsubsection{Relative operating characteristic curve}

The relative operating characteristic curve (ROC; Metz 1978) is a simple empirical description that indicates all possible combinations of the relative frequencies of correct 
and incorrect decisions. A model with no discrimination ability will generate a ROC curve that follows the $45^{\circ}$ line; whereas a perfect discrimination occurs when the ROC curve follows the left and top axes of the unit square, with the true positive rate being equal to one and the false positive rate equal to zero (Pearce and Ferrier 2000).

The ROC curves of our proposed models are presented in Figure 3.6. The areas under the curve (AUC) are 0.873 and 0.961 for Models A and B, respectively. (Hosmer Jr et al. (2013) proposed that the AUC values above 0.9 represent an outstanding discrimination, whereas values between 0.8 and 0.9 represent an excellent discrimination.) Therefore our proposed Model B is also better when its performance is assessed using ROC curves and their corresponding AUC values.

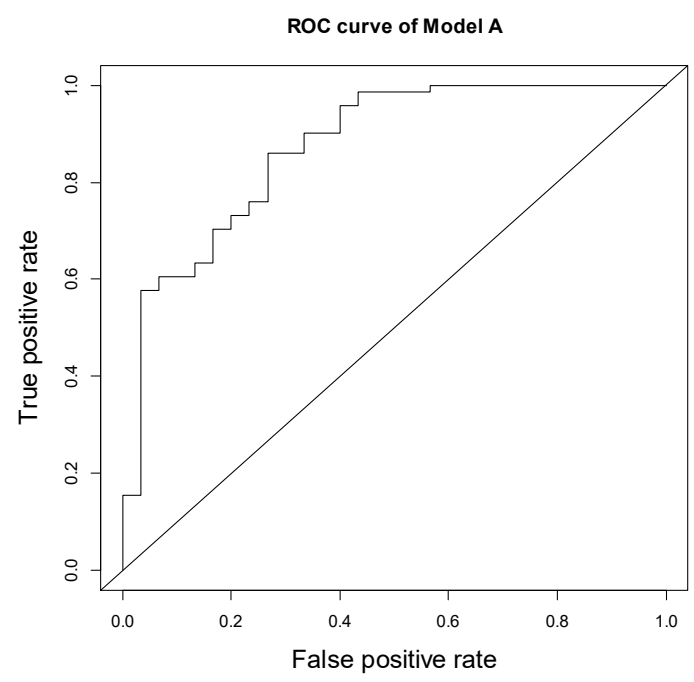

(a) Model A

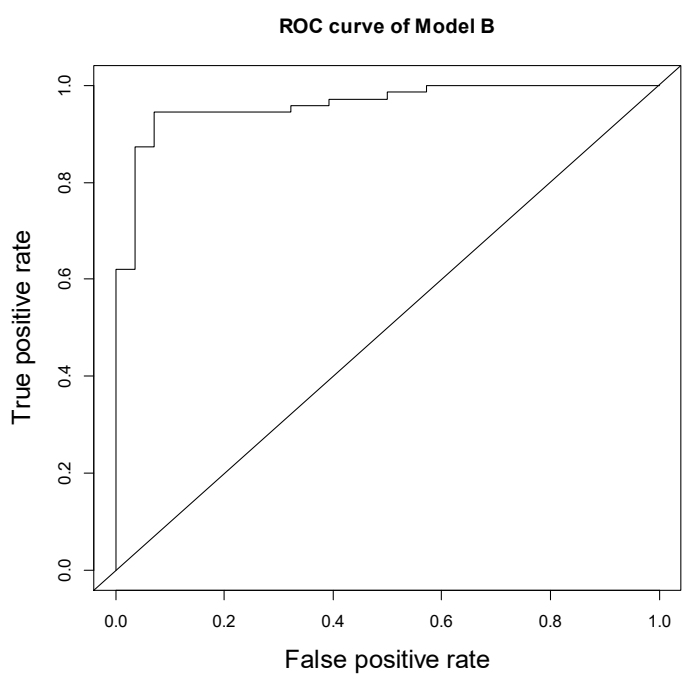

(b) Model B

Figure 3.6 The ROC curves of Models A and B

\subsubsection{Cross validation}

At the same time, a k-fold cross-validation is employed to validate Model B and to further test the performance of the linear classifier with three parameters: $\mathrm{H}, \mathrm{W}_{\mathrm{et}}$ and UCS. There are 99 case histories in the initial database, so that information about those 
three parameters and can be randomly divided into 9 groups. Then, for each group, the model is trained using the other 8 groups, and the originally selected group is used to predict rock burst occurrence with the trained linear classifier model, and to compare its prediction with the observations within the group. If this process is repeated with all groups of data, a 9-fold cross-validation exercise is obtained. Results are reported in Table 3.8, where we can note that Model B still maintains a low average error rate of $9.1 \%$.

\subsubsection{Use for risk analyses}

To illustrate the applicability of the developed models to estimate the probability of rock burst occurrence, given a set of input parameters corresponding to a new rock underground project, we can use two additional case histories: one in which the set of representative input parameters are $\mathrm{H}=700 \mathrm{~m}, \mathrm{~W}_{\mathrm{et}}=2.87$, and $\mathrm{UCS}=70.68 \mathrm{MPa}$; and another in which $\mathrm{H}=630 \mathrm{~m}, \mathrm{~W}_{\text {et }}=0.88$, and $\mathrm{UCS}=59 \mathrm{MPa}$. Then, given the set of fitted model parameters listed in Table $3.2\left(\theta_{1}=-8.8064, \theta_{2}=0.0641, \theta_{3}=0.6812\right.$, and $\theta_{4}=$ 0.0048), we can compute the probability of rock burst occurrence, $f(x)$, using Eq. (3.5), as $73.9 \%$ and $19.8 \%$ respectively. Such probability values can then be incorporated, with the corresponding estimates of costs associated to such failures (whose discussion is outside the scope of this work), into risk analyses. 
Table 3.8 9-fold cross-validation results of Model B

\begin{tabular}{|c|c|c|c|c|c|}
\hline Group & Error rates & \multicolumn{4}{|c|}{ Confusion matrices } \\
\hline \multirow{4}{*}{ No.1 } & \multirow{4}{*}{$9.1 \%$} & Pred & & & \multirow{4}{*}{ Actual } \\
\hline & & Yes & No & & \\
\hline & & 6 & 0 & Yes & \\
\hline & & 1 & 4 & No & \\
\hline \multirow{4}{*}{ No.2 } & \multirow{4}{*}{$0 \%$} & Pred & & & \\
\hline & & Yes & No & & \multirow{3}{*}{ Actual } \\
\hline & & 9 & 0 & Yes & \\
\hline & & 0 & 2 & No & \\
\hline \multirow{4}{*}{ No.3 } & \multirow{4}{*}{$18.2 \%$} & Pred & & & \\
\hline & & Yes & No & & \multirow{3}{*}{ Actual } \\
\hline & & 7 & 1 & Yes & \\
\hline & & 1 & 2 & No & \\
\hline \multirow{4}{*}{ No.4 } & \multirow{4}{*}{$0 \%$} & Pred & & & \\
\hline & & Yes & No & & \multirow{3}{*}{ Actual } \\
\hline & & 9 & 0 & Yes & \\
\hline & & 0 & 2 & No & \\
\hline \multirow{4}{*}{ No.5 } & \multirow{4}{*}{$18.2 \%$} & Pred & & & \multirow{4}{*}{ Actual } \\
\hline & & Yes & No & & \\
\hline & & 7 & 1 & Yes & \\
\hline & & 1 & 2 & No & \\
\hline \multirow{4}{*}{ No.6 } & \multirow{4}{*}{$18.2 \%$} & Pred & & & \\
\hline & & Yes & No & & \multirow{3}{*}{ Actual } \\
\hline & & 6 & 1 & Yes & \\
\hline & & 1 & 3 & No & \\
\hline \multirow{4}{*}{ No.7 } & \multirow{4}{*}{$9.1 \%$} & Pred & & & \multirow{4}{*}{ Actual } \\
\hline & & Yes & No & & \\
\hline & & 4 & 0 & Yes & \\
\hline & & 1 & 6 & No & \\
\hline \multirow{4}{*}{ No.8 } & \multirow{4}{*}{$9.1 \%$} & Pred & & & \multirow{4}{*}{ Actual } \\
\hline & & Yes & No & & \\
\hline & & 9 & 1 & Yes & \\
\hline & & 0 & 1 & No & \\
\hline \multirow{4}{*}{ No.9 } & \multirow{4}{*}{$0 \%$} & Pred & & & \\
\hline & & Yes & No & & \\
\hline & & 10 & 0 & Yes & Actual \\
\hline & & 0 & 1 & No & \\
\hline Average & $9.1 \%$ & & & & \\
\hline
\end{tabular}

\subsubsection{Discussion}

Based on the results presented in Figure 3.3, Figure 3.4 and Figure 3.5, we can observe that, as expected and in agreement with previous research (see e.g. Dou et al. 2006), the 
new classifier for rock burst prediction shows an increase of rock burst probability as $\mathrm{H}$, UCS or $\mathrm{W}_{\text {et }}$ increase. Results also illustrate that, in this case, significantly better results are obtained when an additional input parameter is included within the model: in Model $\mathrm{A}$, in which $\mathrm{W}_{\text {et }}$ is the only 'rock intrinsic' parameter considered, the result is somewhat disappointing with a relatively high error rate; whereas the predictive performance is significantly improved —using both the original set of case histories and the 9-fold cross-validation exercise - in Model B, or when UCS is added as an additional input parameter into the model.

The prediction validity of the model proposed can be assessed with the observations. For instance, we observe that the $50 \%$ rock burst probability line produces a total of $20(10+10)$ miss-classifications in the $\mathrm{W}_{\mathrm{et}}-\mathrm{H}$ space of Model A, as ten false-positives and ten false-negatives are produced; similarly, there are $6(2+4)$ miss-classifications in the $\mathrm{W}_{\mathrm{et}}-\mathrm{UCS}-\mathrm{H}$ space of Model B (see Tables 3.6 and 3.7). It can be noted that such miss-classifications are almost balanced with respect to the $50 \%$ probability line or surface, hence producing (almost) unbiased classifiers which are beneficial in risk analyses. And the other rock burst probability lines (or surfaces) also suggest that the rock burst miss-classifications tend to stay within the range expected given the obtained results.

For comparison, Table 3.9 presents the prediction results of the proposed linear classifier, together with the rock burst outcomes expected with some other common empirical methods for rock burst prediction that were presented in Table 1.1. It can be noted that our model has the lowest error rate among those considered, while still maintaining a simple equation only needs simple information that can be obtained in the initial stages of a project. 
Table 3.9 Comparison of error rates with four different empirical methods and with the linear classifier of Model B with all data and with cross-validation

\begin{tabular}{llll}
\hline Methods & Unavailable cases & Available cases & Error rate \\
\hline $\begin{array}{l}\text { Stress coefficient } \\
\text { (Russenes 1974) }\end{array}$ & 15 & 84 & $10.7 \%$ \\
$\begin{array}{l}\text { Stress coefficient } \\
\text { (Wang et al. 1998) }\end{array}$ & 15 & 84 & $17.9 \%$ \\
$\begin{array}{l}\text { Rock brittleness coefficient } \\
\text { (Wang et al. 1998) }\end{array}$ & 11 & 88 & $22.7 \%$ \\
$\begin{array}{l}\text { Elastic energy index } \\
\text { (Kidybiński 1981; Wang et al. 1998) }\end{array}$ & 0 & 99 & $13.1 \%$ \\
$\begin{array}{l}\text { Linear classifier model with all data } \\
\text { Linear classifier model with cross-validation }\end{array}$ & 0 & 99 & $6.1 \%$ \\
\hline
\end{tabular}

To assess predictor and model significance, the usual approach of hypotheses testing is employed. The Wald test statistics are computed using the R software (R Core Team 2014), obtaining that the p-values of $\mathrm{H}, \mathrm{UCS}$ and $\mathrm{W}_{\text {et }}$ are $0.00567,0.0000702$, and 0.04441 respectively. The multivariable Wald test statistics is employed to test the whole model significance, and a final p-value of 0.00007354 is obtained. The results show that our proposed Model B has a significant relationship with the predictors selected (Hosmer Jr et al. 2013). Therefore, our proposed model is considered reliable for the prediction work.

From the discussion above, we could consider Model B as the optimal classifier for rock burst prediction, as it provides a better predictive ability than previous empirical methods due to (i) its simplicity of use (or small requirements for input parameters), (ii) to its goodness of fit, and (iii) to its low error rate and unbiased predictions.

\subsection{Summary}

We present a novel application of linear classifiers for long-term prediction of rock burst. The methodology is based on simple input data that is commonly available at the early stages of design of underground structures for civil or mining applications. The 
main difference of the proposed approach with respect to traditional alternative methods is that results are computed within a probabilistic framework, which can be naturally incorporated into risk analyses. Five parameters are considered to develop the different models tested, among which two can be considered as 'environmental' or 'non intrinsic' parameters (the depth of the excavated underground facility, $\mathrm{H}$, and the maximum tangential stress, MTS); and three can be considered as 'rock intrinsic' parameters (the elastic energy index, $\mathrm{W}_{\mathrm{et}}$; the uniaxial compressive strength, UCS; and the uniaxial tensile strength, UTS).

Since models with a limited numbers of parameters are often preferred in practice, and to facilitate the visualization of results, several combinations of two and three parameter models were trained and tested using a database with 135 case histories that was compiled from the literature and from technical reports, and they were further validated using a 9-fold cross-validation exercise. Results suggest that the classifier in the $\mathrm{W}_{\mathrm{et}}{ }^{-}$ UCS-H (Model B) space should be preferentially employed in practice, as it shows significantly better goodness of fit and predictive performance (which is probably adequate for practice). In addition, the proposed model presents some improvement with respect to previously available methods, because it produces a reduced number of miss-classification cases, and because it allows the computation of probabilistic boundaries that allow designers to estimate the probability of rock burst under certain input conditions. 


\section{PREDICTING ROCK}

\section{BURST HAZARD WITH}

INCOMPLETE DATA

\section{USING BAYESIAN}

\section{NETWORKS}

\subsection{Introduction}

Chapter 1 has shown that machine learning methods have often been applied for longterm prediction of rock burst. However, one of the main difficulties to predict rock burst with existing methods (also with the proposed linear classifier models in Chapter 3 ) is that data are difficult to obtain and often incomplete. To overcome this problem, we propose a Bayesian network (BN) (Pearl 1985) to predict the occurrence of rock burst in long term discussed in Chapter 1 . As BNs have the advantage of naturally dealing 
with the conditional dependency relationships between the observed or unobserved random variables of a statistical model, hence making them an interesting choice in inference, classification and decision making problems (Aguilera et al. 2011). Although BNs have been widely employed in geotechnical engineering (Jimenez and Sitar 2006; Medina-Cetina and Nadim 2008; Xu et al. 2011; Huang et al. 2012; Schubert et al. 2012; Song et al. 2012; Sousa and Einstein 2012; Špačková et al. 2013; Borg et al. 2014; Feng and Jimenez 2015), they have not yet been employed to predict rock burst.

\subsection{Parameters chosen for the BN}

As most criteria traditionally employed in the rock burst literature only considered no more than three input parameters and hence cannot comprehensively utilize all the information about different parameters that can be collected nowadays, the proposed BN can be a powerful approach to naturally deal with data sets comprising several variables, as well as to deal with missing data and with the conditional dependency relationships between variables. Therefore, using such previous works (see Table 1.1 in Chapter 1) as guidance, we consider five parameters that have a potential influence on rock burst: buried depth of the tunnel $(\mathrm{H})$, maximum tangential stress at the surrounding rock (MTS), uniaxial tensile strength of rock (UTS), uniaxial compressive strength of rock (UCS) and elastic energy index $\left(\mathrm{W}_{\text {et }}\right)$. The detailed descriptions of these parameters are presented in Chapter 3. And, for that reason, they are not repeated here again.

\subsection{Definition of the $\mathrm{BN}$ and parameter training}

\subsubsection{Definition of the BN}

Bayesian networks are graphical models that handle probabilistic relationships among 
a set of variables in the form of directed acyclic graphs. The nodes in the network represent random variables. If two nodes have direct causal influences (i.e., if they are dependent), they will be linked by an arrow; similarly, the lack of an arrow between two variables indicates their conditional independency (Heckerman 1997).

Thus, we assume that BNs contain the variables or a set of nodes, $X=\left(x_{1}, x_{2}, \ldots x_{\mathrm{n}}\right)$. For each node $x_{i}$, if there are arrows from $S_{\mathrm{i}}$ to $x_{\mathrm{i}}, S_{\mathrm{i}}$ can be seen as the parents of $x_{\mathrm{i}}$. Then, the joint probability can be computed as

$$
P(X)=P\left(x_{1}, x_{2}, \ldots x_{n}\right)=\prod_{i} P\left(x_{i} \mid S_{i}\right)
$$

Figure 4.1 shows a simple example of a $\mathrm{BN}$ structure with six nodes, $\mathrm{x}_{1}, \mathrm{x}_{2}, \ldots \mathrm{x}_{6}$. From the equation above, the distributions of each node are $P\left(x_{1}\right), P\left(x_{2} \mid x_{1}\right), P\left(x_{3} \mid x_{1}\right), P\left(x_{4} \mid x_{1}\right.$, $\left.x_{2}\right), P\left(x_{5} \mid x_{2}\right)$ and $P\left(x_{6} \mid x_{3}, x_{5}\right)$. And the joint probability can be calculated as

$$
P\left(x_{1}, x_{2}, x_{3}, x_{4}, x_{5}, x_{6}\right)=P\left(x_{1}\right) P\left(x_{2} \mid x_{1}\right) P\left(x_{3} \mid x_{1}\right) P\left(x_{4} \mid x_{1}, x_{2}\right) P\left(x_{5} \mid x_{2}\right) P\left(x_{6} \mid x_{3}, x_{5}\right)
$$

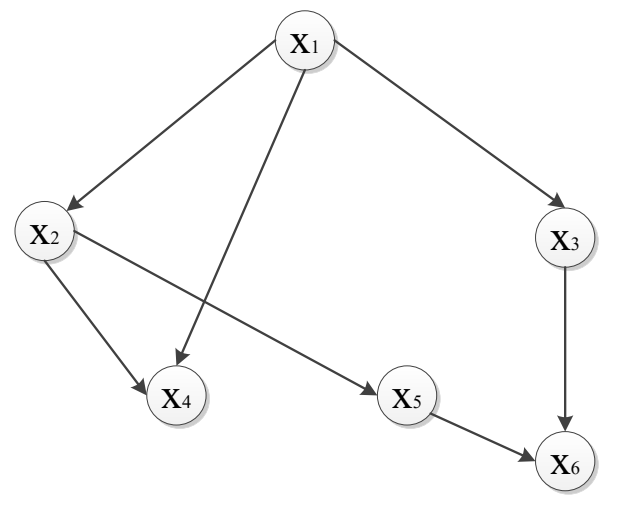

Figure 4.1 A simple Bayesian network with six parameters (based on Pearl (1985))

Bayesian networks have advantages when dealing with inference, classification and decision making problems. The main advantage is that they can handle incomplete databases and make good predictions even with small sets of case histories, which is a 
common situation at the early stages of geotechnical projects. BNs can also be readily updated when new data become available (Ticehurst et al. 2008), so that the network can be improved during project development. In addition, the learned model can be employed to compute the probability distribution of nodes given their parents and viceversa (Uusitalo 2007), so that, the 'causes' of one observation (or an accident) can also be investigated.

BN modeling can be divided into two stages: First, the structure of BN must be established based on our prior knowledge or on a structure learning algorithm. Second, the conditional probability tables (CPTs) of all the nodes in the BN should be determined, using prior knowledge or a parameter learning algorithm. Belief updating is then employed to compute the posterior probability distribution given a set of observations (Korb and Nicholson 2010).

A number of commercial software packages are available to develop BNs. The Netica software (NorsysSoftware 1998) is employed in this dissertation. One advantage of Netica is its comprehensive, flexible and user friendly graphical user interface (Uusitalo 2007).

\subsubsection{Structure learning}

There are two broad classes of algorithms to learn the structure of BNs. One class "scores" a BN based on how well it fits the data, and attempts to produce one network structure that optimizes such score. The other class uses constraints, such as independence relations, to reconstruct the structure (Margaritis 2003; Nielsen and Jensen 2009).

The Akaike's information criterion (AIC) (Akaike 1974), the Bayesian information 
criterion (BIC) (Schwarz 1978) and the minimum description length (MDL) (Grünwald 2005) are three commonly employed metrics for evaluation in score-based methods. Search algorithms are also employed to find the optimal score; the most common ones are the hill climbing algorithm (Russell and Norvig 2003), simulated annealing (Heckerman et al. 1995) and the K2 algorithm (Cooper and Herskovits 1992). For constraint-based methods, the conditional independence relationships among the attributes can be used as constraints to construct BNs. The most common algorithms for such task are the IC algorithm (Pearl and Verma 1995), the PC algorithm (Spirtes and Glymour 1991) and the GS algorithm (Margaritis 2003).

Heckerman (1997) compared both approaches, and showed that scoring-based methods have advantages. However, general scoring-based methods may theoretically result in a poor classifier (Friedman et al. 1997) and are often less efficient in practice (Cheng and Greiner 1999).

Three simple $\mathrm{BN}$ structures are employed in the dissertation, so that learning the structure is not necessary. The three different structures considered are briefly introduced here.

The naïve Bayes classifier is a simple structure in which the class label is assigned to the parent node by other nodes, having no other connections in the network. It has been used in many fields given its easy construction and efficient classification (Friedman et al. 1997; Feng and Jimenez 2015).

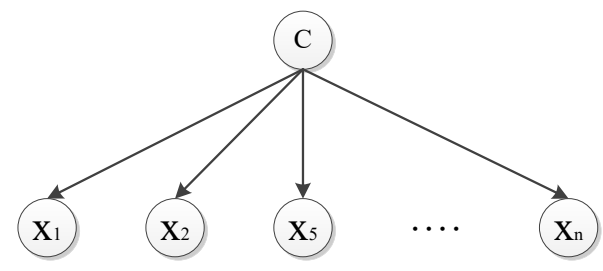

Figure 4.2 A Naïve Bayes classifier 
Figure 4.2 shows an instance of a Naïve Bayes classifier. $X_{1}, X_{2}, \ldots X_{\mathrm{n}}$ are the input variables and $C$ is the output variable with $K$ possible classes. The conditional probability can be computed using Bayes' theorem:

$$
P\left(C_{k} \mid X_{1}, X_{2}, X_{3}, \ldots, X_{n}\right)=\frac{P\left(C_{k}\right) P\left(X_{1}, X_{2}, X_{3}, \ldots, X_{n} \mid C_{k}\right)}{P\left(X_{1}, X_{2}, X_{3}, \ldots, X_{n}\right)}
$$

Assuming that input variables are independent, and that $P\left(X_{1}, X_{2}, \ldots X_{\mathrm{n}}\right)$ is constant given the input variables, the following expression can be obtained:

$$
P\left(C \mid X_{1}, X_{2}, X_{3}, \ldots, X_{n}\right) \propto P(C) \prod_{i=1}^{n} P\left(X_{i} \mid C\right)
$$

Tree augmented Naïve Bayes (TAN) classifier approximates the interactions between attributes by using a simple tree structure imposed on the Naïve Bayes structure (Friedman et al. 1997). Figure 4.3 shows an example. Learning such structures can be easily achieved by using a variation of the Chow-Liu algorithm (Chow and Liu 1968).

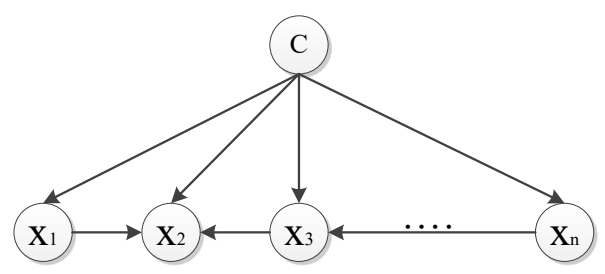

\section{Figure 4.3 A Tree augmented Naïve Bayes classifier}

BN augmented Naïve-Bayes (BAN) classifier extends the structure of TAN classifier by allowing the attributes to form an arbitrary graph, rather than just a tree. See Figure 4.4. Friedman et al. (1997) presented a minimum description length scoring method for learning a BAN. Cheng and Greiner (1999) studied a different algorithm based on a conditional independence (CI) test. 


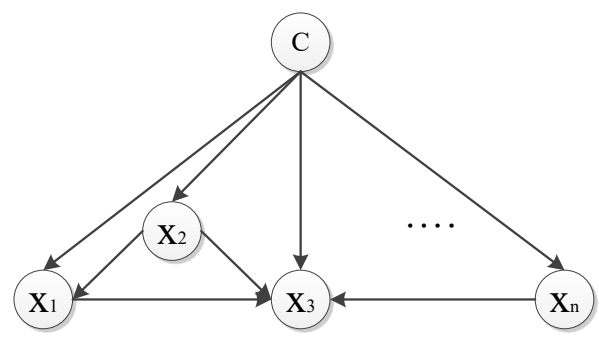

\section{Figure 4.4 A BN augmented Naïve Bayes classifier}

Provided that enough good quality data are available, BNs can be constructed to predict the probability of a complicated rock engineering hazard such as rock burst. To that end, we can divide the data set into two categories — rock burst and non-rock burstdenoted by $Y$, which $Y=0$ for non-rock burst cases or $Y=1$ otherwise; similarly, the attribute (or input) vector can be defined using the five input parameters discussed in Section 4.2, which can be expressed as $X=\left(H, M T S, U C S, U T S, W_{e t}\right)$. Then, we train the $\mathrm{BN}$ using the 135 available case histories that we have collected.

In order to define the structure of the network, the conditional independency and dependency relationships between input variables in the model are carefully considered.

The correlation coefficients between parameters within the database have been analysed to infer whether the conditional independence assumption is adequate or not. Results are listed in Table 2.3 in Chapter 2. The correlation between parameters are "weak" in most cases, except for the UTS-W et , UCS-UTS and UCS-W ${ }_{\text {et }}$ relationships, which can be defined as "moderate" to "strong" if the categorizations proposed by Dancy and Reidy (2004) and Loozen et al. (2014) are followed. (In particular, $\rho \mathrm{UTS}, \mathrm{Wet}=$ $0.308, \rho_{\mathrm{UCS}, \mathrm{UTS}}=0.606$, and $\rho_{\mathrm{UCS}, \mathrm{Wet}}=0.689$, with all other correlation coefficients being smaller, in absolute value, than 0.30.) Based on this observation, Figure 4.5 presents three alterative candidate structures for the BN that are employed herein to predict rock burst occurrence given (possible incomplete) information about the five parameters. 


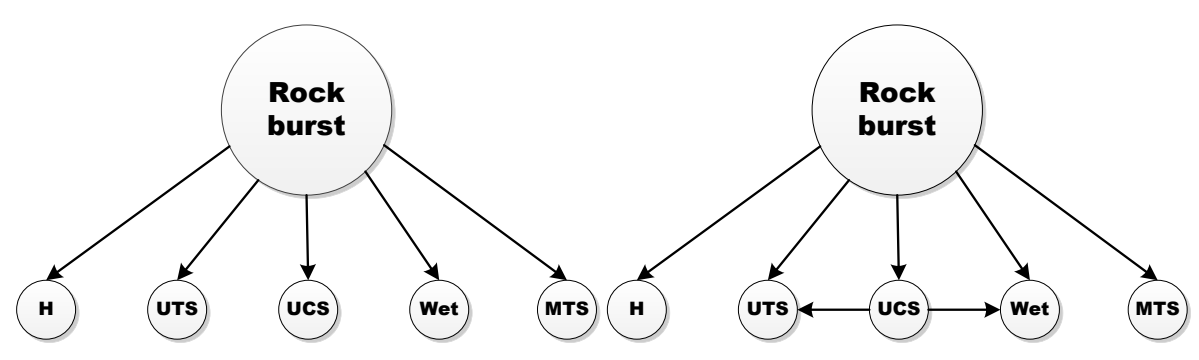

(a) Naïve Bayes

(b) Tree augmented Naïve Bayes

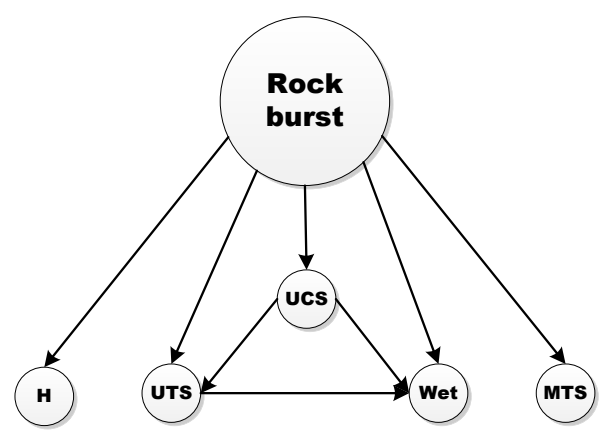

(c) BN augmented Naïve Bayes

Figure 4.5 The BN structures of three classifiers

Once the structure of the BN is defined, the intensity of the relationship —or, in other words, the conditional probability of one variable given the other - needs to be defined. For discrete variables, this is accomplished using conditional probability tables (CPTs), which quantify the conditional probability of the outcome or 'end node' having one of its possible values, given that the 'origin node' has one of its possible values. BNs have the additional feature that they can be updated as new evidence is obtained, in a process referred to as "belief updating". (The Junction Tree algorithm, discussed in Section 4.3.4, can be employed to conduct such updating.)

\subsubsection{Discretizing the continuous parameters}

The five parameters employed as input to predict rock burst are continuous. However, although theoretically possible, the practical capability of BNs to deal with continuous data is limited (Nielsen and Jensen 2009). The most common way to cope with 
continuous data is to discretize those data, and discretization is therefore employed herein.

Defining the discretization intervals is a delicate task. In this chapter, the equal frequency binning algorithm available in the software package WEKA is employed; its goal is to divide the data into intervals containing approximately the same number of cases (Hall et al. 2009). Table 4.1 summarizes the intervals defined for all input parameters considered, and it also reports the number of data in the training set that are contained within each interval.

Table 4.1 Summary of the intervals applied to the input parameters of the $\mathrm{BN}$

\begin{tabular}{ll}
\hline Parameters & Set of intervals with count \\
\hline $\mathrm{H}[\mathrm{m}]$ intervals & {$[100,200] / 4,(200,400] / 20,(400,705] / 22$,} \\
State of $\mathrm{H}$ & $(705,1140] / 73$ \\
MTS $[\mathrm{MPa}]$ intervals & Shallow, Medium, Deep, Very Deep \\
State of MTS & {$[2.6,28] / 16,(28,57.5] / 42,(57.5,167.2] / 42$} \\
UCS $[\mathrm{MPa}]$ intervals & Low, Medium, High \\
State of UCS & {$[2.9,69.15] / 44,(69.15,119] / 42,(119,263] / 48$} \\
UTS $[\mathrm{MPa}]$ intervals & Low, Medium, High \\
State of UTS & {$[0.38,3.29] / 41,(3.29,6] / 36,(6,19.2] / 46$} \\
$W_{\text {et }}[\mathrm{MPa}]$ intervals & Low, Medium, High \\
State of $\mathrm{W}_{\text {et }}$ & {$[1.1,2.2] / 26,(2.2,4.7] / 38,(4.7,9.3] / 53$} \\
\end{tabular}

\subsubsection{Training the $\mathrm{BN}$}

After the structure of the $\mathrm{BN}$ is determined and the input parameters are discretized, the training database can be employed to learn the parameters to compute the conditional probability between variables and to obtain the CPTs for each node. A maximum likelihood estimation approach, or a Bayesian estimation method can be employed to learn the parameters with a complete database. For an incomplete database in which information about some observations is missing, the Expectation Maximization (EM) algorithm is employed to find maximum likelihood estimates for a set of parameters. 
The EM algorithm can be described as follows (Nielsen and Jensen 2009):

Step 1: For a certain sample $D_{l}$ with missing data, we use $\theta$ to represent the model parameters. So, an initial $\theta^{0}$ is chosen randomly. Supposing that the $t$-th iteration has been completed, we get $\theta^{t}$.

Step 2: Expectation step

The aim of this step is to get a complete data set. To that end, we suppose that the set of missing values is $X_{l}$, so that the conditional probability distribution is calculated using Bayes theorem:

$$
P\left(X_{l} \mid D_{l}, \theta^{t}\right)=\frac{P\left(D_{l} \mid X_{l}, \theta^{t}\right) P\left(X_{l}, \theta^{t}\right)}{\sum_{X_{l}} P\left(D_{l} \mid X_{l}, \theta^{t}\right) P\left(X_{l}, \theta^{t}\right)}
$$

where $P\left(X_{l} \mid D_{l}, \theta^{t}\right)$ is called the weight of the fractional sample; it is related to the missing value $X_{l}$, which will be added to the incomplete data set to obtain the 'complete' data set.

\section{Step 3: Maximization step}

According to the new 'complete' data set, a MLE approach is employed to obtain $\theta^{t+1}$ Step 4: Comparing $\theta^{t+1}$ and $\theta^{t}$.

If $\theta$ obtained in Step 3 is converged, the algorithm ends. Else, we can return to step 1 , and make $\theta^{t}=\theta^{t+1}$ for another loop of calculation.

After the CPTs are obtained with the EM algorithm, the next step is to compute the posterior probability distribution given a set of observations (evidence). This process is referred to as belief updating (Korb and Nicholson 2010). The computation is usually carried out with the Junction Tree algorithm (Lauritzen and Spiegelhalter 1988). The 
main steps of the JT algorithm can be summarized as: construct a junction tree based on the exiting $\mathrm{BN}$; propagate messages along the junction tree using a message passing algorithm; answer queries when evidence is introduced. For a detailed introduction to the JT algorithm, the reader is referred to the work by Nielsen and Jensen (2009) and Korb and Nicholson (2010).

\subsection{Results and discussion}

\subsubsection{Development and validation of the $\mathrm{BN}$}

We use the software Netica to train the BN using the data set of case histories collected for this dissertation that was discussed in Chapter 2. The EM algorithm is applied to three structures of the $\mathrm{BN}$, in conjunction with the JT algorithm employed to propagate uncertainties, to "learn" the network parameters using the data set of the rock burst case histories. The result are shown in Figure 4.6. (In this analysis, we assume that no prior information is available, so that each state of the nodes in the network has an equal prior probability.)

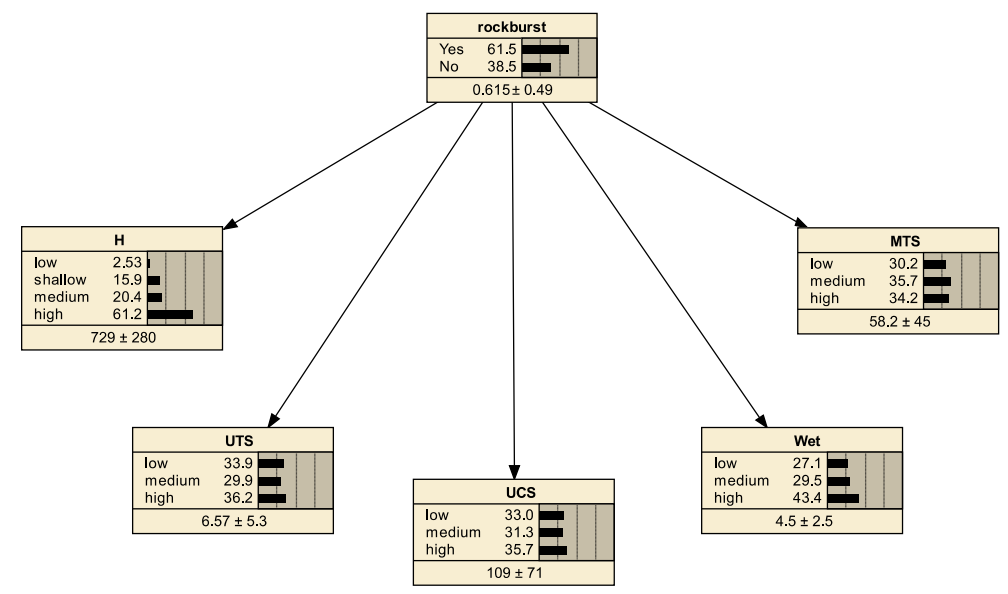

(a) NB classifier 


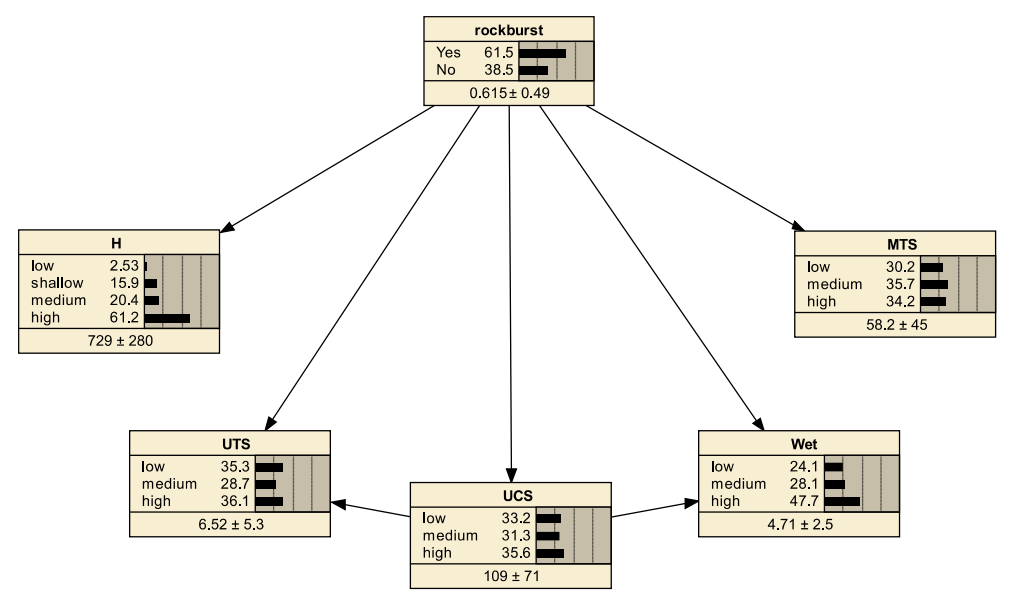

(b) TAN classifier

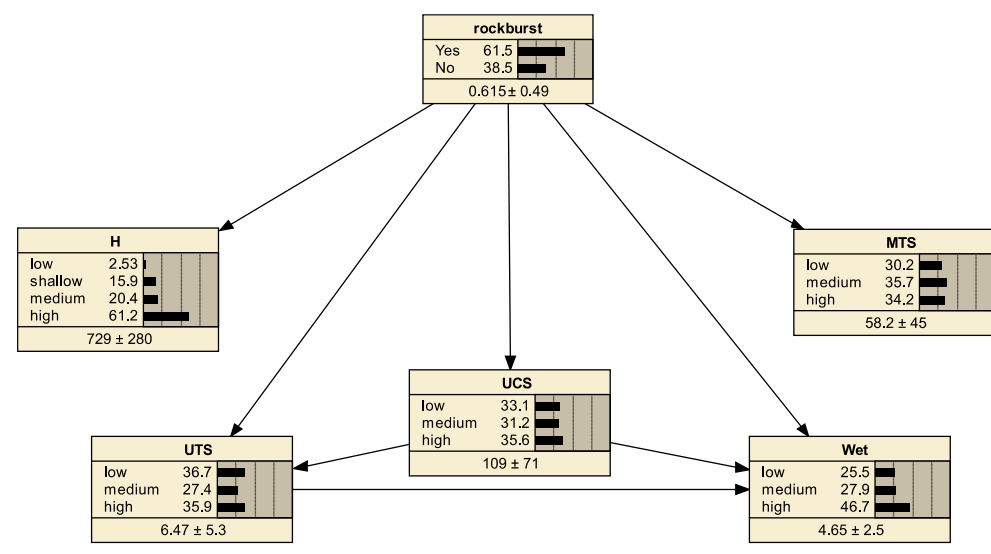

(c) BAN classifier

Figure 4.6 The BN after the EM algorithm is applied to the input parameters for training, assuming that no prior information is available

The confusion matrices for all the 135 case histories are presented in Table 4.2. The main diagonal represents the number of correct predictions, whereas the other diagonal shows the false-positives and false-negatives.

There are several metrics to evaluate the performance of machine learning algorithms, but the Accuracy and Cohen's Kappa values are the most commonly employed ones for classification problems. The Accuracy can be calculated from the confusion matrix: supposing that $m_{i j}$ is the element of the $i t h$ row and $j t h$ column in a confusion matrix with $n$ rows and columns, the Accuracy can be expressed as (Fawcett 2006): 


$$
A c=\left(\frac{\sum_{i=1}^{n} m_{i i}}{\sum_{i, j=1}^{n} m_{i j}}\right) \times 100 \%
$$

Table 4.2 Confusion matrices of node "Rock burst" using three different classifiers

\begin{tabular}{lllll}
\hline \multirow{2}{*}{ Classifier } & \multicolumn{2}{c}{ Predicted } & & \\
\cline { 2 - 4 } & Yes & No & Yes & Actual \\
Naïve Bayes & 78 & 5 & No & \\
TAN & 12 & 40 & Yes & Actual \\
\cline { 2 - 4 } & 80 & 3 & No & \\
BAN & 8 & 44 & Yes & \multirow{2}{*}{ Actual } \\
\cline { 2 - 4 } & 78 & 5 & No & \\
\hline
\end{tabular}

The Cohen's Kappa value (or coefficient) is another metric to evaluate the prediction results. It is a robust measure of the proportion of cases classified correctly after the probability of chance agreement has been considered and removed (Kuhn and Johnson 2013; Zhou et al. 2015). It can be defined as (Cohen 1960):

$$
\begin{gathered}
\kappa=\frac{A c-p_{e}}{1-p_{e}} \\
p_{e}=\frac{\sum_{i=1}^{n} m_{i+} \cdot m_{+i}}{\left(\sum_{i, j=1}^{n} m_{i j}\right)^{2}}
\end{gathered}
$$

where $m_{+i}$ and $\mathrm{m}_{i+}$ are the total cases of $i$ th columns and rows in the confusion matrix, $A c$ is the accuracy value, and $P_{e}$ is the hypothetical probability of chance agreement. Note that, if the prediction results are in complete agreement, then $\kappa$ equals 1 . And, if there is no agreement among the results, then there is a chance that the value of $\kappa$ is less than 0 . 
The predictive performances of the three structures as their corresponding Accuracy and Kappa values are listed in Table 4.3. It can be noted that the TAN classifier performs with a higher accuracy to predict the original 135 case histories; it is followed by Naïve Bayes and BAN structures. And the Kappa metric shows the same performance trend with values that are all above 0.4 , hence indicating a good agreement and reliable prediction (Landis and Koch 1977).

Compared with the TAN classifier, Naïve Bayes and BAN classifiers have more false positive cases, whereas the false negative cases are almost the same. The "moderate" to "strong" UCS-UTS and UCS-W $-\mathrm{W}_{\text {et }}$ relationships show an important role in the BN structure, and successfully capturing the dependence among these features could improve the prediction accuracy. However, the BAN classifier demonstrates a worse performance despite its more complex structure. The reason is probably that more complex structures require larger data sets for training but the data set employed herein is limited.

Table 4.3 Comparison of structures and influence on the predictive performance

\begin{tabular}{lll}
\hline BN Structure & Accuracy & Kappa \\
\hline Naïve Bayes & $87.4 \%$ & 0.727 \\
TAN & $91.9 \%$ & 0.825 \\
BAN & $85.9 \%$ & 0.693 \\
\hline
\end{tabular}

Additional advantages and limitations of the classifiers are summarized in Table 4.4. The TAN classifier has the best predictive ability, and it could be optimal for the rock burst prediction problem considered, as it shows a better predictive ability with an acceptable accuracy while still maintaining a quite simple structure.

As the TAN classifier is the optimal one for the rock burst prediction, the CPTs of the nodes are listed in Table 4.5. 
Table 4.4 Advantages and limitations of three classifiers

\begin{tabular}{lll}
\hline BN Structure & Advantages & Limitations \\
\hline Naïve Bayes & $\begin{array}{l}\text { Simple structure } \\
\text { Easy to construct } \\
\text { Efficient classification process } \\
\text { Relatively simple structure } \\
\text { Capture the dependency among } \\
\text { nodes }\end{array}$ & $\begin{array}{l}\text { Risk of overfitting given relatively } \\
\text { small data sets }\end{array}$ \\
TAN & $\begin{array}{l}\text { Capture the dependency among assumption } \\
\text { nodes more accurately }\end{array}$ & $\begin{array}{l}\text { Complex structure } \\
\text { Risk of overfitting given relatively } \\
\text { small data sets }\end{array}$ \\
\hline
\end{tabular}

After BNs are trained, additional belief updating can be conducted with the JT algorithm to update prediction so that they account for new observations about the input vector that become available. As an example, let us image that we get data from an underground excavation project, such as: $\mathrm{H}=700 \mathrm{~m}$ (Deep), MTS= 48.75 $\mathrm{MPa}$ (Medium), $\mathrm{UCS}=180 \mathrm{MPa}\left(\right.$ High), $\mathrm{UTS}=6.7 \mathrm{MPa}($ High$)$ and $\mathrm{W}_{\mathrm{et}}=5.5$ (High). Then, the probability of node "rock burst" can be updated conditioned on the above data, obtaining that $\mathrm{P}($ Rock burst $=$ Yes $\mid \mathrm{H}=$ Deep, MTS= Medium, UCS $=$ High, UTS= Medium, $\left.\mathrm{W}_{\mathrm{et}}=\mathrm{High}\right)=99.3 \%$, so that rock burst is very likely under these conditions. But BNs are also capable of dealing with incomplete data. For instance, if we have another project for which the $\mathrm{W}_{\text {et }}$ and MTS are not known, so that the only available data are that $\mathrm{H}=720 \mathrm{~m}, \mathrm{UCS}=88.8 \mathrm{MPa}$ and $\mathrm{UTS}=3.32 \mathrm{MPa}$, we could compute the probability of rock burst given such evidence as $\mathrm{P}$ (Rock burst= Yes $\mid \mathrm{H}=$ Very Deep, $\mathrm{UCS}=$ Medium, $\mathrm{UTS}=$ Medium $)=36.2 \%$, indicating that rock burst is significantly less likely under that quite different state of information. The trained BNs can also be applied to predict the rock burst hazard of the case histories used for training. (A 50\% probability threshold is employed to differentiate between rock burst and non-rock burst prediction.) 
Table 4.5 The CPTs for each node in the TAN based BN

Rock burst:

\begin{tabular}{lll}
\hline CPT for $\mathrm{P}($ Rock burst $)(\%)$ & & \\
\hline Rock burst & NO & YES \\
$\mathrm{P}($ Rock burst $)$ & 38.5 & 61.5 \\
\hline
\end{tabular}

H:

\begin{tabular}{lllll}
\hline \multicolumn{2}{l}{ CPT for $\mathrm{P}(H \mid$ Rock burst $)(\%)$} & & & \\
\hline Rock burst/H & Shallow & Medium & Deep & Very deep \\
\hline NO & 2.8 & 14.1 & 25.4 & 57.7 \\
YES & 2.1 & 18.8 & 12.5 & 66.6 \\
\hline
\end{tabular}

UCS:

\begin{tabular}{llll}
\hline CPT for $\mathrm{P}($ UCS $\mid$ Rock & burst $)(\%)$ & & \\
\hline Rock burst/UCS & Low & Medium & High \\
\hline NO & 57.2 & 27.4 & 15.4 \\
YES & 18.1 & 33.7 & 48.2 \\
\hline
\end{tabular}

MTS:

\begin{tabular}{|c|c|c|c|}
\hline \multicolumn{4}{|c|}{ CPT for $\mathrm{P}(M T S \mid$ Rock burst $)(\%)$} \\
\hline Rock burst/MTS & Low & Medium & High \\
\hline $\mathrm{NO}$ & 70.6 & 17.6 & 11.8 \\
\hline YES & 4.8 & 47.0 & 48.2 \\
\hline
\end{tabular}

UTS:

\begin{tabular}{lllll}
\hline \multicolumn{2}{l}{ CPT for P(UTS|Rock burst, UCS $)(\%)$} & & & Medium \\
\hline Rock burst & UCS & Low & 38.9 & 5.5 \\
\hline NO & Low & 55.6 & 64.3 & 7.1 \\
NO & Medium & 28.6 & 62.5 & 12.5 \\
NO & High & 25.0 & 20.0 & 13.3 \\
YES & Low & 66.7 & 21.4 & 32.2 \\
YES & Medium & 46.4 & 10.0 & 85.0 \\
YES & High & 5.0 & &
\end{tabular}

Wet:

\begin{tabular}{lllll}
\hline CPT for $\mathrm{P}\left(W_{\text {et }} \mid\right.$ Rock burst,UCS $)(\%)$ & & & Medium \\
\hline Rock burst & UCS & Low & 31.0 & 0.1 \\
\hline NO & Low & 68.9 & 19.1 & 38.3 \\
NO & Medium & 42.6 & 0 & 100 \\
NO & High & 0 & 66.7 & 13.3 \\
YES & Low & 20.0 & 32.1 & 60.8 \\
YES & Medium & 7.1 & 17.5 & 80.0 \\
YES & High & 2.5 & &
\end{tabular}


We can also compare the predictions of our method with those of other classic methods to predict rock burst reported in Table 1.1 in Chapter 1. (Note that most of the criteria are simple equations depending on two or three parameters.) The corresponding Accuracy values are listed in Table 4.6, which also includes the result of prediction conducted with our BN. (Note that, except for the BN approach, it is not always possible to use all case histories; this is an advantage of BNs since they can cope with incomplete cases.) The Russenes criterion provides good estimations which are similar to those of the $\mathrm{BN}$, although it is not applicable to all the projects since the information needed is not reported for all of them. In summary, our method not only has the best predictive capability (among those considered herein), but it can also be employed to all kinds of data sets (complete or incomplete).

Table 4.6 Comparison of Accuracy values with four different methods and our BNs

\begin{tabular}{llll}
\hline Method & Unavailable cases & Available cases & Accuracy value \\
\hline $\begin{array}{l}\text { Russenes criterion } \\
\text { (Russenes 1974) }\end{array}$ & 35 & 100 & $90 \%$ \\
$\begin{array}{l}\text { Hoek criterion } \\
\text { (Hoek and Brown 1980) }\end{array}$ & 35 & 100 & $86 \%$ \\
$\begin{array}{l}\text { Rock brittleness coefficient } \\
\text { (Wang et al. 1998) }\end{array}$ & 12 & 123 & $66.6 \%$ \\
$\begin{array}{l}\text { Elastic energy index } \\
\text { (Wang et al. 1998) }\end{array}$ & 18 & 117 & $84.6 \%$ \\
BNs with all data & 0 & 135 & $91.85 \%$ \\
\hline
\end{tabular}

A k-fold cross-validation can be further employed to validate the model and to test the accuracy of the $\mathrm{BN}$. To that end, we can divide the 135 case histories available in the initial database into 8 groups. For each group, the $\mathrm{BN}$ is trained using the other 7 groups, and the originally selected group is then used to predict rock burst occurrence with the trained $\mathrm{BN}$, and to compare observations and predictions. If this process is repeated to all groups of data, an 8-fold cross-validation exercise is obtained. Results are reported 
in Table 4.7, where it can also be noted that most error cases are false-positives, hence suggesting a conservative prediction, which is much safer for actual engineering.

An additional validation can be conducted employing new case histories. Therefore, another 15 new case histories with missing data are collected from the literature. (Some cases are from tunnels, and some are from metal and coal mines.) Their observed rock burst occurrence (or not), as well as the prediction obtained with the $\mathrm{BN}$ and other four methods in Table 1.1, are reported in Table 4.8, where one can observe that the BN performed very well for most cases, with only one wrong prediction. 
Table 4.7 The confusion matrices of the 8 groups of data employed by the crossvalidation (The average Accuracy value is $85.21 \%$ )

\begin{tabular}{|c|c|c|c|}
\hline \multicolumn{4}{|c|}{ Confusion matrices } \\
\hline \multicolumn{2}{|c|}{ Predicted } & & \\
\hline Yes & No & & \\
\hline 11 & 0 & Yes & Actual \\
\hline 4 & 2 & No & \\
\hline \multicolumn{2}{|c|}{ Predicted } & & \\
\hline Yes & No & & Actual \\
\hline 10 & 3 & Yes & \\
\hline 1 & 3 & No & \\
\hline \multicolumn{2}{|c|}{ Predicted } & & \\
\hline Yes & No & & Actual \\
\hline 10 & 0 & Yes & \\
\hline 0 & 7 & No & \\
\hline \multicolumn{2}{|c|}{ Predicted } & & \\
\hline Yes & No & & Actual \\
\hline 7 & 1 & Yes & \\
\hline 1 & 8 & No & \\
\hline \multicolumn{2}{|c|}{ Predicted } & & \\
\hline Yes & No & & Actual \\
\hline 9 & 2 & Yes & \\
\hline 0 & 6 & No & \\
\hline \multicolumn{2}{|c|}{ Predicted } & & \\
\hline Yes & No & & Actual \\
\hline 10 & 0 & Yes & \\
\hline 2 & 5 & No & \\
\hline \multicolumn{2}{|c|}{ Predicted } & & \\
\hline Yes & No & & Actual \\
\hline 10 & 1 & Yes & \\
\hline 3 & 3 & No & \\
\hline \multicolumn{2}{|c|}{ Predicted } & & \\
\hline Yes & No & & Actual \\
\hline 9 & 0 & Yes & \\
\hline 2 & 5 & No & \\
\hline
\end{tabular}




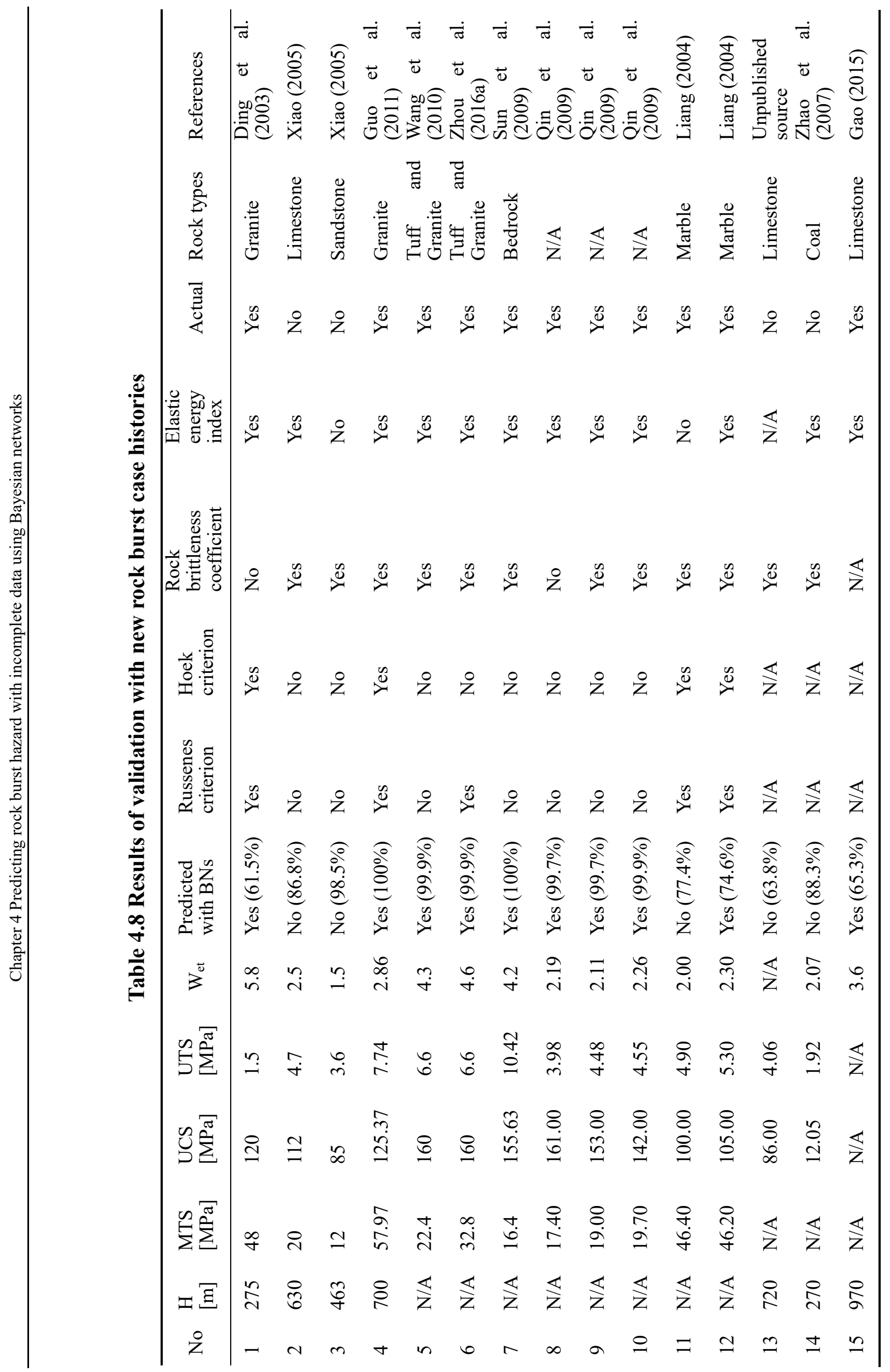




\subsubsection{Sensitivity analyses}

An additional useful feature of BNs is that they can inform about the influence of parameters on rock burst prediction. To that end, two common methods - the mutual information method and the variance reduction method - are employed to determine the input nodes with influence on the target node. The Mutual information method was proposed by Shannon and Weaver (1998): for two discrete variables $X_{\text {tar }}$ (the target node) and $X_{\text {in }}$ (the input node), the mutual information between them can be expressed as

$$
I\left(X_{t a r}, X_{i n}\right)=\sum_{x_{t a r}, x_{i n}} P\left(x_{t a r}, x_{i n}\right) \ln \frac{P\left(x_{t a r}, x_{i n}\right)}{P\left(x_{t a r}\right) P\left(x_{i n}\right)}
$$

The mutual information equals 0 when $X_{\text {tar }}$ is independent of $X_{i n}$.

The variance reduction method was proposed based on cost-benefit considerations (Pearl 2014). It can be expressed as

$$
\operatorname{Vr}\left(X_{\text {tar }}, X_{\text {in }}\right)=\sum_{x_{i n}} P\left(x_{i n}\right) \sum_{x_{\text {tar }}} P\left(x_{t a r} \mid x_{i n}\right)\left(x_{t a r}-\mu_{x_{\text {tar }} \mid x_{\text {in }}}\right)^{2}
$$

where $\mu_{x_{\text {tar }} x_{\text {in }}}=\sum_{x_{\text {tar }}} P\left(x_{\text {tar }} \mid x_{\text {in }}\right) x_{\text {tar }}$. The variance reduction is also equal to 0 when $X_{t a r}$ is independent of $X_{i n}$.

Table 4.9 reports the sensitivities of the five parameters considered in relation to rock burst occurrence. (Results are computed by Netica.) The two percent columns represent the percent contribution of each node to the "rock burst" node, showing that the maximum tangential stress has the highest percent contribution, being therefore the most influential parameter on rock burst occurrence. (Note that, perhaps surprisingly, the MTS is often ignored during rock burst prediction, whereas the UCS and $\mathrm{W}_{\text {et }}$ parameters, which seem to be less influential, are quite commonly employed for such task.) Similarly, although the buried depth factor is considered as an important factor in many studies, our results suggest that it might be not so important, in agreement with 
the observation that rock burst accidents also happen in shallow tunnels or shallow mining roadways.

Table 4.9 Results of sensitivity analyses for node "Rock burst" in the BN model

\begin{tabular}{lllll}
\hline Node & $\begin{array}{l}\text { Variance } \\
\text { Reduction }\end{array}$ & Percent [\%] & $\begin{array}{l}\text { Mutual } \\
\text { information }\end{array}$ & Percent [\%] \\
\hline Rock burst & 0.2368 & 100 & 0.96162 & 100 \\
H & 0.006158 & 2.6 & 0.01969 & 2.05 \\
MTS & 0.1158 & 48.9 & 0.37829 & 39.3 \\
UCS & 0.04367 & 18.4 & 0.13704 & 14.3 \\
UTS & 0.05978 & 25.2 & 0.20646 & 21.5 \\
W & 0.06106 & 25.8 & 0.18981 & 19.7 \\
\hline
\end{tabular}

\subsection{Summary}

A novel application of Bayesian networks to predict rock burst occurrence is presented. The Naïve Bayes, Tree augmented Naïve Bayes and BN augmented Naïve Bayes classifiers are proposed to predict the probability of rock burst given information on five parameters — buried depth of the tunnel, maximum tangential stress of surrounding rock, uniaxial tensile strength of rock, uniaxial compressive strength of rock and elastic energy index - that we employ as inputs, and based on which the predictions are obtained. The $\mathrm{BN}$ is trained with the Expectation Maximization algorithm, using the 135 case histories included in the database that we compiled from the literature; and the Junction Tree algorithm is employed for Belief updating when new information is available or to make predictions based on information about the input parameters. The results show that the Tree augmented Naïve Bayes classifier is the optimal classifier for the rock burst prediction work.

The trained $\mathrm{BN}$ has been validated using cross-validation and the original database. In addition, 15 new cases with incomplete information are employed to test the 
performance of the BN's prediction. Results show that the Accuracy value on rock burst prediction of the trained $\mathrm{BN}$ is among the highest of the commonly employed methods considered; and that it is probably high enough so as to be acceptable for practical engineering. This suggests that the $\mathrm{BN}$ methods provide useful information about the likelihood of rock burst during tunneling or mining processes. The proposed $\mathrm{BN}$ has the additional advantage of being able to deal with, and to make predictions in situations in which, as it often happen in practice, information about some of the parameters is not available.

In addition, sensitivity analyses are conducted to identify the input parameters with a stronger influence on rock burst. Results suggest that the maximum tangential stress is the most influential parameter, with other factors such as UCS and UTS having also a large influence on rock burst prediction. Other factors, such as the buried depth, have only a secondary influence on the rock burst outcome, and could probably be considered as external reference factors. It is expected that these results can lead designers to focus their characterization efforts forward those factors that have been identified as more relevant. 



\section{EVALUATING SHORT-}

\section{TERM ROCK BURST}

\section{DAMAGE USING ROCK}

\section{ENGINEERING SYSTEMS}

\subsection{Introduction}

Short-term prediction of rock burst aims to predict the location, time and damage scale of rock burst occurrence based on the dynamic and continuous analyses of data on site. This field has affected a lot of attention in recent years. As discussed in Chapter 1, several approaches have been proposed to try to anticipate rock burst occurrence in the short term. However, most of these works cannot be easily applied for prediction. In addition, a clear understanding on the relationships among the parameters that affect rock burst is lacking, as this problem has not been previously addressed considering a wide system perspective. 
Therefore, herein we propose a novel approach based on the combined use of the Rock Engineering Systems (RES) and of machine learning methods to develop a simple and easily applicable index — the rock burst damage index, or RDSI — to analyze the problem of short-term rock burst occurrence, with its associated consequences.

\subsection{Description of parameters and of the prediction system}

Previously proposed methods for long-term rock burst prediction (see Chapters 3 and 4) only considered five main parameters $-\mathrm{H}$, UCS, UTS, MTS and $\mathrm{W}_{\mathrm{et}}-$, or their combination (Zhou et al. 2015; Zhou et al. 2016a). However, as those parameters are mainly selected due to their availability at early stages of a project — hence being mainly useful for long-term prediction - , they might be inadequate (or insufficient) of short-term prediction. In other words, the analyses of short-term rock burst occurrence should incorporate additional information about the tunnel and its construction, such as the daily advance of the tunnel or working face, the actual geological structures encountered, or the installed support measures.

\subsubsection{Description of selected parameters}

The eight parameters employed for our analyses of rock burst prediction are discussed next.

\subsubsection{Stress condition $(\mathrm{P} 1)$}

The ratio of total maximum principal stress to the uniaxial compressive strength of the rock $\left(\sigma_{1} / \mathrm{UCS}\right)$ could affect the rock burst damage on site, and many scholars have employed this parameter for rock burst hazard evaluation (see e.g., Li et al. (2012)). For instance, Kaiser and Maloney (1997) proposed an index that used this $\sigma_{1} / \mathrm{UCS}$ ratio in the region of the opening to assess the potential rock burst hazard. 


\subsubsection{Support condition (P2)}

Ground control measures and burst-resistant rock support are used to prevent or minimize damage to excavations and thus to enhance workplace safety (Kaiser and Cai 2012). An integrated rock support system against rock burst combines reinforcement, retaining and holding, and seven principles regarded to rock support design in burstprone ground were proposed by Cai (2013). The main idea is that an optimal system should be strong enough to transfer static loads to rock bolts and cables, making them able to resist tremors induced by the dynamic loads. In our database, the capacity of the ground support system can be estimated by the types of support system employed: 49 different systems have been identified, and they can be discretized for our prediction using the rating criteria proposed by Heal (2010).

\subsubsection{Excavation environment (P3)}

The excavation span - as defined for instance following the recommendation by Zhou et al. (2016c), who consider the width of the underground opening in plan view- has a direct influence on rock seam or roof collapse, on ground surface subsidence and on dynamic rock mass failure or rock burst (Heal 2010). The supporting system or rock pillar should be therefore reinforced to ensure the stability of the tunnel when a larger excavation span is employed. Therefore, the excavation span is used herein to reflect the actual excavation environment.

\subsubsection{Geological parameter (P4)}

Geological conditions (faults, folds and other underground structures) are important factors that should be considered in the short-term prediction of rock burst, as they can affect the stress distribution and cause stress concentrations that may result in rock burst 
(Dou et al. 2006; Li et al. 2014). For simplicity, the geological data is discretized into three categories in the database. The consideration of these categories adequately captures the variability in the case histories (removing subjectivity while maintaining simplicity), and it also avoids the use of a more detailed structural classification system (Heal 2010).

\subsubsection{Seismic related data (P5 and P7)}

As the installation of micro seismic monitoring systems in mines has become increasingly common, many seismic related data have been recorded and become available to be analyzed for rock burst prediction. Kaiser et al. (1996) employed peak particle velocity (PPV) to predict rock burst hazard and illustrated that the potential and type of hazard changes with the increase of the PPV values. Alcott et al. (1998) assessed rock burst hazard using micro seismic source parameters, like seismic event magnitude or moment and peak particle velocity. Similarly, rock burst frequency and magnitude using micro seismic data in coal mines of China have been studied by Dou et al. (2006). Kaiser and Maloney (1997) analyzed the relationship between PPV and the seismic energy of rock burst events; whereas Kaiser (as cited by Heal (2010)) proposed a relationship between PPV and the Richter magnitude of such events.

Therefore, the PPV and other seismic magnitude-related parameters can be chosen to predict the damage due to the rock burst occurrence.

\subsubsection{Rock mass density (P9)}

Although such rock density in real application often has a limited range of variability that makes it to be often assumed as constant in practical applications (Dwivedi et al. 2013), the database developed in this work compiles data from 13 mines with quite 
different density records. For that reason, and because rock density also affects the relationship between dynamic loading (dynamic stress) and PPV (Kaiser et al. 1996), we decided to use it for our prediction work. The database compiled contains 7 density values, and the parameter is discretized according to the range of density values.

\subsubsection{Rock burst damage scale (RDS) (P14)}

Five levels are considered for rock burst damage. The qualification of the scale is based on the quantity of rock ejected when rock burst occurs (Kaiser et al. 1992).

The five levels considered are:

R1: no damage and minor loose of the rock mass;

$\mathrm{R} 2$ : less than $1 \mathrm{t}$ of rock displaced with minor damage;

R3: 1 to 10 t of rock displaced;

R4: 10 to 100 t of rock displaced;

R5: above $100 \mathrm{t}$ of rock displaced.

The reader should note, however, that occurrence of the R1 scale of damage is often not reported in case histories, as damage report sheets are only prepared when actual damage occurs. But this should not affect our analyses, as the aim of this study is to estimate the damage condition associated to rock burst hazards in underground construction, hence being mainly concerned with cases with different levels of damage. And, of course, additional information about rock burst cases with no damage could be employed to improve the predictive models when such information becomes available. 
Table 5.1 Classification and discretization values for all the parameters

\begin{tabular}{|c|c|c|c|c|c|c|}
\hline \multirow{2}{*}{$\begin{array}{l}\text { Parameters } \\
\text { Stress condition } \\
\sigma_{1} / \mathrm{UCS}\end{array}$} & \multicolumn{6}{|c|}{ Classification and discretization values } \\
\hline & $<39.2$ & 39. & -48.65 & $48.65-58.95$ & $\begin{array}{l}58.95- \\
70.65\end{array}$ & $>70.65$ \\
\hline Normalized value & 0 & 0.2 & & 0.5 & 0.75 & 1 \\
\hline Support condition & $\begin{array}{l}\text { Dynamic } \\
\text { support } \\
\text { with pattern } \\
\text { dynamic } \\
\text { reinforce- } \\
\text { ment }\end{array}$ & $\begin{array}{l}\text { Me } \\
\text { fibi } \\
\text { sup } \\
\text { pat } \\
\text { and } \\
\text { bol }\end{array}$ & $\begin{array}{l}\mathrm{h} \text { or } \\
\text { crete } \\
\text { ort with } \\
\text { rn bolting } \\
\text { cable }\end{array}$ & $\begin{array}{l}\text { Mesh or } \\
\text { fibrecrete } \\
\text { support with } \\
\text { pattern } \\
\text { bolting and a } \\
\text { second } \\
\text { bolting }\end{array}$ & $\begin{array}{l}\text { Mesh or } \\
\text { fibrecrete } \\
\text { support } \\
\text { with } \\
\text { pattern } \\
\text { bolting }\end{array}$ & $\begin{array}{l}\text { No surface } \\
\text { support } \\
\text { with spot } \\
\text { bolting } \\
\text { reinforce- } \\
\text { ment }\end{array}$ \\
\hline Normalized value & 0 & 0.2 & & 0.5 & 0.75 & 1 \\
\hline $\begin{array}{l}\text { Excavation span } \\
{[\mathrm{m}]}\end{array}$ & $<5.15$ & 5.1 & -5.95 & $5.95-7.05$ & $7.05-9.45$ & $>9.45$ \\
\hline Normalized value & 0 & 0.2 & & 0.5 & 0.75 & 1 \\
\hline Geological factor & $\begin{array}{l}\text { No major } \\
\text { structures lik } \\
\text { faults, folds, } \\
\text { shears exits, } \\
\text { minor blast } \\
\text { related struct }\end{array}$ & & $\begin{array}{l}\text { No major } \\
\text { faults, fol } \\
\text { exits, but } \\
\text { discontinu } \\
\text { unstable r } \\
\text { rock mass } \\
\text { rock failur }\end{array}$ & $\begin{array}{l}\text { tructures like } \\
\text { s, or shear } \\
\text { he } \\
\text { ity and } \\
\text { ck block in } \\
\text { may promote } \\
\text { e }\end{array}$ & $\begin{array}{l}\text { Major structu } \\
\text { faults, folds, } \\
\text { that could ind } \\
\text { burst occurre }\end{array}$ & $\begin{array}{l}\text { res, like } \\
\text { shears exits, } \\
\text { luce the rock } \\
\text { nce }\end{array}$ \\
\hline Normalized value & 0 & & 0.5 & & 1 & \\
\hline $\begin{array}{l}\text { Seismic event } \\
\text { magnitude }\end{array}$ & $<1.25$ & 1.2 & -1.65 & $1.65-2.05$ & $2.05-2.6$ & $>2.6$ \\
\hline Normalized value & 0 & 0.2 & & 0.5 & 0.75 & 1 \\
\hline $\begin{array}{lr}\text { Particle } & \text { peak } \\
\text { velocity } & (\mathrm{PPV}) \\
{[\mathrm{m} / \mathrm{s}]} & \end{array}$ & $<0.575$ & 0.5 & $5-1.02$ & $1.02-1.71$ & $1.71-2.71$ & $>2.71$ \\
\hline Normalized value & 0 & 0.2 & & 0.5 & 0.75 & 1 \\
\hline $\begin{array}{l}\text { Rock mass density } \\
{\left[\mathrm{kg} / \mathrm{m}^{3}\right]}\end{array}$ & $<2800$ & & -2900 & $2900-3000$ & $3000-3100$ & $>3100$ \\
\hline Normalized value & 0 & 0.2 & & 0.4 & 0.6 & 1 \\
\hline
\end{tabular}

Table 5.1 shows a summary of all values employed for classification and discretization of parameters. To that end, the geological and support condition parameters in the original database have been discretized using the reference values proposed by Heal (2010); whereas the software WEKA has been employed to conduct the discretization of the other parameters.

\subsubsection{Rock burst damage prediction system}


Considering all the parameters employed, a rock burst damage prediction system can be established intuitively, as shown in Figure 5.1. The flowchart shows that our employed parameters include three main factors that could affect rock burst occurrence, like support condition, static loading and dynamic loading. Using such factors, with the complement of excavation and geological factors, a rock burst damage scale index can be developed systematically, as explained in the following section.

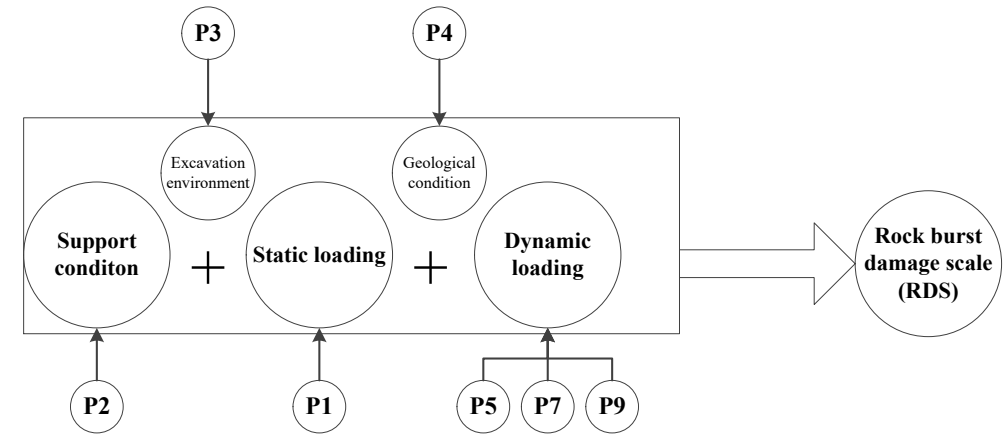

Figure 5.1 Rock burst damage prediction system

\subsection{The Rock Engineering Systems (RES) approach}

\subsubsection{Introduction}

The Rock Engineering Systems (RES) approach proposed by Hudson (1992) is a powerful tool to deal with complicated rock engineering problems due to its adaptability, comprehensiveness, repeatability, efficiency and effectiveness (Hudson and Harrison 1992). This approach has been applied in slope stability, surface and underground blasting, road side slop failure, TBM performance and support in tunnel and roadways, among other applications (Naghadehi et al. 2011; Hudson 2013; Naghadehi et al. 2013).

\subsubsection{Interrelations of factors}

The key for RES analyses is to establish the interaction matrix that reflects the mutual interrelations between all the factors considered. Figure 5.2 shows a conceptual 
example of a two parameter interrelation matrix, and Figure 5.3 describes a specific example considering "rock discontinuity" and "rock stress" as factors affecting the system's behavior. The influence of each individual factor on the other factor is arranged at the off-diagonal positions. As the interaction values can be different depending on the type of relationship (i.e., the influence of $\mathrm{x}$ on $\mathrm{y}$ can be different to the influence of $y$ on $x$ ), the matrix is not symmetric.

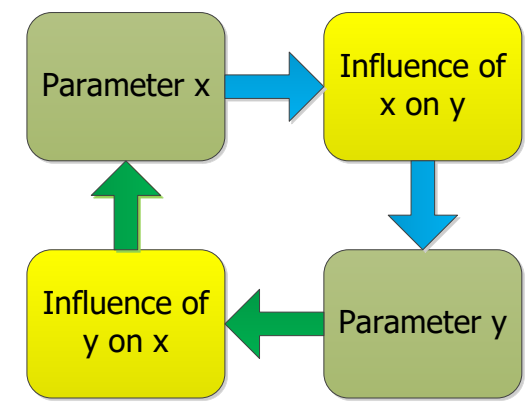

Figure 5.2 Interaction matrix with factors $x$ and $y$

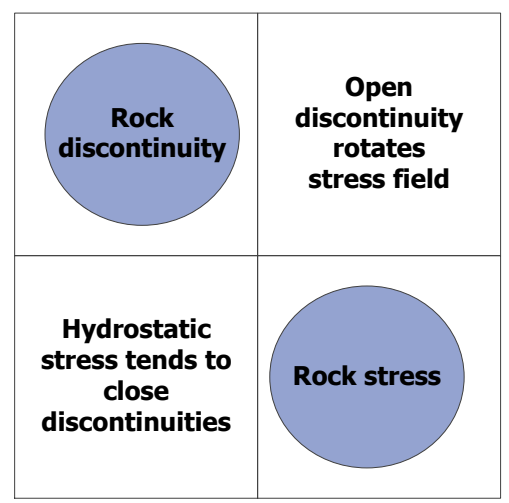

Figure 5.3 A 2×2 interaction matrix with leading diagonal terms, rock discontinuity and rock stress (based on Hudson (1992))

\subsubsection{Coding the matrix}

There are several methods to decide the influence of one factor on the other factors, in a process which is normally referred to as "coding the matrix". The most common methods are the binary method and the expert semi-quantitative method.

The binary method uses expert opinion to assign one of two values ( 0 or 1$)$ to the 
interaction, hence producing a binary interaction matrix (BIM). (Note that " 1 " means that an interaction relationship exists and " 0 " means that there is no relationship between the parameters.) The expert semi-quantitative method uses a wide range of interaction values; a 5-level (from 0 to 4) description of interaction is commonly employed, where " 0 " means no interaction; "1" means weak interaction; " 2 " means medium interaction; "3" means strong interaction; and "4" means critical interaction. However, these two methods have the shortcoming that the assigned values are often subjective, and that it is often difficult to assign a unique and constant interaction value.

To overcome these problems, a probabilistic coding methodology was proposed by Naghadehi et al. (2011), in which the likelihood of each possible coding value (considered from 0 to 4 ) is assigned probabilistically. This provides a more reliable matrix, although it is still somewhat subjective, due to it being produced with the aid of "expert-opinion". The matrix can also be coded automatically based on the available data using Artificial Neural Networks, which are employed to analyze the interaction. As previous examples of such methodologies in the geotechnical field, see the work of Naghadehi et al. (2013) on open-pit mine slope stability applications. In this dissertation, we use such approach and, in particular, we employ the ANN to develop the interaction matrix between the factors employed for the short-term prediction of rock burst damage in underground excavations.

\subsubsection{Matrix analyses}

Once the coding process is completed, one can compute the sum of each row and column in the matrix. For each parameter, the sum of interaction within each row is termed as the "cause" (C), and the sum of interaction within each column is termed as the "effect" (E). Figure 5.4 shows a diagram of a basic interaction matrix and related C 
and E. Mathematically, for the $i$-th parameter, we have:

$$
\begin{aligned}
& C_{i}=\sum_{j \neq i}^{m} I_{i j} \\
& E_{i}=\sum_{k \neq i}^{m} I_{k i}
\end{aligned}
$$

where $m$ is the number of parameters, $I_{i j}$ is the influence of parameter $i$ on parameter $j$. Such computed $C_{i}$ and $E_{i}$ values can be regarded as the coordinates $\left(C_{i}, E_{i}\right)$ for parameter $i$, so that the points corresponding to all parameters can be represented in the Cause-Effect plot (see Figure 5.5). Such cause-effect plot illustrates whether the parameter is "dominant", affecting the rest of the system; or whether the system is "dominant".

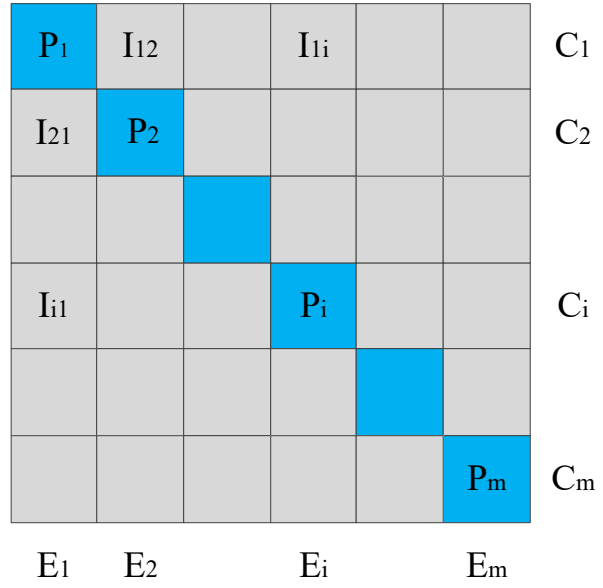

Figure 5.4 Interaction matrix and related C and E (based on Hudson (1992))

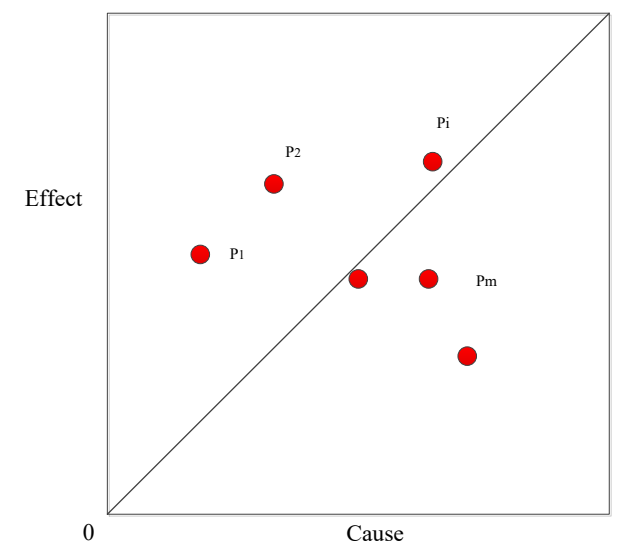

Figure 5.5 The (C, E) plot with $m$ influencing factors (based on Hudson (1992)) 


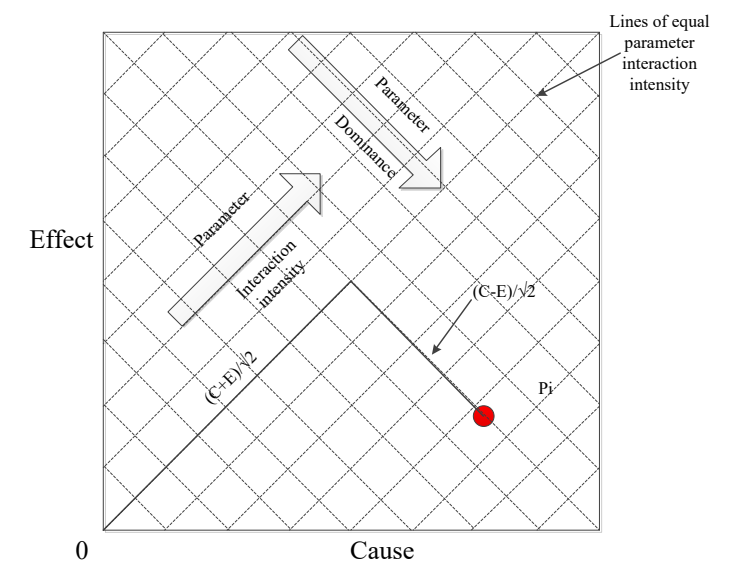

\section{Figure 5.6 The (C, E) plot with lines of equal parameter interaction intensity and dominance (based on Hudson (1992))}

The parameter interaction intensity and the parameter dominance are graphically shown in the Cause-Effect plot of Figure 5.6. The parameter interaction intensity can be measured as $(\mathrm{C}+\mathrm{E}) / \sqrt{ } 2$ which is the distance from the origin to the foot of perpendicular line from the parameter's $(\mathrm{C}, \mathrm{E})$ coordinates to the $\mathrm{C}=\mathrm{E}$ line, whereas the parameter dominance can be measured as $(C-E) / \sqrt{ } 2$ which is the perpendicular distance from the parameter's (C, E) coordinates to the $\mathrm{C}=\mathrm{E}$ line (Hudson 1992).

\subsubsection{Application on rock burst damage}

The interrelations between influencing parameters are often omitted in rock burst prediction analyses, and only the influence or the sensitivity of each parameter on the output targets is considered. Therefore, in order to analyze mutual information among the employed parameters that could affect the RDS estimation, the RES approach is employed here, together with Artificial Neural Networks (ANN) employed to code the matrix coding method and to analyze the interaction relationships. The matrix with 7 parameters for RDS prediction is shown in Figure 5.7. 


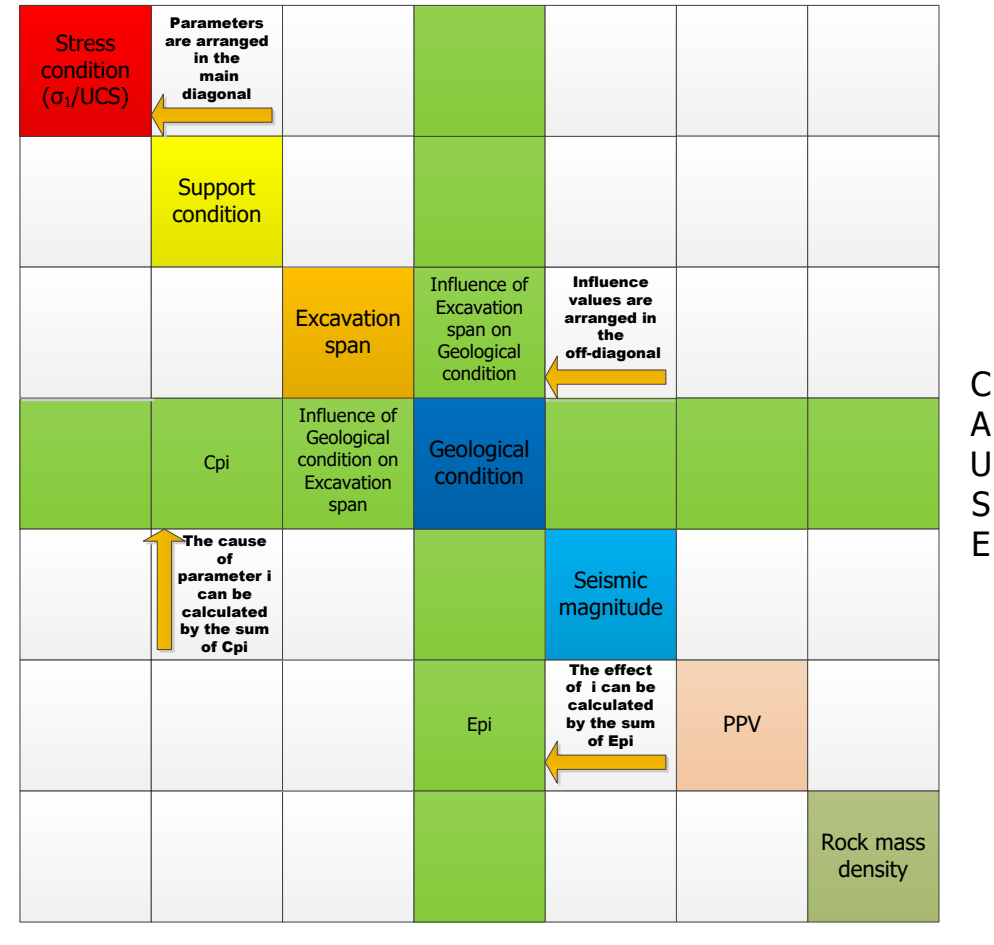

EFFECT

Figure 5.7 The RES matrix for RDS prediction

The cause (C) and effect (E) can be calculated by each parameters' coding values.

The influence weights of parameters are defined as follows:

$$
a_{i}=\left(\frac{C_{i}+E_{i}}{\sum_{i}^{m}\left(C_{i}+E_{i}\right)}\right) \times 100
$$

We suppose that the weights of all the parameters is listed in a vector, $A$, with $A=\left(a_{1}\right.$, $\left.a_{2}, a_{3}, a_{4}, a_{5}, a_{6}, a_{7}\right)$. For a case history $k$, all the 7 parameters can be normalized according to the classification intervals in Table 5.1, providing a vector $X_{k}$, with $X_{k}=\left(x_{1 \mathrm{k}}\right.$, $x_{2 \mathrm{k}}, x_{3 \mathrm{k}}, x_{4 \mathrm{k}}, x_{5 \mathrm{k}}, x_{6 \mathrm{k}}, x_{7 \mathrm{k}}$ ), so that the index of rock burst damage scale (RDSI) can be expressed as:

$$
R D S I=X_{k} \cdot A^{T}
$$




\subsection{Model training}

The application of ANN to develop the interaction matrix of RES analyses was proposed by Yang and Zhang (1998). The idea is that, since ANN can solve complicated mappings from input to output, they can be a powerful tool to analyze the interactions of parameters in RES analyses. However, the training of the network requires a large set of data, so that the collected database of case histories is applied to obtain the global relative strength effects (GRSE) defined below.

\subsubsection{Structure of the ANN}

With its simplicity and applicability, the standard Backpropagation (BP) algorithm is employed for ANN training. The sigmoid activation function is used for each layer of the network with an output range from 0 to 1 . Figure 5.8 shows the structure of a common network, with the input layer $O_{i}$, output layer $O_{k}$ and hidden layers $O_{j 1}$ to $O_{j k}$. A bias $\left(b_{i}, b_{j l}, \ldots, b_{j k}\right)$ is also included in each layer to adjust the activation function for a successful learning.

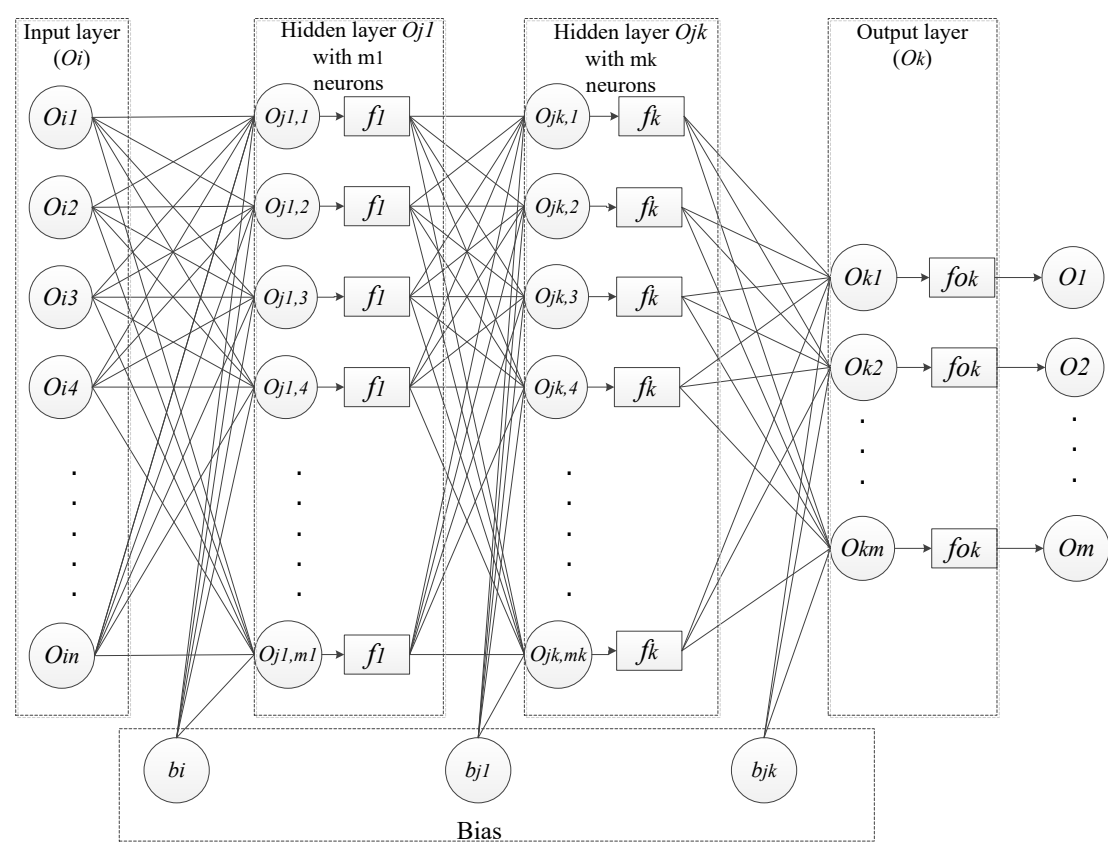

Figure 5.8 The structure of common ANN 
The BP algorithm repeats a two phase cycle — propagation and weight updatingwhich can be described as follows (Li et al. 2009):

Phase 1: Forward pass (Propagation)

In the forward pass, the output nodes are obtained from the input nodes through the neural network using the weight and bias. The delta (or errors) can be calculated using the difference between the computed and observed output values.

Phase 2: Backward pass (Weight update)

In the backward pass, the weights in the network are updated so that they cause the actual output to be closer to the observed (or target) output. To that end, the derivative of delta to the related weight can be computed to find the gradient of the weight. Then, the weight of each nodes and layers are updated in the opposite direction using the gradient descent algorithm.

Phases 1 and 2 are repeated until the performance of the network is satisfactory.

\subsubsection{Network training and GRSE}

The global relative strength of effect (GRSE) was proposed by Yang and Zhang (1998) and has been employed for the analyses of open-pit mine slope instability conducted by Naghadehi et al. (2013). A brief introduction of the GRSE is discussed next, and detailed analyses can be seen in Yang and Zhang (1998) and Naghadehi et al. (2013).

In order to obtain the interaction relationship of the parameters, the partial derivative of the output parameter to the input parameter is calculated with the functions considered in each hidden and final output layers: 


$$
\begin{aligned}
& \frac{\partial O_{m}}{\partial O i}=\sum_{m k}\left(\frac{\partial f_{o k}\left(O_{k}\right)}{\partial f_{k}\left(O_{j k}\right)}\right)\left(\frac{\partial f_{k}\left(O_{j k}\right)}{\partial f_{k-1}\left(O_{j k-1}\right)}\right) \cdots \sum_{m 1}\left(\frac{\partial f_{2}\left(O_{j 2}\right)}{\partial f_{1}\left(O_{j 1}\right)}\right)\left(\frac{\partial f_{1}\left(O_{j 1}\right)}{\partial O_{i}}\right) \\
& =\sum_{m k}\left(\frac{\partial f_{o k}\left(O_{k}\right)}{O_{k}} \frac{O_{k}}{\partial f_{k}\left(O_{j k}\right)}\right)\left(\frac{\partial f_{k}\left(O_{j k}\right)}{O_{j k}} \frac{O_{j k}}{\partial f_{k-1}\left(O_{j k-1}\right)}\right) \\
& \cdots \sum_{m 1}\left(\frac{\partial f_{2}\left(O_{j 2}\right)}{O_{j 2}} \frac{O_{j 2}}{\partial f_{1}\left(O_{j 1}\right)}\right)\left(\frac{\partial f_{1}\left(O_{j 1}\right)}{\partial O_{i}}\right)
\end{aligned}
$$

where

$$
\begin{gathered}
O_{j 1}=\mathrm{O}_{i} \mathrm{~W}_{i, j 1}+b i, f_{1}\left(O_{j 1}\right)=\frac{1}{\left(1+\exp \left(-\left(\mathrm{O}_{i} \mathrm{~W}_{i, j 1}+b i\right)\right)\right.} \\
O_{k}=\mathrm{O}_{j k} \mathrm{~W}_{j k, k}+b_{j k}, O_{m}=f_{o k}\left(O_{k}\right)=\frac{1}{\left(1+\exp \left(-\left(\mathrm{O}_{j k} \mathrm{~W}_{j k, k}+b_{j k}\right)\right)\right.}
\end{gathered}
$$

As $\frac{\partial f_{o k}\left(O_{k}\right)}{O_{k}}=D_{o k}, \frac{O_{k}}{f_{k}\left(O_{j k}\right)}=W_{j k, k}$, we can have the following equation:

$$
\frac{\partial O_{m}}{\partial O_{i}}=\sum_{m k} \sum_{m k-1} \cdots \sum_{m 1} D_{o k} W_{j k, k} D_{j k} W_{j k-1, j k} \cdots D_{j 2} W_{j 1, j 2} W_{i, j 1}
$$

The weight $(\mathrm{W})$ between each layer can be obtained and fixed from the network training. As our input parameters are in the range of 0 to 1 , the relative strength of effect can be defined from the above equation as:

$$
R S E_{m i}=C \sum_{m k} \sum_{m k-1} \cdots \sum_{m 1} D_{o k} W_{j k, k} D_{j k} W_{j k-1, j k} \cdots D_{j 2} W_{j 1, j 2} W_{i, j 1}
$$

where $C$ is a normalizing constant to limit the maximum absolute value of $R S E_{m i}$ to 1 .

This relative strength can be seen as the fully coupled relative influence of one given input on one given output when all the connections between the two parameters are considered.

However, the above RSE values always change with the position of the inputs, so that the global relative strength effect (GRSE) is proposed, considering that the derivative of the activation function is constant. It can be expressed as:

$$
G R S E_{m i}=C \sum_{m k} \sum_{m k-1} \cdots \sum_{m 1} W_{j k, k} W_{j k-1, j k} \cdots W_{j 1, j 2} W_{i, j 1}
$$

where $C$ is also the normalizing constant to limit the maximum absolute value of GRSE 
to 1 .

As our aim is to obtain the interaction relationship between the input parameters, the training of ANN will use the same number of input neurons and output neurons, or the same of input and output variables, providing a final matrix that can be comprised by different $m$ and $i$ values within the range of the parameter numbers.

$$
M=\left[\begin{array}{cccc}
G R S E_{11} & G R S E_{12} & \cdots & G R S E_{17} \\
G R S E_{21} & G R S E_{22} & \cdots & G R S E_{27} \\
\cdots & \cdots & \cdots & \cdots \\
G R S E_{71} & G R S E_{72} & \cdots & G R S E_{77}
\end{array}\right]
$$

In the diagonal position stand the general relative strength of each parameter on itself, and the effect of the $m$-th parameter on the $i$-th parameter can be seen in the $m$-th row and $i$-th column of the off-diagonal position.

\subsection{Results and discussion}

\subsubsection{Matrix coding}

As the database contains 254 case histories, the matrix coding process randomly selects 230 groups of data as the training set. The remaining 24 groups are the validation set, which are therefore not employed for training. The network training code is programed in MATLAB to obtain the best network structure measured by the MSE (mean square error) values. The optimal structure of our network is given by two hidden layers with seven neutrons. The coded matrix can be seen in Table 5.2.

The leading diagonal values that represent the coupled effect of one parameter on itself should always be 1.0, and all the leading diagonal values in the GRSE matrix obtained from training work are quite close to 1.0. Therefore, the coded matrix is reliable for use. With the matrix obtained, useful information on the relationship between parameters 
can be obtained. For instance, the excavation span parameter could influence support condition and PPV (note the values of 0.00260 and 0.00199 , respectively). However, the excavation span almost has no effect on the rock mass density (value of -0.00007).

Table 5.2 The coded interaction matrix

\begin{tabular}{lllllllll}
\hline 1 & -0.00198 & -0.00085 & 0.00163 & 0.00009 & 0.00136 & -0.00117 & $\mathbf{0 . 0 0 7 0 9}$ & \\
-0.00050 & 1 & 0.00097 & -0.00064 & -0.00150 & 0.00147 & 0.00077 & $\mathbf{0 . 0 0 5 8 6}$ & \\
0.00028 & 0.00260 & 1 & -0.00037 & -0.00156 & 0.00199 & -0.00007 & $\mathbf{0 . 0 0 6 8 8}$ & \\
0.00026 & -0.00059 & -0.00104 & 1 & -0.00003 & -0.00156 & -0.00137 & $\mathbf{0 . 0 0 4 8 6}$ & \\
0.00069 & 0.00186 & 0.00038 & -0.00075 & 1 & 0.00024 & 0.00084 & $\mathbf{0 . 0 0 4 7 6}$ & \\
-0.00027 & -0.00024 & -0.00110 & 0.00154 & 0.00013 & 1 & -0.00032 & $\mathbf{0 . 0 0 3 6 0}$ & \\
0.00158 & -0.00062 & -0.00069 & -0.00072 & -0.00259 & 0.00242 & 1 & $\mathbf{0 . 0 0 8 6 2}$ & \\
$\mathbf{0 . 0 0 3 6 0}$ & $\mathbf{0 . 0 0 7 9 0}$ & $\mathbf{0 . 0 0 5 0 3}$ & $\mathbf{0 . 0 0 5 6 6}$ & $\mathbf{0 . 0 0 5 9 0}$ & $\mathbf{0 . 0 0 9 0 4}$ & $\mathbf{0 . 0 0 4 5 4}$ & & \\
& & & & & & \\
\hline
\end{tabular}

Notation: P1, $\sigma_{1} / \mathrm{UCS}$; P2, Support condition; P3, Excavation span; P4, Geological factor; P5, Seismic events magnitude; P7, PPV; P9, Rock mass density.

To obtain the weight of each parameter selected using Eq. (5.3), the corresponding C and $\mathrm{E}$ values for each row and column of the matrix are calculated using Eq. (5.1) and Eq. (5.2). The results are also listed in Table 5.2, and Figure 5.9 shows the Cause-Effect relationships for each parameter. The rock mass density parameter (P9) affects the rest of the system most, and the effect of the rest of the system on PPV (P7) is dominant. It can also be noted that the support condition, rock mass density and PPV show larger weights on rock burst damage than other parameters (see the results in Table 5.3).

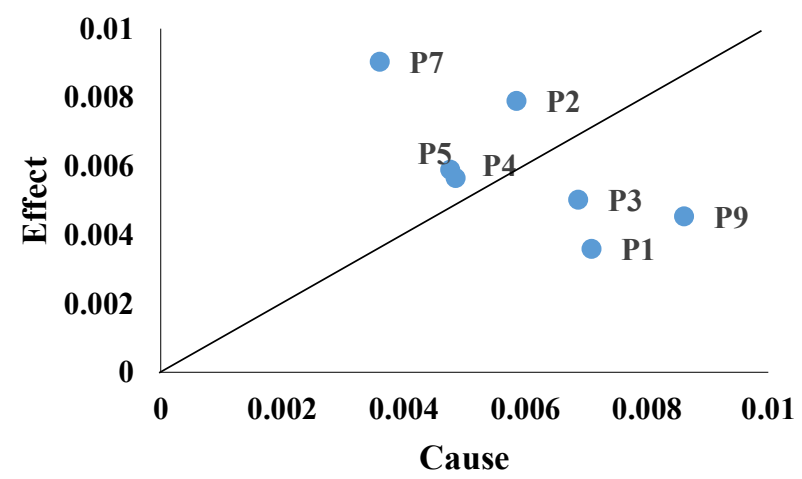

Figure 5.9 The cause and effect for the selected parameters 
Table 5.3 Weight of each parameter for the rock burst damage prediction

\begin{tabular}{ll}
\hline Parameter & Weight \\
\hline$\sigma_{1} /$ UCS (P1) & $\mathbf{1 2 . 8 3}$ \\
Support condition (P2) & $\mathbf{1 6 . 5 0}$ \\
Excavation span (P3) & $\mathbf{1 4 . 2 8}$ \\
Geological factor (P4) & $\mathbf{1 2 . 6 2}$ \\
Seismic event magnitude (P5) & $\mathbf{1 2 . 8 0}$ \\
PPV (P7) & $\mathbf{1 5 . 1 8}$ \\
Rock mass density (P9) & $\mathbf{1 5 . 7 9}$ \\
\hline
\end{tabular}

\subsubsection{Rock burst damage scale index (RDSI)}

As the weights of all the parameters are known, the rock burst damage scale index (RDSI) can be calculated using Eq. (5.4). Figure 5.10 shows the results obtained considering all the case histories of the training database. It can be noted that the four rock burst damage scales have some overlapping zones and, in particular, that the scales of RDSI values corresponding to damage ranges $\mathrm{R} 2$ and $\mathrm{R} 3$ are very similar. This is because - although it is an empirical method considering a wide number of factors-, our proposed RDSI cannot completely differentiate among relatively low levels of rock burst damage when rock burst occurs in an underground excavation. Therefore, for a better implementation of our methods for prediction work, we combine the R2 and R3 damage categories, with the results of the new three scales being shown in Figure 5.11. (Note that results are more distinguishable now.) The average values and standard deviations of RDSI data corresponding to each damage level are listed in Table 5.4. Note that the group corresponding to the R2 and R3 damage categories have almost the same statistics, hence illustrating again the interest of grouping them together. 


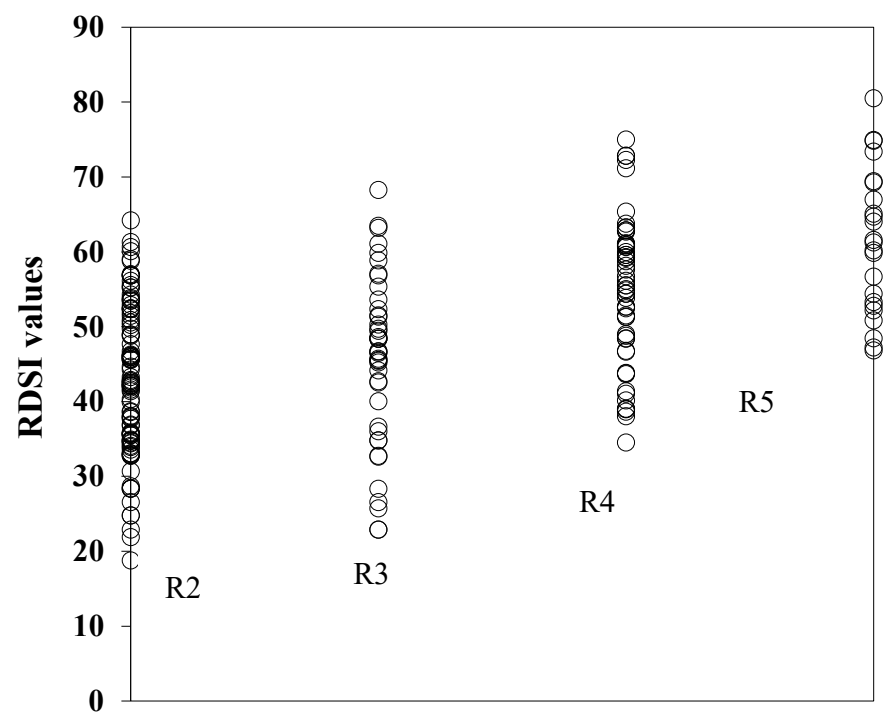

Figure 5.10 Distribution of RDSI (from R2 to R5): values for different levels of rock burst damage, when low different damage scales are considered

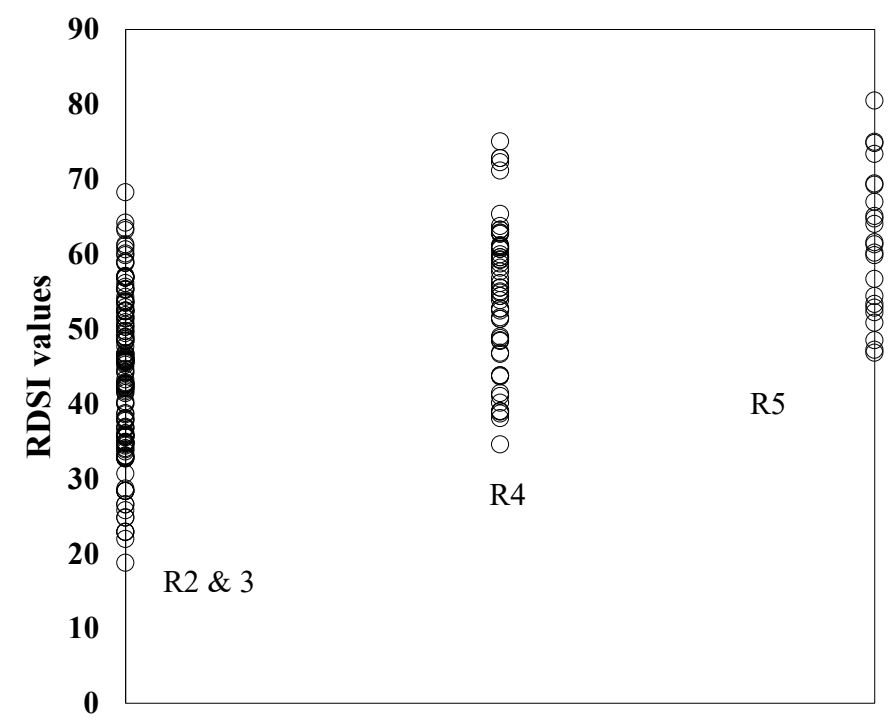

Figure 5.11 Distribution of RDSI (from R2\&3 to R5): values for different levels of rock burst damage, when low different damage scales are not considered and combined

\subsubsection{Probability analyses}

Based on the results above, and given the overlapping that exists among the different damage categories, we can employ a probabilistic approach to better assess the damage categories that most likely associate to a given value of RDSI. To that end, we suppose that the distribution of the RDSI is normal for each damage scale, so that its probability 
density function can be modeled with a normal distribution having the average values and standard deviation values listed in Table 5.4. The results are plotted in Figure 5.12 which, as expected, shows some overlapping areas.

Table 5.4 The statistics of different RDS

\begin{tabular}{lll}
\hline RDS & Average values & Standard deviation \\
\hline R2 & 42.98 & 9.95 \\
R3 & 45.58 & 11.42 \\
R4 & 54.90 & 9.60 \\
R5 & 61.30 & 9.88 \\
R2\&3 & 43.76 & 10.39 \\
\hline
\end{tabular}

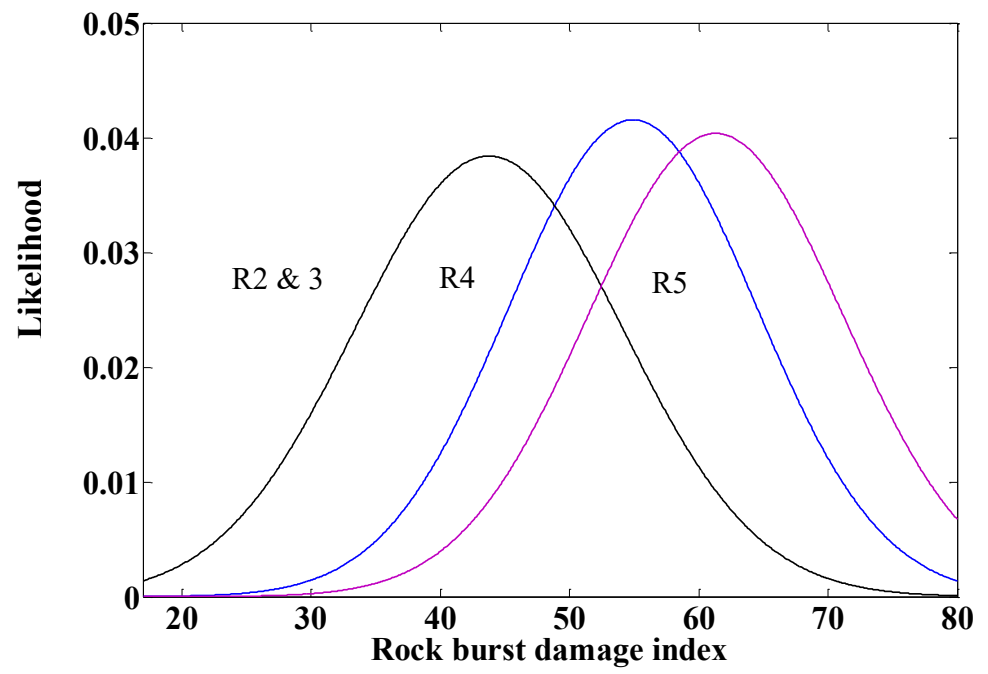

Figure 5.12 PDF of the three damage scales

The likelihood of a certain RDSI value belonging to R2\&3, R4 or R5 (herein denoted to as $\left.y_{1}, y_{2}, y_{3}\right)$ can be estimated using each PDF function separately; that is

$$
\begin{aligned}
& y_{1}=\frac{1}{10.39 \times \sqrt{2 \pi}} e^{-\frac{(R D S I-43.76)^{2}}{2 \times 10.39^{2}}} \\
& y_{2}=\frac{1}{9.60 \times \sqrt{2 \pi}} e^{-\frac{(R D S I-54.90)^{2}}{2 \times 9.60^{2}}} \\
& y_{3}=\frac{1}{9.88 \times \sqrt{2 \pi}} e^{-\frac{(R D S I-61.30)^{2}}{2 \times 9.88^{2}}}
\end{aligned}
$$

For each real case, the values $y_{1}, y_{2}$ and $y_{3}$ can be calculated, and the following formula 
can be employed to obtain the relative likelihood of it belonging to each of the three scales:

$$
\operatorname{Pro}\left(y_{i}\right)=\frac{y_{i}}{\sum_{i}^{3} y_{i}}
$$

With the relative likelihood values of all the training case histories, the likelihood curves of RDSI can be produced, as shown in Figure 5.13.

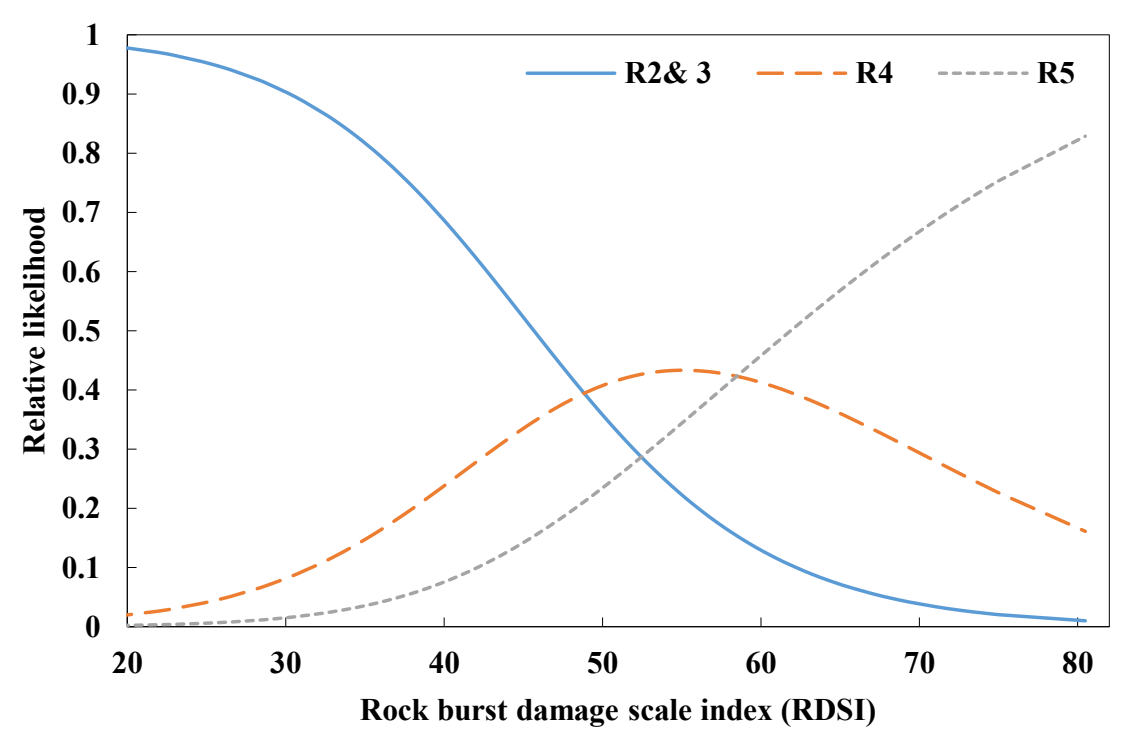

\section{Figure 5.13 Curves of scales likelihood of rock burst damage for a given RDSI}

Therefore, results in Figure 5.13 show that when the RDSI is less than 48.9, the highest likelihood of the rock burst damage corresponds to a damage level of R2\&3, with a R4 damage level being next more likely. For RDSI values between 48.9 and 59.8, the most likely damage scale is R4 although other damage levels are also likely. For RDSI values above 59.8, the most likely damage level is R5.

\subsubsection{RDSI validation}

To further validate our proposed RDSI, 24 new case histories that have not been 
employed in the training process are used for validation. To compare the prediction results, we propose three schemes to estimate damage levels given a RDSI value. The first scheme uses the intersection points of the likelihood curves in Figure 5.13; the second scheme considers the average values of RDSI in each damage scale; and the third scheme employs the minimum RDSI values for each damage scale (34.5 and 46.8 for R4 and R5, respectively). This threshold definition is conservative for the prediction work. Table 5.5 shows the three different thresholds of RDSI to predict the likely damage level of a given new case history. The prediction results computed for the validation database with 24 new case histories that were not employed for training are shown in Table 5.6.

Table 5.5 Three RDSI schemes

\begin{tabular}{llll}
\hline \multirow{2}{*}{ Scheme } & \multicolumn{2}{l}{ RDSI } & \\
\cline { 2 - 4 } & R2\&3 & R4 & R5 \\
\hline 1 & $<48.9$ & $48.9-59.8$ & $>59.8$ \\
2 & $<43.8$ & $43.8-54.9$ & $>54.9$ \\
3 & $<34.5$ & $34.5-46.8$ & $>46.8$ \\
\hline
\end{tabular}

It can be noted that Scheme 1 has the best predictive ability with only 6 wrong cases, among which 4 of them predict a conservative result, hence being safer for prediction on site. And, when the second most likely output is also considered (this is denoted as Scheme $1^{*}$ in Table 5.6), the accuracy rate increases from $75 \%$ to $79.2 \%$. Similarly, results in Table 5.6 show that Schemes 2 and 3 all have a lower accuracy rate although, as expected, Scheme 3 is found to be conservative, which may make it interesting in practical applications. Therefore, our proposed approach can be applied to real case histories on site for rock burst damage prediction. 
Table 5.6 The validation of the test data set

\begin{tabular}{|c|c|c|c|c|c|c|}
\hline \multirow{2}{*}{$\begin{array}{l}\text { Case } \\
\text { history }\end{array}$} & \multirow{2}{*}{$\begin{array}{l}\text { Actual } \\
\text { scale }\end{array}$} & \multirow{2}{*}{ RDSI } & \multicolumn{4}{|c|}{ Predicted scale } \\
\hline & & & Scheme 1 & Scheme 1* & Scheme 2 & Scheme 3 \\
\hline 1 & $\mathrm{R} 4$ & 49.96 & $\mathrm{R} 4$ & & $\mathrm{R} 4$ & $\mathrm{R} 5$ \\
\hline 2 & $\mathrm{R} 2 \& 3$ & 56.03 & R5 & R4 & R5 & R5 \\
\hline 3 & $\mathrm{R} 2 \& 3$ & 45.98 & $\mathrm{R} 2 \& 3$ & & $\mathbf{R 4}$ & $\mathbf{R 4}$ \\
\hline 4 & $\mathrm{R} 5$ & 62.23 & $\mathrm{R} 5$ & & R5 & $\mathrm{R} 5$ \\
\hline 5 & $\mathrm{R} 2 \& 3$ & 31.81 & $\mathrm{R} 2 \& 3$ & & $\mathrm{R} 2 \& 3$ & $\mathrm{R} 2 \& 3$ \\
\hline 6 & $\mathrm{R} 2 \& 3$ & 31.76 & $\mathrm{R} 2 \& 3$ & & $\mathrm{R} 2 \& 3$ & $\mathrm{R} 2 \& 3$ \\
\hline 7 & $\mathrm{R} 4$ & 45.53 & $\mathrm{R} 4$ & & $\mathrm{R} 4$ & $\mathrm{R} 4$ \\
\hline 8 & $\mathrm{R} 2 \& 3$ & 30.68 & $\mathrm{R} 2 \& 3$ & & $\mathrm{R} 2 \& 3$ & $\mathrm{R} 2 \& 3$ \\
\hline 9 & $\mathrm{R} 2 \& 3$ & 33.20 & $\mathrm{R} 2 \& 3$ & & $\mathrm{R} 2 \& 3$ & $\mathrm{R} 2 \& 3$ \\
\hline 10 & R5 & 52.37 & $\mathbf{R 4}$ & R2\&3 & $\mathbf{R 4}$ & $\mathrm{R} 5$ \\
\hline 11 & $\mathrm{R} 4$ & 38.95 & R2\&3 & $\mathrm{R} 4$ & R2\&3 & $\mathrm{R} 4$ \\
\hline 12 & $\mathrm{R} 2 \& 3$ & 42.05 & $\mathrm{R} 2 \& 3$ & & $\mathrm{R} 2 \& 3$ & R4 \\
\hline 13 & $\mathrm{R} 2 \& 3$ & 43.91 & $\mathrm{R} 2 \& 3$ & & $\mathbf{R 4}$ & $\mathbf{R 4}$ \\
\hline 14 & $\mathrm{R} 2 \& 3$ & 64.50 & $\mathrm{R} 5$ & & $\mathrm{R} 5$ & $\mathrm{R} 5$ \\
\hline 15 & $\mathrm{R} 2 \& 3$ & 43.67 & $\mathrm{R} 2 \& 3$ & & $\mathrm{R} 2 \& 3$ & R4 \\
\hline 16 & $\mathrm{R} 2 \& 3$ & 25.92 & $\mathrm{R} 2 \& 3$ & & $\mathrm{R} 2 \& 3$ & $\mathrm{R} 2 \& 3$ \\
\hline 17 & $\mathrm{R} 2 \& 3$ & 54.65 & $\mathbf{R 4}$ & R5 & $\mathbf{R 4}$ & R5 \\
\hline 18 & $\mathrm{R} 2 \& 3$ & 59.18 & $\mathbf{R 4}$ & R5 & R5 & R5 \\
\hline 19 & $\mathrm{R} 2 \& 3$ & 33.26 & $\mathrm{R} 2 \& 3$ & & $\mathrm{R} 2 \& 3$ & $\mathrm{R} 2 \& 3$ \\
\hline 20 & R5 & 62.56 & R5 & & $\mathrm{R} 5$ & $\mathrm{R} 5$ \\
\hline 21 & $\mathrm{R} 2 \& 3$ & 56.20 & R4 & R5 & R5 & R5 \\
\hline 22 & $\mathrm{R} 5$ & 60.65 & $\mathrm{R} 5$ & & $\mathrm{R} 5$ & $\mathrm{R} 5$ \\
\hline 23 & $\mathrm{R} 4$ & 51.29 & $\mathrm{R} 4$ & & $\mathrm{R} 4$ & R5 \\
\hline 24 & $\mathrm{R} 2 \& 3$ & 33.62 & $\mathrm{R} 2 \& 3$ & & $\mathrm{R} 2 \& 3$ & $\mathrm{R} 2 \& 3$ \\
\hline Accuracy & & & $75 \%$ & $79.2 \%$ & $70.8 \%$ & $62.5 \%$ \\
\hline
\end{tabular}

Notation: Scheme $1^{*}$ demostrates the prediction results when the most possible scale is predicted wrong with the curves in Figure 5.13, so this column will show the second possible results instead.

\subsection{Summary}

We propose a new rock burst damage index (RDSI) to predict the scale of damage due to rock burst. Seven parameters that probably affect the rock burst damage are employed - the ratio of total maximum principal stress to UCS (P1), support condition (P2), excavation span (P3), geological factor (P4), seismic event magnitude (P5), approximate peak particle velocity (PPV) (P7) and the rock mass density (P9) — and Rock Engineering Systems (RES) approach is employed to study the interaction 
relationship between the parameters, with Artificial Neural Networks (ANN) using the Backpropagation algorithm being employed to code the interaction matrix.

254 case histories with different scales of damage resulting from 83 recorded seismic events of 13 Australian and Canadian underground rock mines were compiled from the literature to train the $\mathrm{ANN}$, and they were randomly sub-divided into training and validation data sets with 230 and 24 case histories respectively. The global relative strength effects (GRSE) are calculated with the weights resulting from the BP algorithm, and the final interaction matrix can be obtained by the GRSE values between each two parameters. The weights of our employed parameters are then defined and calculated using the trained matrix, and the RDSI values of each case are obtained.

Results show that the interaction matrix obtained by the ANN is reliable: i.e.; the diagonal values are close to 1 and the non-diagonal values show the interactive relation between selected parameters. In addition, the likelihood curves based on the RDSI values of the training case histories show that the three rock burst damage scales considered (R2\&3 combined, R4 and R5) could be predicted probabilistically using specific threshold values $($ RDSI $<48.9$ : R2\&3; $48.9 \leq$ RDSI $<59.8:$ R4; RDSI $\geq 59.8$ : R5).

The proposed RDSI has been validated using 24 case histories. Compared with two other simple threshold values, our proposed threshold has the best predictive ability with only 6 unpredicted cases. Additionally, in actual practice, approaches like Scheme 3 might be also preferable if a conservative prediction is of interest. 


\section{PREDICTING TUNNEL}

SQUEEZING WITH

MACHINE LEARNING

TECHNIQUES

\subsection{Introduction}

As discussed in Chapter 1, several advanced statistical methods have been employed in the literature to address the problem of rock tunnel squeezing. However, the rapid development of this field, as well as the wide variety of tools available, produces the need to investigate which method might be preferable to address this problem.

Therefore, this chapter investigates the performance of several common machine learning tools that have not yet been employed to predict rock tunnel squeezing. The machine learning algorithms selected to be analyzed herein are: Gradient Boosting Machines (GBM), Artificial Neural Networks (ANN), Support Vector Machines 
(SVM), and Multivariate Adaptive Regression Splines (MARS). In addition, the Random Forests Algorithm (RF) and Naïve Bayes (NB) classifier are also included for comparison. For geotechnical and geological engineering, similar analyses have been performed for applications on groundwater level (Mirzavand et al. 2015), dam behavior (Salazar et al. 2015), coal pillar stability (Zhou et al. 2015), slope stability (Suman et al. 2016), and rock burst hazards (Zhou et al. 2016a).

\subsection{Selection of parameters and model construction}

\subsubsection{Selection of parameters}

Based on the literature review of traditional empirical methods to predict squeezing, five main parameters that may influence the occurrence of squeezing are employed in our analyses: Tunnel span or diameter $(B$ or $D)$, Rock tunneling quality index $(Q)$, Tunnel depth $(H)$, Support stiffness $(K)$ and Stress-strength ratio $(S S R)$. Although there are other parameters or indicators that could also affect squeezing — like Rock mass strength $\left(\sigma_{c m}\right)$, Tunnel support pressure $\left(p_{i}\right)$ and pecific weight of the rock mass $(\gamma)$-, they are not employed herein due to the following reasons: (i) the rock mass strength is difficult, time-consuming and expensive to measure in practice (Goel and Singh 2011) and it is therefore often unavailable at early stages of one project; (ii) actual measurements of tunnel support pressure are unavailable until the support is constructed, and their prior estimations are often unfeasible, as suggested by the large discrepancies found between estimations and measurements in real cases (Bhasin and Grimstad 1996); and (iii) the ranges of variability of rock mass unit weights are often limited, so that they can often be assumed constant in this type of practical empirical prediction (Dwivedi et al. 2013).

\subsubsection{Model construction}


Given that up to five parameters are considered herein to predict squeezing, several predictive models could be developed based on different sub-sets being selected among such choice of input parameters. But our objective is to develop models that can achieve a good predictive performance, while still maintaining their simplicity of use. For that reason, only models with up to three input parameters are considered herein. (Note also that models with four or more parameters for their training would need more case histories.)

Table 6.1 summarizes the models considered. Parameters $H$ and $Q$ are considered as input for all models (this is because they are the two most widely employed input parameters for empirical squeezing prediction); so that the additional parameter considered $(D, S S R$ or $K)$ defines whether we are dealing with Model A, B or C (see Table 6.1).

Table 6.1 Input parameters considered with Models A, B and C

\begin{tabular}{llll}
\hline Model & A & B & C \\
\hline H & $\bullet$ & $\bullet$ & $\bullet$ \\
Q & $\bullet$ & $\bullet$ & $\bullet$ \\
D & $\bullet$ & & \\
K & & & $\bullet$ \\
SSR & & $\bullet$ & \\
\hline
\end{tabular}

The number of case histories with information about parameters needed for Model A is 163 (107 squeezing case histories and 56 non-squeezing ones). For Model B, there are 55 squeezing case histories and 37 non-squeezing cases. 77 case histories of squeezing and 28 non-squeezing case histories have enough information to be employed with Model C. The detailed database is presented in Appendix C.

\subsection{Machine learning methods}

\subsubsection{Flowchart of the analyses}


The computations presented herein are conducted with $\mathrm{R}$, an open-source software environment for statistical computing ( $\mathrm{R}$ Core Team 2014). The flowchart of the methodology to test and validate the different machine learning algorithms for rock tunnel squeezing prediction is shown in Figure 6.1.

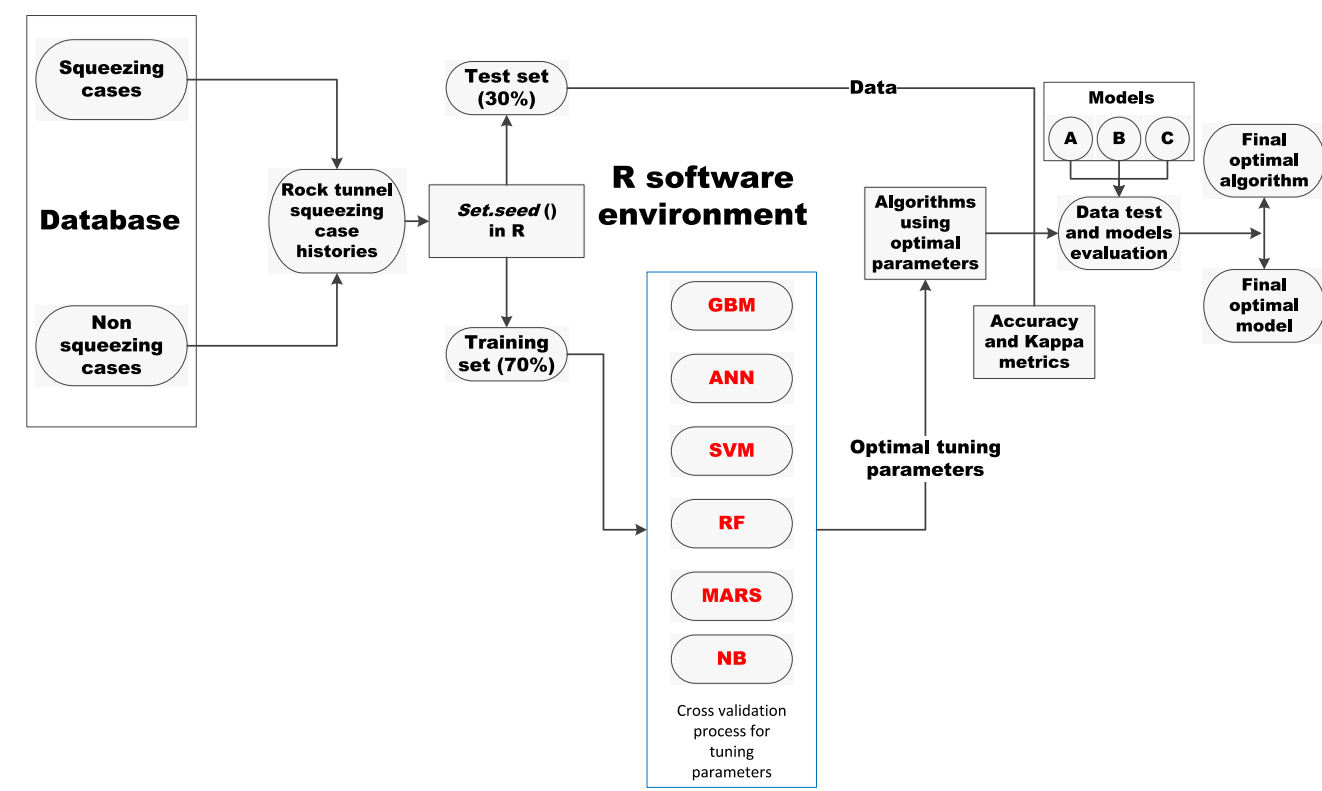

\section{Figure 6.1 Flowchart of the procedure for squeezing prediction in rock tunnels using different machine learning techniques}

As illustrated in Figure 6.1, the database of case histories is divided into two groups: the training set, with $70 \%$ of the available case histories; and the test (or validation) set with the remaining $30 \%$ case histories, which are therefore not employed for training. The division into sub-sets is pseudo-random, as it is conducted with a random selection algorithm, but with a constant (and known) initial seed, that allows the reproducibility of results, and that makes their comparison possible.

The training set is employed to train each proposed model (A, B, or C). Or, in other words, to identify the set of 'fitting parameters' that optimize its performance during 
the training process. Kohavi (1995) suggested a repeated 10-fold cross-validation (CV) for parameter tuning of machine learning algorithms. Therefore, the training set is randomly divided into 10 groups; then, for each group, the algorithm is trained using the other 9 groups, with the originally selected group being used to predict rock squeezing with the trained model. In this chapter, this process is repeated ten times. The results of this exercise, and in particular the average Accuracy values obtained from the cross validation conducted, are then employed to decide the final optimal tuning parameters for the algorithm (see Table 6.2).

For the sake of completeness, the machine learning algorithms employed in this chapter are briefly discussed next, and the related tuning parameters of each parameters are introduced.

\subsubsection{Gradient Boosting Machines (GBM)}

A gradient-descent formulation of boosting methods (GBM) was derived to establish their connection with a statistical framework for regression and classification problems (Freund and Schapire 1997; Friedman et al. 2000; Friedman 2001). The main idea behind this algorithm is to construct new base-learners that are maximally correlated with the negative gradient of the loss function associated with the whole ensemble (Natekin and Knoll 2013).

The algorithm is as follows (Guelman 2012): initialize $f_{0}(x)$ as a constant, $f_{0}(x)=\arg \min _{\rho} \sum_{i=1}^{N} \Psi\left(y_{i}, \rho\right)$, where $\Psi\left(y_{i}, \rho\right)$ is the loss function. Then, for $t$ in 1, $2, \ldots, T$, complete the following procedure:

Step 1: compute the negative gradient

$$
z_{i}=-\left.\frac{\partial}{\partial f\left(x_{i}\right)} \Psi\left(y_{i}, f\left(x_{i}\right)\right)\right|_{f\left(x_{i}\right)=f_{t-1}\left(x_{i}\right)}
$$


Step 2: fit a regression model $g(x)$ for predicting $z_{i}$ form the covariates $x_{i}$

Step 3: choose a gradient descent step size as

$$
\rho=\arg \min _{\rho} \sum_{i=1}^{N} \Psi\left(y_{i}, f_{t-1}\left(x_{i}\right)+\rho g\left(x_{i}\right)\right)
$$

Step 4: update the estimate of $f(x)$ as

$$
f_{t}(x)=f_{t-1}(x)+\rho g(x)
$$

For the GBM, the loss function which represents the losses associated to the errors committed while predicting results using inputs in place of real outputs (Rosasco et al. 2004 ) is selected by the user. In this case, as the response has only 2 values, a Bernoulli function with label " 0 " for non-squeezing condition and " 1 " otherwise is assumed as loss function. Computations are conducted with the $g b m$ R-package (Ridgeway 2004), whose fitting depends on four parameters, related to the total number of iterations (n.trees), the complexity of the trees (interaction.depth), the adaptation rate (shrinkage) and the minimum number of training set samples (n.minnopbsinode). (Note that, for computational efficiency and due to their limited influence on the results, shrinkage and n.minnopbsinode have been set to 0.1 and 10 , respectively.)

\subsubsection{Artificial Neural Networks (ANN)}

A neural network comprises a set of interconnected identical units which can provide mathematical nonlinear models for a wide variety of statistical problems (Haykin 1998). The Backpropagation (BP) algorithm is a common method to train Artificial Neural Networks, and a two phase cycle — propagation and weight updating — are repeated to obtain satisfactory results. (See Chapter 5 for a detailed analysis of the BP algorithm.) 
The R-library nnet (Ripley and Venables 2016) is employed herein for the ANN analyses. It has two mainly influential parameters: the size, which indicates the number of hidden perceptron or units; and the decay (a regularization parameter).

\subsubsection{Support Vector Machines (SVM)}

SVMs are supervised learning models to analyze data for classification and regression (Cortes and Vapnik (1995); see also Smola and Schölkopf (2004) and Moguerza and Muñoz (2006) for a more detailed description). The basic idea is to find the optimal hyperplane separating between two classes, by maximizing the 'margin' between the closest points of the classes. Or, in other words, by maximizing the region bounded by two hyperplanes that separate the two classes.

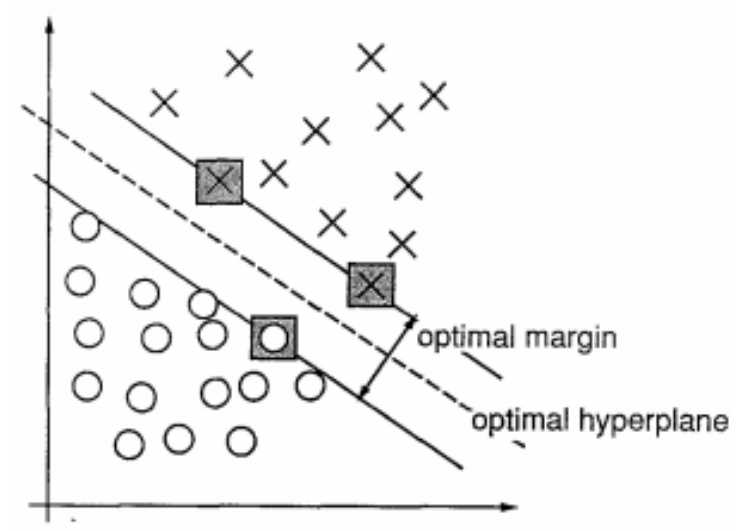

\section{Figure 6.2 An example of a separable problem in a two dimensional space from} Cortes and Vapnik (1995)

Considering a set of collected data, denoted as $\left(x_{i}, y_{i}\right)$ with $i=1,2, \ldots, m$, they can be seperated by a linear separation hyperplane.

$$
w^{T} x+b=0
$$

where $w$ is the weight vector and $b$ is the bias.

For the hyperplane that maximizes the margin, the following relationship can be obtained: 


$$
\left(w^{T} x_{i}+b\right) y_{i} \geq 1, \text { for all } x_{i}, i=1,2, \ldots, m
$$

The distance of the two hyperplanes is $2 /\|w\|$, and the aim is to minimize $\|w\|$ and to prevent data points from falling into the margin. And the training data set that fall on hyperplanes are support vectors.

For the non-linear separation, a hinge loss function is introduced as

$$
\max \left(0,1-y_{i}\left(w^{T} x_{i}+b\right)\right)
$$

and the aim is to minimize the following expression

$$
\left[C \sum_{i=1}^{m} \max \left(0,1-y_{i}\left(w^{T} x_{i}-b\right)\right)\right]+\frac{1}{2}\|w\|^{2}
$$

where $C$ is a constant that controls the relative importance of minimizing the norm of $w$ (which is equivalent to maximizing the margin) and satisfies the margin constraint for each data point. The method of Lagrange multipliers can be employed for the linear solutions. For the non-linear solutions, Gaussian radial basis functions (RBF) are one of the preferable kernel functions, as they have an excellent non-linear forecasting performance (Zhou et al. 2012).

The R-library kernlab with the radial basis function kernel (Karatzoglou et al. 2016) is adopted herein for the SVM analyses. There are two parameters to be tuned: $C$, the cost of constraints violation parameter; and sigma, which indicates the inverse of the kernel.

\subsubsection{Random Forests (RF)}

The RF algorithm was introduced by Breiman (2001). It is based on the use of groups of regression or classification trees in which an additional layer of randomness is added, so that each tree is split using the best among a subset of randomly chosen predictors.

The Random Forests algorithm is as follows (Liaw and Wiener 2002): 
1. Draw $n$ bootstrap samples from the original data set;

2. For each of the bootstrap samples, grow an unpruned classification or regression tree, randomly sampling $m$ of the predictors and choosing the best split from among those variables at each node. Bagging can be thought of as the special case of random forests obtained when $m$ equals the number of predictors;

3. Predict new data by aggregating the predictions of the $n$ trees.

It has two parameters: ntree, or the total number of classification trees; and mtry, or the number of covariates to consider at each split. As the model prediction performance is not very affected by them (Genuer et al. 2010; Kuhn and Johnson 2013), and because the Accuracy is only a little more sensitive to mtry than to ntree (Zhou et al. 2015), we decided to fix the ntree parameter, so that only mtry needs to be tuned.

\subsubsection{Multivariable Adaptive Regression Splines (MARS)}

MARS are a type of non-parametric regression introduced by Friedman (1991). They can be seen as an extension of linear models to simulate nonlinear responses; to that end, they use piecewise linear segments with differing gradients, called splines (Adoko et al. 2013b). Each segment has linear basis functions that are divided by knots, and basis functions are added until the training error decreases above a given threshold. Then, to avoid overfitting, the model is pruned until the best submodel is found. An advantage of MARS is that they are easily interpretable while still being able to work with non-linear problems.

MARS model can be expressed as follows (Friedman 1991):

$$
\hat{f}(x)=\sum_{m=1}^{M} a_{m} B_{m}(x)
$$


where $B_{m}(x)$ is the basis function and $a_{m}$ is a constant coefficient. The basis function has three forms: (i) the constant 1 , (ii) the hinge functions - defined as max $(0, x$-const $)$ or max $(0$, const $-x)$ - and (iii) the combination of hinge functions.

The R-package earth (Milborrow 2011) is employed herein for the MARS analyses. There are two parameters to be tuned: nprune, or the maximum number of terms in the pruned model; and degree, or the maximum degree of interaction.

\subsubsection{Naïve Bayes (NB)}

The naïve Bayes classifier is a simple structure in which the class label is assigned to the parent node by other nodes, having no other connections in the network. The detailed descriptions are presented in Chapter 4, so that they are not repeated here again. The R-package klaR (Roever et al. 2014) is employed herein to work with the NB. There are two parameters to deal with: the first is a logical flag so that, when it is true, a kernel density estimate is used for density estimation; otherwise, a normal density is estimated. The second parameter is $f L$, a factor for Laplace correction.

\subsubsection{Evaluation}

Table 6.2 summarizes the possible tuning values listed for all parameters considered by the different algorithms employed, as well as the optimal parameters that were obtained, for each model, when the training database was employed to fit the models. The Accuracy and Cohen's Kappa values mentioned in Chapter 4 are employed here for the evaluation of performance of machine learning algorithms. 
Table 6.2 Tuning parameters, values and optimal parameters for each model

\begin{tabular}{|c|c|c|c|c|c|}
\hline \multirow{2}{*}{ Method } & \multirow{2}{*}{\multicolumn{2}{|c|}{ Tuning parameters and values }} & \multicolumn{3}{|c|}{$\begin{array}{l}\text { Optimal parameters for } \\
\text { each model }\end{array}$} \\
\hline & & & $\begin{array}{l}\text { Model } \\
\text { A }\end{array}$ & \multirow{2}{*}{$\begin{array}{l}\text { Model } \\
\text { B } \\
250\end{array}$} & \multirow{2}{*}{$\begin{array}{l}\text { Model } \\
\text { C } \\
\end{array}$} \\
\hline \multirow{4}{*}{ GBM } & n.trees & $50,100,150, \ldots, 950,100$ & 850 & & \\
\hline & interaction.depth & $1,2,3,4,5,6,7,8,9$ & 4 & 2 & 1 \\
\hline & shrinkage & 0.1 & 0.1 & 0.1 & 0.1 \\
\hline & n.minopbsinode & 10 & 10 & 10 & 10 \\
\hline \multirow{2}{*}{ ANN } & size & $1,2,3, \ldots, 18,19,20$ & 17 & 18 & 10 \\
\hline & decay & $0,0.001,0.01,0.1,1$ & 0.1 & 0.001 & 0.01 \\
\hline \multirow[t]{2}{*}{ SVM } & $C$ & $\begin{array}{l}0.25,0.5,1,2,4,8,16,32 \\
64,128,256,512\end{array}$ & 128 & 256 & 256 \\
\hline & sigma & $0.0001,0.001,0.01,0.1,1$ & 0.01 & 0.01 & 0.1 \\
\hline \multirow{2}{*}{$\mathrm{RF}$} & ntree & 500 & 500 & 500 & 500 \\
\hline & mtry & $1,2,3,4,5,6$ & 5 & 2 & 4 \\
\hline \multirow[b]{2}{*}{ MARS } & nprune & $1,2,3,4, \ldots, 18,19,20$ & 9 & 8 & 8 \\
\hline & $\begin{array}{l}\text { degree of } \\
\text { interaction }\end{array}$ & $1,2,3,4,5$ & 2 & 2 & 2 \\
\hline \multirow{2}{*}{ NB } & logic flag & True, False & True & True & True \\
\hline & $f L$ & $0,1,2,3,4,5$ & 0 & 0 & 0 \\
\hline
\end{tabular}

\subsection{Results and discussion}

\subsubsection{Predictive accuracy analyses}

To allow a more reliable comparison of models, the training and test sets employed for training and validation are identical for all algorithms considered. All the models (A, B and C) are trained using the six machine learning algorithms discussed above, with the 'optimal' parameters that correspond to each model, algorithm and data set; and with their corresponding performances being measured using the Accuracy and Kappa values. Results are listed in Table 6.3.

Figure 6.3 summarizes the performance results —as measured by the corresponding Accuracy and Kappa values - of the training process, considering all combinations of models and algorithms. 
Table 6.3 Results of the training performance with different machine learning methods for the three models considered

\begin{tabular}{lllllll}
\hline Model & A & & B & & C \\
\hline Method & Accuracy & Kappa & Accuracy & Kappa & Accuracy & Kappa \\
\hline GBM & $\mathbf{9 0 . 8 \%}$ & $\mathbf{0 . 7 8 4}$ & $\mathbf{8 1 . 7 \%}$ & $\mathbf{0 . 6 1 1}$ & $93.8 \%$ & 0.782 \\
ANN & $\mathbf{8 8 . 7 \%}$ & $\mathbf{0 . 7 2 0}$ & $73.3 \%$ & 0.442 & $\mathbf{9 5 . 7 \%}$ & $\mathbf{0 . 8 4 4}$ \\
SVM & $83.1 \%$ & 0.558 & $71.3 \%$ & 0.375 & $\mathbf{9 7 . 8 \%}$ & $\mathbf{0 . 9 1 1}$ \\
RF & $\mathbf{8 7 . 0 \%}$ & $\mathbf{0 . 6 9 3}$ & $\mathbf{8 7 . 3 \%}$ & $\mathbf{0 . 7 3 6}$ & $\mathbf{9 7 . 0 \%}$ & $\mathbf{0 . 8 9 6}$ \\
MARS & $86.8 \%$ & 0.680 & $70.6 \%$ & 0.399 & $94.4 \%$ & 0.807 \\
NB & $79.2 \%$ & 0.449 & $\mathbf{7 8 . 4 \%}$ & $\mathbf{0 . 5 4 6}$ & $90.1 \%$ & 0.661 \\
\hline
\end{tabular}

After the 'optimal' parameters have been tuned with the training data, the validation set with the remaining 30 percent of case histories is employed to test the performance of each algorithm with such 'optimal' parameters. The resulting confusion matrices are listed in Table 6.4 and the computed Accuracy and Kappa values are listed in Table 6.5.
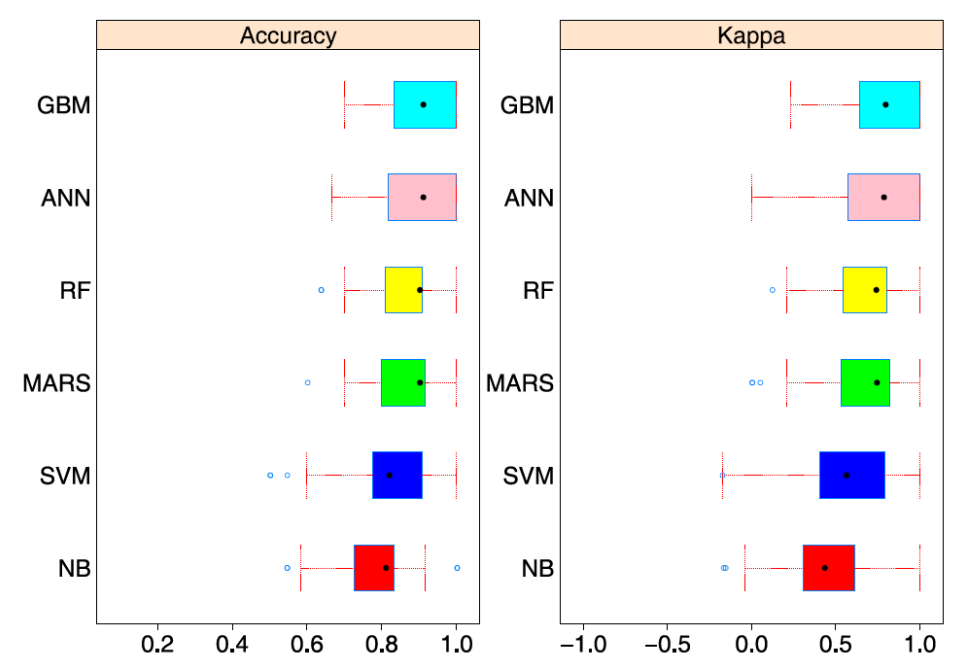

(a) Model A 

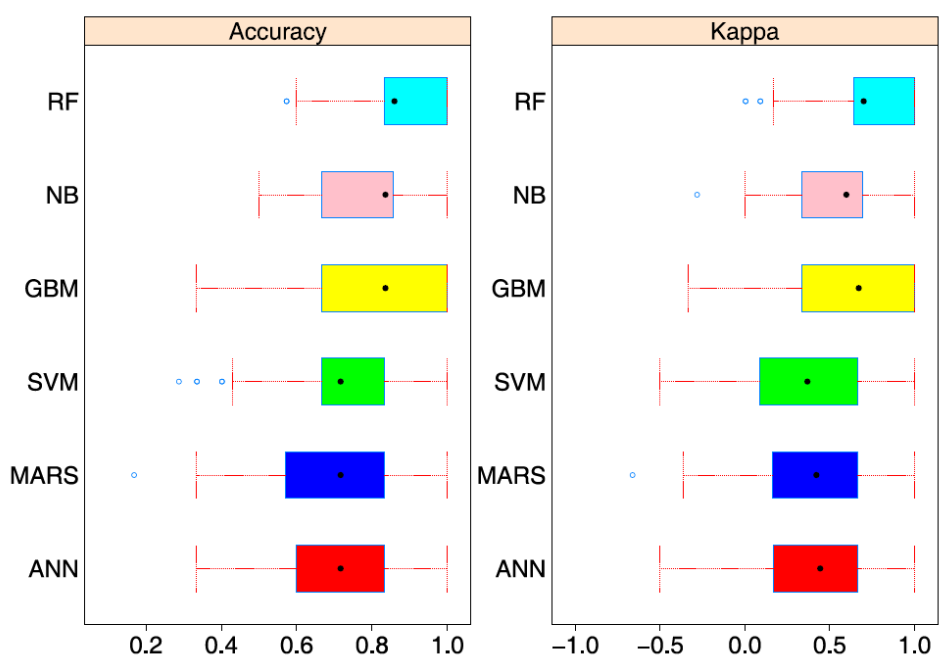

(b) Model B

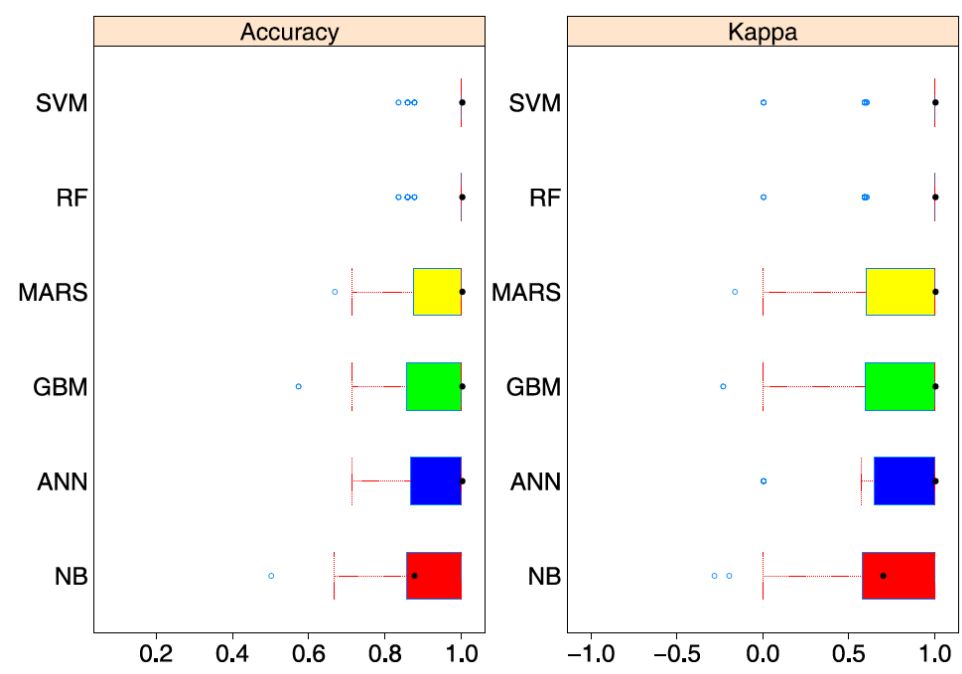

(c) Model C

Figure 6.3 Boxplot distribution of results obtained during the train process (Accuracy and Kappa metrics with different machine learning techniques)

Results show that the Accuracy ranges between (approximately) 73\% and 95\% for the different algorithms, models and databases. Similarly, the Cohen's Kappa results also suggest that predictions computed with the different algorithms and models are adequate, as they are always higher than 0.4 , which is the value proposed by Landis and Koch (1977) as a threshold indication of good agreement. 
Table 6.4 Confusion matrices obtained during validation

\begin{tabular}{|c|c|c|c|c|c|c|c|c|}
\hline \multirow[t]{2}{*}{ Algorithm } & \multirow[b]{2}{*}{ Actual } & \multicolumn{2}{|c|}{ Model A } & \multicolumn{2}{|c|}{ Model B } & \multicolumn{2}{|c|}{ Model C } & \multirow[t]{2}{*}{ Predicted } \\
\hline & & No & Yes & No & Yes & No & Yes & \\
\hline \multirow[t]{2}{*}{ GBM } & & 18 & 3 & 7 & 3 & 10 & 1 & No \\
\hline & & 3 & 30 & 3 & 17 & 2 & 22 & Yes \\
\hline \multirow[t]{2}{*}{ ANN } & & 15 & 2 & 9 & 3 & 8 & 0 & No \\
\hline & & 6 & 31 & 1 & 17 & 4 & 23 & Yes \\
\hline \multirow[t]{2}{*}{ SVM } & & 15 & 6 & 6 & 0 & 7 & 0 & No \\
\hline & & 6 & 27 & 4 & 20 & 5 & 23 & Yes \\
\hline \multirow[t]{2}{*}{ RF } & & 18 & 4 & 10 & 3 & 10 & 0 & No \\
\hline & & 3 & 29 & 0 & 17 & 2 & 23 & Yes \\
\hline \multirow[t]{2}{*}{ MARS } & & 19 & 4 & 9 & 1 & 7 & 0 & No \\
\hline & & 2 & 29 & 1 & 19 & 5 & 23 & Yes \\
\hline \multirow[t]{2}{*}{ NB } & & 9 & 2 & 5 & 3 & 6 & 0 & No \\
\hline & & 12 & 31 & 5 & 17 & 6 & 23 & Yes \\
\hline
\end{tabular}

If the best algorithms for each model are considered, results show that the GBM and MARS algorithms provide the best predictive results for Model A, with the RF algorithm providing similarly adequate results. For Model B, MARS and RF provide the best predictions, with ANN providing similarly good (although slightly worse) results. Finally, the best results for Model $\mathrm{C}$ are obtained with the RF and GBM algorithms, with ANN providing similarly good (although slightly worse) results. Results also show that Model C provides prediction results that are, in general and for most algorithms, better than prediction results computed with Models A and B.

To be able to compare with the predictions of other squeezing prediction methods that are commonly employed in the literature, Table 6.5 also presents results of predictions conducted with Singh et al. (1992) and Jimenez and Recio (2011) approaches, which can be summarized respectively, by Eq. (6.9) and (6.10).

$$
\begin{gathered}
H \geq 350 Q^{1 / 3} \\
H \geq 424.4 Q^{0.32}
\end{gathered}
$$


Table 6.5 Results of the validation performance with different machine learning methods for the three models considered

\begin{tabular}{lllllll}
\hline Model & A & & B & & C \\
\hline Method & Accuracy & Kappa & Accuracy & Kappa & Accuracy & Kappa \\
\hline GBM & $\mathbf{8 8 . 9 \%}$ & $\mathbf{0 . 7 6 6}$ & $80.0 \%$ & 0.550 & $\mathbf{9 1 . 4 \%}$ & $\mathbf{0 . 8 0 1}$ \\
ANN & $85.2 \%$ & 0.677 & $\mathbf{8 6 . 7 \%}$ & $\mathbf{0 . 7 1 4}$ & $\mathbf{8 8 . 6 \%}$ & $\mathbf{0 . 7 2 4}$ \\
SVM & $77.8 \%$ & 0.533 & $86.7 \%$ & 0.667 & $85.7 \%$ & 0.648 \\
RF & $\mathbf{8 7 . 0 \%}$ & $\mathbf{0 . 7 3 0}$ & $\mathbf{9 0 . 0 \%}$ & $\mathbf{0 . 7 9 1}$ & $\mathbf{9 4 . 3 \%}$ & $\mathbf{0 . 8 6 8}$ \\
MARS & $\mathbf{8 8 . 9 \%}$ & $\mathbf{0 . 7 7 0}$ & $\mathbf{9 3 . 3 \%}$ & $\mathbf{0 . 8 5 0}$ & $85.7 \%$ & 0.648 \\
NB & $74.1 \%$ & 0.403 & $73.3 \%$ & 0.368 & $82.9 \%$ & 0.568 \\
Singh et al. (1992)* & $81.5 \%$ & 0.589 & $76.7 \%$ & 0.462 & $85.7 \%$ & 0.648 \\
Jimenez and Recio & $85.2 \%$ & 0.683 & $73.3 \%$ & 0.429 & $88.6 \%$ & 0.736 \\
$(2011)^{*}$ & & & & & & \\
\hline
\end{tabular}

* Note that these models predict using $\mathrm{H}$ and Q only. Therefore, results presented in this Table correspond to their use with the same sub-data sets used for validation and testing of Models A, B and $\mathrm{C}$, and not with the predictions of Models A, B and C.

The reader should note, however, that they correspond to the use of such methods with the sub-sets of the database that were employed to assess the performance of the different algorithms with Models A, B and C; and, perhaps more importantly, the reader should also note that they predict using only two input parameters (H and Q) and not three as Models A, B and C do.

Results indicate that their performance is similar — with Jimenez and Recio (2011)'s method outperforming Singh et al. (1992)'s method with the sub-sets of validation case histories developed for Model A and C, and with the contrary situation with sub-set Band that they often provide comparable results to the other methods (which, as indicated above, use three input parameters instead of two).

\subsubsection{Stability analyses}

The performance of the six algorithms conducted have also been indicated in Figure 6.4 and Table 6.6 for an intuitive comparison. In Figure 6.4, a three-dimensional coordinate system is established with three axes that represent the three models proposed in this chapter. The value in each axis reflects the relative performance of 
each algorithm with the corresponding model. To that end, in Table 6.6, we assign a three-dimensional coordinate to each algorithm's performance according to its training and validation results with each model. Table 6.6 also lists the accuracy performance of each algorithm (ranked by the distance between the coordinate that indicate the validation performance and the origin $(0,0,0))$ and their stability performance (ranked by the distance between training and validation performances). For improved clarity, Figure 6.5 represents the information presented in Figure 6.4 using two-dimensional coordinate systems.

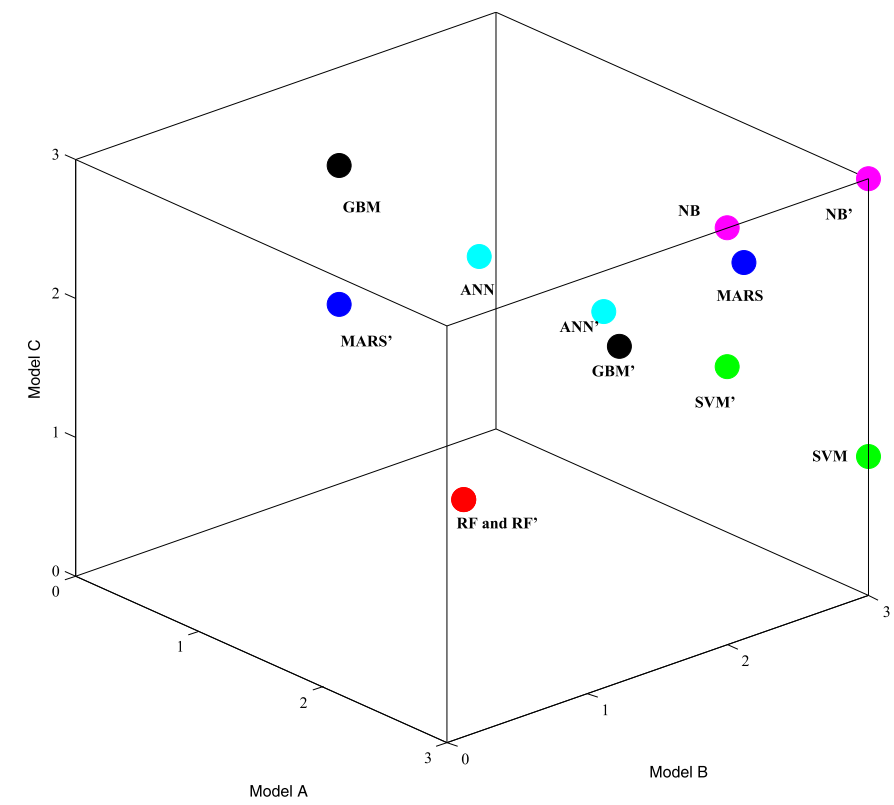

Figure 6.4 3D distribution of relative performances, with different algorithms, during training and validation (For instance, the point named GBM represents the training set performance and GBM' represents the performance with the validation data set) 


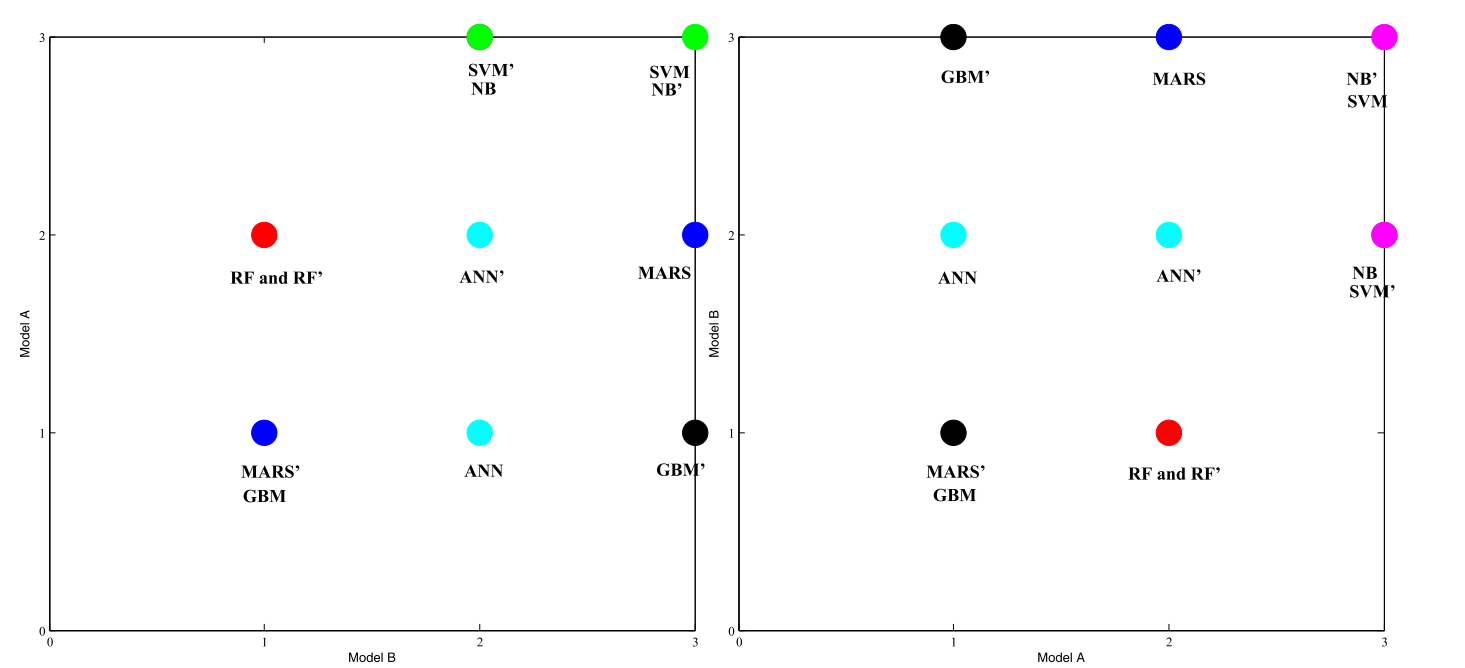

(a) Model A

(b) Model B

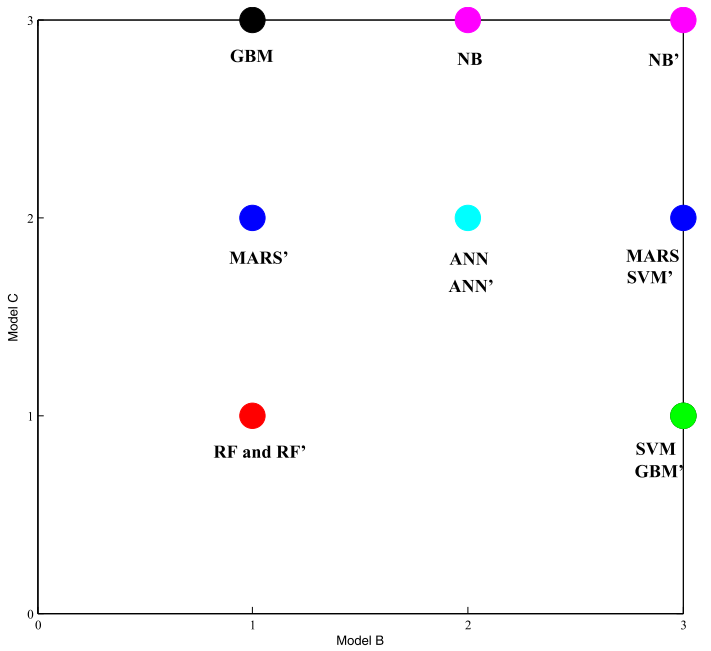

(c) Model C

Figure 6.5 2D distribution of relative performances with different algorithms

Combined with the boxplots in Figure 6.3, it can be seen that the RF method has the shortest interquartile range (IQR) among all the three models, so that it can be recognized as a more stable algorithm to be trained with repeated CV tests. And its performance coordinates $((2,1,1)$ during both training and validation) also reflect its stability during both stages. 
However, other algorithms (see, for instance, the performance of MARS and GBM in

Figure 6.3 and 6.4) show a larger training IQR and a reduced stability during validation.

In other words, although some algorithms may have better accuracy performance with some models, their lower stability make them to have more unstable predictive results.

Table 6.6 Performance coordinates and ranks of different machine learning techniques for tunnel squeezing prediction

\begin{tabular}{|c|c|c|c|c|}
\hline Methods & $\begin{array}{l}\text { Training set } \\
\text { performance } \\
\text { coordinates } \\
\text { (Models A, B } \\
\text { and C) }\end{array}$ & $\begin{array}{l}\text { Validation set } \\
\text { performance } \\
\text { coordinates } \\
\text { (Models A, B } \\
\text { and C) }\end{array}$ & $\begin{array}{l}\text { Accuracy } \\
\text { performance } \\
\text { rank }\end{array}$ & $\begin{array}{l}\text { Stability } \\
\text { performance } \\
\text { rank }\end{array}$ \\
\hline GBM & $(1,1,3)$ & $(1,3,1)$ & 3 & 6 \\
\hline ANN & $(1,2,2)$ & $(2,2,2)$ & 4 & 2 \\
\hline SVM & $(3,3,1)$ & $(3,2,2)$ & 5 & 4 \\
\hline $\mathrm{RF}$ & $(2,1,1)$ & $(2,1,1)$ & 1 & 1 \\
\hline MARS & $(2,3,2)$ & $(1,1,2)$ & 1 & 5 \\
\hline NB & $(3,2,3)$ & $(3,3,3)$ & 6 & 2 \\
\hline
\end{tabular}

Notation: For the $(\mathrm{x}, \mathrm{y}, \mathrm{z})$ coordinates provided, " 1 " means the first two highest Accuracy values in Tables 6.3 and 6.5, "2" means the middle two values, and " 3 " means the last two values of Accuracy. When the Accuracy value is the same, the Cohen' Kappa value will be compared to assign the number.

Similarly, the results show that Model $\mathrm{C}$ has the best predictive performance, and a much better stability than Models A and B. And, since the RF algorithm has been shown to provide the best accuracy and stability performance (during both training and validation) for the three models considered, it could be argued that the joint use of Model $\mathrm{C}$ and the RF algorithm provides the optimal combination of tools (of those considered herein) for prediction of squeezing in rock tunnels.

\subsubsection{Sensitivity analyses}

As the three models considered use different parameters for prediction, the importance of these parameters (or the sensitivity of results to changes in them) is also studied. The command varImp in R-package caret is employed to analyze the sensitivity of different 
parameters (Kuhn and Johnson 2013) to predictions obtained with the RF algorithm, as such algorithm has provided the best performance among the three models considered.

Results are listed in Table 6.7. They show that, for Models A and $\mathrm{B}, Q$ is the most influential parameter, with $H$ being the second, and with the other two parameters $(D$ or $S S R$ ) having the least importance. However, in Model C, the support stiffness $(K)$ has the largest importance, with $H$ and $Q$ being next. As Model $\mathrm{C}$ has a higher predictive performance than the other two models, it could be argued that it is the most important parameter for squeezing prediction, with $H$ and $Q$ being also relatively important. (A similar conclusion has been reported by Feng and Jimenez (2015).) It is therefore considered that the support stiffness should be an important supplementary parameter to be included in improved future models to predict squeezing in rock tunnels.

Table 6.7 Results of the parameter importance or sensitivity analyses using the algorithm with three predictive models

\begin{tabular}{llll}
\hline Parameters & Model A & Model B & Model C \\
\hline $\mathrm{H}$ & 26.95 & 14.88 & 25.77 \\
$\mathrm{Q}$ & $\mathbf{4 1 . 4 5}$ & $\mathbf{3 2 . 7 1}$ & 13.84 \\
$\mathrm{D}$ & 12.16 & $*$ & $*$ \\
$\mathrm{SSR}$ & $*$ & 8.64 & $*$ \\
$\mathrm{~K}$ & $*$ & $*$ & $\mathbf{2 7 . 0 3}$ \\
\hline
\end{tabular}

\subsection{Summary}

We explore the performance of six machine learning algorithms - GBM, ANN, SVM, MARS, RF and NB - when they are employed to predict rock tunnel squeezing using different predictive models. Such models are constructed using different three-out-offive combinations of input parameters that are found to be relevant for tunnel squeezing, so that all models include the Rock tunneling quality index $(Q)$ and Tunnel depth $(H)$ as input, with an additional parameter being employed to consider the influence of Tunnel span or diameter ( $B$ or $D$; Model A); of Stress-strength ratio (SSR; Model B); 
or of Support stiffness ( $K$; Model $\mathrm{C})$. To assure an effective implementation and a reduced computational time, the R-environment for statistical computing is employed as a toolbox within which the parameters of each algorithm can be easily tuned while evaluating its performance.

A database with 166 case histories of squeezing and non-squeezing cases was compiled from the literature, and it was randomly sub-divided into training and validation data sets that were kept constant during the subsequent analyses. Optimal tuning parameters for each algorithm are obtained using repeated 10 -fold cross validation exercises with the training data, and such fitted models are then further validated by analyzing their performance — in terms of predictive accuracy and stability — with the validation data set.

Results show that the Random Forests (RF) algorithm provides the best performance for the three models considered. Other methods — such as MARS and GBM- also have a good average performance, although they are found to be not so stable. Similarly, results suggest that Model $\mathrm{C}$ has a better predictive performance than Model A or B. It is therefore suggested that Model $\mathrm{C}$ can be combined with the RF algorithm for future analyses of rock tunnel squeezing. And the results of the sensitivity analyses conducted suggest that the tunnel support stiffness $(K)$ could be the most influential parameter for prediction of tunnel squeezing, and confirm that $H$ and $Q$ are also important. 


\section{CONCLUSIONS AND FUTURE WORK}

\subsection{Conclusions}

This dissertation develops and presents novel approaches based on machine learning techniques to predict underground tunnel hazards associated to rock burst and to tunnel squeezing. Three different databases collected from different types of underground projects from all over the world are employed for the training and validation work, and the parameters that could mainly affect the rock burst and rock tunnel squeezing are employed to establish simple classifiers and models. Four main problems in previous empirical and machine learning methods for hazard prediction have been addressed, with results showing that the type of advanced statistical and machine learning methods employed herein are a viable tool to improve our prediction about the likely occurrence of rock burst and squeezing.

In particular, this dissertation provides an advancement of the state-of-the-art in relation to the following problems associated to hazard prediction in underground works: (i) we 
develop two new approaches (a linear classifier and a Bayesian Network) to probabilistically predict the likely occurrence of rock burst in the long term ( $\mathrm{Li}$ et al. 2017a; Li and Jimenez 2017; Li et al. 2017b). As previously available methods, our proposed methods use only input data which are relatively easy to obtain at the early stages of one project, but they have the advantage with respect to previous approaches that they can be used in the context of risk analyses (because they provide a probabilistic estimation of the possible outcomes) and, in the case of Bayesian networks, that they can be employed even in the presence of incomplete data; (ii) as typical machine learning algorithms are often beyond the capabilities of many engineers on site due to complex procedures for model establishment, training and validation, we develop a simple rock burst damage scale index (or RDSI) to discriminate the short-term rock burst damage scale (combined with the proposed simple equations for long-term rock burst prediction derived with the linear classifier, they are two easily applicable tools can be employed to estimate rock burst occurrence on site); and (iii) we trained, compared and validated six different machine learning algorithms, to better understand the predictive accuracy and stability of different methods in the context of these problems, and to be able to propose an optimal combination for joint use of model and algorithm for rock tunnel squeezing prediction. In other words, this dissertation provides two new and simple ways to apply advanced machine learning techniques as follows: simple and easy applicable equations or indices that were trained from machine learning methods; and simple structure models that could predict results by running simple codes.

A cautionary note should be added to remind readers that the methods proposed in the dissertation are only empirical solutions to complex underground problems, so that, 
although they are considered as useful tools to preliminarily anticipate and evaluate underground tunnel hazards, and to guide subsequent control and prevention measures, they should not be substitutes for other methods such as numerical simulation, field testing, and monitoring, among others, that are commonly employed in current practice to deal with those hazards. Similarly, as more case histories become available in the future, the three databases developed for this thesis should be extended, so that the predictive models can be updated to further improve future predictions. In addition, the collected databases have limits that affect the limits of applicability of the proposed models.

\subsection{Suggestion for future work}

Future work can be conducted in the following areas:

(1) For the short-term rock burst prediction, more data sets from sites in real underground excavations are needed so that they can be employed for better prediction work. Aspects such as micro seismic and acoustic emission events, as well as data on electromagnetic emissions, stress conditions, etc. can be collected and analyzed using methods employed herein, and their future improvements. For instance, the Dynamic Bayesian Networks $(\mathrm{DBN})$ - a special type of BNs that relate variables to each other over adjacent time steps, based on the general BN framework - could be employed for the real time prediction. As BNs could also update the network as new data are acquired, it is likely that a real-time prediction system can be established, so that the systems for data acquisition on site are connected to the computational tools employed to predict the rock burst risk synchronously.

(2) The methodological approaches developed for this dissertation can also be employed to assess hazards associated to other problems in underground construction. 
For instance, for underground pillar failure prediction (Recio-Gordo and Jimenez 2012; Recio Gordo 2014), an improved linear classifier could be developed. To that end, two different pillar stability databases from rock and coal mines could be used so that, building on the experience obtained from the long-term rock burst prediction in this thesis, the tree model can be combined with linear logistic regression to discriminate the pillar stability. The resulting intuitive tree structure and the linear model could also employed for risk analyses.

(3) Machine learning techniques have also a great potentials to solve other geotechnical problems. However, the predictive and stability performance of each algorithm are different and can be affected by the database collected. A general integrated system (or algorithm) including several machine learning methods can be established to predict the rock burst or squeezing hazards with different input models, and the optimal model and algorithm can be automatically selected to be employed for the final prediction work.

(4) Though machine learning techniques have been shown to be able to predict the underground hazards effectively, numerical simulations are also an effective tool to assess the real influence of the more relevant parameters that have obtained in the sensitivity analyses of machine learning methods, and to optimize the simulation results. For example, numerical packages such as PFC (Itasca 2014) and FLAC (Itasca 2012) software, among others, can be coupled to better analyze rock burst occurrence. The damage level under different support, excavation, and geological conditions could then be simulated and, once the failure process is monitored and compared, results of the numerical simulations can be compared with the measurements. 


\section{REFERENCES}

Abadi, M., Barham, P., Chen, J., et al., TensorFlow: a system for large-scale machine learning. In: Proceedings of the 12th USENIX conference on Operating Systems Design and Implementation, Savannah, GA, USA, USENIX Association, 3026899, pp 265-283.

Adoko, A.C., Gokceoglu, C., Wu, L., et al., 2013a. Knowledge-based and data-driven fuzzy modeling for rockburst prediction. Int J Rock Mech Min. 61, 86-95.

Adoko, A.C., Jiao, Y.Y., Wu, L., et al., 2013b. Predicting tunnel convergence using Multivariate Adaptive Regression Spline and Artificial Neural Network. Tunn Undergr Sp Tech. 38, 368-376.

Aguilera, P., Fernández, A., Fernández, R., et al., 2011. Bayesian networks in environmental modelling. Environ Modell Softw. 26, 1376-1388.

Akaike, H., 1974. A new look at the statistical model identification. Ieee T Automat Contr. 19, 716-723.

Alcott, J.M., Kaiser, P.K., Simser, B.P., 1998. Use of microseismic source parameters for rockburst hazard assessment. Pure Appl Geophys. 153, 41-65.

Aliha, M.R.M., Ayatollahi, M.R., 2012. Analysis of fracture initiation angle in some cracked ceramics using the generalized maximum tangential stress criterion. Int J Solids Struct. 49, 1877-1883.

Bai, Y., Deng, J., Dong, L., et al., 2009. Fisher discriminant analysis model of rock burst prediction and its application in deep hard rock engineering. Journal of Central South University (Science and Technology). 40, 1417-1422.

Barla, G., 1995. Squeezing rocks in tunnels. ISRM News Journal. 2, 44-49.

Barla, G., Pelizza, S., 2000. TBM tunnelling in difficult ground conditions. In: ISRM International Symposium, Melbourne, Australia. 
Bayes, M., Price, M., 1763. An essay towards solving a problem in the doctrine of chances. By the Late Rev. Mr. Bayes, F. R. S. Communicated by Mr. Price, in a Letter to John Canton, A. M. F. R. S. Philosophical Transactions. 53, 370-418.

Bhasin, R., Grimstad, E., 1996. The use of stress-strength relationships in the assessment of tunnel stability. Tunn Undergr Sp Tech. 11, 93-98.

Borg, A., Bjelland, H., Njå, O., 2014. Reflections on Bayesian Network models for road tunnel safety design: A case study from Norway. Tunn Undergr Sp Tech. 43, 300-314.

Brauner, G., 1994. Rockbursts in coal mines and their prevention. AA Balkema, Avereest, Netherlands.

Breiman, L., 2001. Random forests. Mach Learn. 45, 5.

Cai, M., 2013. Principles of rock support in burst-prone ground. Tunn Undergr Sp Tech. $36,46-56$.

Cai, W., Dou, L., Li, Z., et al., 2016a. Verification of passive seismic velocity tomography in rock burst hazard assessment. Chinese Journal Geophysics. 59, 252-262.

Cai, W., Dou, L., Si, G., et al., 2016b. A principal component analysis/fuzzy comprehensive evaluation model for coal burst liability assessment. Int J Rock Mech Min. 81, 62-69.

Cao, A., Dou, L., Cai, W., et al., 2015. Case study of seismic hazard assessment in underground coal mining using passive tomography. Int J Rock Mech Min. 78, 1-9.

Cao, A., Dou, L., Wang, C., et al., 2016. Microseismic precursory characteristics of rock burst hazard in mining areas near a large residual coal pillar: a case study from Xuzhuang coal mine, Xuzhou, China. Rock Mech Rock Eng. 49, 4407-4422.

Cheng, J., Greiner, R., Comparing Bayesian network classifiers. In: Laskey, K.,Prade, H. (eds) Proceedings of the Fifteenth conference on Uncertainty in artificial intelligence, Stockholm, Sweden, Morgan Kaufmann Publishers Inc., San Francisco, USA, pp 101108.

Chow, C., Liu, C., 1968. Approximating discrete probability distributions with dependence trees. Ieee T Inform Theory. 14, 462-467.

Cohen, J., 1960. A Coefficient of agreement for nominal scales. Educ Psychol Meas. 20, 37-46.

Cook, N.G.W., 1966. Rock mechanics applied to the study of rockbursts. South African Institute of Mining and Metallurgy, South Africa.

Cooper, G.F., Herskovits, E., 1992. A Bayesian method for the induction of probabilistic networks from data. Mach Learn. 9, 309-347.

Cortes, C., Vapnik, V., 1995. Support-vector networks. Mach Learn. 20, 273-297.

Curtin, R.R., Cline, J.R., Slagle, N.P., et al., 2013. MLPACK: A scalable C++ machine 
learning library. J Mach Learn Res. 14, 801-805.

Dalgıç, S., 2002. Tunneling in squeezing rock, the Bolu tunnel, Anatolian Motorway, Turkey. Eng Geol. 67, 73-96.

Dancy, C., Reidy, J., 2004. Statistics without maths for psychology. Pearson Education Limited, New York.

Debernardi, D., Barla, G., 2009. New viscoplastic model for design analysis of tunnels in squeezing conditions. Rock Mech Rock Eng. 42, 259-288.

Ding, X., Wu, J., Li, J., 2003. Artificial neural network for forecasting and classification of rockbursts. Journal of Hohai University (Natural Science). 31, 424-427.

Dong, L., Li, X., Ming, X., et al., 2011. Comparisons of Random Forest and Support Vector Machine for Predicting Blasting Vibration Characteristic Parameters. Procedia Engineering. 26, 1772-1781.

Dou, L., Chen, T., Gong, S., et al., 2012. Rockburst hazard determination by using computed tomography technology in deep workface. Safety Sci. 50, 736-740.

Dou, L., Lu, C., Mu, Z., et al., 2009. Prevention and forecasting of rock burst hazards in coal mines. Mining Science and Technology (China). 19, 585-591.

Dou, L., Zhao, C., Yang, S., et al., 2006. Prevention and control of rock burst in coal mine. China University of Mining and Technology Press, Xuzhou, China.

Dreiseitl, S., Ohno-Machado, L., 2002. Logistic regression and artificial neural network classification models: a methodology review. J Biomed Inform. 35, 352-359.

Dwivedi, R., Singh, M., Viladkar, M., et al., 2013. Prediction of tunnel deformation in squeezing grounds. Eng Geol. 161, 55-64.

Fawcett, T., 2006. An introduction to ROC analysis. Pattern Recogn. Lett. 27, 861-874.

Feng, X., Jimenez, R., 2015. Predicting tunnel squeezing with incomplete data using Bayesian networks. Eng Geol. 195, 214-224.

Feng, X., Wang, L., 1994. Rockburst prediction based on neural networks. T Nonferr Metal Soc. 4, 7-14.

Freund, Y., Schapire, R.E., 1997. A decision-theoretic generalization of on-line learning and an application to boosting. J Comput Syst Sci. 55, 119-139.

Friedman, 2001. Greedy function approximation: A gradient boosting machine. Ann Stat. 29, 1189.

Friedman, J.H., 1991. Multivariate Adaptive Regression Splines. Ann Stat. 19, 1-67.

Friedman, J.H., Hastie, T., Tibshirani, R., 2000. Additive logistic regression: a statistical view of boosting (with discussion and a rejoinder by the authors). Ann Stat. $28,337-407$. 
Friedman, N., Geiger, D., Goldszmidt, M., 1997. Bayesian network classifiers. Mach Learn. 29, 131-163.

Fritz, P., 1984. An analytical solution for axisymmetric tunnel problems in elastoviscoplastic media. Int J Numer Anal Met. 8, 325-342.

Gao, F., Stead, D., Kang, H., 2015. Numerical simulation of squeezing failure in a coal mine roadway due to mining-induced stresses. Rock Mech Rock Eng. 48, 1635-1645.

Gao, W., 2015. Forecasting of rockbursts in deep underground engineering based on abstraction ant colony clustering algorithm. Nat Hazards. 76, 1625-1649.

Genuer, R., Poggi, J.-M., Tuleau-Malot, C., 2010. Variable selection using random forests. Pattern Recogn Lett. 31, 2225-2236.

Gioda, G., Cividini, A., 1996. Numerical methods for the analysis of tunnel performance in squeezing rocks. Rock Mech Rock Eng. 29, 171-193.

Goel, R., Jethwa, J., Paithankar, A., 1995. Indian experiences with Q and RMR systems. Tunn Undergr Sp Tech. 10, 97-109.

Goel, R., Singh, B., 2011. Engineering rock mass classification: tunnelling, foundations and landslides. Butterworth-Heinemann, Oxford, UK.

Grünwald, P. 2005. A tutorial introduction to the minimum description length principle. In: Grünwald, P., Myung, I.J.,Pitt, M.A. (eds.) Advances in Minimum Description Length: Theory and Applications. MIT Press, Cambridge, Massachusetts, USA.

Guelman, L., 2012. Gradient boosting trees for auto insurance loss cost modeling and prediction. Expert Syst Appl. 39, 3659-3667.

Guo, C., Zhang, Y., Deng, H., et al., 2011. Study on rock burst prediction in the deepburied tunnel at Gaoligong Mountain based on the rock proneness. Geotech Invest Surv. $39,8-13$.

Guo, D., Zhang, T., Li, Y., et al., 2008. Research on Rockburst Tendency and Its Preventive Measures of $800 \mathrm{~m}$ Deep Surrounding-Rock in Huxi Coal Mine. China Mining Magazine. 17, 50-54.

Guo, Y., Jiang, F., Application of comprehensive fuzzy evaluation in burst-proneness risk of coal seam. In: Qi, L.,Zhu, J.W. (eds) Second International Conference on Future Information Technology and Management Engineering, Fitme 2009, Los Alamitos, Ieee Computer Soc, pp 404-407.

Hall, M., Frank, E., Holmes, G., et al., 2009. The WEKA data mining software: an update. ACM SIGKDD explorations newsletter. 11, 10-18.

Haykin, S., 1998. Neural Networks: A Comprehensive Foundation. Prentice Hall PTR, New York, USA.

Heal, D., 2010. Observations and analysis of incidences of rockburst damage in underground mines. University of Western Australia. 
Heckerman, D., 1997. Bayesian networks for data mining. Data Min Knowl Disc. 1, 79-119.

Heckerman, D., Geiger, D., Chickering, D.M., 1995. Learning Bayesian Networks: the combination of knowledge and statistical data. Mach Learn. 20, 197-243.

Hoek, E., 2001. Big tunnels in bad rock. J Geotech Geoenviron. 127, 726-740.

Hoek, E., Brown, E.T., 1980. Underground excavations in rock. Institution of Mining and Metallurgy, London, UK.

Hoek, E., Guevara, R., 2009. Overcoming squeezing in the Yacambú-Quibor Tunnel, Venezuela. Rock Mech Rock Eng. 42, 389-418.

Hoek, E., Marinos, P., 2009. Tunnelling in overstressed rock. In: ISRM Regional Symposium-EUROCK 2009, Cavtat, Croatia.

Holmes, G., Donkin, A., Witten, I.H., WEKA: a machine learning workbench. In: Intelligent Information Systems, 1994. Proceedings of the 1994 Second Australian and New Zealand Conference on, pp 357-361.

Hornik, K., 2012. The comprehensive R archive network. Wiley Interdisciplinary Reviews: Computational Statistics. 4, 394-398.

Hornik, K., Buchta, C., Zeileis, A., 2009. Open-source machine learning: R meets Weka. Computation Stat. 24, 225-232.

Hosmer Jr, D.W., Lemeshow, S., Sturdivant, R.X., 2013. Applied logistic regression. John Wiley \& Sons, Hoboken, New Jersey, USA.

Hou, F., Wang, M., 1989. Criterion and prevention measures on rockburst in circular tunnel. In: Chinese Society for Rock Mechanics and Engineering. Proceedings of the 2th National Rock Mechanics and Engineering, Knowledge Press, Beijing, pp 195-201.

Huang, H., Zhang, J., Zhang, L., 2012. Bayesian network for characterizing model uncertainty of liquefaction potential evaluation models. KSCE Journal of Civil Engineering. 16, 714-722.

Huang, Q., Wang, N., 1999. Analysis of dynamic disturbance on rock burst. Bull Eng Geol Environ. 57, 281-284.

Hudson, J., 1992. Rock engineering systems: Theory and practice. Ellis Horwood Ltd, London, UK.

Hudson, J.A., A review of Rock Engineering Systems (RES) applications over the last 20 years. In: Feng, X., Hudson, J.A.,Tan, F. (eds) 3rd ISRM SINOROCK Symposium Shanghai, China, CRC Press, pp 419-424.

Hudson, J.A., Harrison, J.P., 1992. A new approach to studying complete rock engineering problems. Q J Eng Geol Hydroge. 25, 93-105. 
Itasca, 2012. FLAC - fast Lagrangian analysis of continua. Itasca Consulting Group Inc.

Itasca, 2014. PFC2D—particle flow code. Itasca Consulting Group Inc.

Jethwa, J.L., Singh, B., Singh, B. 1984. Estimation of ultimate rock pressure for tunnel linings under squeezing rock conditions - a new approach. In: Brown, E.T.,Hudson, J.A. (eds.) Design and Performance of Underground Excavations: ISRM Symposium Cambridge, U.K., 3-6 September 1984, 231-238.

Jia, R., Liu, C., Sun, H., et al., 2015. A situation assessment method for rock burst based on multi-agent information fusion. Comput Electr Eng. 45, 22-32.

Jimenez, R., Recio, D., 2011. A linear classifier for probabilistic prediction of squeezing conditions in Himalayan tunnels. Eng Geol. 121, 101-109.

Jimenez, R., Sitar, N., 2006. Inference of discontinuity trace length distributions using statistical graphical models. Int J Rock Mech Min. 43, 877-893.

Kaiser, P., MacCreath, D., Tannant, D., 1996. Canadian rockburst support handbook : prepared for sponsors of the Canadian rockburst research program 1990 - 1995. Geomechanics Research Centre, Sudbury, Canada.

Kaiser, P.K., Cai, M., 2012. Design of rock support system under rockburst condition. Journal of Rock Mechanics and Geotechnical Engineering. 4, 215-227.

Kaiser, P.K., Maloney, S.M., 1997. Scaling laws for the design of rock support. Pure Appl Geophys. 150, 415-434.

Kaiser, P.K., Tannant, D.D., McCreath, D.R., et al. 1992. Rockburst damage assessment procedure. In: Kaiser, P.,McCreath, D. (eds.) Rock Support in Mining and Underground Construction. CRC Press, Ontario, Canada, 639-647.

Karatzoglou, A., Smola, A., Hornik, K., 2016. kernlab: Kernel-Based Machine Learning Lab.

Kidybiński, A., 1981. Bursting liability indices of coal. International Journal of Rock Mechanics and Mining Sciences \& Geomechanics Abstracts. 18, 295-304.

Kohavi, R., A study of cross-validation and bootstrap for accuracy estimation and model selection. In: Proceedings of the 14th international joint conference on Artificial intelligence-Volume 2, Montreal, Canada, Morgan Kaufmann Publishers, Massachusetts, United States, pp 1137-1143.

Korb, K.B., Nicholson, A.E., 2010. Bayesian artificial intelligence. CRC press, London.

Kuhn, M., Johnson, K., 2013. Applied predictive modeling. Springer, New York.

Landis, J.R., Koch, G.G., 1977. The measurement of observer agreement for categorical data. Biometrics, 159-174.

Lauritzen, S.L., Spiegelhalter, D.J., 1988. Local computations with probabilities on 
graphical structures and their application to expert systems. Journal of the Royal Statistical Society. Series B (Methodological), 157-224.

Li, B., Liu, Y., 2015. Determination of classification of rock burst risk based on random forest approach and its application. Science \& Technology Review. 33, 57-62.

Li, N., Feng, X., Jimenez, R., 2017a. Predicting rock burst hazard with incomplete data using Bayesian networks. Tunn Undergr Sp Tech. 61, 61-70.

Li, N., Jimenez, R., 2017. A logistic regression classifier for long-term probabilistic prediction of rock burst hazard. Nat Hazards.

Li, N., Jimenez, R., Feng, X., 2017b. The Influence of Bayesian Networks Structure on Rock Burst Hazard Prediction with Incomplete Data. Procedia Engineering. 191, 206214.

Li, S., Zhao, H., Ru, Z., 2012. Deformation prediction of tunnel surrounding rock mass using CPSO-SVM model. J. Cent. South Univ. 19, 3311-3319.

Li, X., Wang, E., Li, Z., et al., 2016. Rock burst monitoring by integrated microseismic and electromagnetic radiation methods. Rock Mech Rock Eng. 49, 4393-4406.

Li, Y., Fu, Y., Li, H., et al., The Improved Training Algorithm of Back Propagation Neural Network with Self-adaptive Learning Rate. In: 2009 International Conference on Computational Intelligence and Natural Computing, pp 73-76.

Li, Z., Dou, L., Cai, W., et al., 2014. Investigation and analysis of the rock burst mechanism induced within fault-pillars. Int J Rock Mech Min. 70, 192-200.

Liang, Z., 2004. Study on the Prediction and Prevention of Rockburst in the Diversion Tunnel of Jinping II Hydropower Station. M.Sc, Chengdu University of Technology.

Liaw, A., Wiener, M., 2002. Classification and regression by random forest. R News: The Newsletter of the R Project. 2/3, 18.

Liu, L., Cheng, Y., 2016. Efficient system reliability analysis of soil slopes using multivariate adaptive regression splines-based Monte Carlo simulation. Comput Geotech. 79, 41-54.

Liu, Z., Shao, J., Xu, W., et al., 2013. Prediction of rock burst classification using the technique of cloud models with attribution weight. Nat Hazards. 68, 549-568.

Liu, Z.J., Yuan, Q.P., Li, J.L., 2008. Application of fuzzy probability model to prediction of classification of rockburst intensity. Chinese Journal of Rock Mechanics and Engineering. 27, 3095-3103.

Loozen, G., Ozcelik, O., Boon, N., et al., 2014. Inter-bacterial correlations in subgingival biofilms: a large-scale survey. J Clin Periodontol. 41, 1-10.

Lu, C., Liu, G., Liu, Y., et al., 2015. Microseismic multi-parameter characteristics of rockburst hazard induced by hard roof fall and high stress concentration. Int J Rock Mech Min. 76, 18-32. 
Margaritis, D., 2003. Learning Bayesian network model structure from data. Carnegie Mellon University.

Matías, J.M., Vaamonde, A., Taboada, J., et al., 2004. Support vector machines and gradient boosting for graphical estimation of a slate deposit. Stoch Env Res Risk A. 18, 309-323.

Medina-Cetina, Z., Nadim, F., 2008. Stochastic design of an early warning system. Georisk: Assessment and Management of Risk for Engineered Systems and Geohazards. 2, 223-236.

Meng, X., Bradley, J., Yavuz, B., et al., 2016. Mllib: Machine learning in apache spark. J Mach Learn Res. 17, 1-7.

Metz, C.E., 1978. Basic principles of ROC analysis. Semin Nucl Med. 8, 283-298.

Micheletti, N., Foresti, L., Robert, S., et al., 2014. Machine learning feature selection methods for landslide susceptibility mapping. Mathematical Geosciences. 46, 33-57.

Milborrow, S., 2011. earth: Multivariate Adaptive Regression Splines.

Mirzavand, M., Khoshnevisan, B., Shamshirband, S., et al., 2015. Evaluating groundwater level fluctuation by support vector regression and neuro-fuzzy methods: a comparative study. Nat Hazards, 1-15.

Mitchell, T. 2015. Generative and Discriminative Classifiers: Naive Bayes and Logistic Regression. https://www.cs.cmu.edu/ tom/mlbook/NBayesLogReg.pdf.

Moguerza, J.M., Muñoz, A., 2006. Support vector machines with applications. Stat Sci. 21, 322-336.

Mutke, G., Dubinski, J., Lurka, A., 2015. New criteria to assess seismic and rock burst hazard in coal mines. Arch. Min. Sci. 60, 743-760.

Naghadehi, M.Z., Jimenez, R., KhaloKakaie, R., et al., 2011. A probabilistic systems methodology to analyze the importance of factors affecting the stability of rock slopes. Eng Geol. 118, 82-92.

Naghadehi, M.Z., Jimenez, R., KhaloKakaie, R., et al., 2013. A new open-pit mine slope instability index defined using the improved rock engineering systems approach. Int J Rock Mech Min. 61, 1-14.

Natekin, A., Knoll, A., 2013. Gradient Boosting Machines, A Tutorial. Frontiers in Neurorobotics. 7.

Ng, A.Y., Jordan, M.I., On discriminative vs. generative classifiers: A comparison of logistic regression and naive bayes. In: Advances in neural information processing systems, pp 841-848.

Nielsen, T.D., Jensen, F.V., 2009. Bayesian networks and decision graphs. Springer Science \& Business Media, New York, USA. 
NorsysSoftware, 1998. Norsys Software Corporation, Vancouver, BC, Canada.

Ortlepp, W.D., Stacey, T.R., 1994. Rockburst mechanisms in tunnels and shafts. Tunn Undergr Sp Tech. 9, 59-65.

Pan, Y., Li, Z., 2002. The analytic analysis stability of rock structure in mine. In: Proceedings of National Conference on Solid, Mechanics, Dalian University of Technology Press, Dalian, China.

Pan, Y., Li, Z., 2005. Analysis of rock structure stability in coal mines. Int J Numer Anal Met. 29, 1045-1063.

Pearce, J., Ferrier, S., 2000. Evaluating the predictive performance of habitat models developed using logistic regression. Ecol Model. 133, 225-245.

Pearl, J., 1986. A constraint propagation approach to probabilistic reasoning. In: Proceedings of the First Annual Conference on Uncetainty in Aritificial Intelligence (UAI-85), Corvallis, Oregon, AUAI Press, pp 31-42.

Pearl, J., 2014. Probabilistic reasoning in intelligent systems: networks of plausible inference. Morgan Kaufmann, San Francisco, USA.

Pearl, J., Verma, T.S., 1995. A theory of inferred causation. Studies in Logic and the Foundations of Mathematics. 134, 789-811.

Peng, Q., Qian, A., Xiao, Y., 2010. Research on prediction system for rockburst based on artificial intelligence application methods. Journal of Sichuan University: Engineering Science Edition. 42, 18-24.

Peng, Y., Peng, K., Zhou, J., et al., 2014. Prediction of classification of rock burst risk based on genetic algorithms with SVM. In: 2nd International Conference on Machinery, Materials Science and Energy Engineering (ICMMSEE), Changsha, China, Trans Tech Publications Ltd, Zurich, Switzerland, pp 383-389.

Pfanzagl, J., 1994. Parametric statistical theory. Walter de Gruyter, New York, USA.

Qian, Q., 2014. Definition, mechanism, classification and quantitative forecast model for rockburst and pressure bump. Rock and Soil Mechanics. 35, 1-6.

Qin, S., Chen, J., Wang, Q., 2009. Research on rockburst prediction with extenics evaluation based on rough set. In: Tang, C.A.E. (ed) Proceedings of the 13th International Symposium on Rockburst and Seismicity in Mines, Dalian, China, Rinton Press, pp 937-944.

R Core Team, R., 2014. R: A language and environment for statistical computing. R Foundation for Statistical Computing. Vienna, Austria.

Recio-Gordo, D., Jimenez, R., 2012. A probabilistic extension to the empirical ALPS and ARMPS systems for coal pillar design. Int J Rock Mech Min. 52, 181-187. 
Recio Gordo, D., 2014. Aplicación de la regresión logística en la predicción empírica de fenómenos complejos en obras subterráneas : squeezing y rotura de pilares de carbón. Doctoral, E.T.S.I Caminos, Canales y Puertos, Universidad Politécnica de Madrid.

Ridgeway, G., 2004. gbm: Generalized Boosted Regression Models.

Ripley, B., Venables, W., 2016. nnet: Feed-Forward Neural Networks and Multinomial Log-Linear Models.

Roever, C., Raabe, N., Luebke, K., et al., 2014. klaR: Classification and visualization. Fakultaet Statistik, Technische Universitaet Dortmund.

Rosasco, L., Vito, E.D., Caponnetto, A., et al., 2004. Are Loss Functions All the Same? Neural Comput. 16, 1063-1076.

Russell, S.J., Norvig, P., 2003. Artificial Intelligence: A Modern Approach. Pearson Education, New Jersey, USA.

Russenes, B., 1974. Analysis of rock spalling for tunnels in steep valley sides. Norwegian Institute of Technology.

Ryder, J., 1988. Excess shear stress in the assessment of geologically hazardous situations. J S Afr I Min Metall. 88, 27-39.

Salazar, F., Toledo, M., Oñate, E., et al., 2015. An empirical comparison of machine learning techniques for dam behaviour modelling. Struct Saf. 56, 9-17.

Schubert, M., Høj, N.P., Ragnøy, A., et al., 2012. Risk assessment of road tunnels using Bayesian Networks. Procedia - Social and Behavioral Sciences. 48, 2697-2706.

Schubert, W., 1996. Dealing with squeezing conditions in Alpine tunnels. Rock Mech Rock Eng. 29, 145-153.

Schwarz, G., 1978. Estimating the dimension of a model. Ann. Statist. 6, 461-464.

Shafiei, A., Parsaei, H., Dusseault, M., 2012. Rock squeezing prediction by a Support Vector Machine Classifier. In: 46th US Rock Mechanics/Geomechanics Symposium, Chicago, Illinois.

Shalabi, F.I., 2005. FE analysis of time-dependent behavior of tunneling in squeezing ground using two different creep models. Tunn Undergr Sp Tech. 20, 271-279.

Shan, Z., Yan, P., 2010. Management of rock bursts during excavation of the deep tunnels in Jinping II Hydropower Station. Bull Eng Geol Environ. 69, 353-363.

Shannon, C.E., Weaver, W., 1998. The mathematical theory of communication. University of Illinois press, Urbana, USA.

Shrestha, G.L., 2006. Stress induced problems in Himalayan tunnels with special reference to squeezing. Norwegian University of Science and Technology.

Singh, B., Goel, R., Jethwa, J., et al., 1997. Support pressure assessment in arched 
underground openings through poor rock masses. Eng Geol. 48, 59-81.

Singh, B., Jethwa, J., Dube, A., et al., 1992. Correlation between observed support pressure and rock mass quality. Tunn Undergr Sp Tech. 7, 59-74.

Singh, S., 1988. Burst energy release index. Rock Mech Rock Eng. 21, 149-155.

Singla, P., Domingos, P., Discriminative training of Markov logic networks. In: AAAI, pp 868-873.

Smola, A.J., Schölkopf, B., 2004. A tutorial on support vector regression. Stat Comput. 14, 199-222.

Song, Y., Gong, J., Gao, S., et al., 2012. Susceptibility assessment of earthquakeinduced landslides using Bayesian network: A case study in Beichuan, China. Comput Geosci-uk. 42, 189-199.

Sonnenburg, S., Henschel, S., Widmer, C., et al., 2010. The SHOGUN machine learning toolbox. J Mach Learn Res. 11, 1799-1802.

Sousa, R.L., Einstein, H.H., 2012. Risk analysis during tunnel construction using Bayesian Networks: Porto Metro case study. Tunn Undergr Sp Tech. 27, 86-100.

Špačková, O., Šejnoha, J., Straub, D., 2013. Probabilistic assessment of tunnel construction performance based on data. Tunn Undergr Sp Tech. 37, 62-78.

Spirtes, P., Glymour, C., 1991. An algorithm for fast recovery of sparse causal graphs. Soc Sci Comput Rev. 9, 62-72.

Sterpi, D., Gioda, G., 2007. Visco-Plastic behaviour around advancing tunnels in squeezing rock. Rock Mech Rock Eng. 42, 319-339.

Sulem, J., Panet, M., Guenot, A., 1987. Closure analysis in deep tunnels. International Journal of Rock Mechanics and Mining Sciences \& Geomechanics Abstracts. 24, 145154.

Suman, S., Khan, S.Z., Das, S.K., et al., 2016. Slope stability analysis using artificial intelligence techniques. Nat Hazards. 84, 727-748.

Sun, H., Li, S., Qiu, D., et al., 2009. Application of extensible comprehensive evaluation to rockburst prediction in a relative shallow chamber. Proc., RaSiM7 (2009): Controlling Seismic Hazard and Sustainable Development of Deep Mines, CA Tang, ed., Rinton Press, Princeton, NJ, 777-784.

Ticehurst, J.L., Letcher, R.A., Rissik, D., 2008. Integration modelling and decision support: A case study of the Coastal Lake Assessment and Management (CLAM) Tool. Math Comput Simulat. 78, 435-449.

Tuler, F., Butcher, B., 1968. A criterion for the time dependence of dynamic fracture. International Journal of Fracture Mechanics. 4, 431-437.

Ulusay, R., Hudson, J.A., 2007. The complete ISRM suggested methods for rock characterization, testing and monitoring: 1974-2006. International Society for Rock 
Mechanics, Commission on Testing Methods, Ankara, Turkey.

Uusitalo, L., 2007. Advantages and challenges of Bayesian networks in environmental modelling. Ecol Model. 203, 312-318.

Verhulst, P., 1838. Notice surlaloique la population poursuitdans son accroissement. Correspondance.

Wang, J., Park, H., 2001. Comprehensive prediction of rockburst based on analysis of strain energy in rocks. Tunn Undergr Sp Tech. 16, 49-57.

Wang, Y., Li, W., Li, Q., et al., 1998. Method of fuzzy comprehensive evaluations for rockburst prediction. Chinese Journal of Rock Mechanics and Engineering. 17, 493501.

Wang, Y., Shang, Y., Sun, H., et al., 2010. Research and application of rockburst intensity prediction model based on entropy coefficient and ideal point method. Journal of China Coal Society. 2, 014.

Xiao, X., 2005. A study on the prediction and prevention of rockburst traffic tunnel of Jinping II hydropower station. Chengdu Univ. of Technology.

Xie, H., Pariseau, W.G., 1993. Fractal character and mechanism of rock bursts. International Journal of Rock Mechanics and Mining Sciences \& Geomechanics Abstracts. 30, 343-350.

Xu, Y., Zhang, L., Jia, J., 2011. Diagnosis of embankment dam distresses using Bayesian networks. Part II. Diagnosis of a specific distressed dam. Can Geotech J. 48, 1645-1657.

Yang, Y., Zhang, Q., 1998. The application of neural networks to rock engineering systems (RES). Int J Rock Mech Min. 35, 727-745.

Yassaghi, A., Salari-Rad, H., 2005. Squeezing rock conditions at an igneous contact zone in the Taloun tunnels, Tehran-Shomal freeway, Iran: a case study. Int J Rock Mech Min. 42, 95-108.

Yu, H., Liu, H., Lu, X., et al., 2009. Prediction method of rock burst proneness based on rough set and genetic algorithm. Journal of Coal Science and Engineering (China). $15,367-373$.

Zhang, M., Shimada, H., Sasaoka, T., et al., 2013. Seismic energy distribution and hazard assessment in underground coal mines using statistical energy analysis. Int $\mathbf{J}$ Rock Mech Min. 64, 192-200.

Zhang, Q., Wang, W., Liu, T., 2011. Prediction of Rock Bursts Based on Particle Swarm Optimization-BP Neural Network. Journal of China Three Gorges University. 33, 41$45,56$.

Zhang, W., Goh, A.T.C., 2016. Multivariate adaptive regression splines and neural network models for prediction of pile drivability. Geoscience Frontiers. 7, 45-52. 
Zhang, Z., Gao, F., Shang, X., 2014. Rock burst proneness prediction by acoustic emission test during rock deformation. J. Cent. South Univ. 21, 373-380.

Zhao, D., Han, C., Yan, H., et al., 2007. The coal and rock physics mechanics quality mensuration and impact orientation estimate of Guantai coal Mine. Coal. 9, 7.

Zhou, J., Li, X., Mitri, H.S., 2015. Comparative performance of six supervised learning methods for the development of models of hard rock pillar stability prediction. Nat Hazards. 79, 291-316.

Zhou, J., Li, X., Mitri, H.S., 2016a. Classification of rockburst in underground projects: comparison of ten supervised learning methods. J Comput Civil Eng. 30, 04016003.

Zhou, J., Li, X., Shi, X., 2012. Long-term prediction model of rockburst in underground openings using heuristic algorithms and support vector machines. Safety Sci. 50, 629644.

Zhou, J., Shi, X., Du, K., et al., 2017. Feasibility of Random-Forest approach for prediction of ground settlements induced by the construction of a shield-driven tunnel. International Journal of Geomechanics. 17.

Zhou, J., Shi, X., Huang, R., et al., 2016b. Feasibility of stochastic gradient boosting approach for predicting rockburst damage in burst-prone mines. T Nonferr Metal Soc. 26, 1938-1945.

Zhou, K., Lin, Y., Deng, H., et al., 2016c. Prediction of rock burst classification using cloud model with entropy weight. T Nonferr Metal Soc. 26, 1995-2002.

Zhu, W., Li, S., Li, S., et al., 2003. Systematic numerical simulation of rock tunnel stability considering different rock conditions and construction effects. Tunn Undergr Sp Tech. 18, 531-536.

Zubelewicz, A., Mroz, Z., 1983. Numerical-Simulation of rock Burst processes treated as problems of dynamic instability. Rock Mech Rock Eng. 16, 253-274. 



\section{APPENDICES}

Appendix A Database of 135 case histories of rock burst for long-term prediction

Appendix B Database of 254 case histories of rock burst damage scales for shortterm prediction

Appendix C Database of 166 case histories of rock tunnel squeezing 


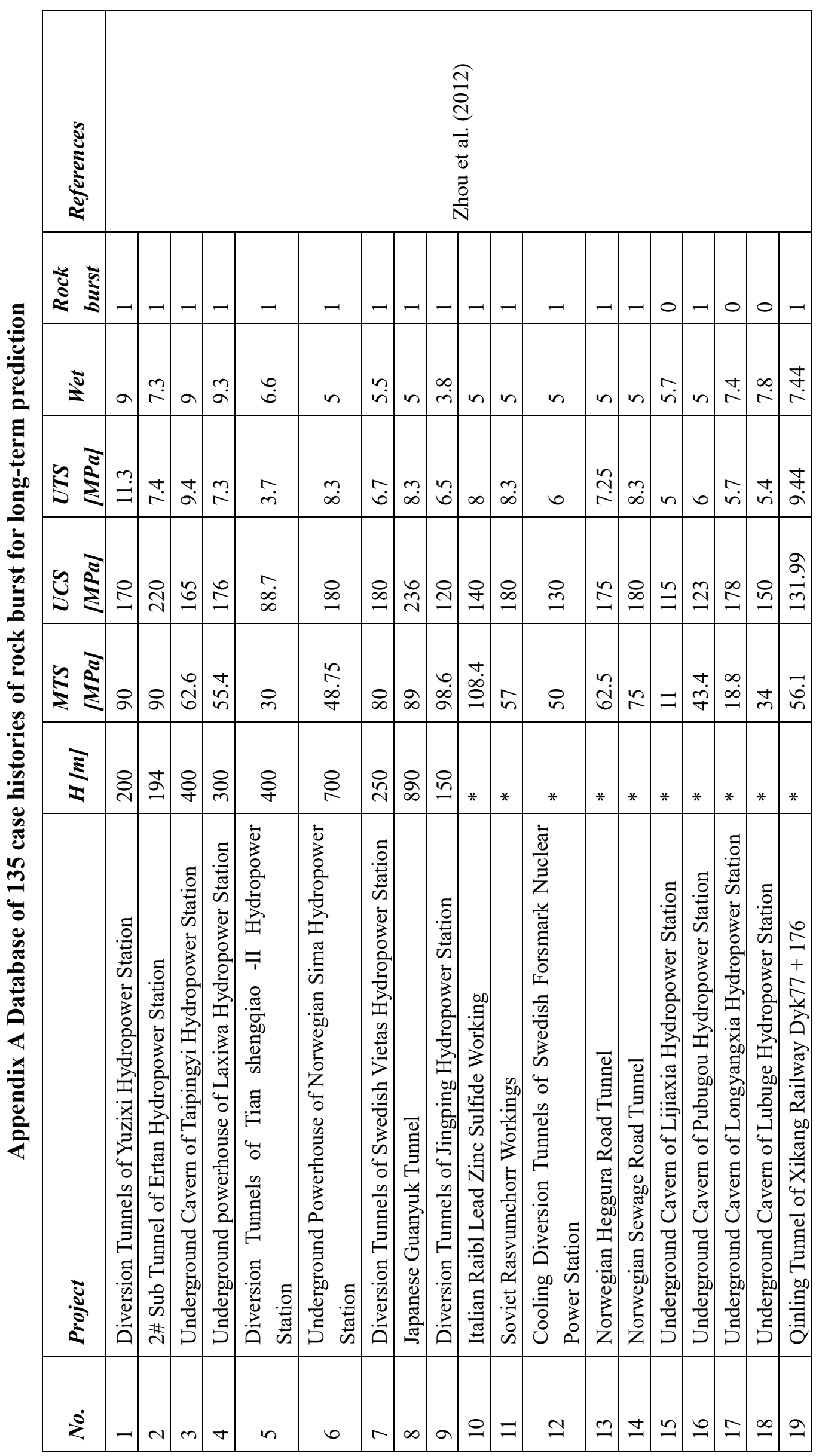




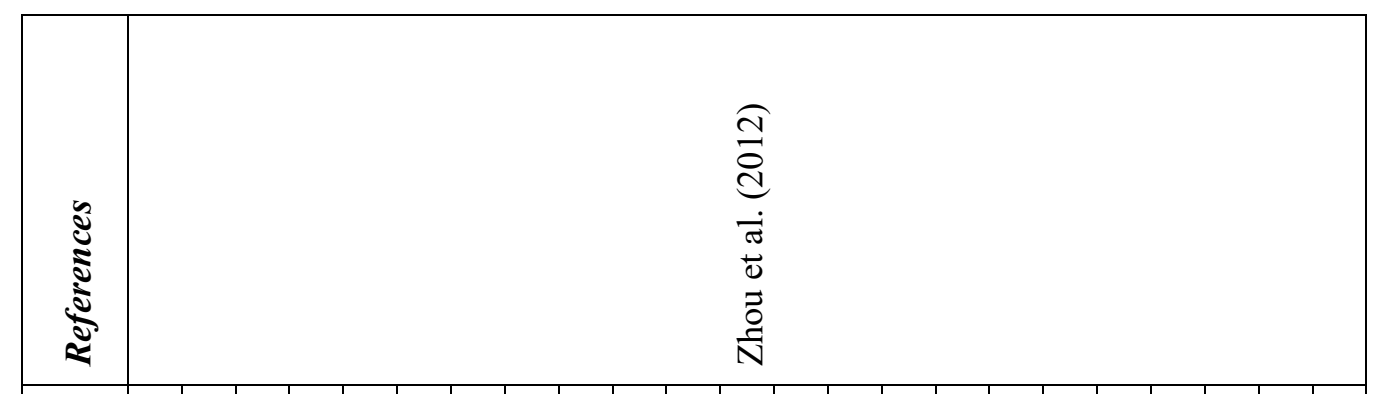

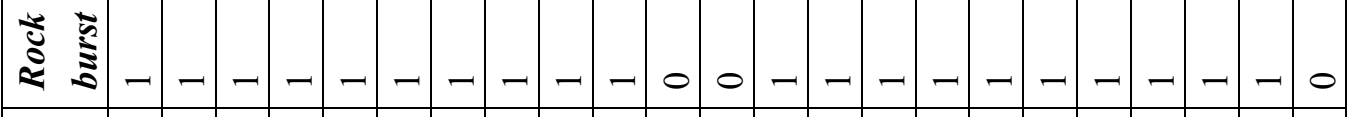

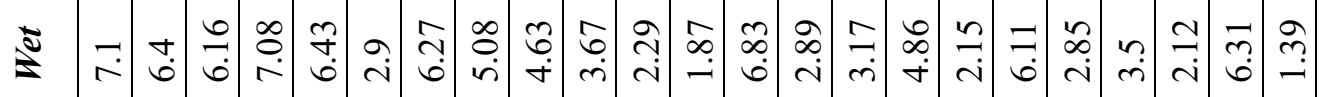

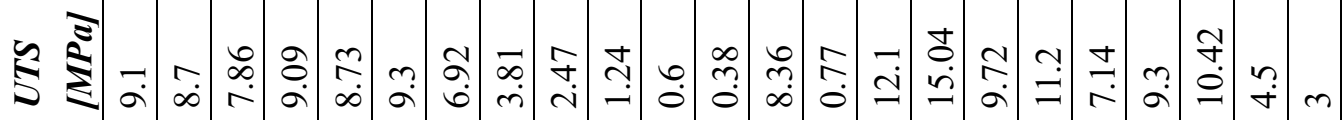

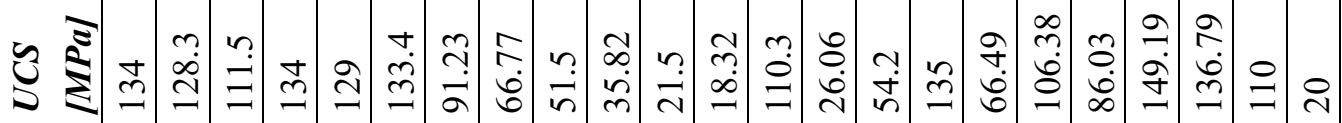

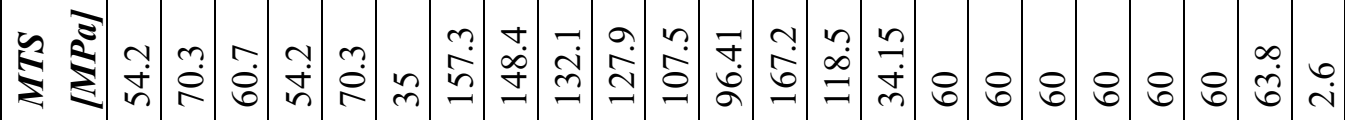

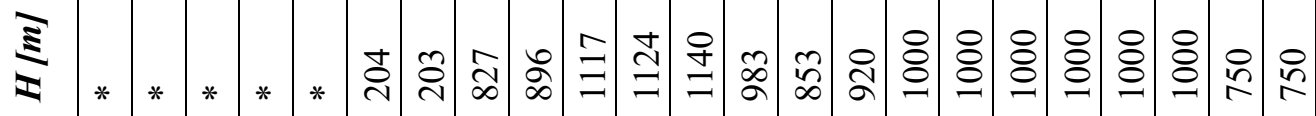

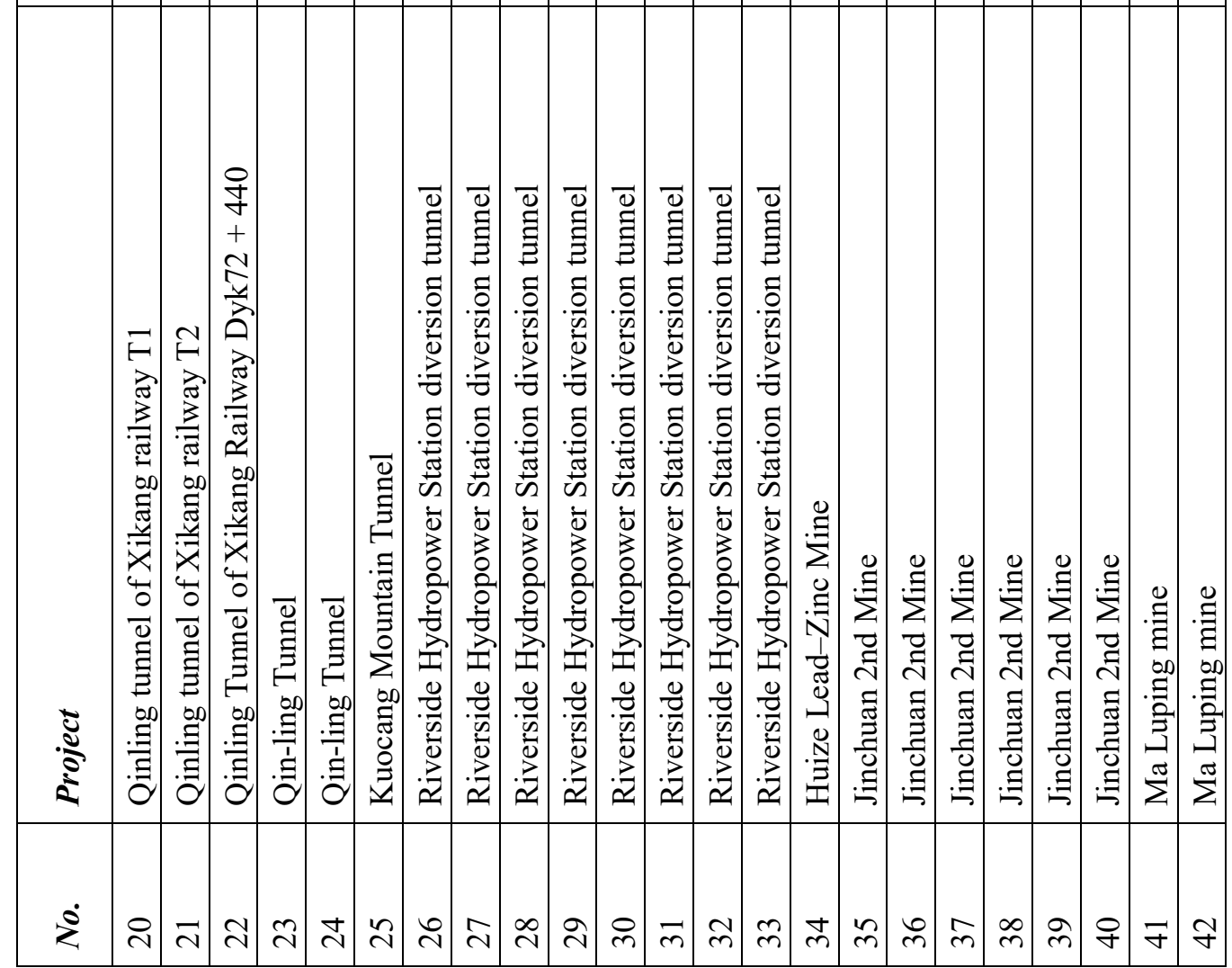




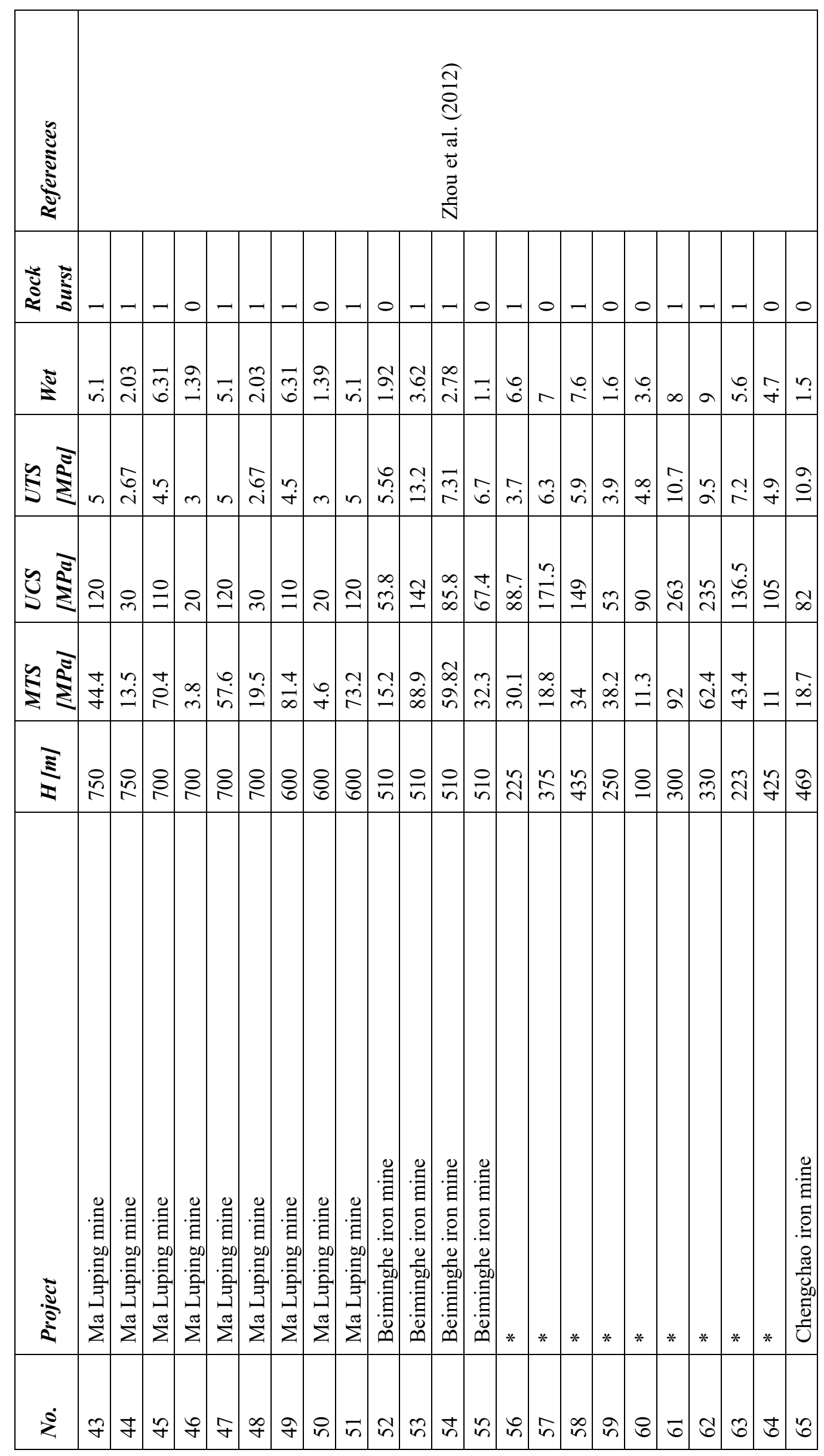




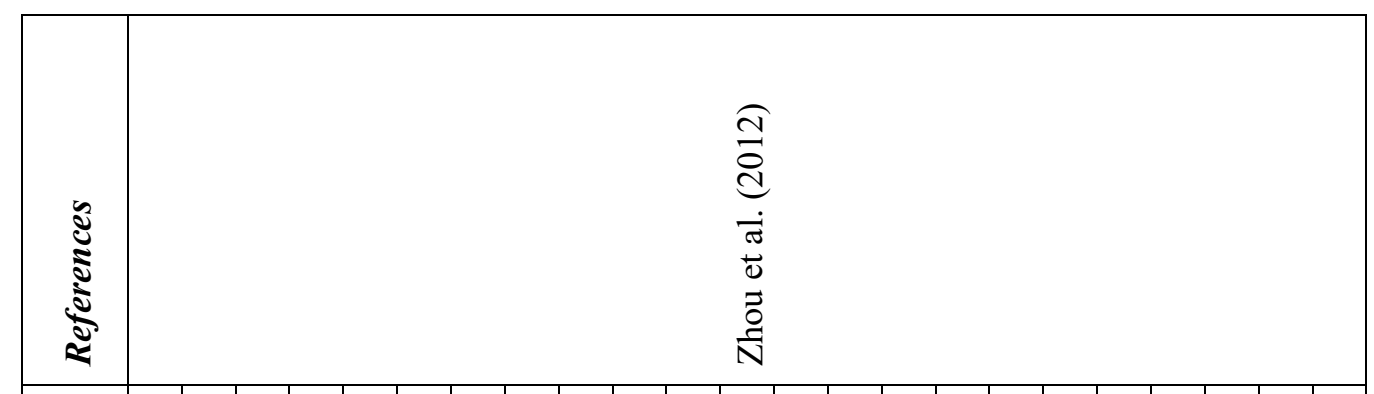

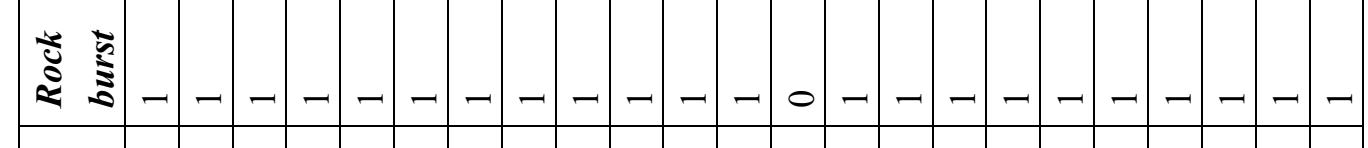

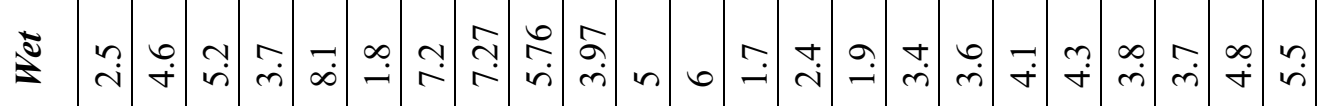

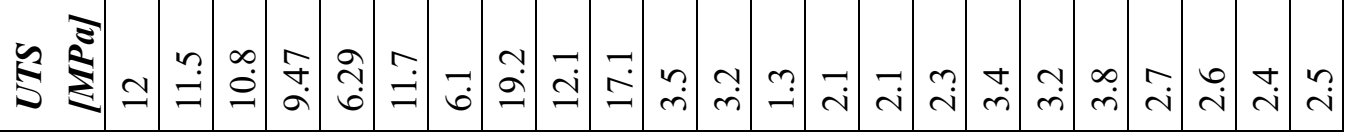

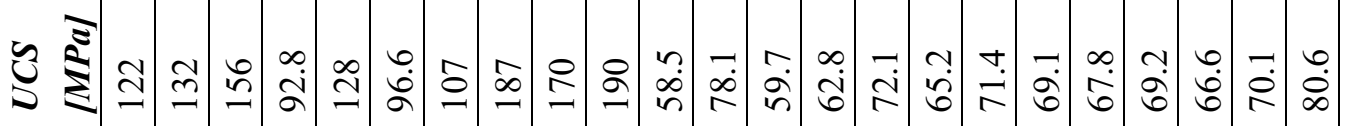

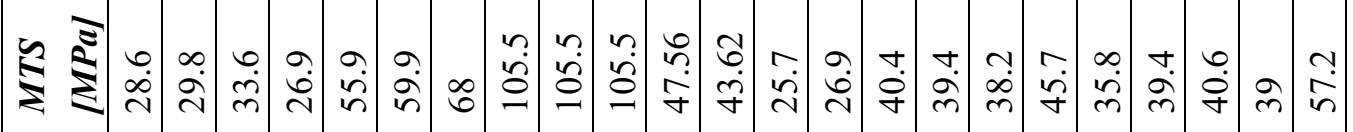

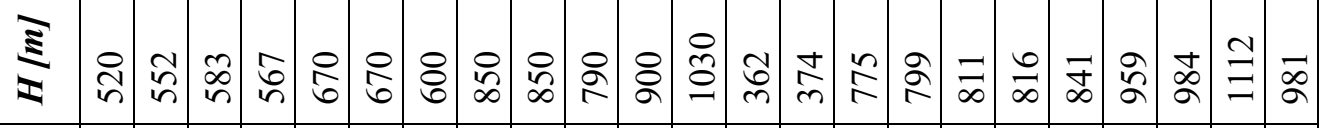

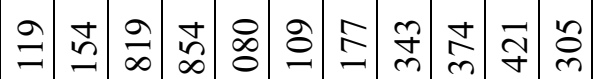

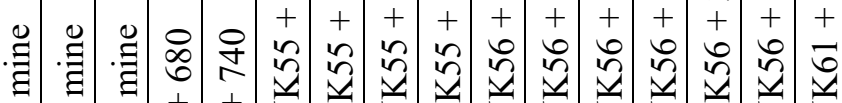

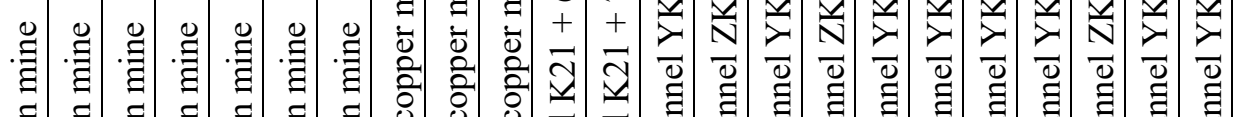

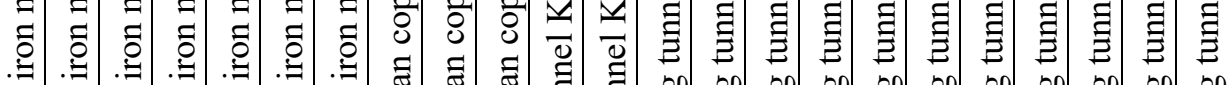

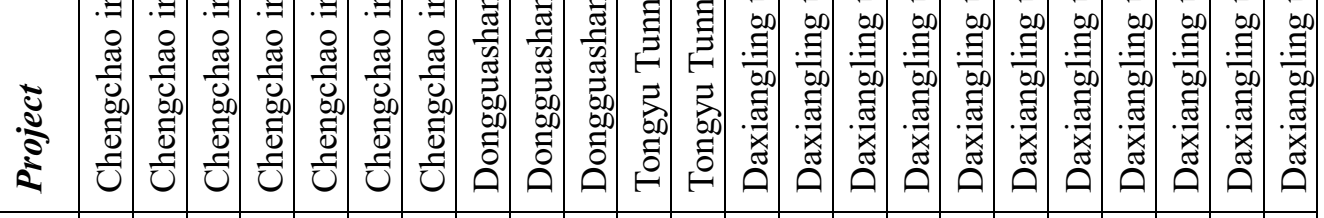

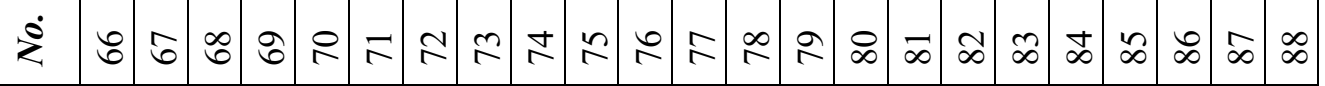




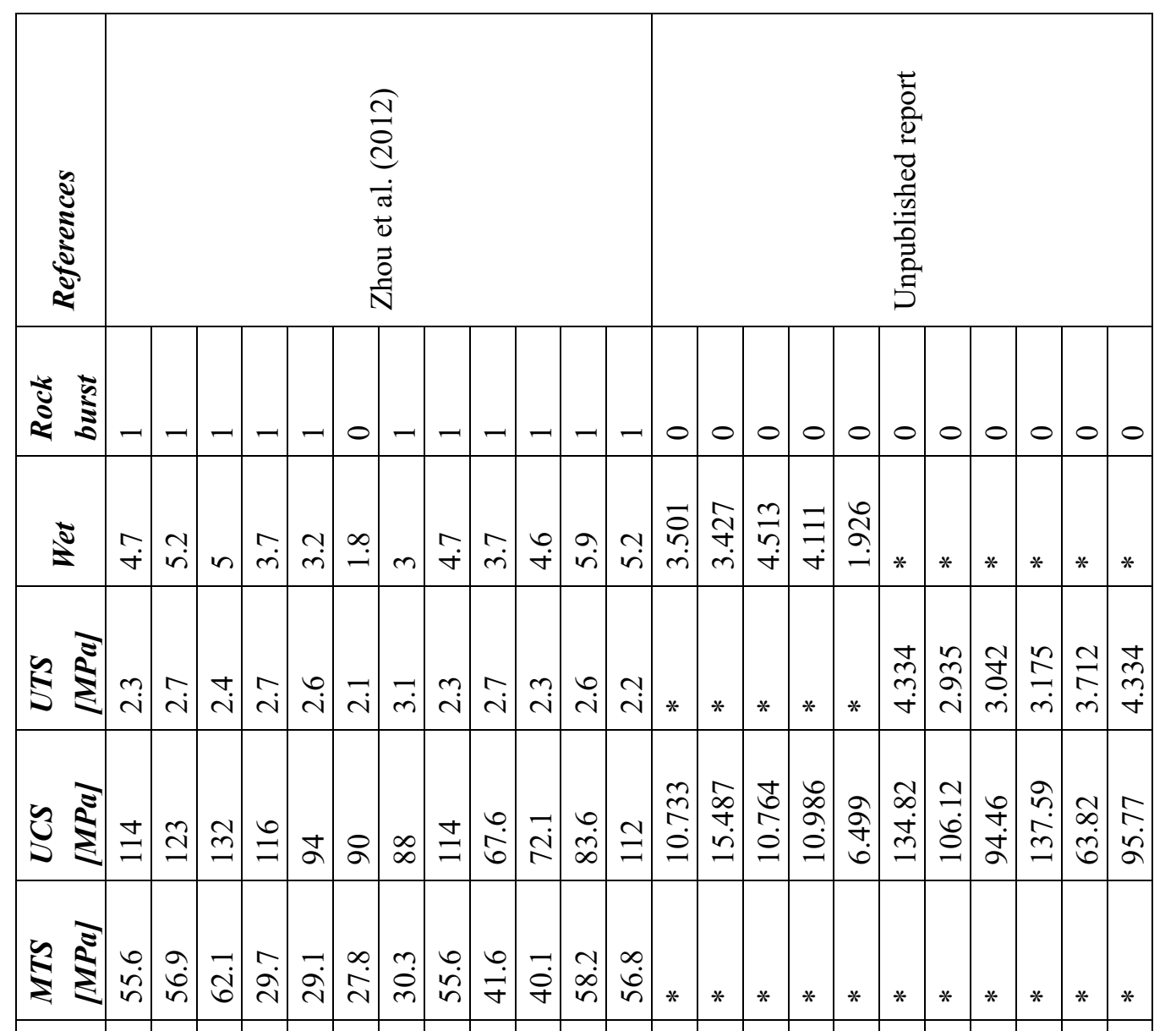

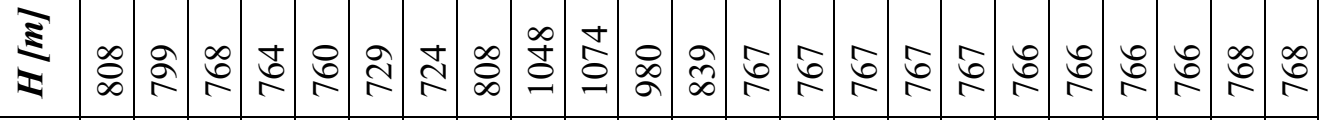

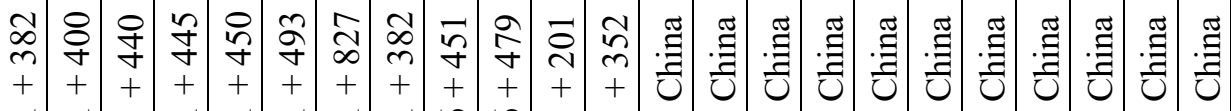

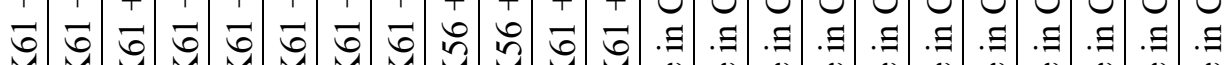

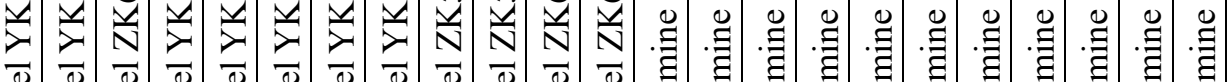

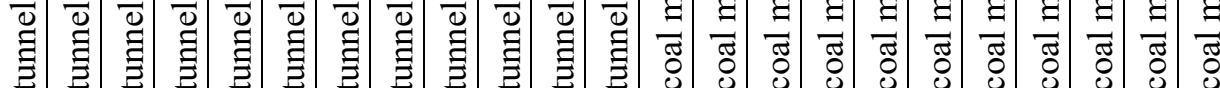
600 000 60.

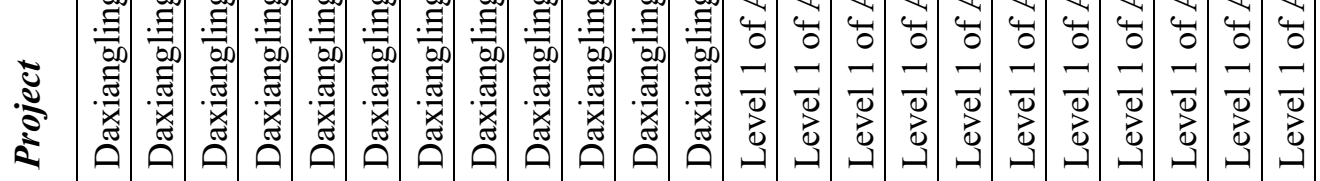

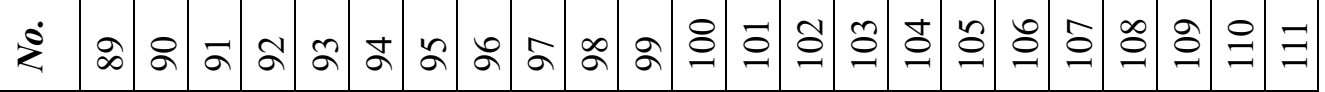




\begin{tabular}{|c|c|c|c|c|c|c|c|c|c|c|c|c|c|c|c|c|c|c|c|c|c|c|c|c|c|}
\hline 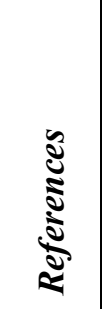 & & & & & & $=$ & & & & & & & 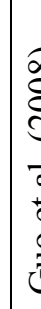 & & & 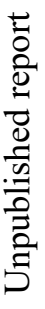 & & & 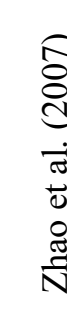 & & & & & 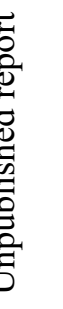 & \\
\hline 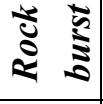 & 0 & 0 & 0 & 0 & $c$ & $c$ & & & P & 0 & 0 & & $c$ & & 0 & 0 & 0 & 0 & 0 & 0 & 0 & 0 & 0 & 0 & 0 \\
\hline$\Xi$ & $*$ & $*$ & ल) & б & i & * & & & 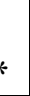 & $*$ & * & $*$ & 7 & 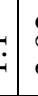 & $\tilde{\sigma}$ & * & $*$ & $\begin{array}{c}\hat{0} \\
\text { i }\end{array}$ & $\begin{array}{c}\hat{0} \\
i\end{array}$ & $\stackrel{\text { gे. }}{-}$ & 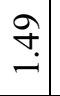 & $\stackrel{\infty}{-}$ & $\stackrel{\infty}{-}$ & 9 & * \\
\hline 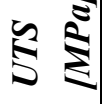 & $\begin{array}{l}\hat{0} \\
\stackrel{7}{+}\end{array}$ & $\begin{array}{l}n \\
\tilde{n} \\
i n\end{array}$ & $*$ & * & $*$ & c & & & $\vec{v}$ & 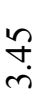 & $\begin{array}{l}\bar{J} \\
\gamma\end{array}$ & & $*$ & & So & 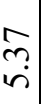 & $\stackrel{\infty}{n} \underset{\sim}{\sim}$ & $\begin{array}{l}\tilde{n} \\
n \\
n\end{array}$ & $\overrightarrow{\bar{i}}$ & $\begin{array}{l}\mathscr{D} \\
\stackrel{+}{+}\end{array}$ & $\begin{array}{l}\partial \\
\dot{i}\end{array}$ & * & $*$ & $*$ & 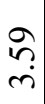 \\
\hline$\tilde{s}$ & $\begin{array}{l}J \\
\dot{\infty} \\
\infty\end{array}$ & 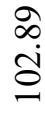 & $\vec{\sim}$ & حี & $\underset{\infty}{\infty}$ & z & & & כ. & $\hat{\alpha}$ & $\frac{\tilde{y}}{\sigma}$ & $\bar{s}$ & * & & â. & 年. & 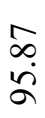 & $\begin{array}{l}\stackrel{+}{+} \\
\dot{n}\end{array}$ & 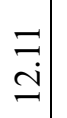 & $\begin{array}{l}\dot{v} \\
i \\
i\end{array}$ & 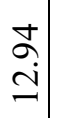 & $\begin{array}{l}0 \\
\dot{r}\end{array}$ & $\ddot{\nabla}$ & $\hat{\mathrm{i}}$ & $\begin{array}{l}\infty \\
\dot{\infty} \\
\dot{\infty}\end{array}$ \\
\hline$\stackrel{\Xi}{\Xi}$ & $*$ & $*$ & $*$ & * & * & * & & & 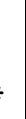 & * & & $*$ & * & & * & * & * & * & $*$ & $*$ & $*$ & $*$ & $*$ & $*$ & $*$ \\
\hline
\end{tabular}

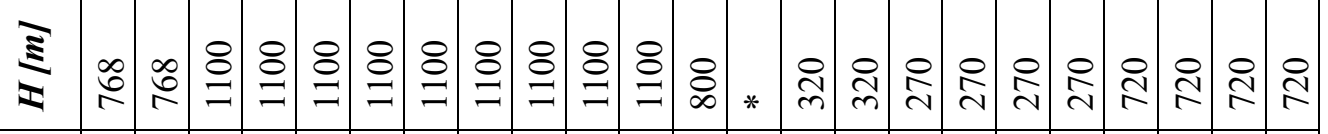

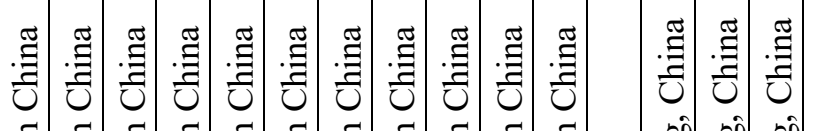

$. \Xi . \Xi . \Xi . \Xi . \Xi . \Xi . \Xi . \Xi . \Xi . \Xi . \Xi \quad$ of

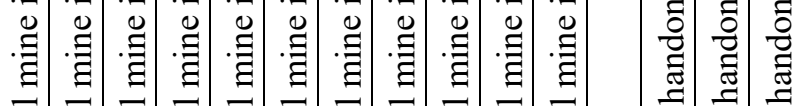

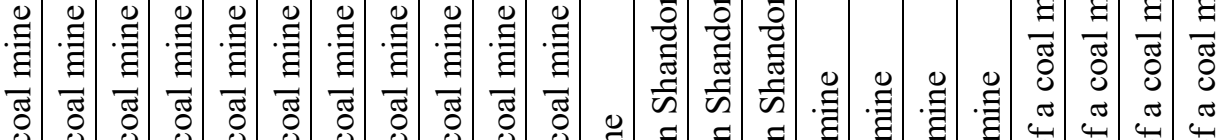

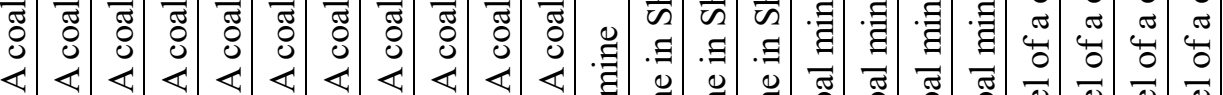

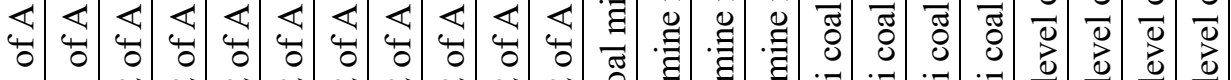

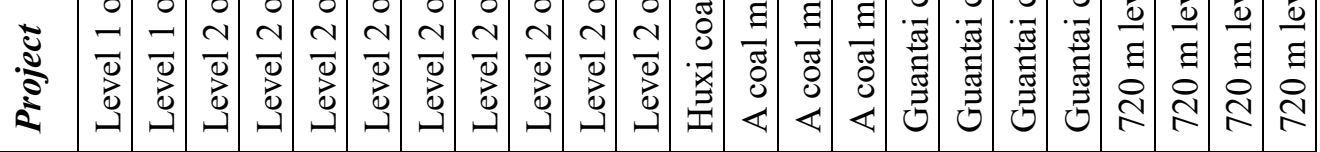

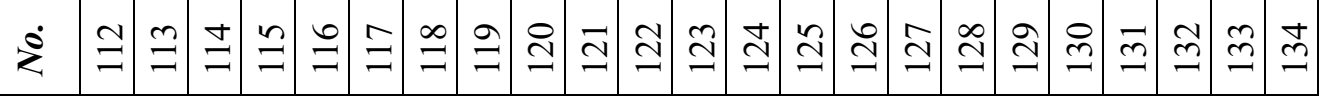




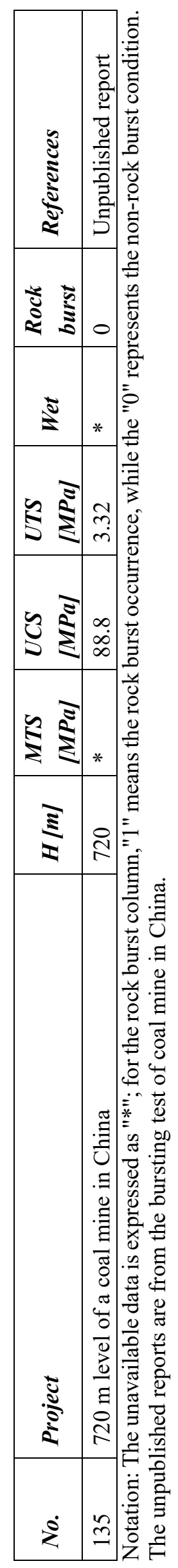




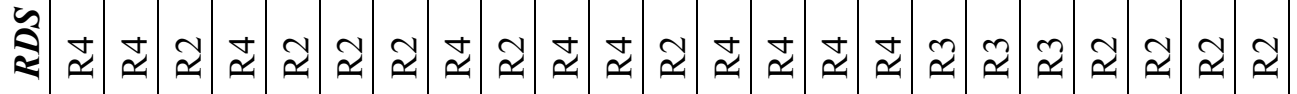

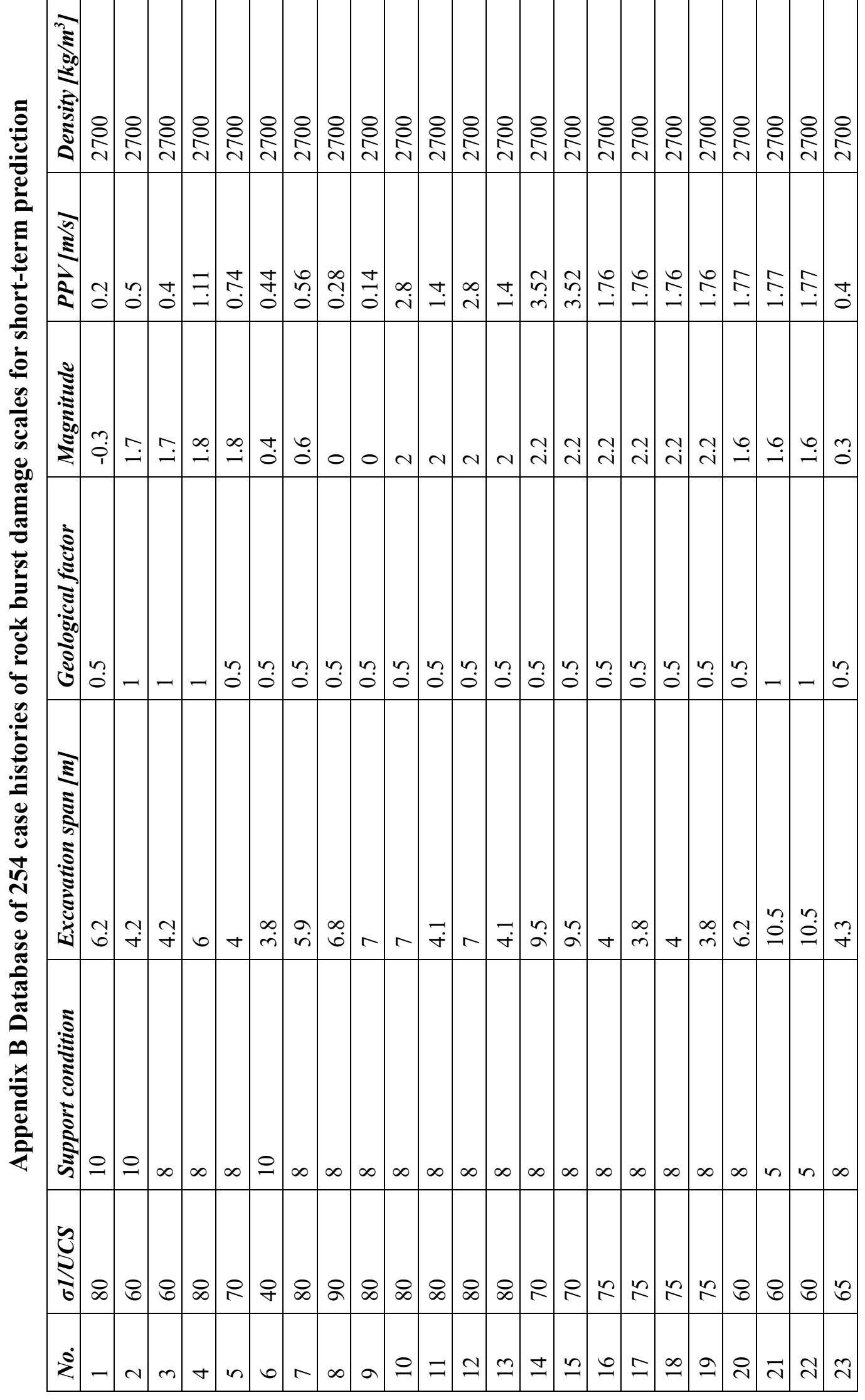




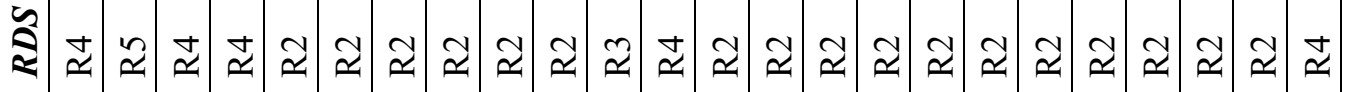

בิ

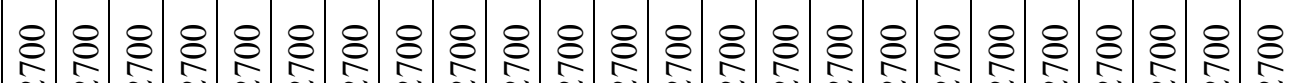

है

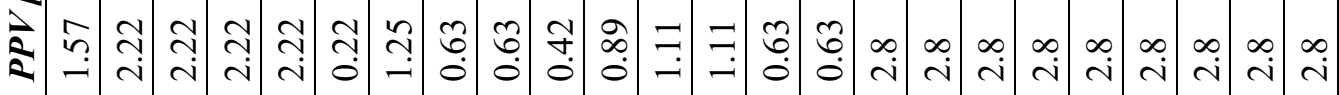

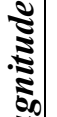

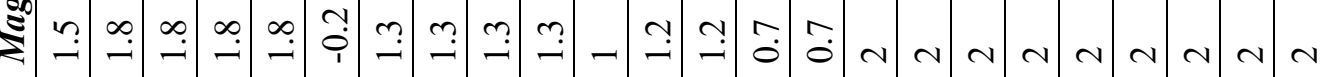

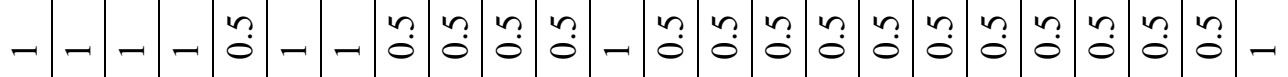

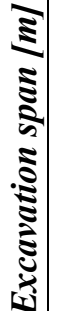

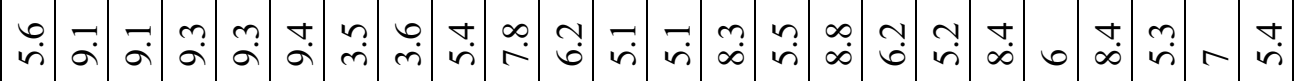

ב⿱艹

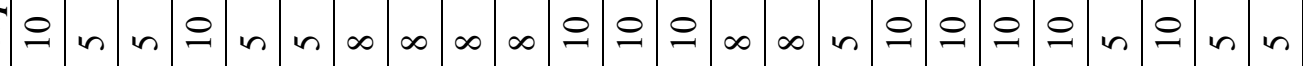

2

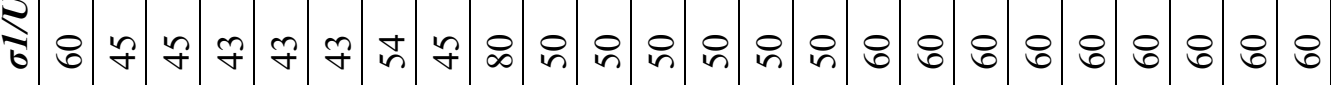

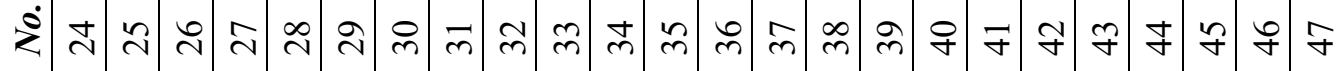




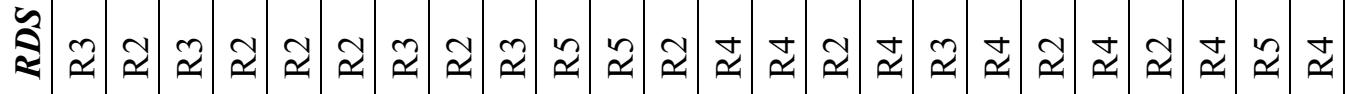

בิ

군

इ

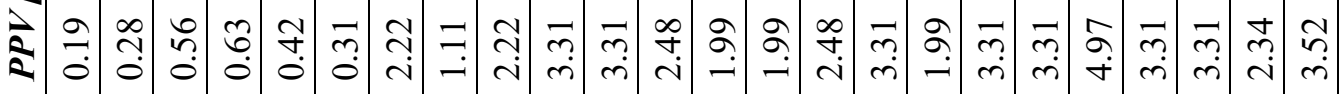

:

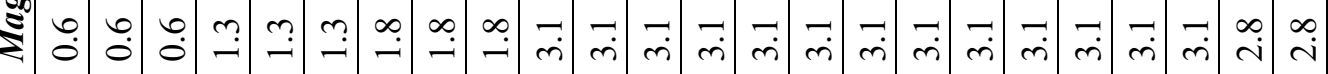

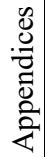

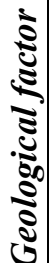

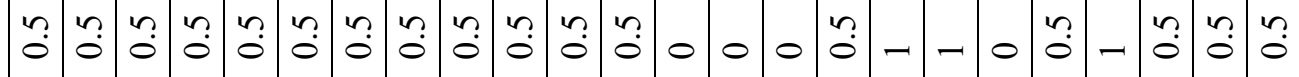

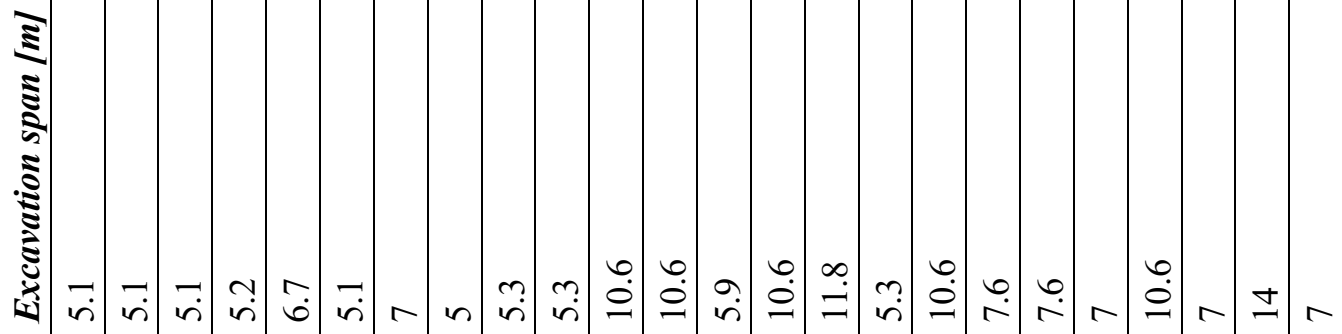

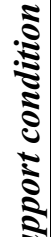

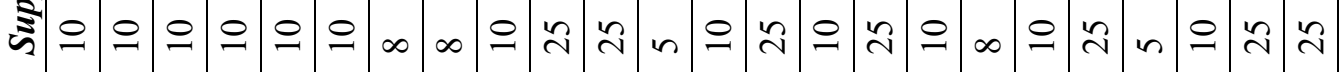

לุ.

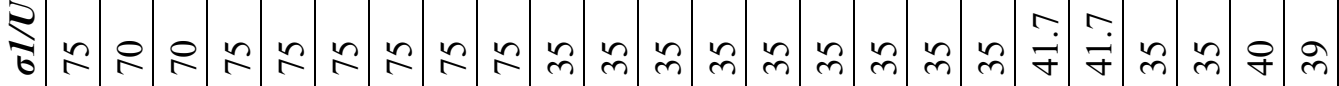

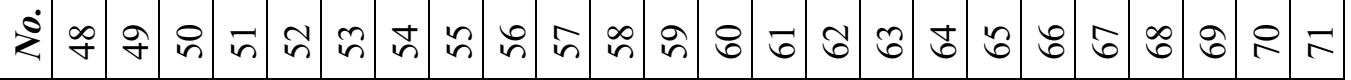




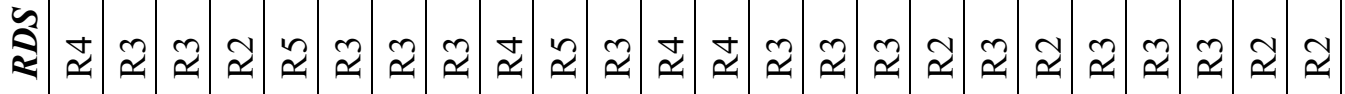

בิ

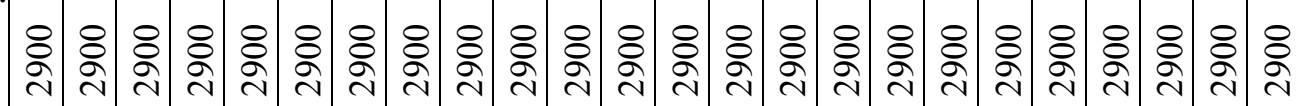

匀

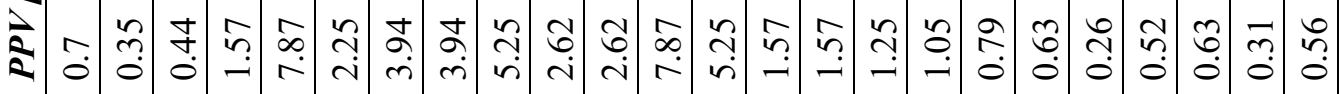

క.

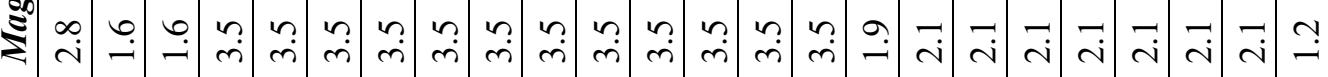

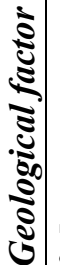

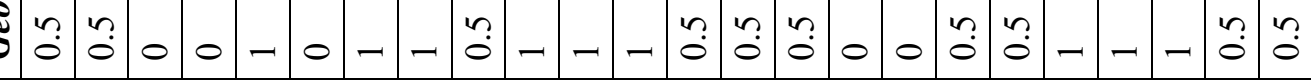

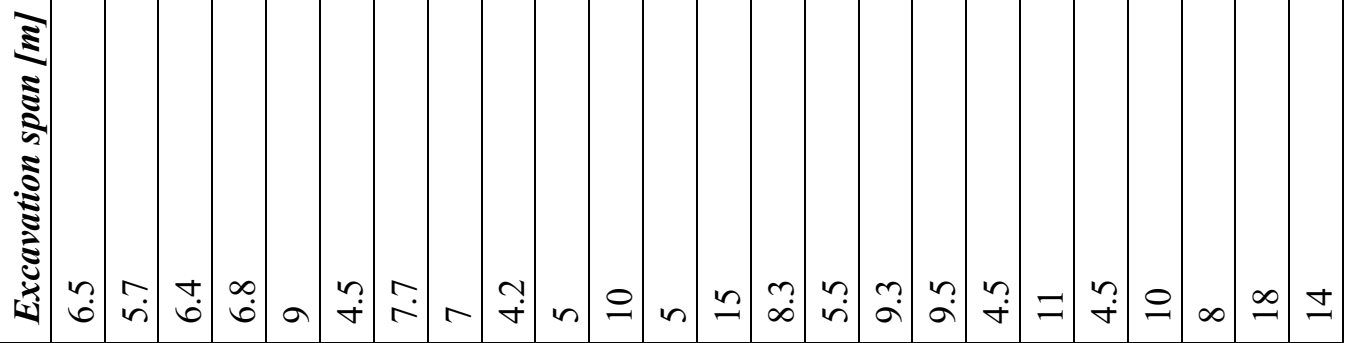

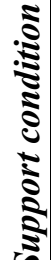

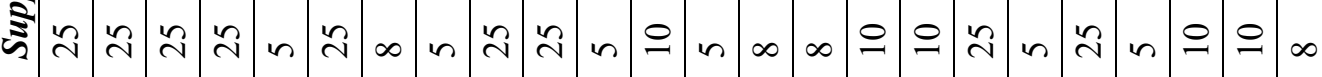

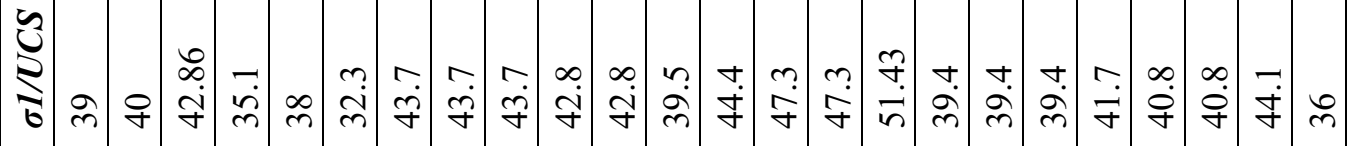

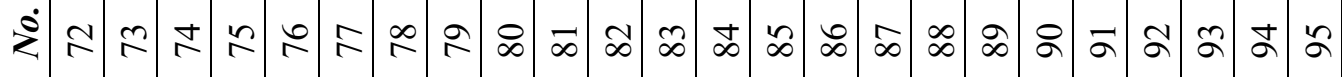




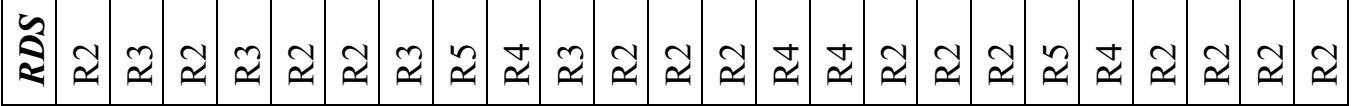

בิ

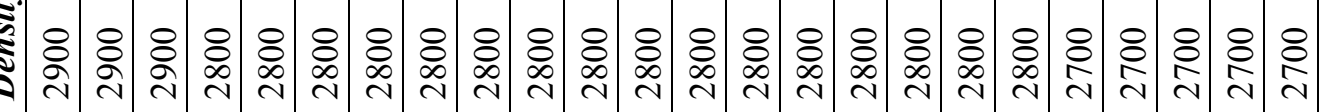

望

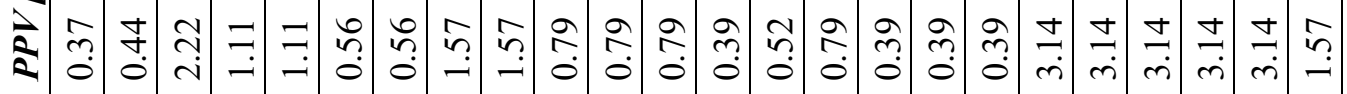

(ะ)

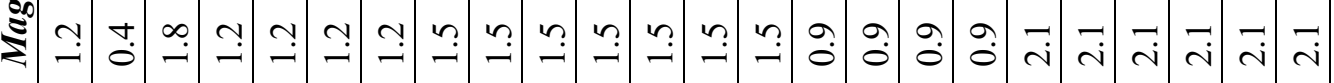

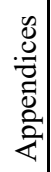
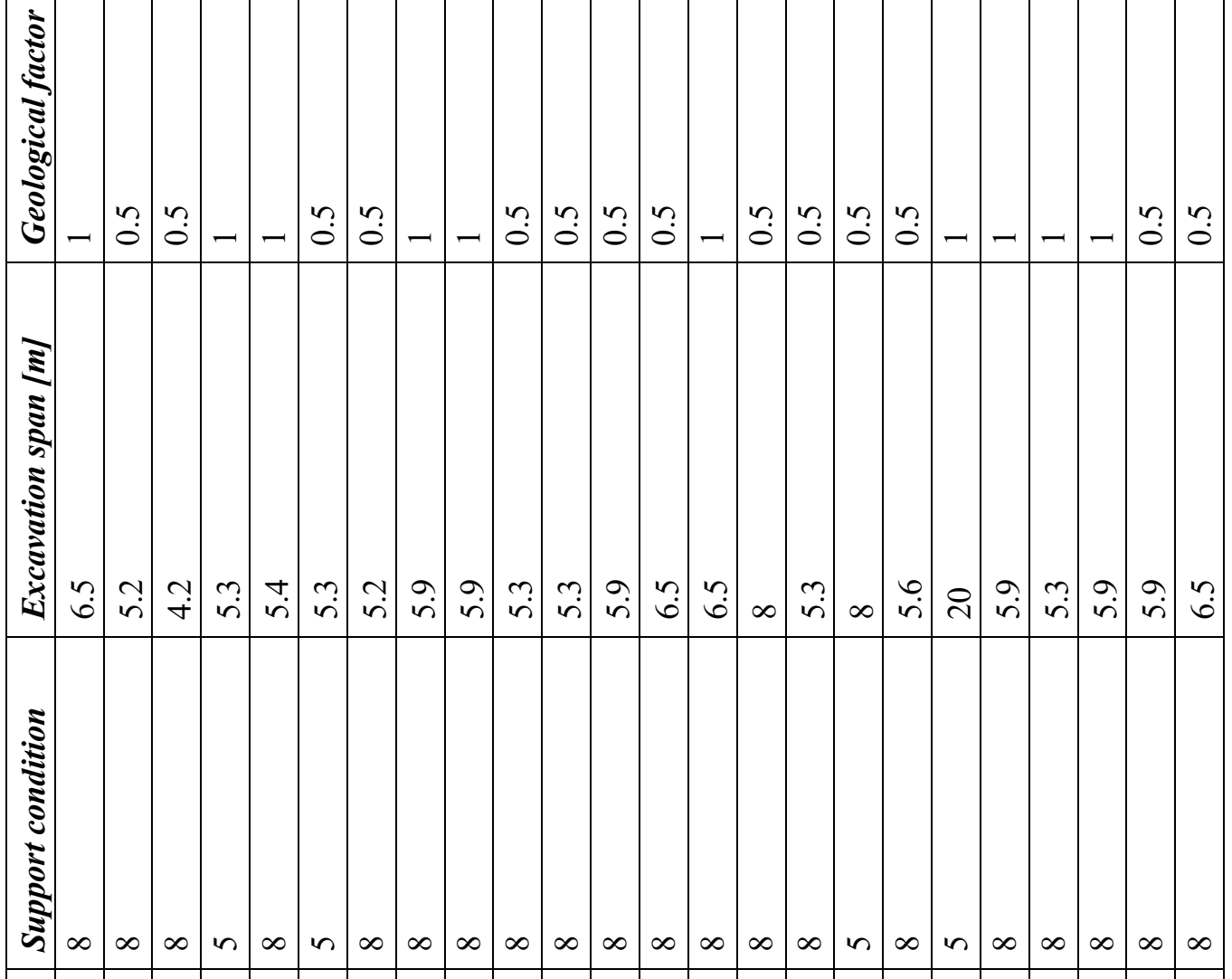

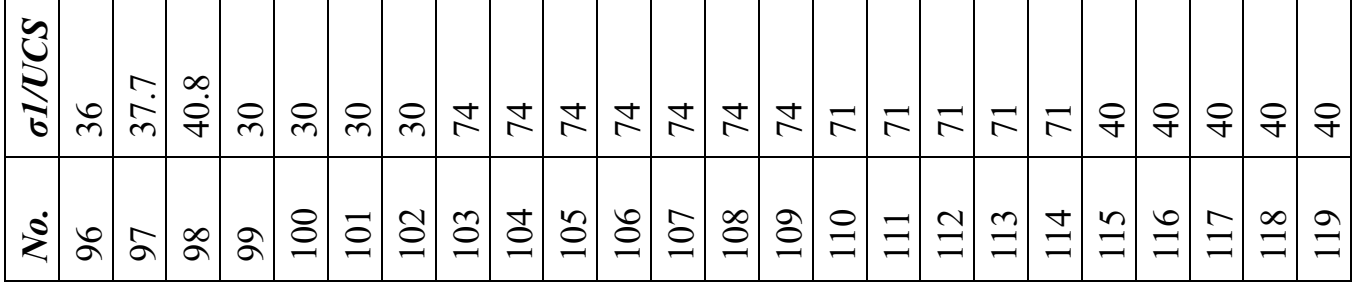




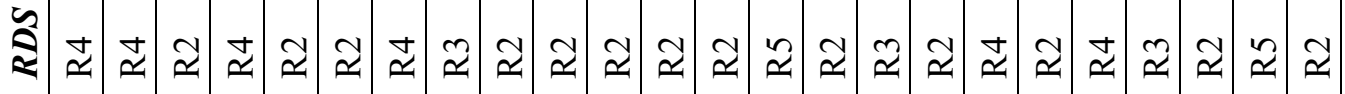

בิ

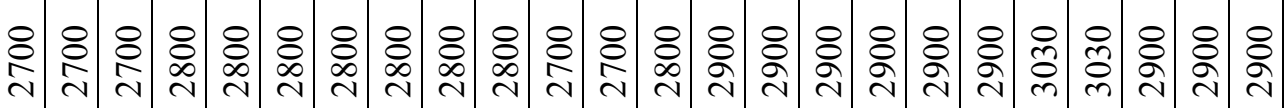

(2)

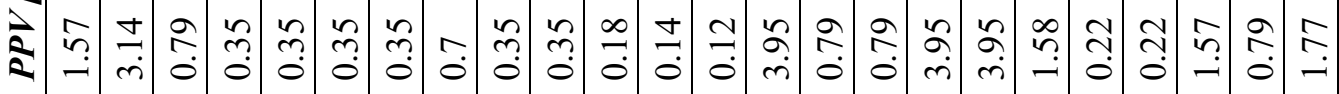

క.

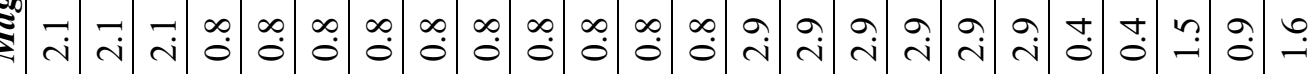

(3)

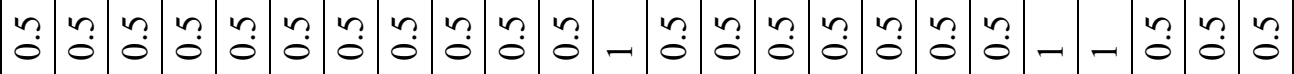

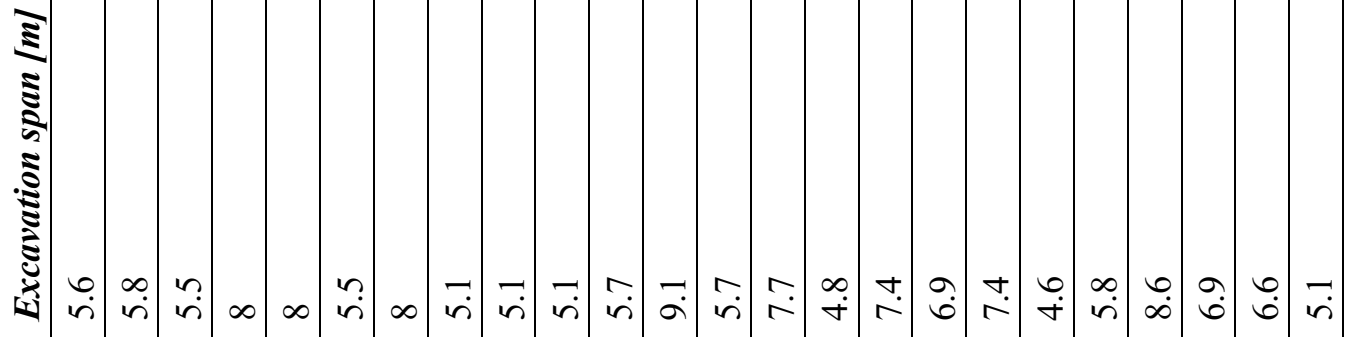

ב⿱艹

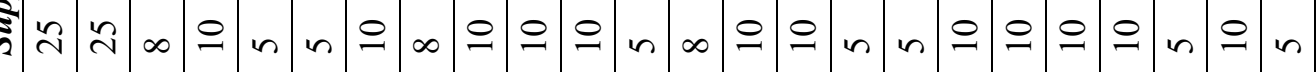

y

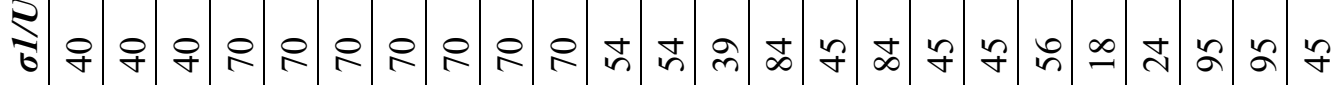

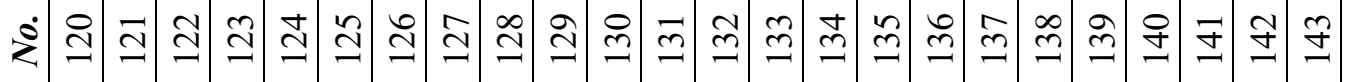




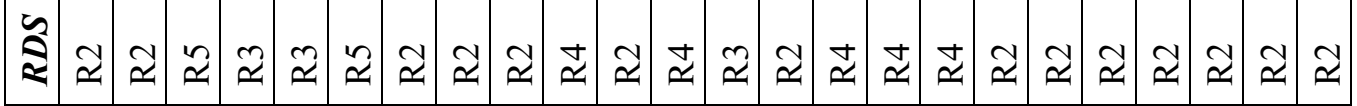

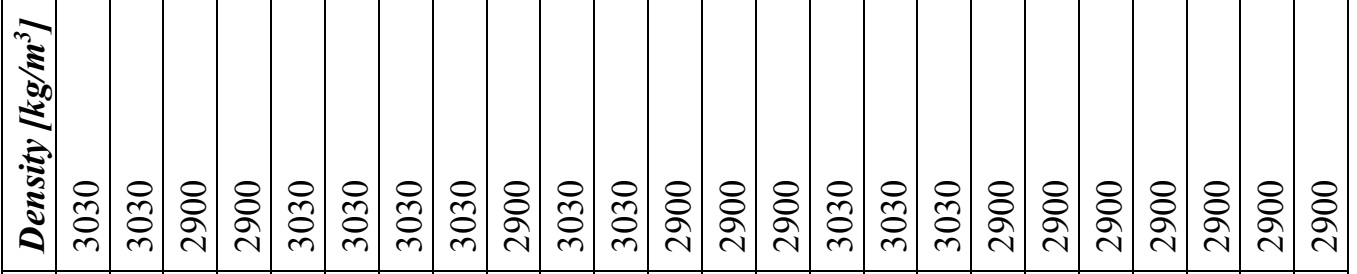

牙

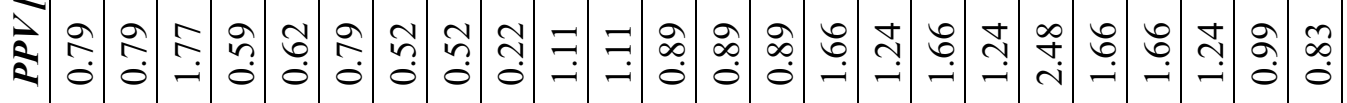

हี้

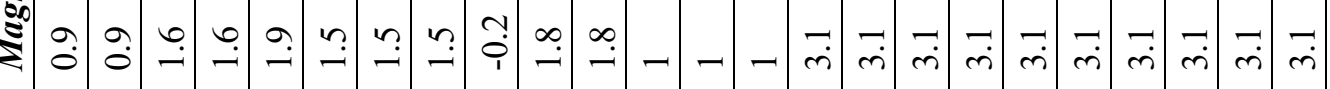
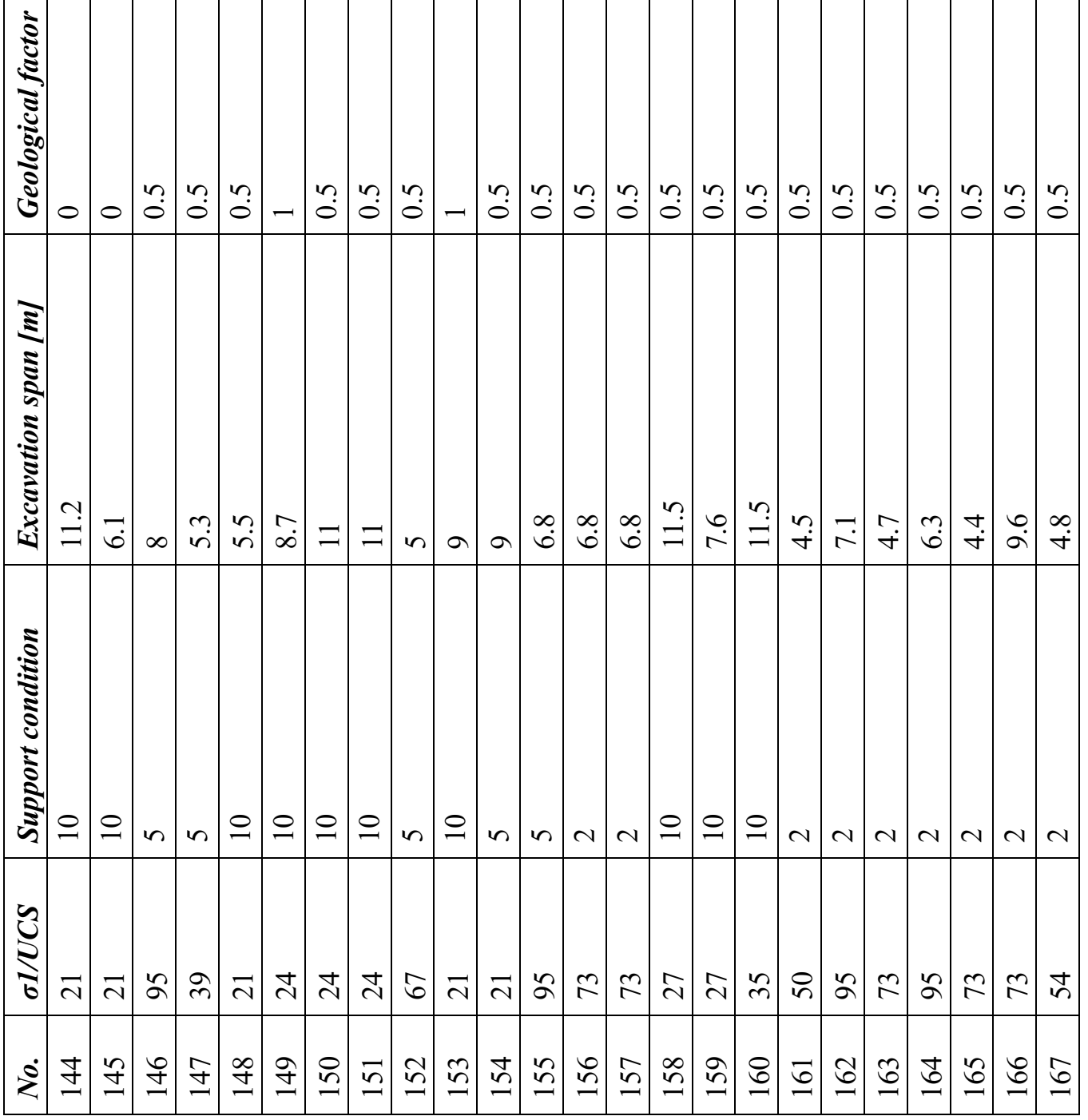


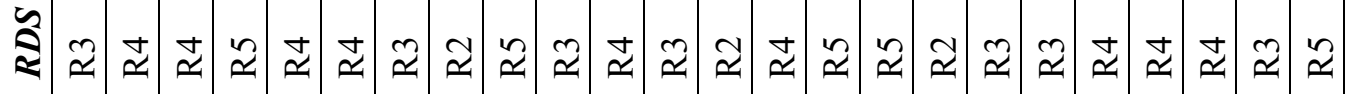

בิ

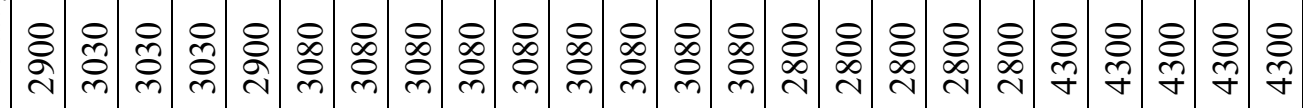

है

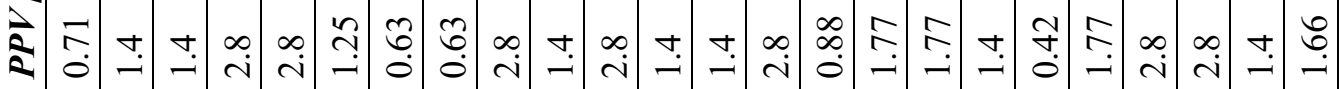

(ะ)

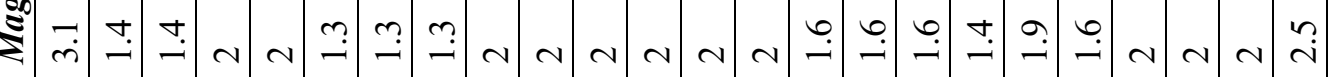

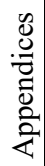
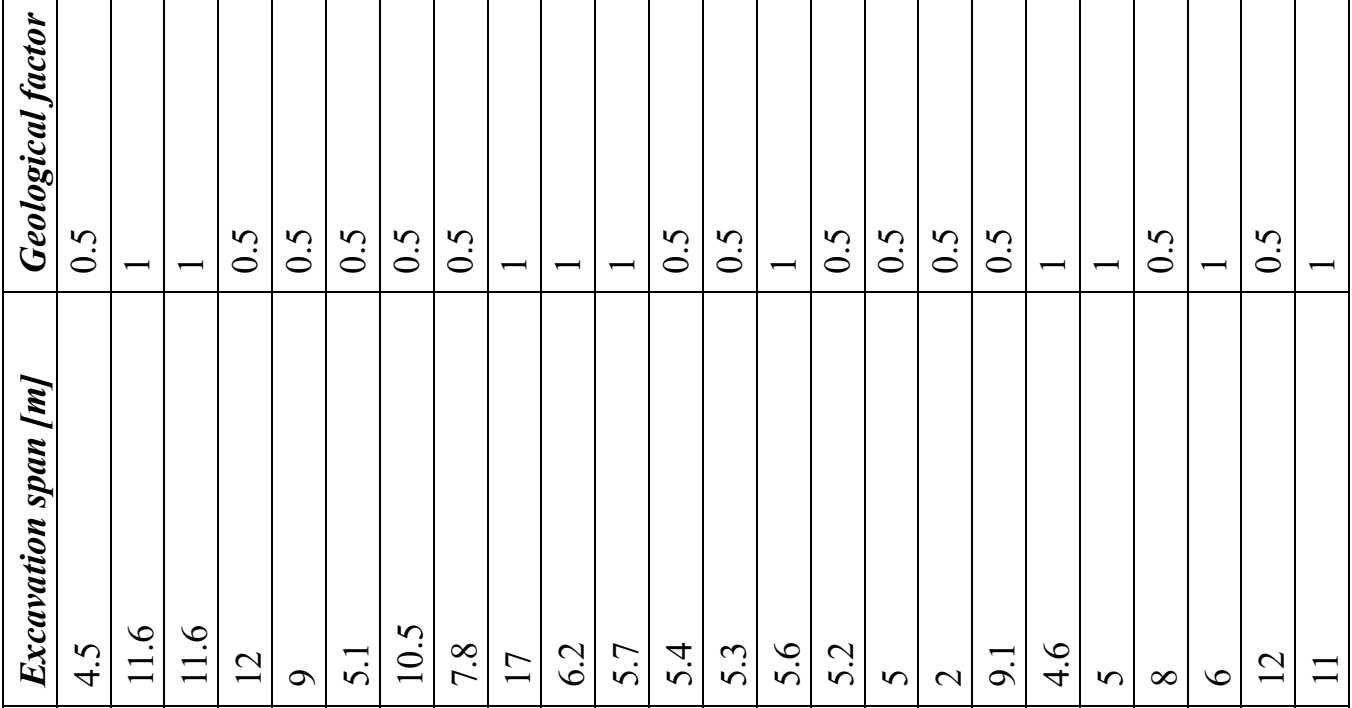

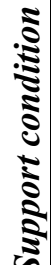

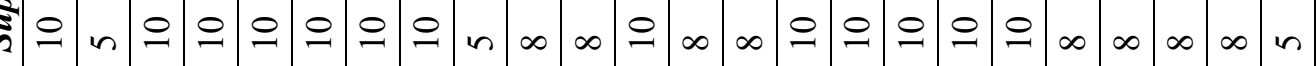

2

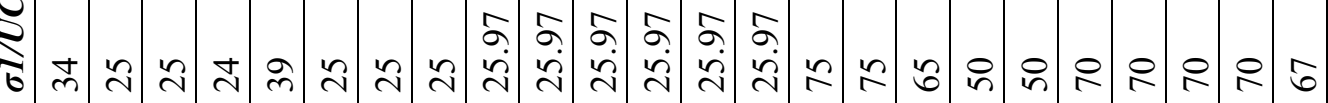

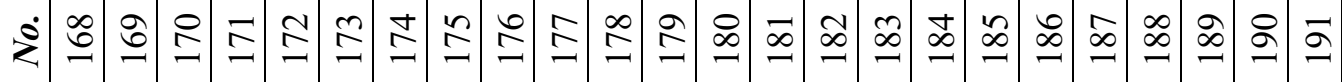




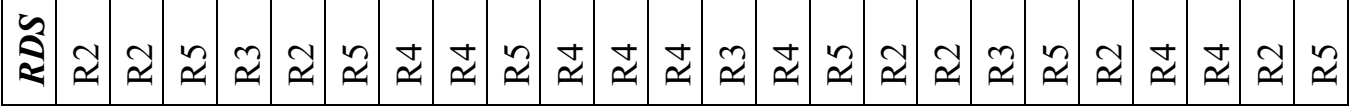

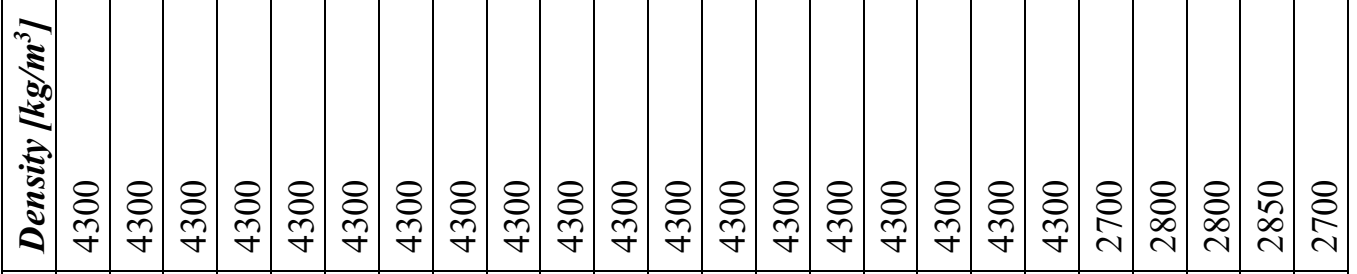

牙

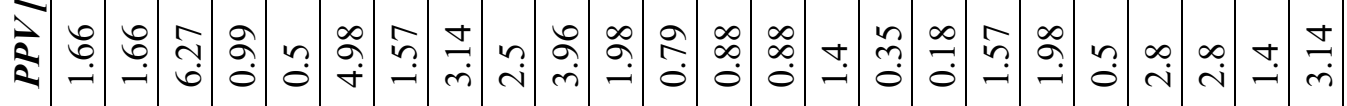

(ะ)

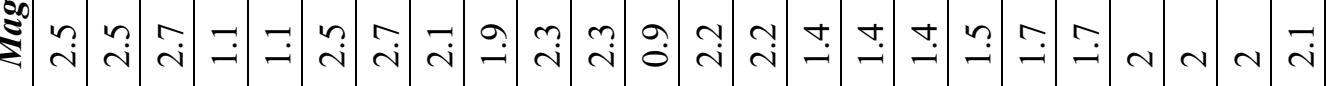
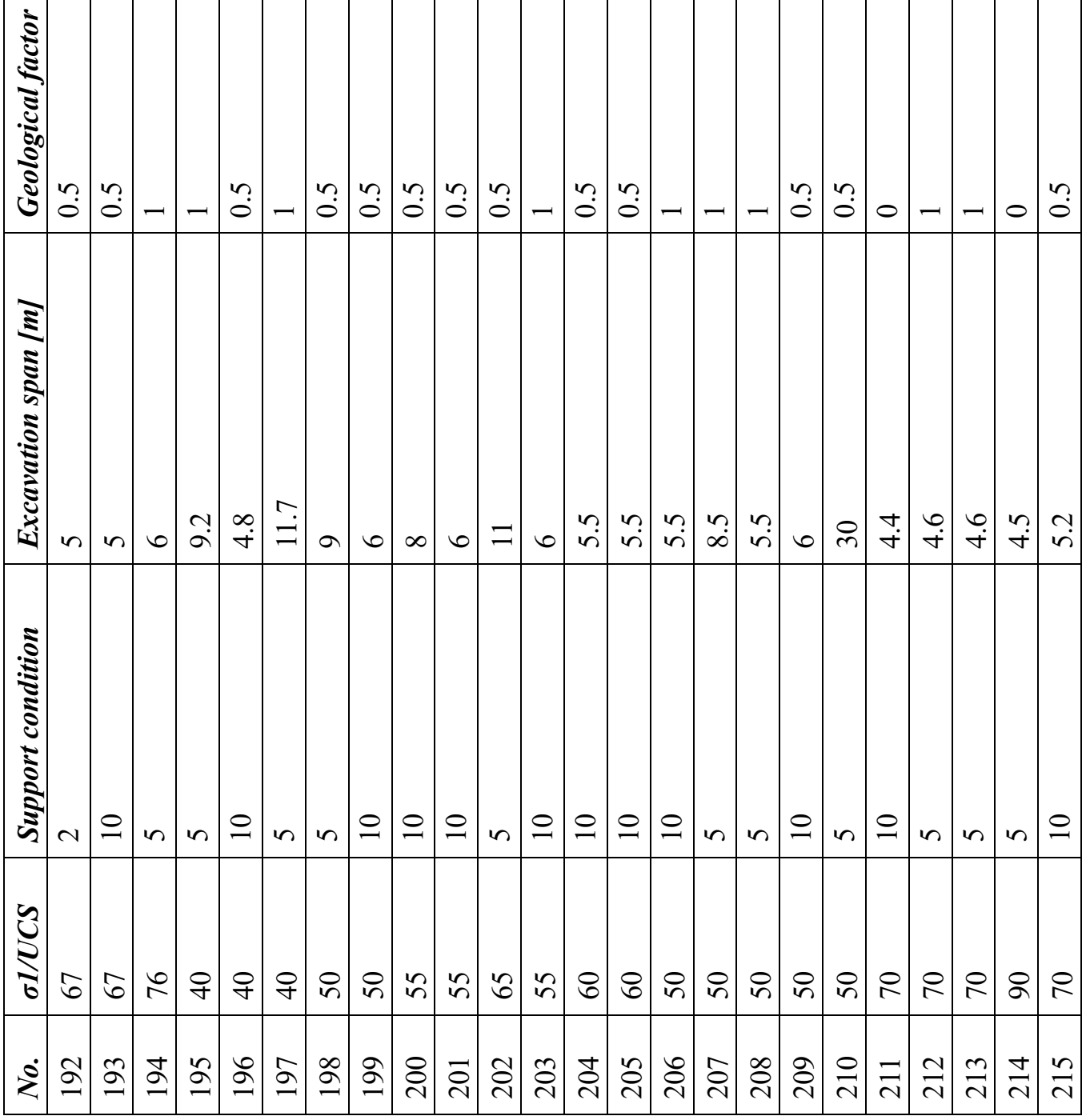


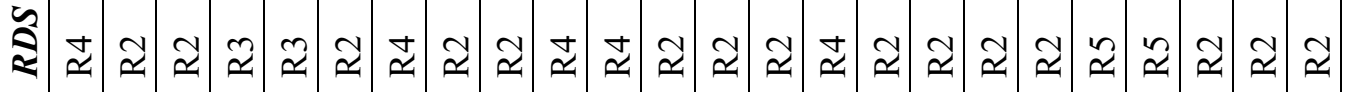

స్이

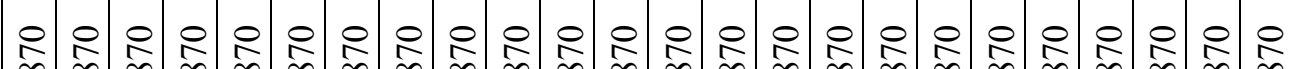

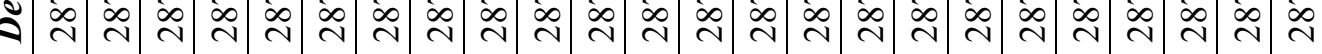

है।

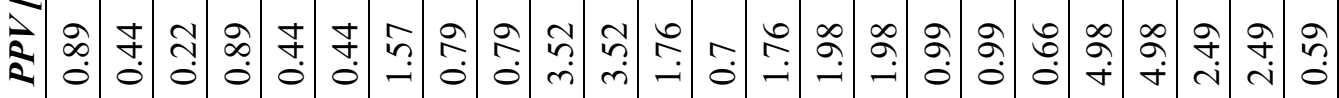

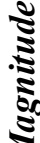

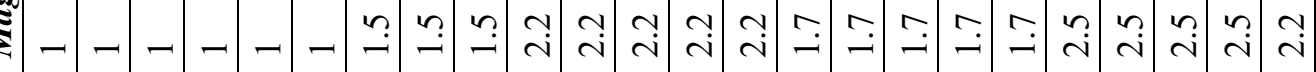

ב⿱艹

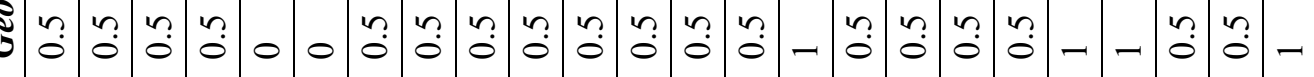

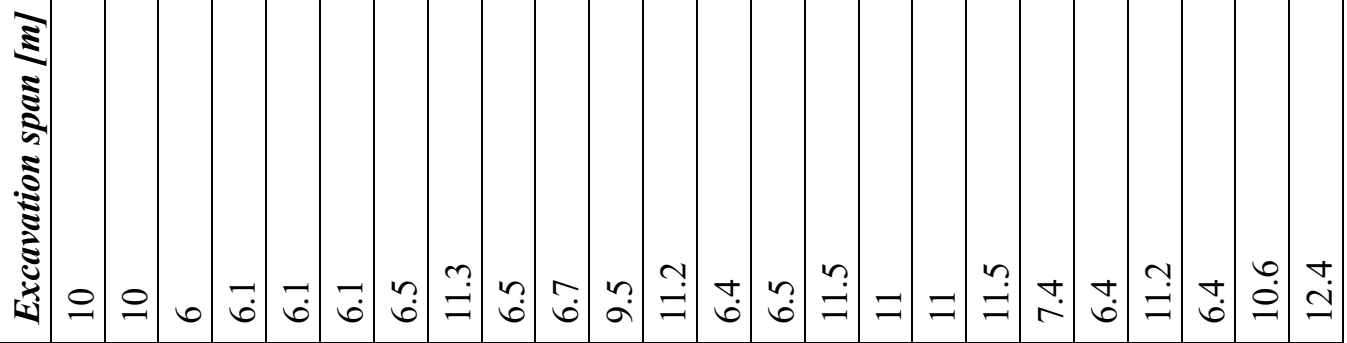

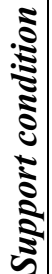

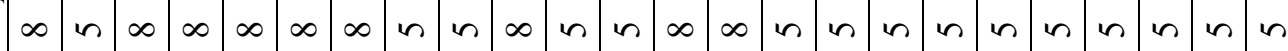

$\widetilde{2}$

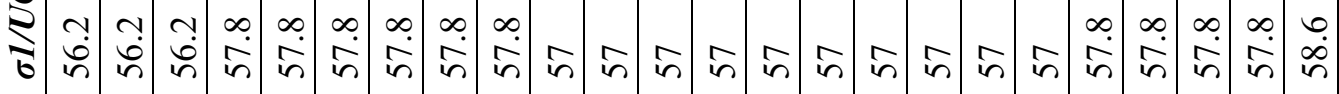

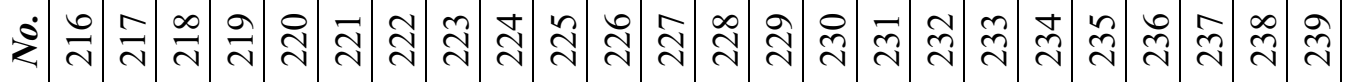




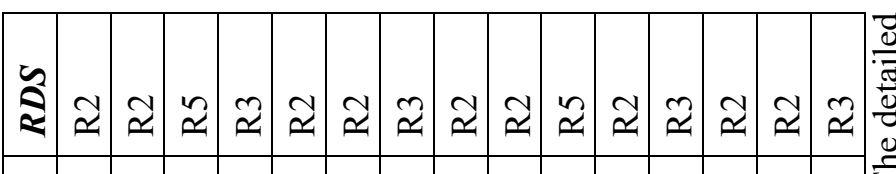

בิ

$\stackrel{80}{=}$

논

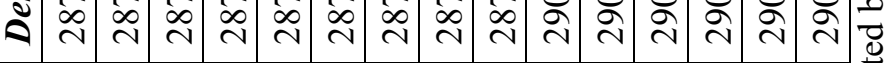

इิ

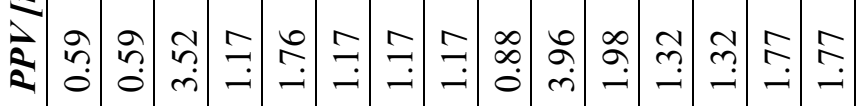

产

焉

离

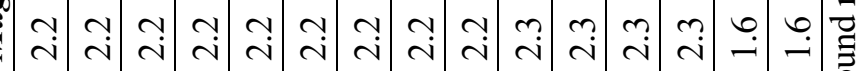

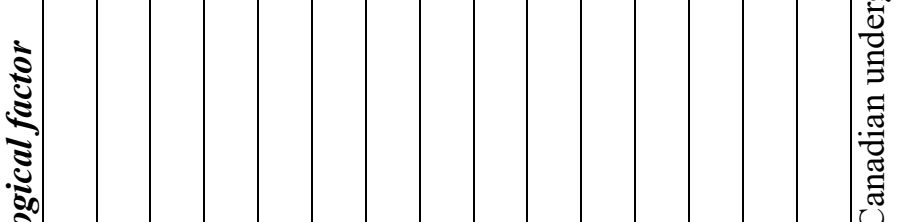

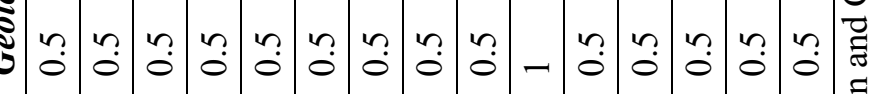

$\gtrsim$

范

능

ミ

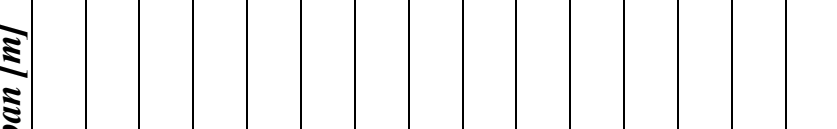

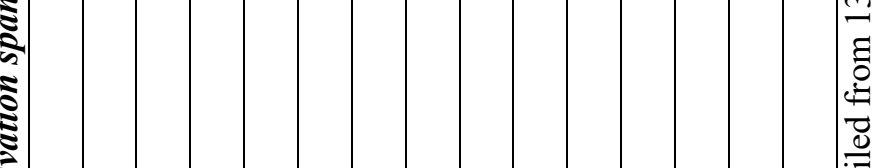

荇

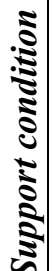

s.

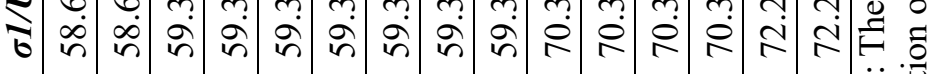

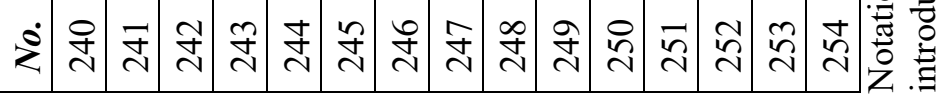


Appendix C Database of 166 case histories of rock tunnel squeezing

\begin{tabular}{|c|c|c|c|c|c|c|}
\hline No. & $H[m]$ & $Q$ & $D[m]$ & $S S R$ & K $[M p a]$ & $\begin{array}{l}\text { Squeezing } \\
\text { condition }\end{array}$ \\
\hline 1 & 150 & 0.4 & 6 & 0.62716 & 26.19048 & $\mathrm{~N}$ \\
\hline 2 & 200 & 0.4 & 6 & 0.335185 & 20 & $\mathrm{~N}$ \\
\hline 3 & 250 & 8.5 & 6 & $*$ & $*$ & $\mathrm{~N}$ \\
\hline 4 & 250 & 8.5 & 14 & $*$ & $*$ & $\mathrm{~N}$ \\
\hline 5 & 350 & 0.5 & 5.8 & $*$ & 2.531646 & $\mathrm{Y}$ \\
\hline 6 & 225 & 3.6 & 5.8 & $*$ & $*$ & $\mathrm{~N}$ \\
\hline 7 & 550 & 4.5 & 5.8 & $*$ & $*$ & $\mathrm{~N}$ \\
\hline 8 & 550 & 3.6 & 5.8 & $*$ & $*$ & $\mathrm{~N}$ \\
\hline 9 & 300 & 0.4 & 5.8 & $*$ & $*$ & $\mathrm{~N}$ \\
\hline 10 & 200 & 0.57 & 7 & $*$ & $*$ & $\mathrm{~N}$ \\
\hline 11 & 175 & 0.84 & 7 & $*$ & $*$ & $\mathrm{~N}$ \\
\hline 12 & 250 & 2.71 & 7 & $*$ & $*$ & $\mathrm{~N}$ \\
\hline 13 & 225 & 3.6 & 4.8 & $*$ & 1000 & $\mathrm{~N}$ \\
\hline 14 & 340 & 1.8 & 4.8 & $*$ & 500 & $\mathrm{~N}$ \\
\hline 15 & 550 & 5.1 & 4.8 & $*$ & 1600 & $\mathrm{~N}$ \\
\hline 16 & 150 & 1.1 & 12 & $*$ & $*$ & $\mathrm{~N}$ \\
\hline 17 & 220 & 0.8 & 12 & $*$ & 32.89474 & $\mathrm{~N}$ \\
\hline 18 & 300 & 6 & 12 & $*$ & $*$ & $\mathrm{~N}$ \\
\hline 19 & 34 & 15 & 13 & $*$ & $*$ & $\mathrm{~N}$ \\
\hline 20 & 52 & 15 & 13 & 2.720798 & 16.66667 & $\mathrm{~N}$ \\
\hline 21 & 280 & 0.05 & 3 & 0.1 & 9.8 & $\mathrm{Y}$ \\
\hline 22 & 280 & 0.022 & 3 & $*$ & 5.96 & $\mathrm{Y}$ \\
\hline 23 & 680 & 0.05 & 9 & $*$ & 9.9 & $\mathrm{Y}$ \\
\hline 24 & 280 & 0.022 & 9 & $*$ & 48.56 & $\mathrm{Y}$ \\
\hline 25 & 100 & 0.0045 & 4.2 & 0.010608 & $*$ & $\mathrm{Y}$ \\
\hline 26 & 112 & 0.006 & 4 & 0.002505 & $*$ & $\mathrm{Y}$ \\
\hline 27 & 111 & 0.008 & 4.3 & 0.020699 & 1936 & $\mathrm{~N}$ \\
\hline 28 & 112 & 0.008 & 4 & 0.002978 & 936 & $\mathrm{~N}$ \\
\hline 29 & 112 & 0.008 & 4 & 0.014888 & 651 & $\mathrm{~N}$ \\
\hline 30 & 140 & 0.009 & 4 & 0.012942 & 430 & $\mathrm{~N}$ \\
\hline 31 & 100 & 0.01 & 4.2 & 0.098241 & $*$ & $\mathrm{Y}$ \\
\hline 32 & 138 & 0.013 & 4 & 0.017959 & 1934 & $\mathrm{~N}$ \\
\hline 33 & 212 & 0.04 & 4.4 & 0.062954 & $*$ & $\mathrm{~N}$ \\
\hline 34 & 300 & 0.05 & 5 & 0.063256 & 1430 & $\mathrm{~N}$ \\
\hline 35 & 112 & 0.06 & 4 & 0.271819 & 458 & $\mathrm{~N}$ \\
\hline 36 & 95 & 0.065 & 4 & 0.295383 & 933 & $\mathrm{~N}$ \\
\hline 37 & 218 & 0.07 & 4 & 0.155149 & 739 & $\mathrm{~N}$ \\
\hline 38 & 98 & 0.08 & 4 & 0.168789 & 933 & $\mathrm{~N}$ \\
\hline 39 & 284 & 0.09 & 5 & 0.238187 & $*$ & $\mathrm{Y}$ \\
\hline 40 & 300 & 0.09 & 5 & 0.088632 & $*$ & $\mathrm{~N}$ \\
\hline 41 & 261 & 0.095 & 4 & 0.259176 & 931 & $\mathrm{~N}$ \\
\hline
\end{tabular}




\begin{tabular}{|c|c|c|c|c|c|c|}
\hline No. & $H[m]$ & $Q$ & $D[m]$ & SSR & K [Mpa] & $\begin{array}{l}\text { Squeezing } \\
\text { condition }\end{array}$ \\
\hline 42 & 198 & 0.14 & 4 & 0.390166 & 934 & $\mathrm{~N}$ \\
\hline 43 & 225 & 0.14 & 4 & 0.090193 & 1430 & $\mathrm{~N}$ \\
\hline 44 & 130 & 0.2 & 5 & 0.468361 & 936 & $\mathrm{~N}$ \\
\hline 45 & 158 & 0.23 & 4.1 & 0.283669 & 650 & $\mathrm{~N}$ \\
\hline 46 & 276 & 0.25 & 5 & 0.331535 & 940 & $\mathrm{~N}$ \\
\hline 47 & 276 & 0.28 & 5 & 0.263853 & 652 & $\mathrm{~N}$ \\
\hline 48 & 126 & 0.3 & 4 & 1.368313 & 461 & $\mathrm{~N}$ \\
\hline 49 & 114 & 0.47 & 4 & 0.756173 & 648 & $\mathrm{~N}$ \\
\hline 50 & 114 & 0.6 & 4 & 0.903542 & 556 & $\mathrm{~N}$ \\
\hline 51 & 300 & 0.023 & 4.6 & 0.101449 & 7.714286 & $\mathrm{Y}$ \\
\hline 52 & 350 & 0.5 & 4.8 & 0.0999 & 25.31646 & $\mathrm{Y}$ \\
\hline 53 & 480 & 0.8 & 2.5 & 0.240385 & $*$ & $\mathrm{Y}$ \\
\hline 54 & 410 & 0.18 & 7 & 0.280348 & $*$ & $\mathrm{Y}$ \\
\hline 55 & 800 & 2.5 & 4.75 & 0.1 & 48.98876 & $\mathrm{Y}$ \\
\hline 56 & 250 & 2.7 & 20 & $*$ & $*$ & $\mathrm{~N}$ \\
\hline 57 & 500 & 0.03 & 9.4 & $*$ & $*$ & $\mathrm{Y}$ \\
\hline 58 & 300 & 1.9 & 9.4 & $*$ & $*$ & $\mathrm{~N}$ \\
\hline 59 & 400 & 0.03 & 9.4 & $*$ & $*$ & $\mathrm{Y}$ \\
\hline 60 & 285 & 0.1 & 7 & $*$ & 9.79 & $\mathrm{Y}$ \\
\hline 61 & 410 & 0.3 & 7 & $*$ & 9.79 & $\mathrm{Y}$ \\
\hline 62 & 415 & 0.88 & 7 & $*$ & 9.79 & $\mathrm{Y}$ \\
\hline 63 & 480 & 0.8 & 2.5 & $*$ & 9.84 & $\mathrm{Y}$ \\
\hline 64 & 500 & 1 & 7 & $*$ & 9.79 & $\mathrm{Y}$ \\
\hline 65 & 510 & 0.88 & 2.5 & $*$ & 9.84 & $\mathrm{Y}$ \\
\hline 66 & 240 & 0.12 & 4.6 & $*$ & 3.97 & $\mathrm{Y}$ \\
\hline 67 & 440 & 0.05 & 4.6 & $*$ & 3.97 & $\mathrm{Y}$ \\
\hline 68 & 450 & 0.06 & 4.6 & $*$ & 3.97 & $\mathrm{Y}$ \\
\hline 69 & 400 & 0.03 & 4.6 & $*$ & 3.98 & $\mathrm{Y}$ \\
\hline 70 & 400 & 0.05 & 4.6 & $*$ & 3.98 & $\mathrm{Y}$ \\
\hline 71 & 200 & 0.02 & 4.6 & $*$ & 2.98 & $\mathrm{Y}$ \\
\hline 72 & 325 & 0.03 & 4.6 & $*$ & 2.98 & $\mathrm{Y}$ \\
\hline 73 & 400 & 0.512 & 4.6 & $*$ & 2.98 & $\mathrm{~N}$ \\
\hline 74 & 700 & 0.3 & 5.8 & $*$ & 9.81 & $\mathrm{Y}$ \\
\hline 75 & 550 & 1.7 & 5.8 & $*$ & 9.81 & $\mathrm{Y}$ \\
\hline 76 & 635 & 4 & 5.8 & $*$ & 9.81 & $\mathrm{Y}$ \\
\hline 77 & 650 & 4.12 & 5.8 & $*$ & 9.81 & $\mathrm{Y}$ \\
\hline 78 & 450 & 0.31 & 5.8 & $*$ & 5.1 & $\mathrm{Y}$ \\
\hline 79 & 750 & 0.5 & 5.8 & $*$ & 8.1 & $\mathrm{Y}$ \\
\hline 80 & 450 & 0.59 & 7 & $*$ & 9.67 & $\mathrm{Y}$ \\
\hline 81 & 337 & 0.007 & 6.8 & $*$ & 44.7619 & $\mathrm{Y}$ \\
\hline 82 & 337 & 0.011 & 6.8 & $*$ & 16.05263 & $\mathrm{Y}$ \\
\hline 83 & 337 & 0.006 & 6.8 & $*$ & 22.58065 & $\mathrm{Y}$ \\
\hline 84 & 337 & 0.006 & 6.8 & $*$ & 36.36364 & $\mathrm{Y}$ \\
\hline
\end{tabular}




\begin{tabular}{|c|c|c|c|c|c|c|}
\hline No. & $H[m]$ & $Q$ & $D[m]$ & SSR & $K[M p a]$ & \begin{tabular}{|l|} 
Squeezing \\
condition
\end{tabular} \\
\hline 85 & 337 & \begin{tabular}{|l|}
0.08 \\
\end{tabular} & 6.8 & $*$ & 14.09091 & $\mathrm{Y}$ \\
\hline 86 & 550 & 0.029 & 8.7 & 0.264879 & 39.13043 & $\mathrm{Y}$ \\
\hline 87 & 600 & 0.023 & 8.7 & 0.242806 & 90.71 & $\mathrm{Y}$ \\
\hline 88 & 600 & 0.03 & 8.7 & 0.242806 & 34.48276 & $\mathrm{Y}$ \\
\hline 89 & 600 & 0.018 & 8.7 & 0.242806 & 26.2 & $\mathrm{Y}$ \\
\hline 90 & 600 & 0.023 & 8.7 & 0.242806 & 28.48 & $\mathrm{Y}$ \\
\hline 91 & 620 & 0.02 & 8.7 & 0.234973 & 26.2 & $\mathrm{Y}$ \\
\hline 92 & 620 & 0.008 & 8.7 & 0.234973 & 14.67 & $\mathrm{Y}$ \\
\hline 93 & 620 & 0.009 & 8.7 & 0.234973 & 14.67 & $\mathrm{Y}$ \\
\hline 94 & 620 & 0.009 & 8.7 & 0.234973 & 14.67 & $\mathrm{Y}$ \\
\hline 95 & 620 & 0.016 & 8.7 & 0.228401 & 26.2 & $\mathrm{Y}$ \\
\hline 96 & 620 & 0.02 & 8.7 & 0.234973 & 26.09756 & $\mathrm{Y}$ \\
\hline 97 & 620 & 0.025 & 8.7 & 0.234973 & 50.8 & $\mathrm{Y}$ \\
\hline 98 & 580 & 0.023 & 8.7 & 0.251178 & 26.2 & $\mathrm{Y}$ \\
\hline 99 & 580 & 0.025 & 8.7 & 0.251178 & 74.66 & $\mathrm{Y}$ \\
\hline 100 & 550 & 0.025 & 8.7 & 0.264879 & 39.87 & $\mathrm{Y}$ \\
\hline 101 & 575 & 0.007 & 8.7 & 0.253363 & 21.16667 & $\mathrm{Y}$ \\
\hline 102 & 700 & 0.417 & 11 & $*$ & 7.428571 & $\mathrm{Y}$ \\
\hline 103 & 700 & 0.333 & 11 & $*$ & 9.142857 & $\mathrm{Y}$ \\
\hline 104 & 750 & 0.333 & 11 & $*$ & 9.142857 & $\mathrm{Y}$ \\
\hline 105 & 600 & 0.25 & 11 & $*$ & 9.142857 & $\mathrm{Y}$ \\
\hline 106 & 850 & 0.056 & 11 & $*$ & 20.4 & $\mathrm{Y}$ \\
\hline 107 & 600 & 0.033 & 11 & $*$ & 33.33333 & $\mathrm{Y}$ \\
\hline 108 & 300 & 0.001 & 11 & $*$ & 16.5 & $\mathrm{Y}$ \\
\hline 109 & 400 & 0.003 & 11 & $*$ & 17 & $\mathrm{Y}$ \\
\hline 110 & 800 & 0.194 & 11 & $*$ & 17.14286 & $\mathrm{Y}$ \\
\hline 111 & 300 & 0.0325 & 6.5 & $*$ & 10 & $\mathrm{Y}$ \\
\hline 112 & 312 & 0.0938 & 6.5 & $*$ & 34.66667 & $\mathrm{Y}$ \\
\hline 113 & 280 & 0.0833 & 6.5 & $*$ & 29.33333 & $\mathrm{Y}$ \\
\hline 114 & 270 & 0.125 & 6.5 & $*$ & 15.90909 & $\mathrm{Y}$ \\
\hline 115 & 285 & 0.0625 & 6.5 & $*$ & 12.8 & $\mathrm{Y}$ \\
\hline 116 & 280 & 0.0313 & 6.5 & $*$ & 11.53846 & $\mathrm{Y}$ \\
\hline 117 & 280 & 0.0417 & 6.5 & $*$ & 12.5 & $\mathrm{Y}$ \\
\hline 118 & 727 & 2.287 & 6 & $*$ & 5.882353 & $\mathrm{Y}$ \\
\hline 119 & 736 & 2.426 & 6 & $*$ & 7.692308 & $\mathrm{Y}$ \\
\hline 120 & 733 & 2.903 & 6 & $*$ & 6.25 & $\mathrm{Y}$ \\
\hline 121 & 690 & 1.65 & 6 & $*$ & 9.375 & $\mathrm{Y}$ \\
\hline 122 & 577 & 1.517 & 13 & $*$ & 11.11111 & $\mathrm{Y}$ \\
\hline 123 & 181.2 & 1.25 & 5.4 & 1.874814 & $*$ & $\mathrm{~N}$ \\
\hline 124 & 199.7 & 0.02 & 5.4 & 0.158038 & 1217.16 & $\mathrm{Y}$ \\
\hline 125 & 217.5 & 0.013 & 5.4 & 0.08152 & 1217.16 & $\mathrm{Y}$ \\
\hline 126 & 252.2 & 0.01 & 5.4 & 0.118383 & 1523.07 & $\mathrm{Y}$ \\
\hline 127 & 246.3 & 0.01 & 5.4 & 0.176716 & 1523.07 & $\mathrm{Y}$ \\
\hline
\end{tabular}




\begin{tabular}{|c|c|c|c|c|c|c|}
\hline No. & $H[m]$ & $Q$ & $D[m]$ & SSR & K [Mpa] & $\begin{array}{l}\text { Squeezing } \\
\text { condition }\end{array}$ \\
\hline 128 & 283.9 & 0.008 & 5.4 & 0.064619 & 1645.38 & $\mathrm{Y}$ \\
\hline 129 & 284.5 & 0.008 & 5.4 & 0.075862 & 1828.98 & $\mathrm{Y}$ \\
\hline 130 & 210.8 & 0.01 & 5.4 & 0.097266 & 1575.72 & $\mathrm{Y}$ \\
\hline 131 & 237.7 & 0.01 & 5.4 & 0.125604 & 1575.72 & $\mathrm{Y}$ \\
\hline 132 & 230 & 0.015 & 5.4 & 0.132937 & 1217.16 & $\mathrm{Y}$ \\
\hline 133 & 222.6 & 0.015 & 5.4 & 0.340967 & 1217.16 & $\mathrm{Y}$ \\
\hline 134 & 600 & $*$ & 5.5 & 0.059952 & $*$ & $\mathrm{Y}$ \\
\hline 135 & 300 & $*$ & 10 & 0.25 & $*$ & $\mathrm{Y}$ \\
\hline 136 & 200 & 0.046416 & 6.5 & 0.330579 & $*$ & $\mathrm{Y}$ \\
\hline 137 & 120 & 0.01 & 16 & 0.089744 & $*$ & $\mathrm{Y}$ \\
\hline 138 & 140 & 0.215443 & 12 & 0.549451 & $*$ & $\mathrm{~N}$ \\
\hline 139 & 80 & 93.5 & 5.4 & 8.610577 & $*$ & $\mathrm{~N}$ \\
\hline 140 & 190 & 7.45 & 5.4 & 1.744939 & $*$ & $\mathrm{~N}$ \\
\hline 141 & 130 & 1.53 & 5.4 & 1.215976 & $*$ & $\mathrm{~N}$ \\
\hline 142 & 300 & 0.215443 & 6.5 & 1.102151 & $*$ & $\mathrm{~N}$ \\
\hline 143 & 300 & 4.641589 & 6.5 & 2 & $*$ & $\mathrm{~N}$ \\
\hline 144 & 110 & 0.046416 & 16 & 0.490076 & $*$ & $\mathrm{~N}$ \\
\hline 145 & 110 & 1 & 16 & 0.869943 & $*$ & $\mathrm{~N}$ \\
\hline 146 & 80 & 10 & $*$ & 1.180173 & $*$ & $\mathrm{~N}$ \\
\hline 147 & 500 & 0.215443 & 6.5 & 0.429936 & $*$ & $\mathrm{Y}$ \\
\hline 148 & 500 & 1 & 6.5 & 0.540105 & $*$ & $\mathrm{Y}$ \\
\hline 149 & 500 & 1 & 6.5 & 1.410256 & $*$ & $\mathrm{~N}$ \\
\hline 150 & 500 & 21.54435 & 6.5 & 2.779553 & $*$ & $\mathrm{~N}$ \\
\hline 151 & 140 & 2.154435 & 12 & 0.860585 & $*$ & $\mathrm{~N}$ \\
\hline 152 & 200 & 0.00631 & 6.5 & 0.14 & $*$ & $\mathrm{Y}$ \\
\hline 153 & 200 & 0.08577 & 6.5 & 0.330579 & $*$ & $\mathrm{Y}$ \\
\hline 154 & 200 & 2.154435 & 6.5 & 0.66 & $*$ & $\mathrm{Y}$ \\
\hline 155 & 400 & 0.001 & 6.5 & 0.07 & $*$ & $\mathrm{Y}$ \\
\hline 156 & 400 & 0.046416 & 6.5 & 0.379939 & $*$ & $\mathrm{Y}$ \\
\hline 157 & 37 & 0.005412 & 11 & 0.263176 & $*$ & $\mathrm{Y}$ \\
\hline 158 & 51 & 0.002929 & 11 & 0.547606 & $*$ & $\mathrm{Y}$ \\
\hline 159 & 60 & 0.008577 & 11 & 0.289865 & $*$ & $\mathrm{Y}$ \\
\hline 160 & 68 & 0.004642 & 11 & 0.143199 & $*$ & $\mathrm{Y}$ \\
\hline 161 & 72 & 0.01 & 11 & 0.241554 & $*$ & $\mathrm{Y}$ \\
\hline 162 & 83 & 0.008577 & 11 & 0.336481 & $*$ & $\mathrm{Y}$ \\
\hline 163 & 84 & 0.008577 & 11 & 0.332475 & $*$ & $\mathrm{Y}$ \\
\hline 164 & 89 & 0.003981 & 11 & 0.10941 & $*$ & $\mathrm{Y}$ \\
\hline 165 & 93 & 0.004642 & 11 & 0.104705 & $*$ & $\mathrm{Y}$ \\
\hline 166 & 94 & 0.007356 & 11 & 0.18502 & $*$ & $\mathrm{Y}$ \\
\hline
\end{tabular}

Notation: The "*” means the unavailable data; "N" indicates case histories with no squeezing, and "Y" indicates squeezing. The case histories of database in this table are compiled by Jimenez and Recio (2011) and extended by Feng and Jimenez (2015). For different Model A, B or C proposed, the sub-sets of the database are selected and employed. 


\section{References of Appendices}

Feng, X., Jimenez, R., 2015. Predicting tunnel squeezing with incomplete data using Bayesian networks. Eng Geol. 195, 214-224.

Guo, D., Zhang, T., Li, Y., et al., 2008. Research on Rockburst Tendency and Its Preventive Measures of $800 \mathrm{~m}$ Deep Surrounding-Rock in Huxi Coal Mine [J]. China Mining Magazine. 17, 50-54.

Heal, D., 2010. Observations and analysis of incidences of rockburst damage in underground mines. University of Western Australia.

Jimenez, R., Recio, D., 2011. A linear classifier for probabilistic prediction of squeezing conditions in Himalayan tunnels. Eng Geol. 121, 101-109.

Zhao, D., Han, C., Yan, H., et al., 2007. The Coal and Rock Physics Mechanics Quality Mensuration and Impact Orientation Estimate of Guantai Coal Mine. Coal. 9, 7.

Zhou, J., Li, X., Shi, X., 2012. Long-term prediction model of rockburst in underground openings using heuristic algorithms and support vector machines. Saf. Sci. 50, 629. 